\title{
Environinental Restoration and Waste Management Program \\ WEST VALLEY TRANSFER CART CONTROL SYSTEM DESIGN DESCRIPTION
}

\author{
E. C. Bradley \\ Robotics \& Process Systems Division \\ R. I. Crutcher \\ J. W. Hálliwell \\ M. S. Hileman \\ M. R. Moore \\ R. N. Nodine \\ F. R. Ruppel \\ R. I. Vandermolen \\ Instrumentation and Controls Division
}

Date Published-January 1993

NOTICE This document contains information of a proliminary nature. It is subject to revision or correction and therefore does not represent a final report.

Prepared by the OAK RIDGE NATIONAL LABORATORY

Oak Ridge, Tennessee 37830 managed by

MARTIN MARIETTA ENERGY SYSTEMS, INC.

for the

U.S. DEPARTMENT OF ENERGY

under contract DE-AC05-84OR21400 


\section{CONTENTS}

LIST OF FIGURES $\ldots \ldots \ldots \ldots \ldots \ldots \ldots \ldots \ldots \ldots \ldots \ldots \ldots \ldots$

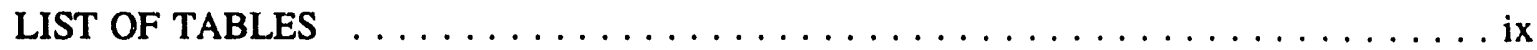

ABSTRACT $\ldots \ldots \ldots \ldots \ldots \ldots \ldots \ldots \ldots \ldots \ldots \ldots \ldots \ldots \ldots \ldots \ldots \ldots \ldots$

ACKNOWLEDGMENTS $\ldots \ldots \ldots \ldots \ldots \ldots \ldots \ldots \ldots \ldots \ldots \ldots \ldots \ldots$

1. INTRODUCTION $\ldots \ldots \ldots \ldots \ldots \ldots \ldots \ldots \ldots \ldots \ldots \ldots \ldots \ldots$

2. DESIGN REQUIREMENTS $\ldots \ldots \ldots \ldots \ldots \ldots \ldots \ldots \ldots \ldots \ldots \ldots \ldots \ldots$

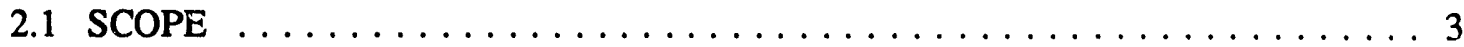

2.2 FUNCTIONAL REQUIREMENTS $\ldots \ldots \ldots \ldots \ldots \ldots \ldots \ldots \ldots$

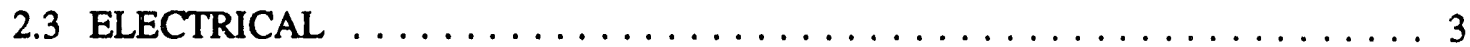

2.4 INSTRUMENTATION AND CONTROLS $\ldots \ldots \ldots \ldots \ldots \ldots \ldots \ldots \ldots$

2.5 OPERATIONAL REQUIREMENTS $\ldots \ldots \ldots \ldots \ldots \ldots \ldots \ldots \ldots \ldots$

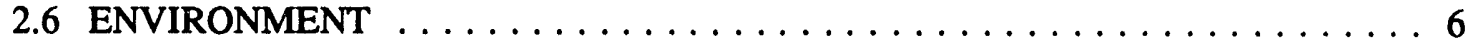

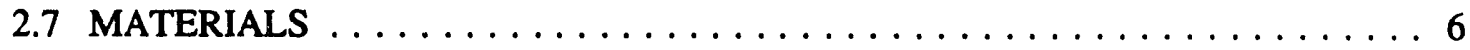

2.8 MAINTENANCE AND REMOTE MAINTENANCE $\ldots \ldots \ldots \ldots \ldots \ldots \ldots 6$

3. SIGNAL TRANSMISSION ASSESSMENT $\ldots \ldots \ldots \ldots \ldots \ldots \ldots \ldots \ldots$

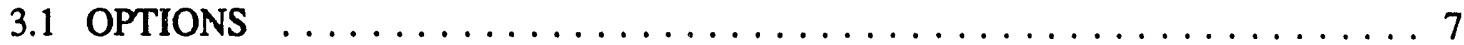

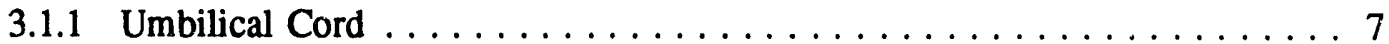

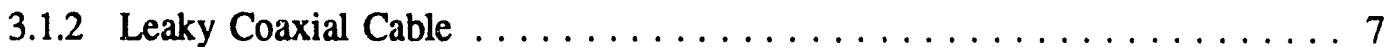

3.1.3 Directive Antenna Link . . . . . . . . . . . . . . . . . . 8

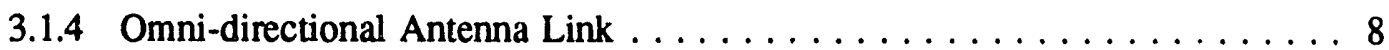

3.2 LITERATURE SURVEY $\ldots \ldots \ldots \ldots \ldots \ldots \ldots \ldots \ldots \ldots \ldots$

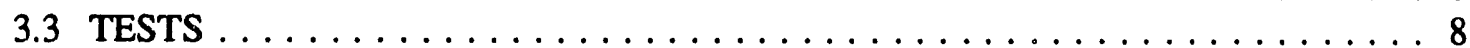

3.3.1 Test Procedure $\ldots \ldots \ldots \ldots \ldots \ldots \ldots \ldots \ldots \ldots \ldots$

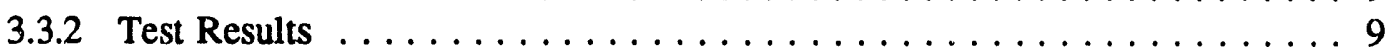

3.4 DESCRIPTION OF PROPOSED SYSTEM $\ldots \ldots \ldots \ldots \ldots \ldots \ldots \ldots$

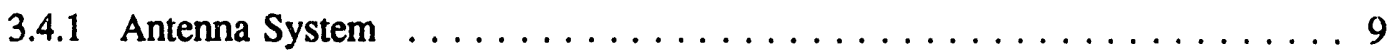

3.4 .2 Communication System $\ldots \ldots \ldots \ldots \ldots \ldots \ldots$

4. SYSTEM OVERVIEW $\ldots \ldots \ldots \ldots \ldots \ldots \ldots \ldots \ldots \ldots \ldots \ldots \ldots$

5. FACILITY SYSTEMS $\ldots \ldots \ldots \ldots \ldots \ldots \ldots \ldots \ldots \ldots \ldots \ldots \ldots \ldots \ldots \ldots$

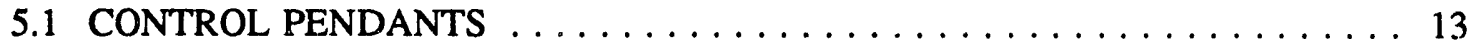

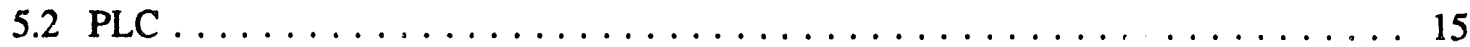


5.3 ENGINEER'S CONSOLE COMPUTER $\ldots \ldots \ldots \ldots \ldots \ldots \ldots \ldots \ldots$

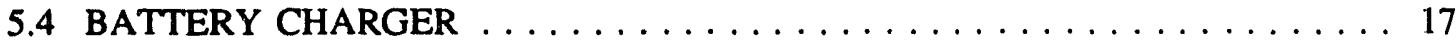

5.5 BATTERY CHARGER INTERFACE CIRCUIT $\ldots \ldots \ldots \ldots \ldots \ldots \ldots \ldots$

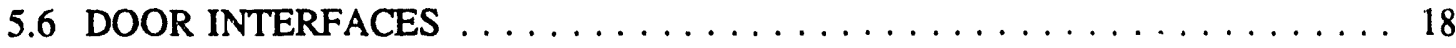

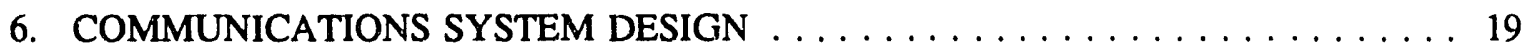

6.1 FACILITY TRANSMITTER CIRCUIT $\ldots \ldots \ldots \ldots \ldots \ldots \ldots \ldots \ldots$

6.2 FACILITY TRANSMIT/RECEIVE CIRCUIT $\ldots \ldots \ldots \ldots \ldots \ldots \ldots \ldots$

6.3 FACILITY ANTENNA SWITCH CIRCUIT $\ldots \ldots \ldots \ldots \ldots \ldots \ldots \ldots$

6.4 FACILITY AND CART RECEIVER CIRCUITS $\ldots \ldots \ldots \ldots \ldots \ldots \ldots$

6.5 CART ANTENNA CONTROL CIRCUIT $\ldots \ldots \ldots \ldots \ldots \ldots \ldots \ldots \ldots$

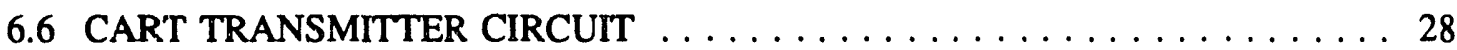

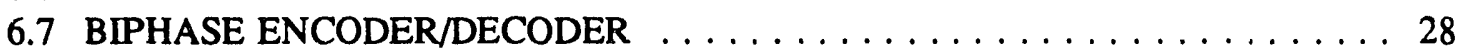

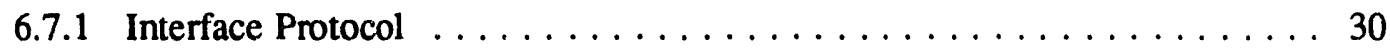

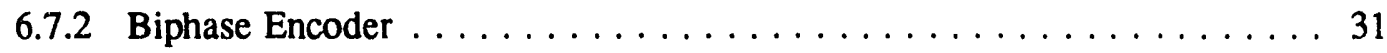

6.7.3 Biphase Decoder . . . . . . . . . . . . . . . . . 35

6.8 FACILITY COMMUNICATIONS SYSTEM CONTROLLER $\ldots \ldots \ldots \ldots \ldots . . \ldots 4$

6.9 FACILITY ANTENNAS $\ldots \ldots \ldots \ldots \ldots \ldots \ldots \ldots \ldots \ldots$

7. CART ELECTRONICS SYSTEM $\ldots \ldots \ldots \ldots \ldots \ldots \ldots \ldots \ldots \ldots \ldots \ldots$

7.1 SYSTEM CONTROLLER $\ldots \ldots \ldots \ldots \ldots \ldots \ldots \ldots \ldots \ldots \ldots \ldots$

7.1.1 Interface to Cart Communications System . . . . . . . . . . . . 47

7.1 .2 Interface to Motor Drives . . . . . . . . . . . . . . . . 47

7.1.3 Interface to Analog-to-Digital Converter . . . . . . . . . . . 48

7.1.4 Interface to Cart Interface Module . . . . . . . . . . . . . . . . 49

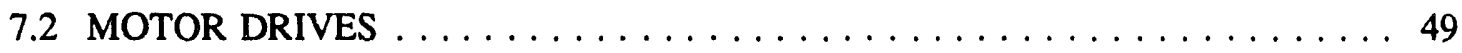

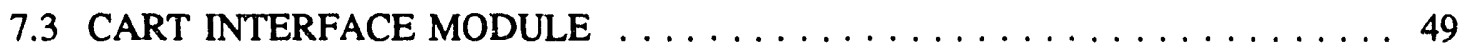

7.3.1 Battery Switching During Charging . . . . . . . . . . . . . . 49

7.3.2 Current Shunt and Voltage Buffers $\ldots \ldots \ldots \ldots \ldots \ldots \ldots \ldots$

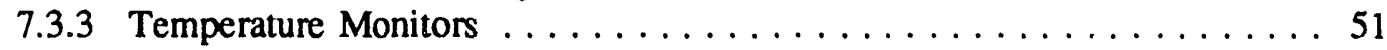

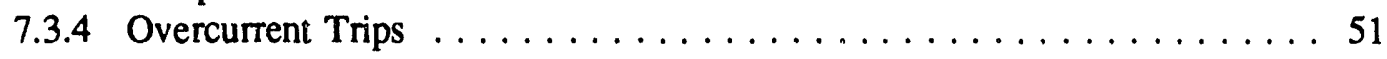

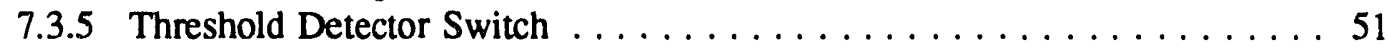

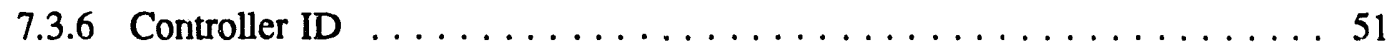

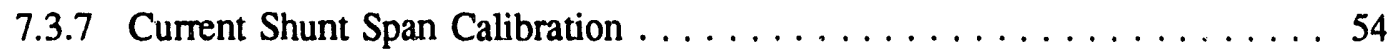

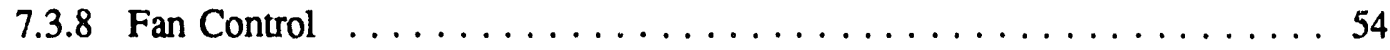

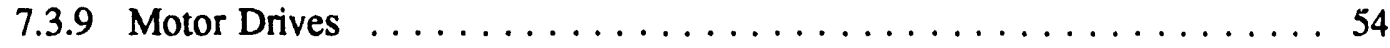

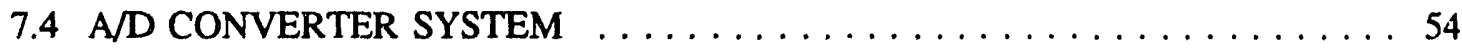

7.5 DOOR THRESHOLD LIMIT SWITCH $\ldots \ldots \ldots \ldots \ldots \ldots \ldots \ldots \ldots$

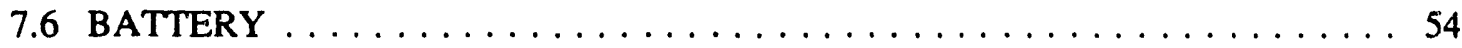

8. CONTROL MODULE ENCLOSURE ASSEMBLY $\ldots \ldots \ldots \ldots \ldots \ldots \ldots \ldots$

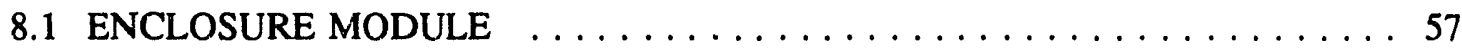

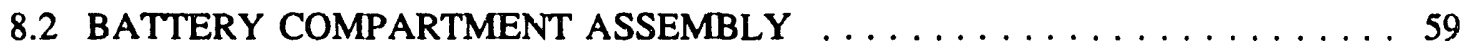

8.3 FRONT COVER AND THRESHOLD LIMIT SWITCH $\ldots \ldots \ldots \ldots \ldots \ldots \ldots$

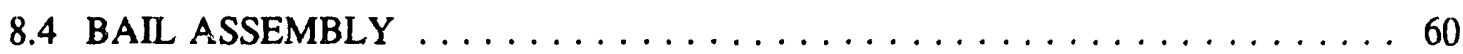

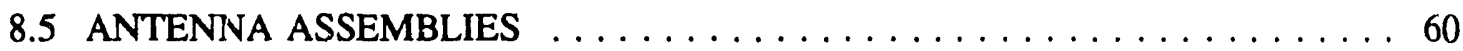

8.6 SHIELDED ELECTRONICS ENCLOSURE $\ldots \ldots \ldots \ldots \ldots \ldots \ldots \ldots \ldots$

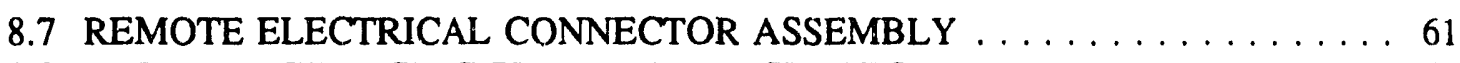

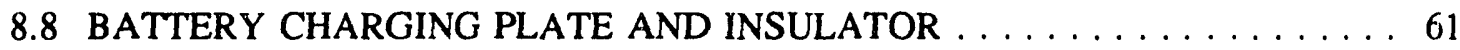




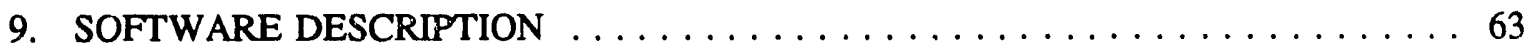

9.1 ENGINEER'S CONSOLE COMPUTER INTERFACE SOFTWARE $\ldots \ldots \ldots 63$

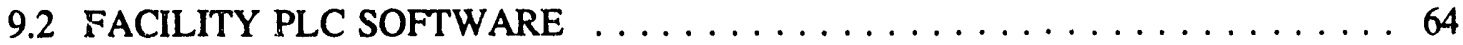

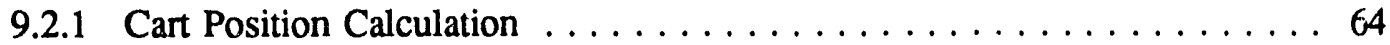

9.2.2 Battery State of Charge . . . . . . . . . . . . . . . . . 66

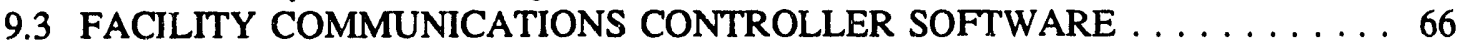

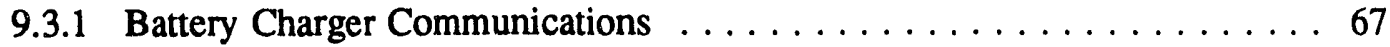

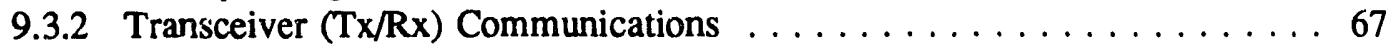

9.4 CART CONTROLLER SOFTWARE $\ldots \ldots \ldots \ldots \ldots \ldots \ldots \ldots \ldots \ldots$

9.4.1 Data Packet Status . . . . . . . . . . . . . . . . . . . . . . . 67

9.5 COMMUNICATIONS PROTOCOL AND COMMAND DEFINITIONS . . . . . 68

9.5.1 Reasons for Using Communications Protocol . . . . . . . . . . . . . . 69

9.5.2 Description of Communications Protocol . . . . . . . . . . . . . 69

9.5.3 Exception to Communications Protocol ............... 71

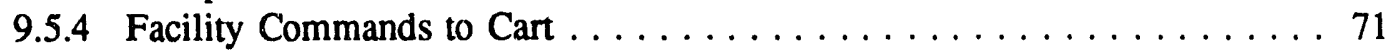

9.5.5 Cart System Status Report $\ldots \ldots \ldots \ldots \ldots \ldots \ldots \ldots \ldots$

10. SUMMARY AND CONSIDERATIONS $\ldots \ldots \ldots \ldots \ldots \ldots \ldots \ldots$

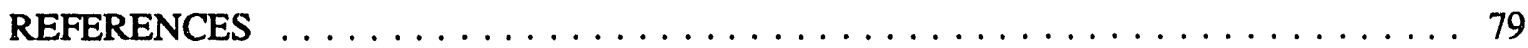

\section{APPENDIXES}

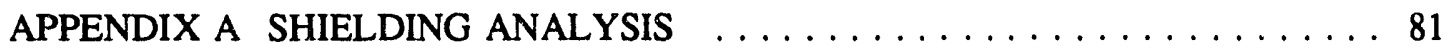

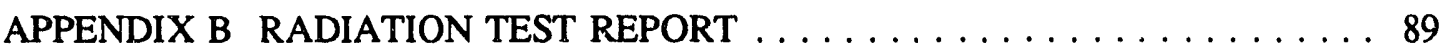

APPENDIX C BATTERY EVALUATION AND TEST REPORT $\ldots \ldots \ldots \ldots \ldots 95$

APPENDIX D PROTOTYPE HARDWARE TESTING $\ldots \ldots \ldots \ldots \ldots \ldots \ldots \ldots 121$

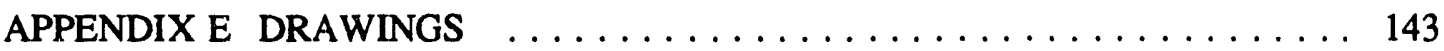

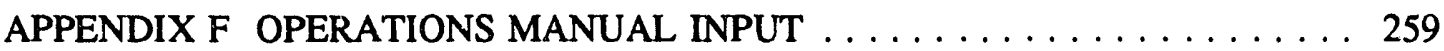




\section{LIST OF FIGURES}

Fig. 2.1. West Valley Demonstration Project transfer cart cell plan . . . . . . . . . 5

Fig. 3.1. Mounting of dipole antennas on cart $\ldots \ldots \ldots \ldots \ldots \ldots \ldots \ldots \ldots$

Fig. 4.1. Overview of transfer cart control system $\ldots \ldots \ldots \ldots \ldots \ldots \ldots \ldots \ldots$

Fig. 5.1. Operator's control pendant $\ldots \ldots \ldots \ldots \ldots \ldots \ldots \ldots \ldots \ldots \ldots \ldots \ldots$

Fig. 5.2. Engineer's console $\ldots \ldots \ldots \ldots \ldots \ldots \ldots \ldots \ldots \ldots \ldots \ldots \ldots \ldots \ldots \ldots 16$

Fig. 5.3. Charger polarity control switching $\ldots \ldots \ldots \ldots \ldots \ldots \ldots \ldots \ldots \ldots \ldots$

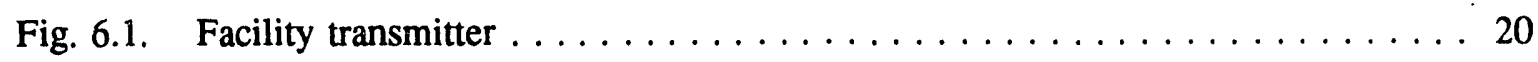

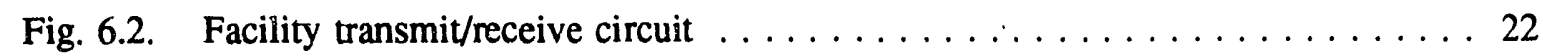

Fig. 6.3. Facility antenna switch $\ldots \ldots \ldots \ldots \ldots \ldots \ldots \ldots \ldots \ldots \ldots \ldots \ldots$

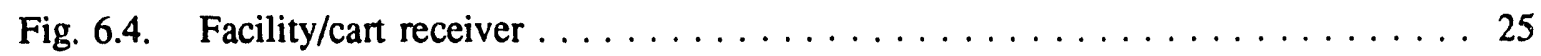

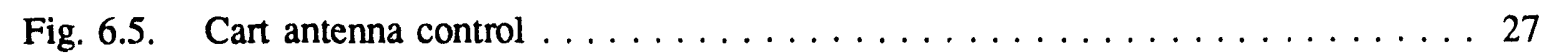

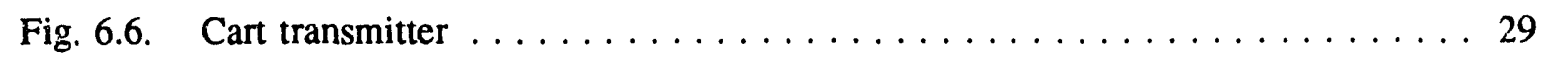

Fig. 6.7. Biphase encoder/CRC generator timing diagram with data values $\ldots \ldots \ldots 33$

Fig. 6.8. Biphase encoder/CRC checker normal transmission timing diagram . . . . . 37

Fig. 6.9. Biphase decoder/CRC checker reset and error timing diagram . . . . . . . 41

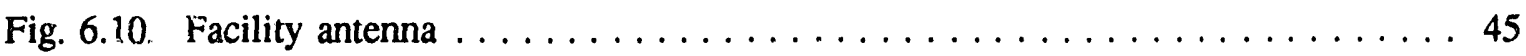

Fig. 7.1. Cart electronics rack card arrangement $\ldots \ldots \ldots \ldots \ldots \ldots \ldots \ldots$

Fig. 7.2. Cart controller interface module block diagram $\ldots \ldots \ldots \ldots \ldots \ldots \ldots$

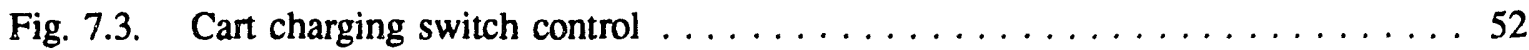

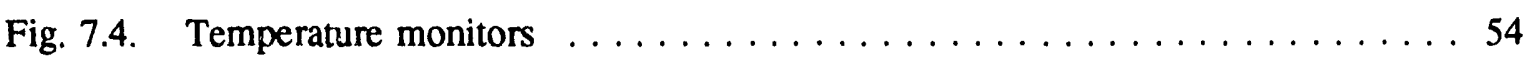

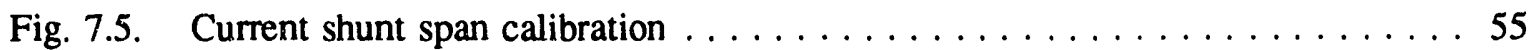

Fig. 8.1. Two views of the control module enclosure assembly $\ldots \ldots \ldots \ldots \ldots$

Fig. 9.1. Cart distance definitions for position algorithm $\ldots \ldots \ldots \ldots \ldots \ldots$

Fig. A.1. Proposed shielding design for the West Valley Vitrification Facility cart . . . 82

Fig. A.2. Characteristics of the West Valley Nuclear Services canisters . . . . . . . . 84

Fig. A.3. Gamma-ray isodose rate contours ( $\mathrm{rad}-\mathrm{Si} / \mathrm{hr}$ ) for an elevation view model of the West Valley Vitrification Facility cart (Sect. B-B of Fig. A.1) . . . . . . . 87

Fig. A.4. Gamma-ray isodose rate contours ( $\mathrm{rad}-\mathrm{Si} / \mathrm{hr})$ for a plan view model of the West

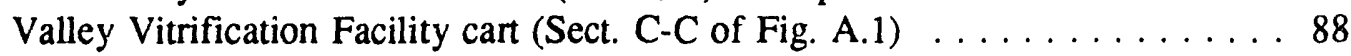

Fig. B.1. Equipment arrangement for radiation test $\ldots \ldots \ldots \ldots \ldots \ldots \ldots \ldots$

Fig. C.1. Battery discharge current profile (idle current $=1.75 \mathrm{~A}$ ) $\ldots \ldots \ldots \ldots$

Fig. C.2. Battery discharge current profile (idle current $=3.0 \mathrm{~A}) \ldots \ldots \ldots$ 
Fig. C.3. Discharge test configuration $\ldots \ldots \ldots \ldots \ldots \ldots \ldots \ldots \ldots \ldots \ldots$

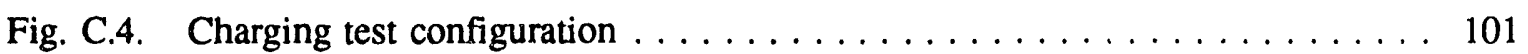

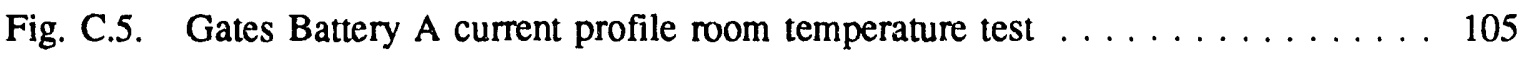

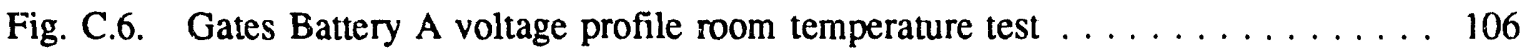

Fig. C.7. Powersonic Battery A current profile room temperature test . . . . . . . . 107

Fig. C.8. Powersonic Battery A voltage profile room temperature test . . . . . . . . 108

Fig. C.9. Gates Battery A current profile $20^{\circ} \mathrm{F}$ test $\ldots \ldots \ldots \ldots \ldots \ldots \ldots$

Fig. C.10. Gates Battery A voltage profile $20^{\circ} \mathrm{F}$ test $\ldots \ldots \ldots \ldots \ldots \ldots \ldots \ldots$

Fig. C.11. Gates Battery B current profile $20^{\circ} \mathrm{F}$ test $\ldots \ldots \ldots \ldots \ldots \ldots \ldots \ldots$

Fig. C.12. Gates Battery B voltage profile $20^{\circ} \mathrm{F}$ test $\ldots \ldots \ldots \ldots \ldots \ldots \ldots \ldots \ldots \ldots$

Fig. C.13. Powersonic Battery A current profile $20^{\circ} \mathrm{F}$ test $\ldots \ldots \ldots \ldots \ldots \ldots \ldots 113$

Fig. C.14. Powersonic Battery A voltage profile $20^{\circ} \mathrm{F}$ test $\ldots \ldots \ldots \ldots \ldots \ldots \ldots 114$

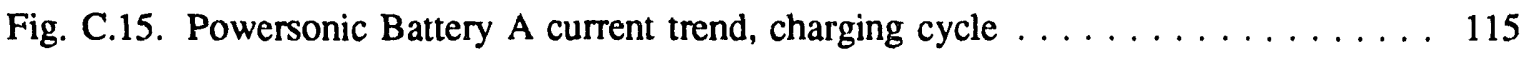

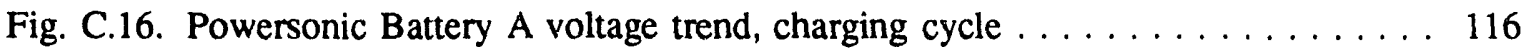

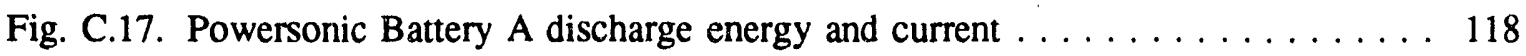

Fig. C.18. Powersonic Battery A energy retumed and current during battery recharging vs elapsed time . . . . . . . . . . . . . . . . . . . . . . . . . 119

Fig. D.1. Equipment arrangement for integrated system testing $\ldots \ldots \ldots \ldots \ldots \ldots 123$ 


\section{LIST OF TABLES}

Table 7.1. Summary of cart energy requirements for 1 cycle based cri prototype testing . . 56

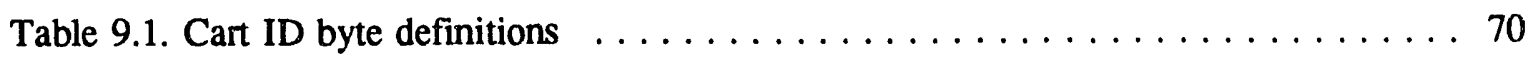

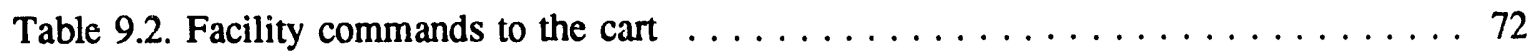

Table 9.3. Cart system status report $\ldots \ldots \ldots \ldots \ldots \ldots \ldots \ldots \ldots \ldots \ldots \ldots$

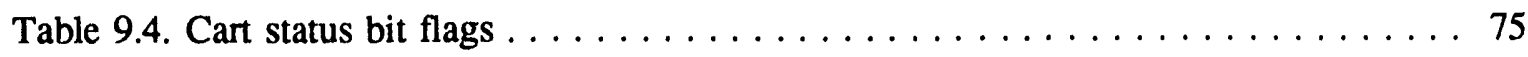




\begin{abstract}
Detail design of the control system for the West Valley Nuclear Services Vitrification Facility transfer cart has been completed by Oak Ridge National Laboratory. This report documents the requirements and describes the detail design of that equipment and control software. Copies of significant design documents including analysis and testing reports and design drawings are included in the Appendixes.
\end{abstract}




\section{ACKNOWLEDGMENTS}

The authors gratefully acknowledge the contributions of the following people whose efforts are also exhibited in this report. Allen Blalock was the lead engineer for cart control system electronics design and performed radiation testing and prototype hardware testing. Jerry Stapleton was the lead engineer for the control module enclosure and facility antenna mechanical design. Charles Slater performed the shielding analysis for the control module enclosure. The designers responsible for preparing the drawing package include Bob Maples, Karen Cutshaw, Mike Cutshaw, Elaine Cooper, Saundra Shelton, Shirley Shugart, Gene Keith, Doug Bailes, Kevin Farley, Michelle Hollis, and Don Rainey. In addition, Rick Meigs and John Victor, West Valley Nuclear Services, cooperated throughout the effort by providing information on cart design and interface design and by providing guidance and feedback on the control system design as it progressed. 


\section{INTRODUCTION}

The West Valley Demonstration Project is sponsored by the United States Department of Energy (DOE) and is located at the former Nuclear Fuel Services reprocessing plant site at West Valley, New York. The primary objective of the project is to solidify high-level waste (HLW) stored in underground tanks into a form suitable for transportation and disposal. DOE has selected vitrification as the method of solidification and borosilicate glass as the waste form.

The existing HLW was produced from the chemical reprocessing of approximately $640 \mathrm{t}$ of spent nuclear fuels. The waste was discharged to underground storage tanks. These tanks currently hold a total volume of about $2165 \mathrm{~m}^{3}(572,000 \mathrm{gal})$ of liquid HLW.

After preliminary treatment steps, the liquid HLW will be transferred to the Vitrification Facility. The waste slurry will be continuously fed to the vitrification melter, where the waste and molten glass mixes and homogenizes. After some time, the glass will be poured into disposal canisters. The canisters will cool and be temporarily sealed for external decontamination. The seal will then be removed and replaced with a dust cover. The canister will then be ready for transfer to the former Chemical Process Cell (CPC) for interim storage.

The main purpose of the transfer cart is to transfer the empty canisters into the Vitrification Cell (VC) and filled canisters from the VC to interim storage in the CPC. Other future uses of the transfer cart have also been identified.

The design of the transfer cart has been a joint effort by West Valley Nuclear Services (WVNS) and Oak Ridge National Laboratory (ORNL). Generally, ORNL has been responsible for the design of the transfer cart control system, and WVNS has been responsible for the balance of the transfer cart design. The complete scope of the transfer cart control system design is defined in later sections of this report, which covers the design requirements and design description. In addition, this report documents design verification activities performed by ORNL during completion of the detail design. 


\section{DESIGN REQUIREMENTS}

The design requirements for the transfer cart control system were provided by WVNS via formal communication. These requirements are contained in two documents: (1) West Valley Demonstration Project, Design Criteria, General CTS Component Design Criteria, Doc. No. WVNS-DC-011, revision 4, revision date May 11, 1987, and (2) West Valley Demonstration Project Design Basis, Vitrification Facility Transfer Cart, revision 2, revision date November 26, 1991. Additional requirements specific to the control systenn design were documented in a formal communication from ORNL to WVNS at the beginning of the design. This section contains a summary of significant design requirements for the transfer cart control system.

\subsection{SCOPE}

The scope of the transfer cart control system includes all electrical and controls equipment needed for operation of the transfer cart. This includes on-cart controls, on-cart batteries, battery charging system electrical components, control signal transmission equipment, remote antennas, and operator controls.

\subsection{FUNCTIONAL REQUIREMENTS}

The transfer cart shall be designed to move a load of four filled canisters ( $10.4 \mathrm{t}$ ) plus the cart from the VC to the CPC with adequate reserve capacity. Additional capacity for loads up to $25 \mathrm{t}$ shall $\mathrm{b}$ p provided to handle vessels and load-test weights. The cart shall be designed to operate on the existing rail design between the VC and the CPC. The size of the cart and all components on the cart shall fit within all the existing design door openings. The cart shall be designed for individual drives for each of the four drive wheels to increase redundancy while decreasing maintenance difficulties. The cart shall be remotely operable from various ex-cell positions using general area remote closed-circuit television (CCTV) and shielded viewing windows. No CCTV cameras or monitors are required in the transfer cart design.

\subsection{ELECTRICAL}

The cart shall be powered with no trailing umbilicals. (This requirement ultimately means battery power and wireless controls. The cart must operate on the existing rail design, which has no provision for fixed power conductors. Also, because of door discontinuities and radiation levels in existing cells, new construction to add power and signal transmission in the floor is not feasible.)

The cart shall be capable of having the battery pack recharged without removing it from the cart. The charging location will be a permanent installation in the Equipment Decontamination Room (EDR). The connection and disconnection shall be automatic as the transfer cart passes 
through the charging location. The batteries should also be capable of being charged off the cart at a separate location.

The cart battery pack shall be designed and wired so that it can be removed and reinstalled remotely. The container for the battery pack should be sealed and decontaminable. The battery pack shall be designed so that it can be removed from its container and disposed of as nonradioactive waste.

\subsection{INSTRUMENTATION AND CONTROLS}

The cart on-board electrical controls shall be designed in an enclosure so that they can be removed and reinstalled remotely. All on-board controls shall be modularized as much as possible and be capable of remote removal as a unit using only the overhead crane and impact wrench. Two auxiliary remotely controlled power outlets shall be provided to allow use of dc powered tools from the transfer cart.

Battery voltage shall be monitored over the remote control data transmission system. The battery pack shall be instrumented to remotely indicate the state of battery charging and discharging. Motor current shall also be monitored over the remote control data transmission system for all four drive motors. The cart shall have capability to detect over/under current draw for the drive motors and be remotely reset if required.

Two control stations shall be providet to operate the cart. One station shall be located on the north wall of the VC, and an additional station sh the provided at the north CPC viewing window. Interlocks shall be provided to use one station at a time and not permit the cart to be operated from both stations at the same time. However, one control function shall be operable from either control station. Both control stations shall have an emergency kill control which shall be operable from either station at all times during operation.

Basic controls shall provide for controlled cart movement in either direction. All cart control switches shall be spring-returned to off. When the spring-return control switch is released and power is shut off to the drive wheels, the cart should stop within 6 in. at maximum speed with a full canister load. The operation of the cart shall be interlocked so that the cart cannot be energized while any of the following door control systems are energized; operation of the following doors shall also be interlocked so that they cannot be energized while the transfer cart is energized.

- Transfer tunnel to VC door

- Transfer tunnel to EDR doors

- EDR/CPC shield door

A logic function shall be provided which will initiate an audible warning when the transfer cart is being driven toward any door that does not have a fully open limit switch indication. The cart control system shall be designed to accommodate the existing penetrations for antenna placement.

\subsection{OPERATIONAL REQUIREMENTS}

The transfer cart operates in the VC, transfer tunnel, EDR, and CPC, as shown in Fig. 2.1. A rack with the capacity to carry four canisters will normally be installed on the frame of the cart. When it is necessary to move equipment other than the canisters, a new fixture(s) will be required in lieu of the rack. The charging location shall be located in EDR, where viewing of the cart is possible using the EDR crane-mounted CCTV. The cart will remain at the charging location until 


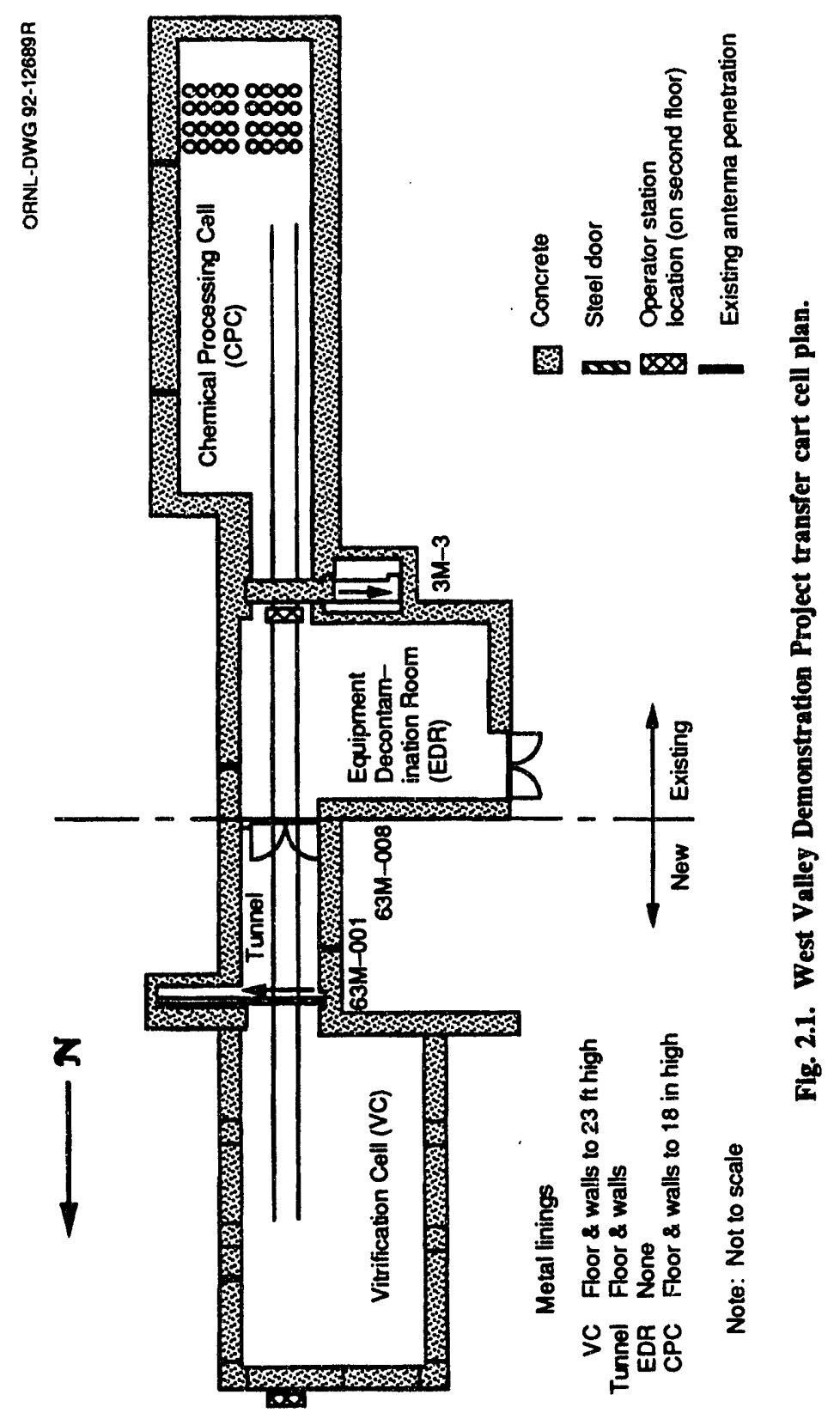


called upon for service. The empty canister loading station should be, if possible, at the same location in the EDR.

Normal operation of the transfer cart system is to move empty canisters into the VC and move full canisters from the VC to the CPC. The steps required to do this are summarized as follows:

1. When the canisters are ready for transfer, the cart will be taken from the EDR through th transfer tunnel to the VC. The operator will open the doors to the transfer tunnel, move the cart into the transfer tunnel, and then close the doors. The operator will then open the door to the VC and move the cart to the north end of the rails in the VC. The operator will then close the VC door.

2. The operator will load four canisters into the rack from an interim storage rack in the pit using the VC crane. This operation could take up to $2 \mathrm{~h}$.

3. The operator will open the VC door to the transfer tunnel and move the cart to the transfer tunnel and stop it. The operator will slose the VC door and then open the transfer tunnel doors to the EDR. The operator will then move the cart to the EDR, stop, and close the transfer tunnel doors. The operator will then, onen the EDR/CPC shield door and move the transfer cart into the CPC. Once the cart is in the CPC, the EDR/CPC shield door will be closed. During these steps the operator(s) could move about to the various viewing positions, as necessary, or the CCTV system could be utilized.

4. The operator will unload the canisters using the CPC crane. The operator will then move the cart back to the charging location in EDR and close all shield doors.

When moving new empty canisters into the cell, the operator will load four empty canisters onto the cart in the EDR. After loading, the cart will be moved through the transfer tunnel into the VC as described previously. After being unloaded, the cart will be moved again to the charging location in the EDR.

\subsection{ENVIRONMENT}

The design radiation ficld is $10,000 \mathrm{rad} / \mathrm{h}$ in the cell in close proximity of the canisters and diminishes to approximately $0.010 \mathrm{rad} / \mathrm{h}$ at the charging location in the EDR. Temperature will range from 20 to $120^{\circ} \mathrm{F}$, and relative humidity will range from 10 to $90 \%$. Nitric acid vapor content in the air will be up to $100 \mathrm{ppm}$ during off-normal conditions.

\subsection{MATERIALS}

The metals for all in-cell equipment shall adequately resist a decontamination process. Stainless steels are preferred. No uncontained organic materials shall be used on the cart. Wires and electrical components shall resist the radiation exposure or be shielded to provide a life of 5 years in the Vitrification Facility.

\subsection{MAINTENANCE AND REMOTE MAINTENANCE}

The system shall be designed to operate for a minimum of 5 years without maintenance. All materials used on the cart shall be designed for 5 years of operation without maintenance in a radiation environment resulting from the integrated dose. The total accumulated dose goal is $10^{7} \mathrm{rad}$. 


\section{SIGNAL TRANSMISSION ASSESSMENT}

The method vi communicating control signals to the cart is the most critical aspect of the overall design of the cart control system. Because of metal linings on the facility cell walls, radio frequency (rf) signal nuiling will result from multipath reflections of the transmitted if signal. Multipath reflections caus: amplitude degradation of the signal. The Instrumentation and Controls division of ORNL has woiked with similar systems in the past. During system conceptual design, an assessment of available options was undertaken and the best choice was selected for detail design. This section summarizes the assessment.

\subsection{OPTIONS}

Various options for the transmission system were examined in order to choose the best all-around system. The options included direct links, leaky coax systems, directive rf systems, and omni-directional rf links. The omni-directional transmission system was selected as the best system. The following sections discuss each of the systems that were examined and their respective advantages and disadvantages.

\subsubsection{Umbilical Cord}

The umbilical cord (or tether line) consists of an attached cable between a controlling station and the cart. The umbilical cord would avoid all radiated signal problems such as multipath and interference from other sources. However, the umbilical cord was not chosen because, in this application, there will be doors that are closed both behind and ahead of the cart. Umbilical cords had been used on earlier versions of the transfer cart, and there were frequent problems with the doors cutting the umbilical. Also, the requirements document for the project states that this option should not be used.

\subsubsection{Leaky Coaxial Cable}

The leaky coaxial arrangement consists of a series of cables, placed parallel to the tracks, that radiate energy through their shields. The receiving system on the cart obtains the desired information from this radiated energy. The leaky coaxial arrangement would be less susceptible to problems such as multipath and interference from other sources than would antenna-to-antenna systems. But, according to previous surveys, remote maintainability is a problem since the coaxial line rnust be segmented into removable lengths (of about $3 \mathrm{~m}$ ), which greatly adds to the number of electrical/mechanical connections that must be maintained. Also, since part of the Vitrification Facility includes an existing radioactively contaminated cell, new construction is not feasible. Remote installation of the cable would be required and would be extremely difficult. 


\subsubsection{Directive Antenna Link}

A directive antenna link consists of both receiving and transmitting antennas that radiate energy in a relatively narrow beamwidth. This arrangement requires that the antennas be aligned in order to establish a transmission path. A directive antenna link with a beamwidth of only a few degrees can be used to greatly reduce the problem of multipath reflections. The transmission systems that operate at frequencies above about $1 \mathrm{GHz}$ allow a greater information bandwidth. At these frequencies, parabolic dishes, which are inherently directive antennas, are normally used. A directive antenna link requires equipment such as parabolic dishes and steering mechanisms. The dishes would take up more space on the cart than would antennas such as monopoles, especially at frequencies of about $10 \mathrm{GHz}$. The antenna systems would require steering because a fixed directive link is not allowed because of the interfering doors. This steering would require much more complex hardware, cost, and maintenance. If even higher frequencies such as 90 to $100 \mathrm{GHz}$ are selected to reduce size, the hardware would cost an estimated $\$ 60,000$ more than a $10-\mathrm{GHz}$ system.

\subsubsection{Omni-directional Antenna Link}

The omni-directional antenna link, which has been selected for this project, consists of receiving and transmitting antennas that radiate energy equally in all directions. The omnidirectional antennas are normally quarter-wave monopoles or half-wave dipoles which are very simple both mechanically and electrically. Since they radiate equally in all directions there is no need to steer the antennas. At a frequency of about $1 \mathrm{GHz}$ (which would be sufficient for the needed information bandwidth of this project), the antennas are about 6 in. long or less. The antennas and associated hardware are standard equipment and are relatively inexpensive. The omni-directional antennas combined with the metal-lined walls, however, produce many multipath reflections, which degradate the received signal. These types of antennas are normally only used at frequencies of a few gigahertz or less; thus, they limit the information bandwidth that can be used.

\subsection{LITERATURE SURVEY}

The literature that discusses multipath reflections, especially in metal-lined cells, has been researched in order to give a more thorough basis for omni-directional transmission. The report written by Burgess et al. ${ }^{1}$ discusses the allowable bandwidth for a given carrier frequency in a metal-lined cell. The theory deals with a metal-lined tunnel similar to the transfer tunnel through which the West Valley cart will travel. The equations show the different modes that propagate in this highly reflective environment. Although there is significant distortion because only relatively narrow bandwidths are needed for the required data rates, the distortion is not a problem. Transmission in the larger cells where the cart is farther from the reflecting surfaces will not be as critical in nature, although there will still be attenuation due to multipath reflections.

\subsection{TESTS}

Tests were performed to show that a bandwidth of about $1 \mathrm{MHz}$ on a carrier of $1 \mathrm{GHz}$ would be feasible inside a structure such as the transfer tunnel, since its metal floors and walls make it the worst case area for multipath degradation. 


\subsubsection{Test Procedure}

The tests performed transmitted a carrier frequency of about $1 \mathrm{GHz}$ in a completely metallined room that is $4.58 \mathrm{~m}$ long $\times 3.65 \times \mathrm{m}$ wide $2.46 \mathrm{~m}$ high (i.e., all dimensions are much greater than a wavelength). Both directive and omni-directional transmitting antennas were used with a monopole receiving antenna. Both frequency modulation (FM) and amplitude modulation (AM) were used, and the output of a receiver was examined to note the distortion of the transmitted information as well as the signal strength. The transmitting and receiving antennas were placed in various combinations of locations both near the walls and in the middle of the room. The antennas were also placed so that there was not a line-of-sight transmission path.

An impulse generator was used to generate the broad spectrum, and a spectrum analyzer was used to show the frequency response of the antenna-to-antenna link in the metal-lined room. The impulse generator produces energy of an equal amplitude across a large span of frequencies. The received frequency spectrum from a radiated impulse shows the characteristic frequency response of the transmission system.

\subsubsection{Test Results}

The tests indicate that FM transmission of about 1-MHz bandwidth is feasible for a metallined room of dimensions much greater than one wavelength at about $1 \mathrm{GHz}$. Also, it was noted that when the antennas were placed $30 \mathrm{~cm}$ or less from the wall, nulls would occur over various portions of the frequency band but never over more than one-third of the desired spectrum. Thus, using a combination of wideband FM as well as a diversity receiving system (explained later) should be adequate for data transmis :ion.

\subsection{DESCRIPTION OF PROPOSED SYSTEM}

\subsubsection{Antenna System}

As shown in Fig. 3.1, the basic system is based on omni-directional transmission. The stationary antenna will be a dipole, and the mobile antenna system will consist of two dipole antennas mounted on the cart. Although there will be many reflections from the stainless steel cell walls, the system can be designed to overcome this problem. The reflections are called multipaths and cause amplitude degradation of the signal. The multipath problem is overcome by employing one or more of the following: (1) diversity receiving in which more than one antenna is used, (2) filtering that minimizes the data transmission error rate, and (3) wideband FM that spreads the information over a larger bandwidth. ${ }^{2}$

The carrier frequency will be $915 \mathrm{MHz}$, which will allow the use of standard hardware and antennas that are practical in size. (Dipoles would be $16.4 \mathrm{~cm}$ long, and monopoles would be half the length of the dipoles.) The carrier frequency will be in the industrial, scientific, and medical assigned range of 902 to $928 \mathrm{MHz}$.

\subsubsection{Communication System}

The communication system will use a bandwidth of about $1 \mathrm{MHz}$ with FM. This relatively narrow bandwidth allows the use of omni-directional transmission in a reflective environment. This bandwidth should be sufficient to accommodate a $1-$ to $2-\mathrm{kHz}$ data rate, some encoding for error correction, and some wideband spreading of the transmitted energy to better utilize the transmission channel in a reflective environment. FM will be used instead of AM since the 


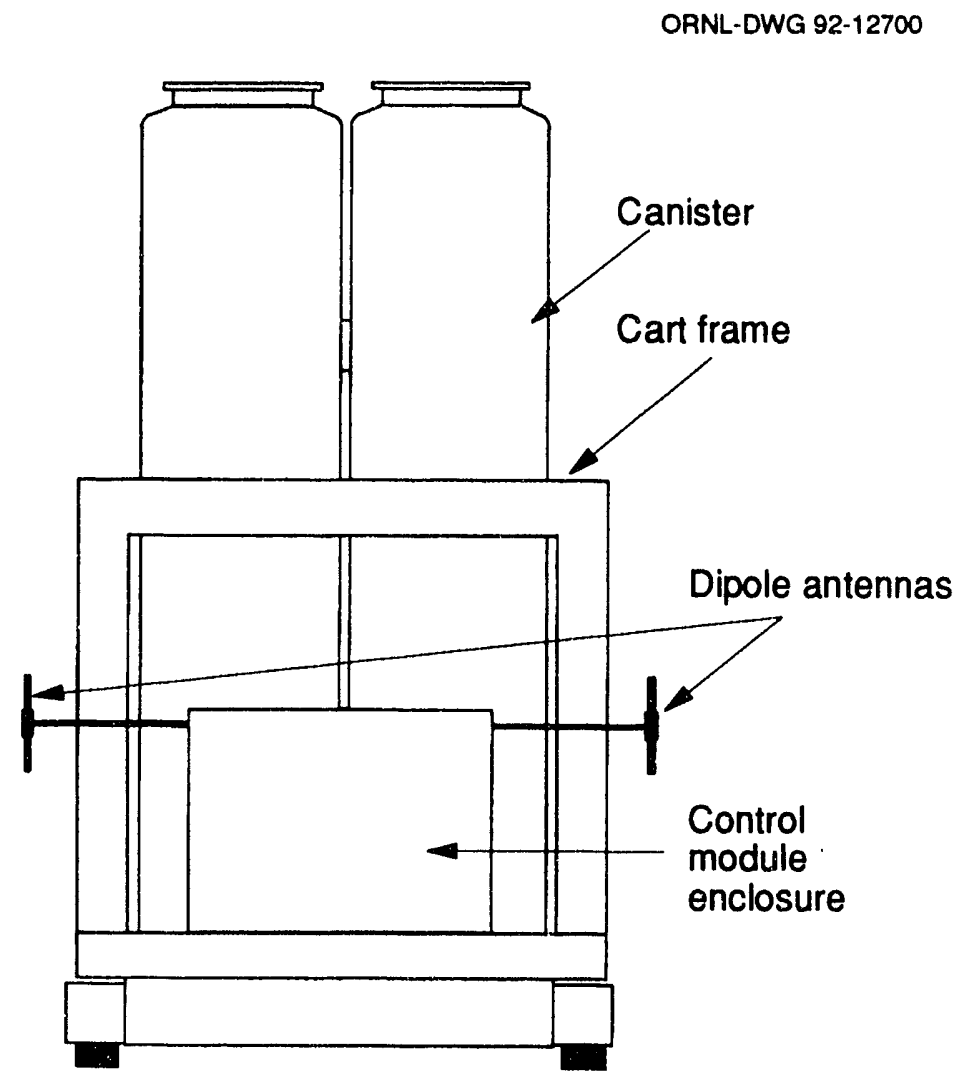

Fig. 3.1. Mounting of dipole antennas on cart.

reflections will produce an effective AM distortion of the signal to which FM detection will be less susceptible. Also, FM is less susceptible to other noise sources such as welding equipment. As mentioned before, there will be two dipole antennas on the transfer cart. These antennas will be placed at the extremities of the cart envelope so that line-of-sight transmission will be maintained as much as possible. The redundancy is two-fold in that it allows the receivers to pick either antenna based on which one is receiving the stronger signal and it provides a backup in case one antenna becomes inoperative. The signals will be compared, and the stronger will be used for obtaining the information. 


\section{SYSTEM OVERVIEW}

The communications system introduced in the previous section, although a major component, is part of a larger overall system. Figure 4.1 shows the major equipment in this design. Major equipment items include the transfer cart control module enclosure, the battery charger, the north and south operator's pendants, the engineer's console, and facility antennas. Facility systems are discussed in Sect. 5; the communications system for both the facility and the cart, in Sect. 6; the cart electronics system, in Sect. 7; mechanical features of the cart control module enclosure, in Sect. 8; and software of both facility and cart systems, in Sect. 9.

The design is a combination of off-the-shelf components and specially designed equipment. Early on in the design we recognized that a radiation-tolerant, battery-powered communications system did not exist commercially and therefore must be specially designed. Special interfacing cart electronics were also designed. However, a commercially available embedded controller was identified and radiation tested for use on the cart. On the facility side, the other side of the communications system to match the cart side was specially designed. A unique requirement (the ability to switch charging polarity of the cart batteries) led to a specially designed battery polarity interface circuit. The control pendants, although based on a commercially available hand-held pendant, have a specially designed circuit board and faceplate decal. The programmable logic controller (PLC), the engineer's console computer, and the battery charger are all commercially available.

Drawing Q-6340-001 provides an overview of system functionality, as expressed in Instrument Society of America symbology. ${ }^{3}$ The tag names on the drawing show how individual points are referred to at the engineer's console. All design drawings can be found in Appendix $\mathrm{E}$. 


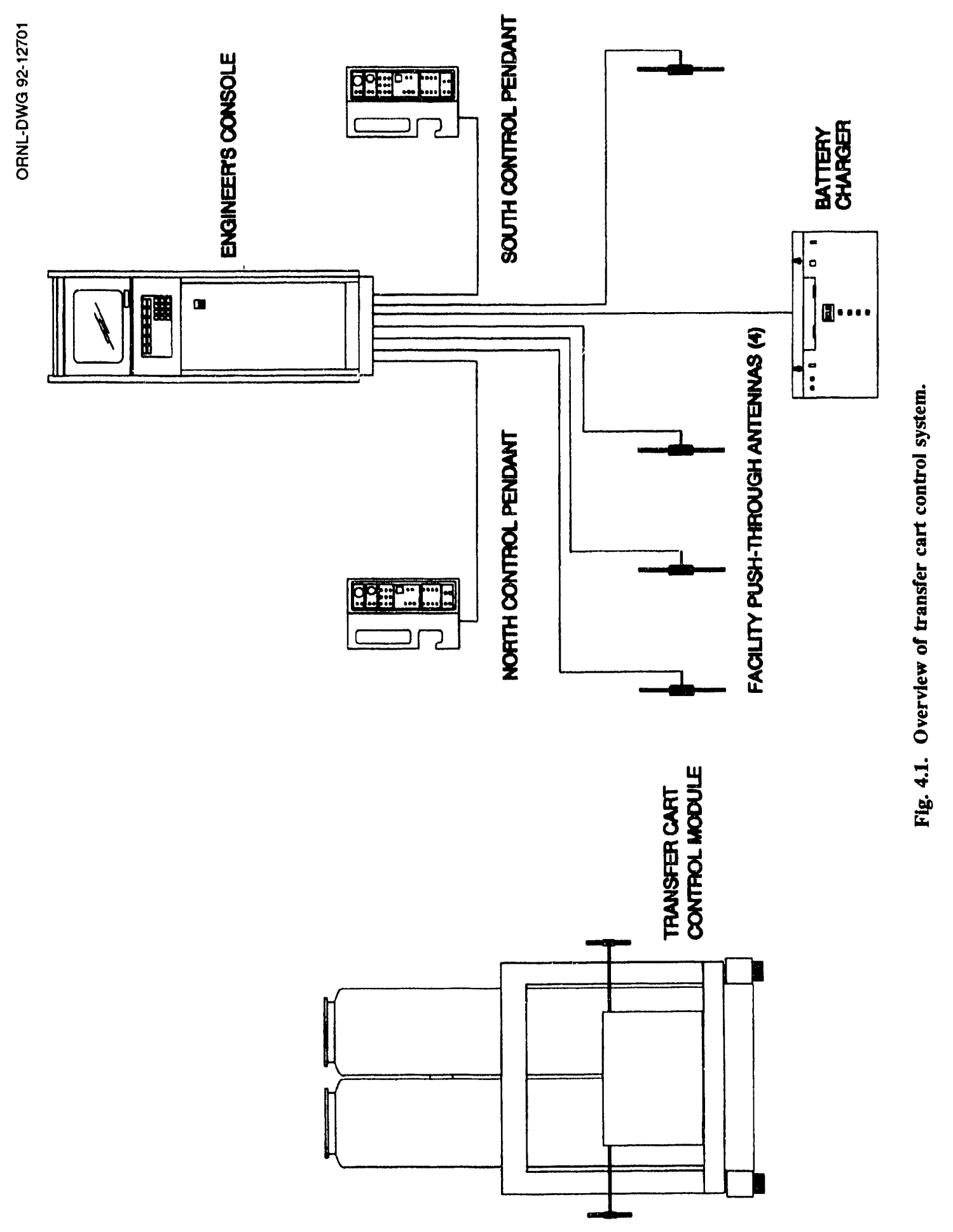




\section{FACIITTY SYSTEMS}

Facility systems include the control pendants, the engineer's console (which houses the engineer's console computer, the PLC, communications equipment, and ancillary equipment), the battery charger and its interface, and cell door limit switch interfaces. The facility communications system is described in Sect. 6. The descriptions of the remaining systems are included in this section.

\subsection{CONTROL PENDANTS}

Operation of the cart will be controlled by one of two hand-held control pendants (see Fig. 5.1). One pendant will be located near the north viewing window of the VC, and the other will be located near the north viewing window of the CPC. The functionality of both are the same. The control pendants consist of switches, light-emitting diodes (LEDs), and an audible alarm. The switches and LEDs are divided among functional blocks on the front face of the pendant. The audible alarm is mounted on the top side of the pendant. The push-button switches are used for operator command inputs, and the LEDs are for visual status indication. An oversized emergency stop button is provided for stopping the cart and battery charger from either pendant. A threeposition, return-to-center rocker switch is provided for cart drive. ORNL will provide a metalphoto decal which will be placed on the face of the Rose Enclosures model 2950 hand-held pendant. Drawings Q-6340-120 through Q-6340-123 document the pendant design.

The cart control system operates in two basic modes: (1) battery charging and (2) cart operation. In the battery charging mode, the cart is disabled completely; sending ، mmands to the cart will have no effect, and the cart will not report status of any of its normally monitored variables. In the cart operation mode, the battery cannot be charged unless it is located at the charging shoes (described in Sect. 5.4). A description of the cart operation using the control pendants is included in Appendix F.

A printed circuit board (s:e Dwg. Q-6340-123) provides a backup for mounting LEDs and push-button switches. Other components mounted on the board include resistors in series with the switches to provide debounce filtering of the switch contacts (see Dwg. Q-6340-122). Filtering is provided through the resistor and capacitance of the multiconductor cable. After instillation, additional capacitors could be added, if necessary, at the engineer's console side of the cable for additional filtering. The board also houses two capacitors in parallel with the audible alarm to reduce high-frequency noise from appearing on the multiconductor cable that connects the pendant with the engineer's console. The filtering is used to reduce the possibility of spurious noise appearing as an input to the PLC. Finally, compression terminal blocks are mounted on the circuit board to terminate the multiconductor cable.

Internal components not mounted on the printed circuit board include the audible alarm, the emergency stop switch, and the station select key switch. A metal-photo decal will be provided by ORNL to insert in the front recess of the pendant. In addition to showing the functionality of the pendant, the decal will be used as a guide for drilling the LEDs and switch holes. 


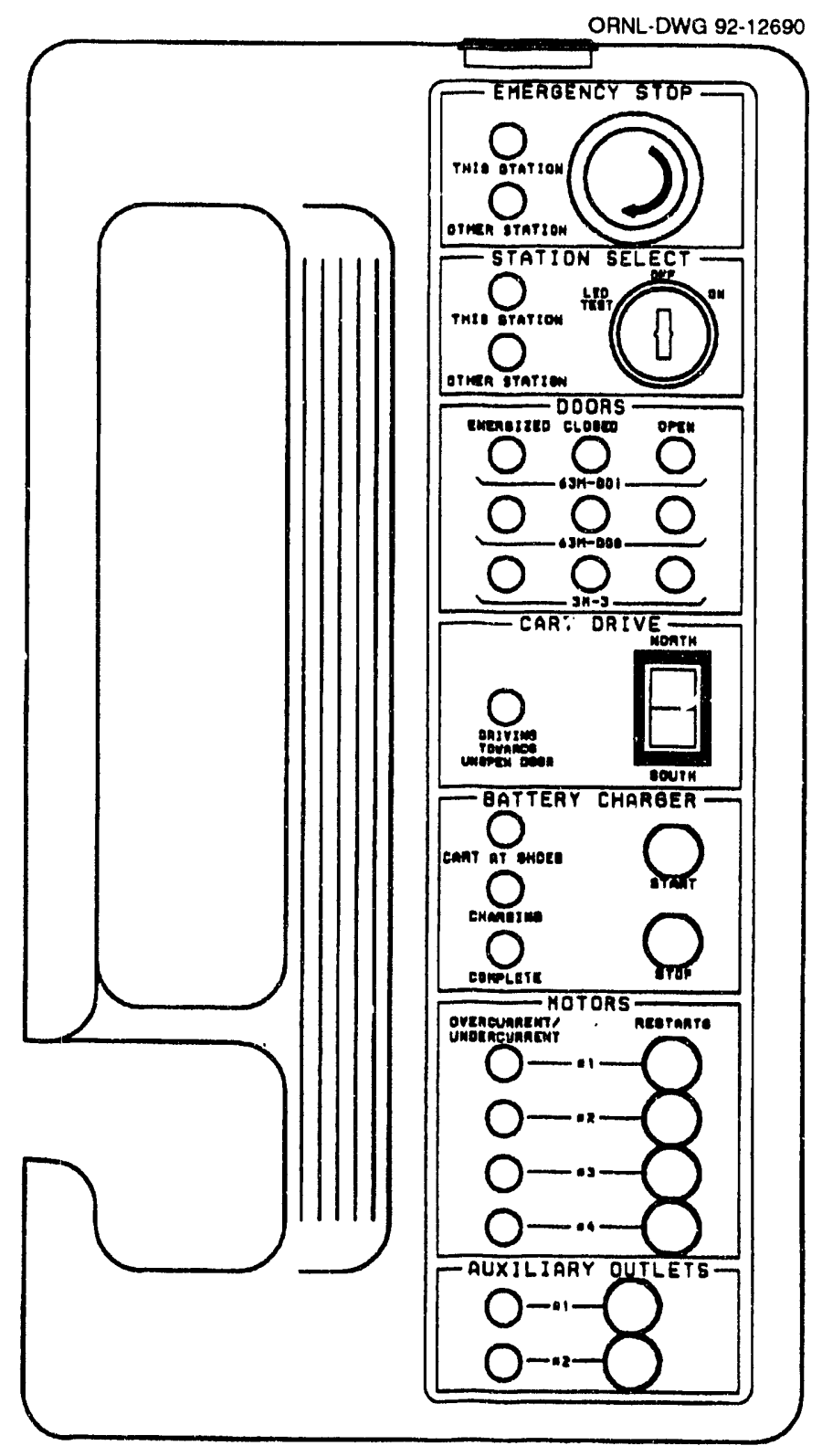

Fig. 5.1. Operator's control pendant.

When not in use the pendant can be hung from its junction box, as shown in Dwg. Q-6340125. A 50-conductor cable will interconnect the control pendant to the PLC, which is mounted in the engineer's console, as described in the next section. Typical wiring of the pendants is shown in Dwg. Q-6340-126. 


\subsection{PL.C}

The PLC selected for this application is an Allen-Bradley PLC 5/15. Because of previous experience with Allen-Bradley at the West Valley site, West Valley personnel requested that Allen-Bradley be used as much as possible for control hardware. In the Allen-Bradley family, the PLC 5/15 model provides the lowest price per functionality ratio for this application. Besides the typical digital interfacing, this application is somewhat unique for a PLC in that it requires interfacing to the battery charger through an RS-232 link. The Allen-Bradley 5/40 model features direct interfacing to RS-232, but its much higher cost is prohibitive. Through the present architecture, the $5 / 15$ interfaces directly to the Prolog communications controller (which is described in Sect. 6.8) using the Prolog 7514 interface card. The PLC uses the Prolog RS-232 interface to communicate to the battery charger. An early decision to base the rf boards on the STD bus also precluded using the Allen-Bradley PLC for the communications controller. Furthermore, high-speed communications tasks must be coordinated closely by the communications controller and may not have been possible in the PLC ladder logic because of the complicated functionality (see Sect. 6.8).

The PLC is the heart of the sontrol system through which all control activities are coordinated. It will interface to both control pendants through digital input/output (I/O) modules, to the communications controller over the PLC's remote I/O link and a Prolog 7514 Allen-Bradley interface card in the communications controller, and to the engineer's console computer over the Data Highway Plus link and a Sutherland-Shultz 5136 SD interface card in the engineer's console computer. The PLC also interfaces to the cart orientation key switch, to the facility doors, and to the enable/disable input of the battery charger interface through the PLC's digital I/O.

The PLC will physically reside in the engineer's console in a 19-in. rack. Rack modules include the PLC 5/15 (1785-LT) processor with a 1785-MJ EEPROM, four 1771-OBD digital output modules, three 1771-IBD digital input modules, and a 1771-P4S power supply. The EEPROM module provides retention of the operating program and current values of variables in the event of a power loss.

Special ladder-logic software which will run on the PLC is described in Sect. 9. A listing of the logic should be referred to for further details of the ladder logic. The engineer's console computer will be used to modify, download, and monitor ladder logic running on the PLC.

\subsection{ENGINEER'S CONSOLE COMPUTER}

The engineer's console computer resides in the engineer's console assembly, as shown in Fig. 5.2. The engineer's console computer will be the engineer's window into the control system. Through th. computer, an engineer can monitor cart activity and data, whether current or historical, ard operational status of the control pendants, doors, and battery charger. A commercial man/machine interface software package, Intouch, has been used to develop the engineer/control system interface. In addition, the computer can modify and download ladder logic in the PLC. It can also modify, compile, and download programs to the Prolog communications controller. A switch in the back of the engineer's console will allow the battery charger to interface directly to the engineer's console computer through one of its serial communications ports. In this mode, configuration parameters of the battery charger can be interrogated and changed and it would be possible to operate the battery charger in this mode even in situations when the control pendant, the PLC, or the communications controller had failed. The engineer's console computer will be capable of trending any of its inputs and providing alarm outputs based on the input value. It will also be capable of performing mathematical and logical operations on the cart data. 


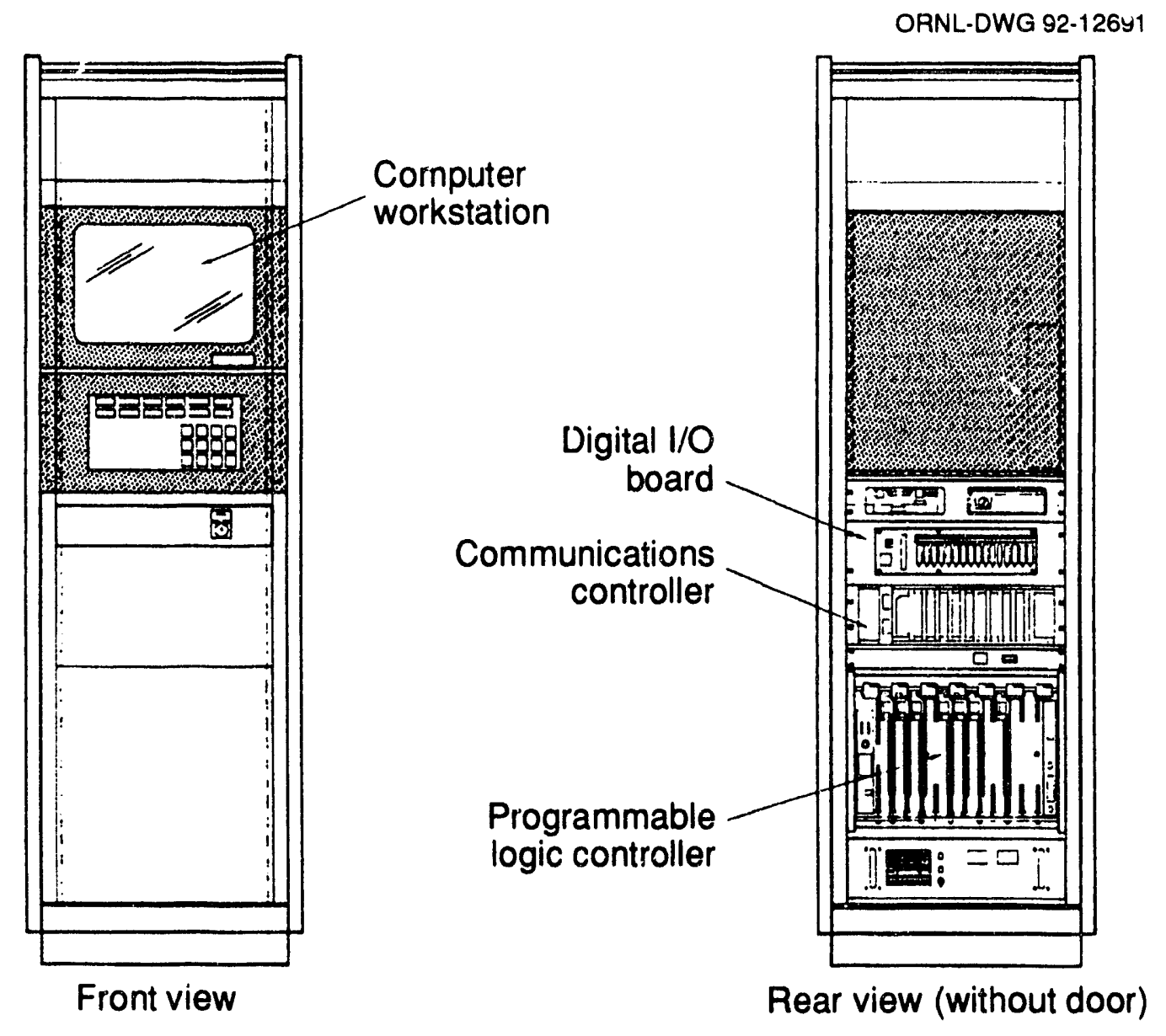

Fig. 5.2. Engineer's console.

The computer will be using the Windows operating environment. The Intouch man/machine interface software operates exclusively on this platform. Furthermore, the terminal program supplied with Windows can be used to communicate with the communications controller or the battery charger while Intouch is logging data from the cart. Teledenken ladder logic software was used to develop the prototype system at ORNL. Because the Teledenken software does not operate under the Windows platform, a Windows-based ladder logic development software package should be used to take advantage of the Windows operating environment. ICOM is a vendor of Windowsbased ladder logic development software for the Allen-Bradley PLC; however, it is not clear whether ICOM supports the Sutherland-Schultz 5136 SD interface card. Both the man-machine interface software (Intouch) and the ladder logic software use this interface card. Furthermore, the ladder logic software would have to be converted to use the ladder logic developed for the prototype system, and both systems would have to be able to convert to or from the Allen-Bradley 6200 series standard. Because none of these conversions have been attempted, the conservative choice would be to use Teledenken for the final system and run it as a nonWindows application on the engineer's console computer.

A Nematron model IWS-5386DX is specified for use as the engineer's console computer; rowever, we suggest that the latest technology processor at the time of procurement be bought, as technology in this field changes rapidly. The Nematron is an industrially rugged computer with 
a shock-mounted disk drive, NEMA 12 sealing, and optically isolated communications ports. It has a 19-in. VGA color monitor and an $80386 \mathrm{DX} 25-\mathrm{MHz}$ processor. The unit should be bought with the highest capacity hard disk drive at the time of procurement, at least $8 \mathrm{MB}$ of random access memory (RAM), an 80387 math coprocessor, and a 3.5 -in. diskette drive. Because the unit will be operating under the Windows environment, a trackball is recommended as a pointing device. However, as presently configured, no trackball was specified for the system. All of the Windows features should still be accessible through keystrokes; if, however, a trackball is desired, options should be investigated for interfacing a trackball to the unit. A bus interface should be used since two of the computer's serial ports are being used already.

The computer is equipped with a front-panel function keyboard for routine operations. More intense diagnostic or maintenance operations can be performed by folding down the panel, which exposes a full-function QWERTY-style keyboard.

The computer interfaces to the PLC with a Sutherland-Schultz 5136 SD card located in one of the computer's expansion slots. Both Intouch and the ladder logic development software (Teledenken for the prototype) use this card for hardware interfacing. If a different ladder logic development software package is bought, it must be compatible with the Sutherland-Schultz card.

See Dwgs. Q-6340-102 through Q-6340-106 for details of the engineer's console assembly. See Sect. 9 for a description of the engineer's console man/machine interface software.

\subsection{BATTERY CHARGER}

The battery charger specified and tested for this project is an Exide ERBC 24/30. It is a unique battery charger in that it features a microprocessor for interfacing to the control system. Start and stop commands are issued to the battery charger, and status variables for battery charging current, voitage, and temperature are retumed to the control system. The Prolog communications controller is the primary interface between the control system and the battery charger. However, a polarity interface circuit is also located between the battery charger and the batteries. The primary purpose of this circuit is to reverse charging polarity when the cart has been reversed on the tracks. Another key feature of the interface circuit is that it provides an emergency stop function that is available both locally and through the control system. See Sect. 5.5 for a description of this circuit. A second identical battery charger will be provided to maintain a spare battery in a float charge condition.

Connection of the battery charger to the cart occurs through a set of spring-loaded shoes designed by WVNS. The charge shoes will be located on the floor between the cart track rails and are designed to interface with a set of charging plates mounted on the bottom of the cart control module enclosure. A description of the circuitry connected to the cart-mounted charging plates is given in Sect. 7.3. A limit switch, to be provided by WVNS, will indicate when the cart is in the charge location. A typical charging sequence is described in Appendix F.

\subsection{BATTERY CHARGER INTERFACE CIRCUIT}

As described in the previous section, the battery charger interface circuit provides two major functions. First, the interface allows the polarity of the cart charging shoes to be reversed, and second. the interface provides an emergency stop function physically located near the battery charger: The charger interface is constructed in a Hoffman continuous hinge clamp cover box which will be mounted above the EDR near the battery charger. The interface uses solid state switches to control charge shoe polarity and to interrupt the charge circuit in the event of an 
emergency stop. Two control inputs, polarity and on/off control, from the engineer's console pass through a terminal block mounted in the interface box to drive circuits located on the battery charger polarity manager printed circuit board. The drive circuits control the state (on/off) of four independent high-current solid state switches and four independent low-current switches, as shown in Fig. 5.3. The drive circuits also provide for proper switch sequencing and an "all switches off" emergency stop condition. The high-current switches control the actual charge output lines from the battery charger, and the low-current switches control the charger voltage sense lines.

Assembly and wiring diagrams for the battery charger interface are given in Dwgs. Q-6340131 and Q-6340-132 respectively. A schematic of the battery charger polarity manager circuit board is given in Dwg. Q-6340-135. The battery charger polarity manager printed circuit board layout and parts list are given in Dwgs. Q-6340-136 and Q-6340-137 respectively.

\subsection{DOOR INTERFACES}

Each door will be monitored for three conditions: (1) door open, (2) door closed, and (3) door energized. Dry switch contacts must be provided by WVNS to interface to the I/O system which will power each circuit with a nominal $24-\mathrm{V}$ power surply. In addition, the PLC is capable of disabling all doors from operating when the cart is operating. Three outputs are provided from the PLC, which must be interfaced to the door actuation circuit using interposing relays designed by West Valley.

ORNL-DWG 92-12725

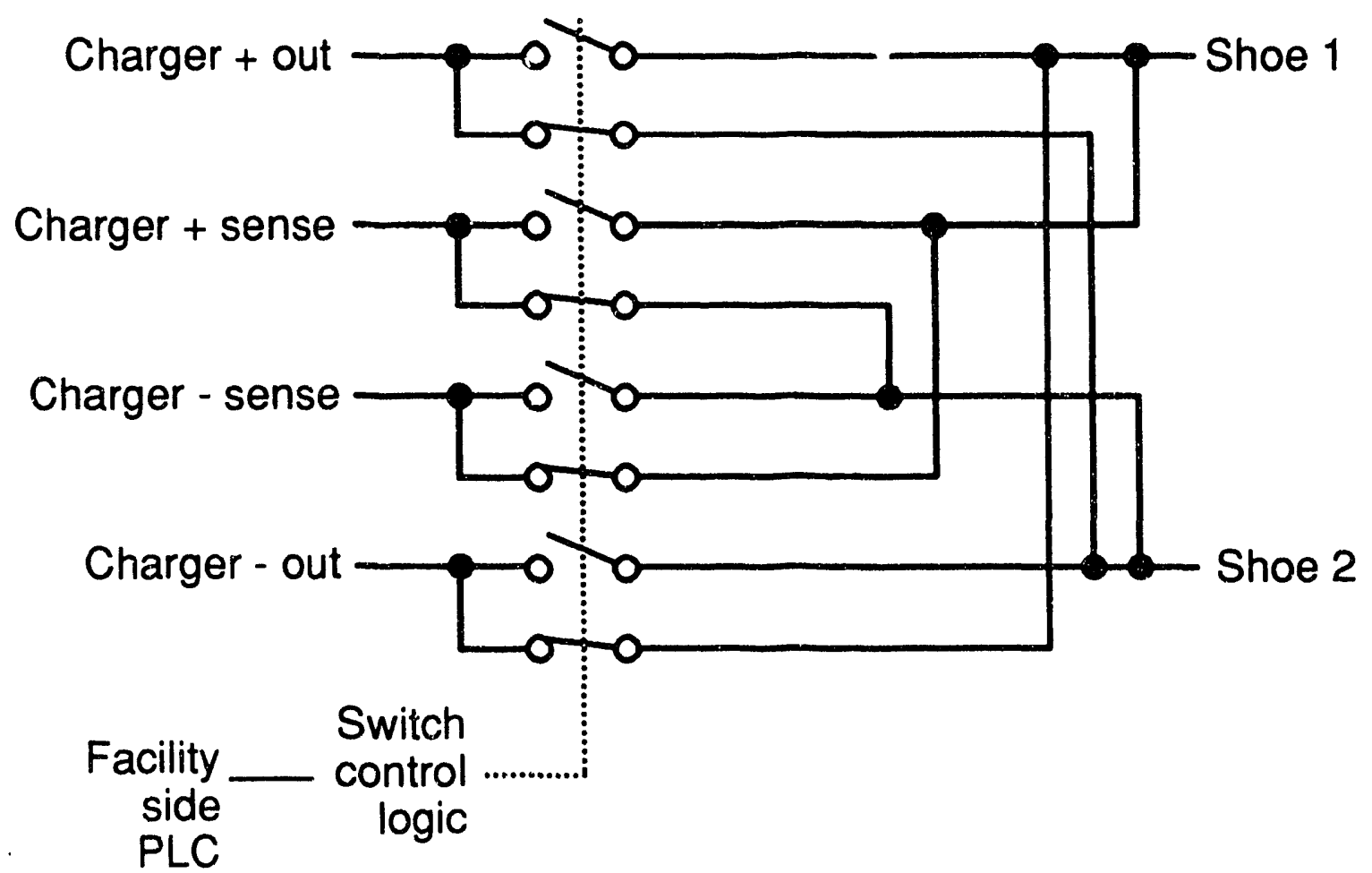

Fig. 5.3. Charger polarity control switching. 


\section{COMMUNICATIONS SYSTEM DESIGN}

The communications system will provide a wireless, bidirectional, digital data link between the facility and the transfer cart. Because cell walls and floors contain a significant amount of metal lining, signal multipath will occur. The communications system is designed to overcome problems associated with multipath reflections such as signal nulling at node cancellation locations. The system employs two cart antennas so that if one antenna is positioned in a null, the other antenna will be selected in the receive mode. Additionally, wide-band frequency modulation is used to avoid problems associated with zarrow-band signal attenuation. This technique allows a portion of the spectrum to be lost to multipath while retaining much of the signal energy. Further multipath immunity is introduced through multiple facility antennas required for the cart to travel among four cells, each of which may be isolated by doors. While the cart is being driven, the door between the originating cell and the destination cell will be open and two facility antennas will be available to communicate with the cart. The system will automatically select the facility antenna with the stronger received signal. Additionally, a manual override allows an

engineer at the engineer's console to inhibit any cell transmitter that genierates multipath while the connecting doorway is open. Rationale for the selection of the design is presented in Sect. 3.

A major concem in the hardware design of the cart communications system is that equipment located on the cart must withstand a total integrated radiation dose of $10^{7} \mathrm{rad}$. The communications system was designed around components of known radiation tolerances based on previous radiation testing experiments and operating experience gained from other designs.

The following sections give general descriptions of the various circuits used in the facility and cart transceiver systems. Refer to Dwgs. Q-6340-150 and Q-6340-250 for block diagrams of the facility communications system and the cart communications system respectively. Block diagrams of each circuit are included in this section, and the detailed schematic diagrams are provided in Appendix E.

\subsection{FACILITY TRANSMITTER CIRCUIT}

The facility transmitter circuit is responsible for converting the digital data from the communications controlier computer to an FM if carrier and distributing the of to the transmit amplifiers at each of the four cell locations. A block diagram of the facility transmitter is shown in Fig. 6.1. The circuit acquires a digital message packet from the communications biphase encoder/decoder board. The biphase signal is processed through a modulation amplifier that allows adjustment of both the if carrier frequency and the FM signal deviation. The modulation amplifier drives a voltage-controlled oscillator (VCO) that has a carrier frequency of $915 \mathrm{MHz}$ in the industrial, scientific, and medical (ISM) band. Deviation of the carrier is adjusted to $\pm 10 \mathrm{MHz}$ around the carrier.

The VCO output is buffered and amplified before being split into four identical signal channels. Each channel is amplified to a level of approximately $+7 \mathrm{dBm}$ (decibels referenced to $1 \mathrm{~mW}$ ) to drive a coaxial cable to the facility transmit/receive board at each of the four cell 


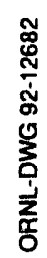

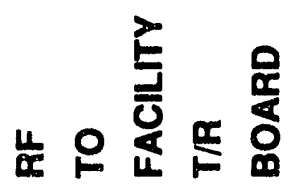

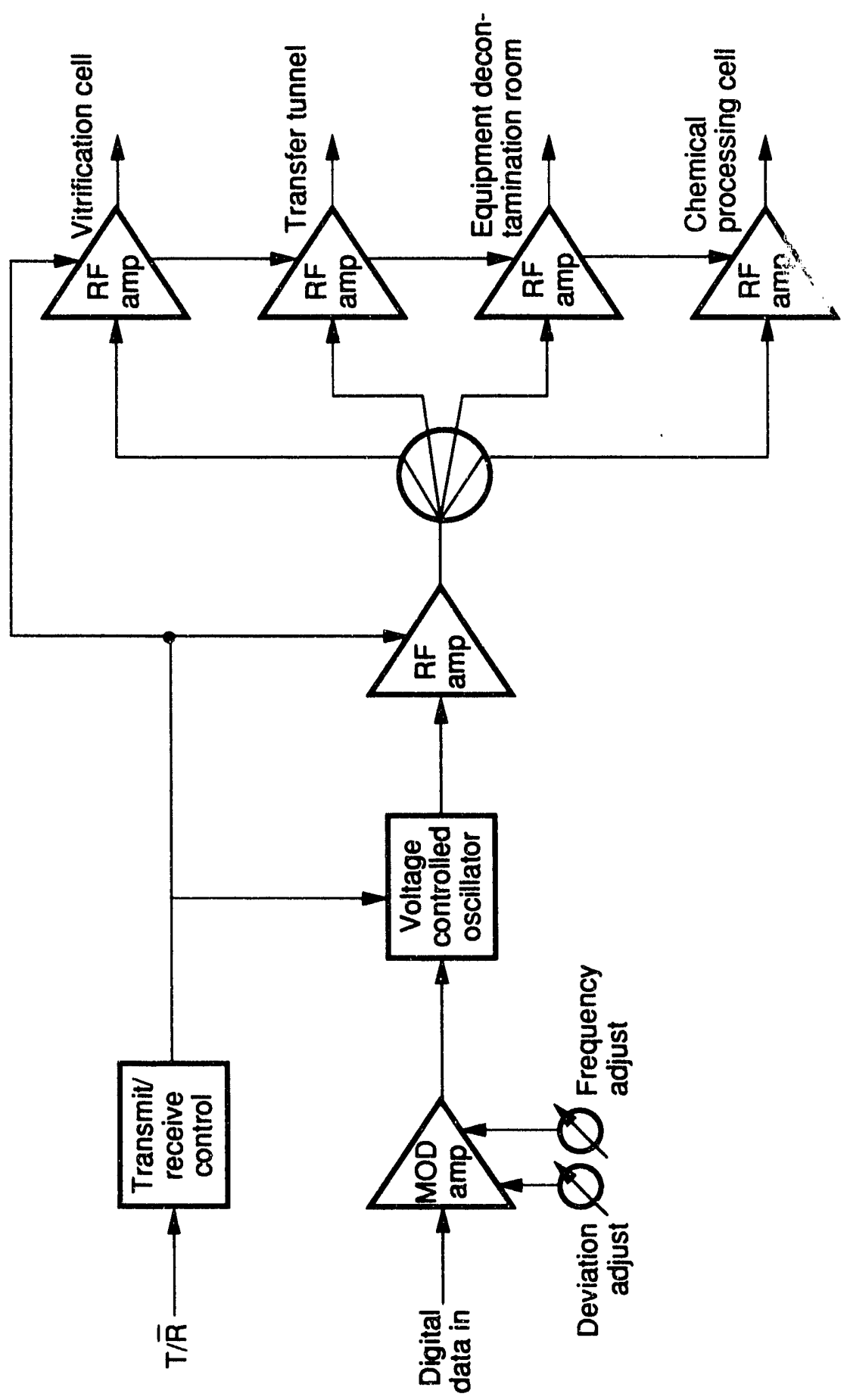


locations. A transmit/receive control signal is supplied by the facility communications microprocessor. This control signal activates the VCO and rf amplifiers during the transmit mode and deactivates them during the receive mode. A schematic diagram for the facility transmitter is given in Dwg. Q-6340-170. The circuit board is housed in a card cage in the engineer's console.

\subsection{FACILITY TRANSMIT/RECEIVE CIRCUIT}

The facility transmit/receive circuit provides the power amplifier drive to the antenna during the transmit mode and functions as the receiver preamplifier in the receive mode. Figure 6.2 shows a functional block diagram of the circuit. A control signal from the communications controller through the Opto 22 digital I/O panel (described in Sect. 6.8) determines whether the circuit functions in the transmit mode or in the receive mode. This control signal is brought to the cell interface as a 24-V signal on a twisted-shielded pair line and is buffered through an optical isolator for noise and transient immunity. The optical isolator drives a control circuit that powers the transmit amplifier during the transmit mode and powers the receive amplifiers during the receive mode. The fail-safe condition for loss of control places the system in the receive mode. The system is also placed in the receive mode during an emergency stop condition to eliminate the possibility of transmitting drive commands to the cart in the emergency stop mode.

In the transmit mode, the facility cell interface is supplied with a $915-\mathrm{MHz}$ FM signal from the facility transmitter board. The rf signal is amplified through a power amplifier and applied to an if circulator that routes the output to the cell antenna. The facility transmitter supplies approximately $100 \mathrm{~mW}$ of $\mathrm{rf}$ power to the antenna but is capable of driving higher levels if required.

During the receive mode, if energy from the cart transmitter is received by the facility antenna and is applied to the circulator. The circulator routes the signal through an rf switch to a low-noise amplifier circuit. The signal is further boosted through a driver amplifier (a total amplification of $40 \mathrm{~dB}$ ) that supplies the signal through a coaxial cable to the facility antenna switch in the engineer's console.

Electronics for the facility transmit/receive circuit consist of both a printed circuit board and connectorized if components. The optical isolator, local power supply regulators, and power switching transistors are located on the circuit board. The rf transmit power amplifier, circulator, rf switch, and receive amplifiers are connectorized modules interconnected by SMA coaxial cables. Power and control for each of these modules is provided from the printed circuit board.

Identical transmit/receive modules will be located on the opposite side of each cell wall from their respective cell antennas. Antennas will be located in the VC, the tunnel, the EDR, and the CPC. Each module connects to the communications controller microprocessor in the engineer's console through a $24-\mathrm{V}$ power cable, a control cable, a transmit coaxial cable, and a receive coaxial cable. The four modules are normally operated together in either the transmit or receive modes. The communications microprocessor, on command from an engineer at the engineer's console computer, can inhibit the transmit command to any cell, allowing a transmitter to be taken off line should communications problems occur due to multipath from two antennas when the cart is in the doorway between two cells.

The schematic diagram for the facility transmit/receive circuit is shown in Dwg. Q-6340-175. Drawing Q-6340-151 provides the assembly and parts placement for the printed circuit board and connectorized components of the circuit. 


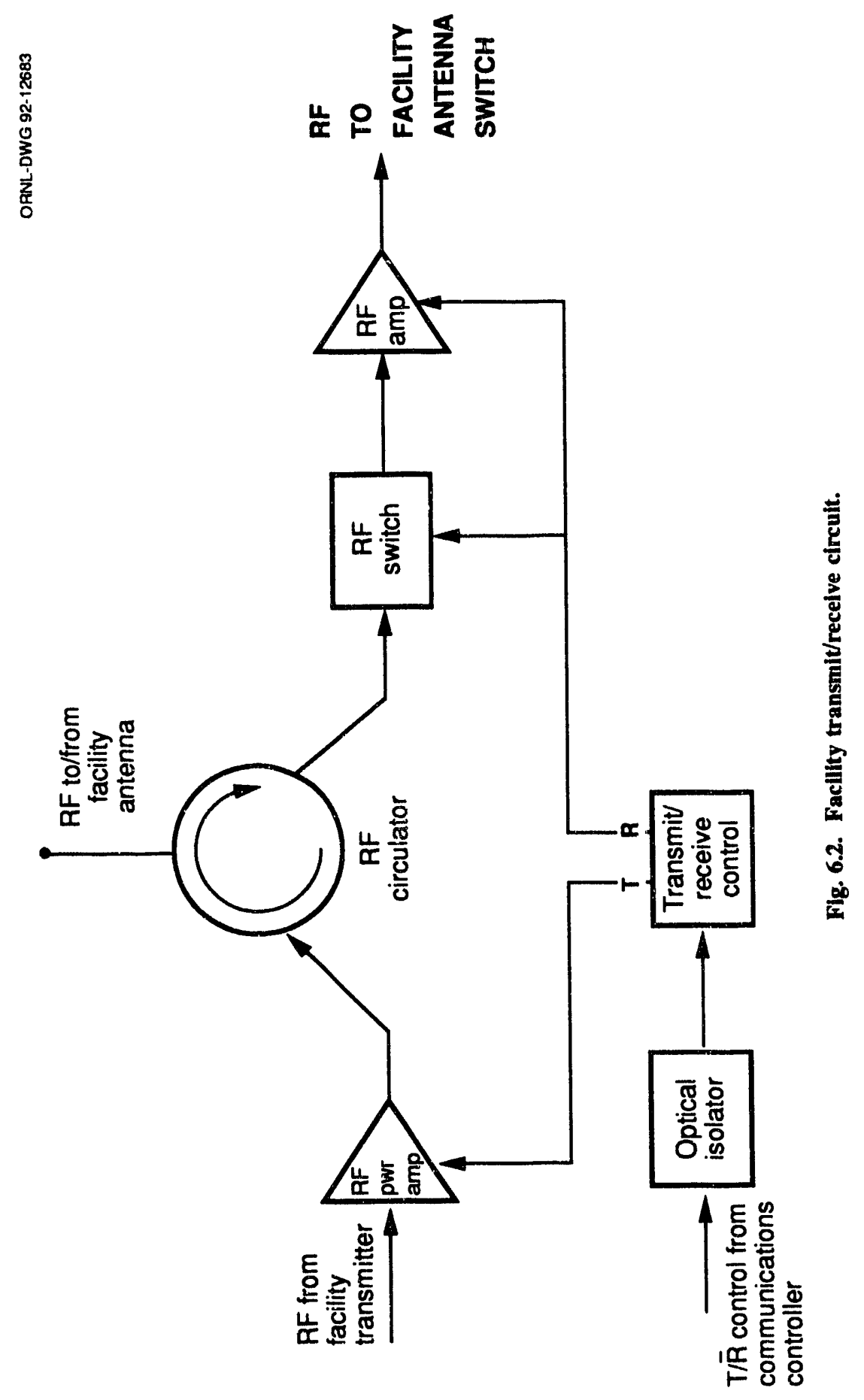




\subsection{FACILITY ANTENNA SWITCH CIRCUIT}

The facility antenna switch circuit determines the strongest of the four $\mathrm{rf}$ receive signals from the cells. The board then passes this signal to the facility receiver board. A block diagram of the facility antenna switch circuit is shown in Fig. 6.3.

The if inputs to the board come from each of the facility transmit/receive circuits over a length of coaxial cable. Radio frequency circuitry on the board is identical for each of the four signal paths. Tracing the signal through one of the paths, the board first filters the rf input with a bandpass of 902 to $928 \mathrm{MHz}$ to remove out-of-band signals and interference. The signal is split with one path routed to an $\mathrm{rf}$ switch and the other to an if amplifier. The amplified signal is processed through an if amplitude detector that provides a voltage output proportional to the if power level.

The output of each detector is buffered and compared to the level of the other three channels through a matrix of voltage comparators. The state of each comparator is processed through digital control logic to activate the appropriate electronic rf switches to route the strongest signal to the facility receiver. The control logic allows voting among all inputs, not just among adjacent cell antennas. Therefore, proper receive antenna selection is possible when operating in a degraded mode with one facility antenna or transmit/receive module inoperative.

The control logic performs an additional function that is not obvious from the block diagram. The amplitudes from the if detectors are constantly changing, and the comparators switch to indicate the highest level signal. The control logic must inhibit the rf switches from changing during the transmission of a data packet if digital errors are to be avoided. The inhibit function is implemented by clocking a latch from the rising edge of the first carrier signal that occurs after the local transmitter shuts off. In operation, a logic signal resets the latch when the local system is switched into the receive mode. The level from each if detector is compared against a threshold that is set above normal background noise. When the cart transmitter turns on, the detector output on the facility antenna switch exceeds the preset threshold and the status of the antenna-to-antenna comparators is locked into the system. The rf switches activate the antenna that receives the strongest signal at the beginning of the transmission. The switches stay in this position until the cart transmission has ended, avoiding the switching of antennas during the middle of a data packet. If the active antenna drops into a signal null, the system will switch to a stronger antenna at the beginning of the next receive cycle.

A schematic diagram for the facility antenna switch circuit is given in Dwg. Q-6340-180. The number of parts dictated that the circuit be implemented on two printed circuit boards. The if circuits, level detectors, buffers, and rf switches are found on board Dwg. Q-6340-181A, and the comparators, voting logic, and inhibit circuits are on board Dwg. Q-6340-181B. Both circuit boards are housed in a card cage in the engineer's console.

\subsection{FACILITY AND CART RECEIVER CIRCUITS}

The rf receiver circuit converts the $915-\mathrm{MHz}$ FM signal to a baseband representation of the biphase digital data signal. The same circuit design is used for both the facility and the cart receivers. Figure 6.4 shows a block diagram of the receiver.

The receiver board follows the antenna control board in both the facility and cart systems. The 915-MHz rf signal feeds into an automatic gain control (AGC) block that maintains an optimum signal level for the receiver even though the if fluctuates due to both multipath and distance between transmitting and receiving antennas. The AGC consists of an electronic attenuator followed by an if amplifier that provides $20 \mathrm{~dB}$ of dynamic range control. The attenuator and amplifier stages are then repeated, bringing the total AGC range to $40 \mathrm{~dB}$. The output of the second rf amplifier is split and sampled by an rf amplitude detector that provides a voltage level 


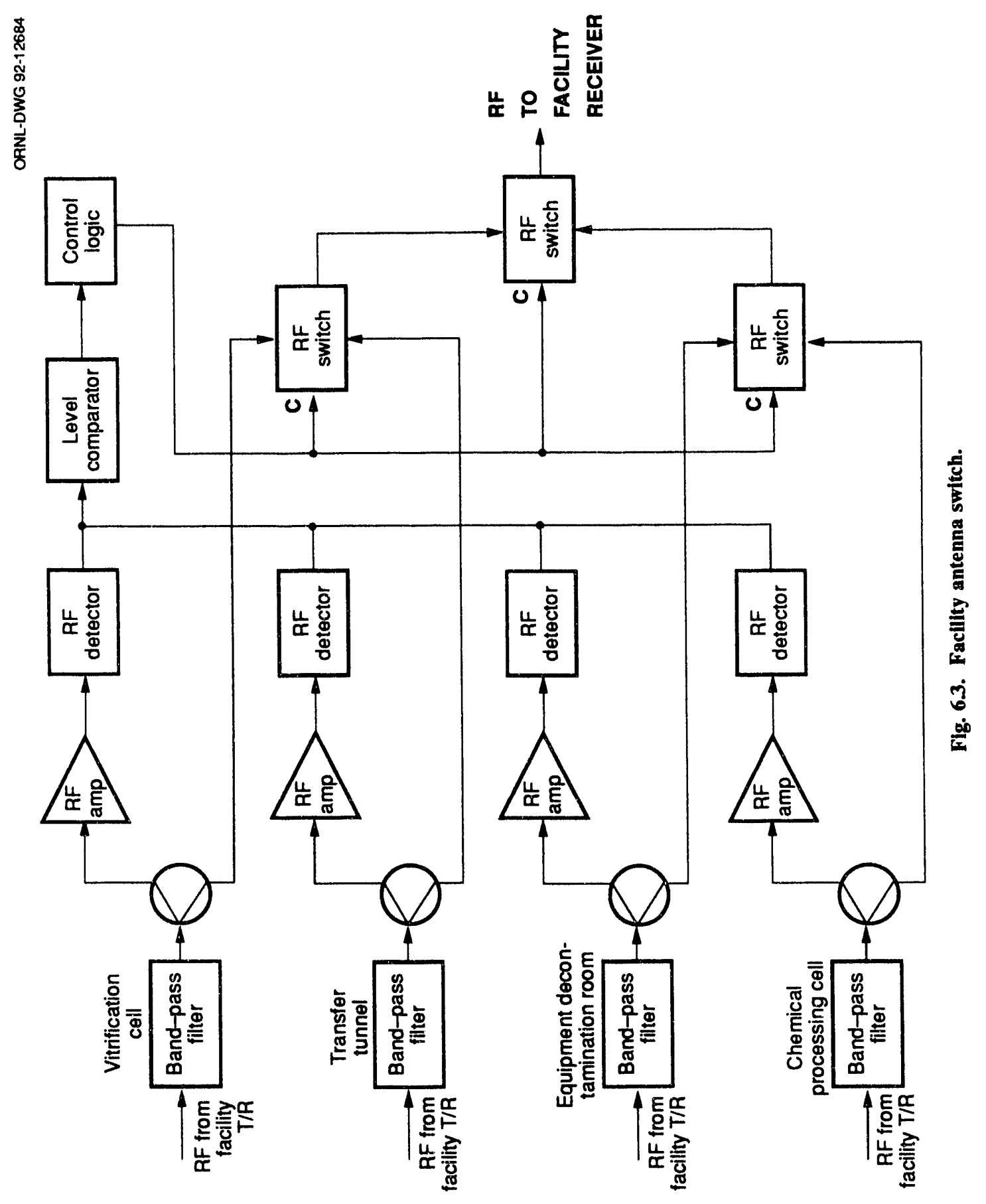




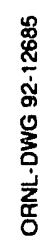

동
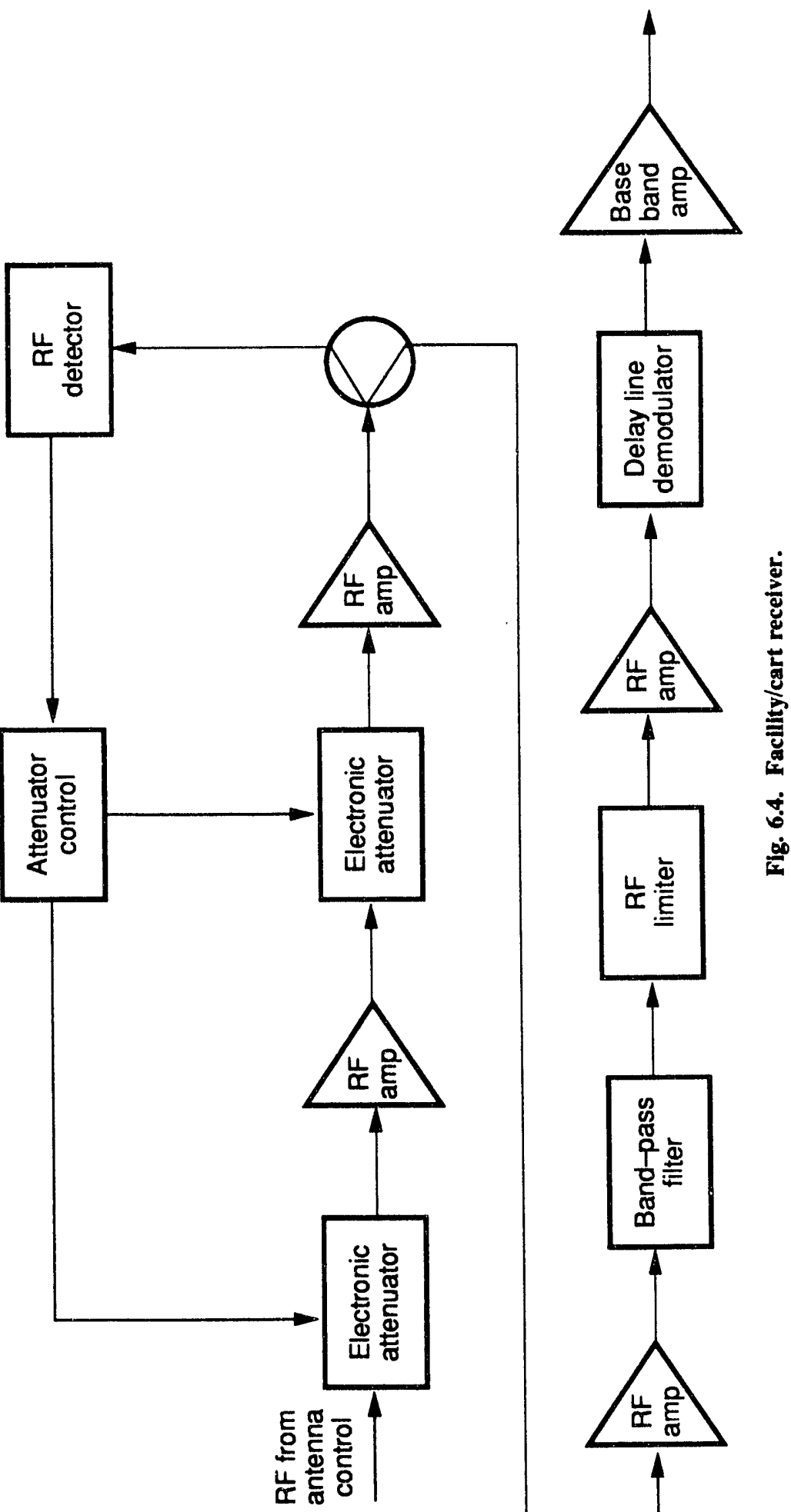

崖
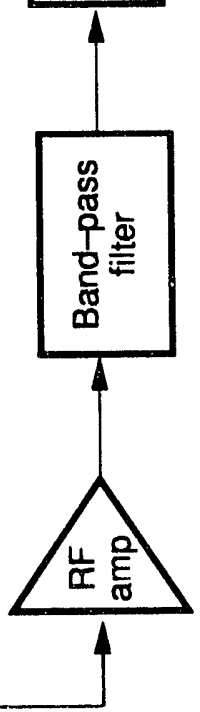
proportional to the if level. This voltage is amplified and applied through a feedback loop to control the electronic attenuators.

The output from the AGC is further amplified before being applied to a bandpass filter that removes any rf energy that is not within the $902-$ to $928-\mathrm{MHz}$ band. The signal is then applied to an rf limiter that removes fast amplitude fluctuations which would be detected as incidental AM on the signal. The output of the limiter is further amplified before being applied to the demodulator.

The delay line demodulator consists of a signal splitter, a coaxial delay line, and a balanced mixer. The signal is essentially multiplied with a delayed version of itself to produce a voltage that is proportional to the frequency shift of the rf signal. The output is low-pass filtered to remove the residual carrier and squared terms of the mixing process before being amplified by a baseband amplifier. The amplifier output is sent to the communications biphase encoder/decoder board for conversion to a true digital signal.

A schematic diagram for the facility/cart receiver is given in Dwg. Q-6340-260. The circuit board for the facility receiver is housed in a card cage in the engineer's console computer, while the circuit board for the cart receiver is housed in a card cage inside the lead shielded enclosure on the cart.

\subsection{CART ANTENNA CONTROL CIRCUIT}

The cart signal comparison circuit, also called the cart antenna control, receives rf signals from the two cart antennas, selects the stronger signal, and passes it to the receiver circuit. A block diagram of the circuit is shown in Fig. 6.5. This circuit combines the functions of antenna transmit/receive control and receive antenna selection. When the transmit/receive control line is in the transmit position, the lower rf switch connects the transmitter output to Antenna 2 . The rf switches remove the input signals from both rf receiver amplifiers when in the transmit mode. Because the two cart antennas are located in close proximity to one another, transmitting from both would produce a directional pattem that could degrade the signal received by the facility antennas. Additionally, there is no way of determining the best transmitting antenna as the cart moves through signal nulls. Furthermore, the cart-to-facility data are not considered as critical as the facility-to-cart data, and a single transmitting antenna is considered sufficient.

In the receive mode, the $\mathrm{rf}$ from each antenna is routed through the $\mathrm{rf}$ switches to the respective low-noise $\mathrm{rf}$ amplifiers and the if circuitry on the board becomes identical for the two signal paths. Each signal is amplified by $40 \mathrm{~dB}$ and passed through a filter with a bandpass of 902 to $928 \mathrm{MHz}$ to remove out-of-band signals and interference. The signal is split with one path routed to an $\mathrm{rf}$ switch and the other to an rf amplifier. The amplified signal is processed through an rf amplitude detector that provides a voltage output proportional to the $\mathrm{rf}$ power level.

The output of each detector is compared against the level of the other signal. The comparator determines the position of the electronic rf switch that routes the stronger signal to the cart receiver. The control block performs the additional function of locking the rf switch once the transmission begins. Because the amplitudes from the rf detectors are constantly changing, the comparator will switch to the higher-level signal. The control logic must inhibit the rf switch from changing during data packet transmission to avoid data errors. The inhibit function is implemented by clocking a latch from the rising edge of the first carrier signal that occurs after the local transmitter shuts off. In operation, a logic signal resets the latch when the local system is switched into the receive mode. The level from each rf detector is compared against a threshold that is set above the normal background noise level. When the facility transmitter tums on, the detector output on the cart antenna switch exceeds the preset threshold and the status of the antenna comparator is locked into the system. The rf switch activates the antenna that receives the strongest signal at the beginning of the transmission. The switch stays in this position until the 


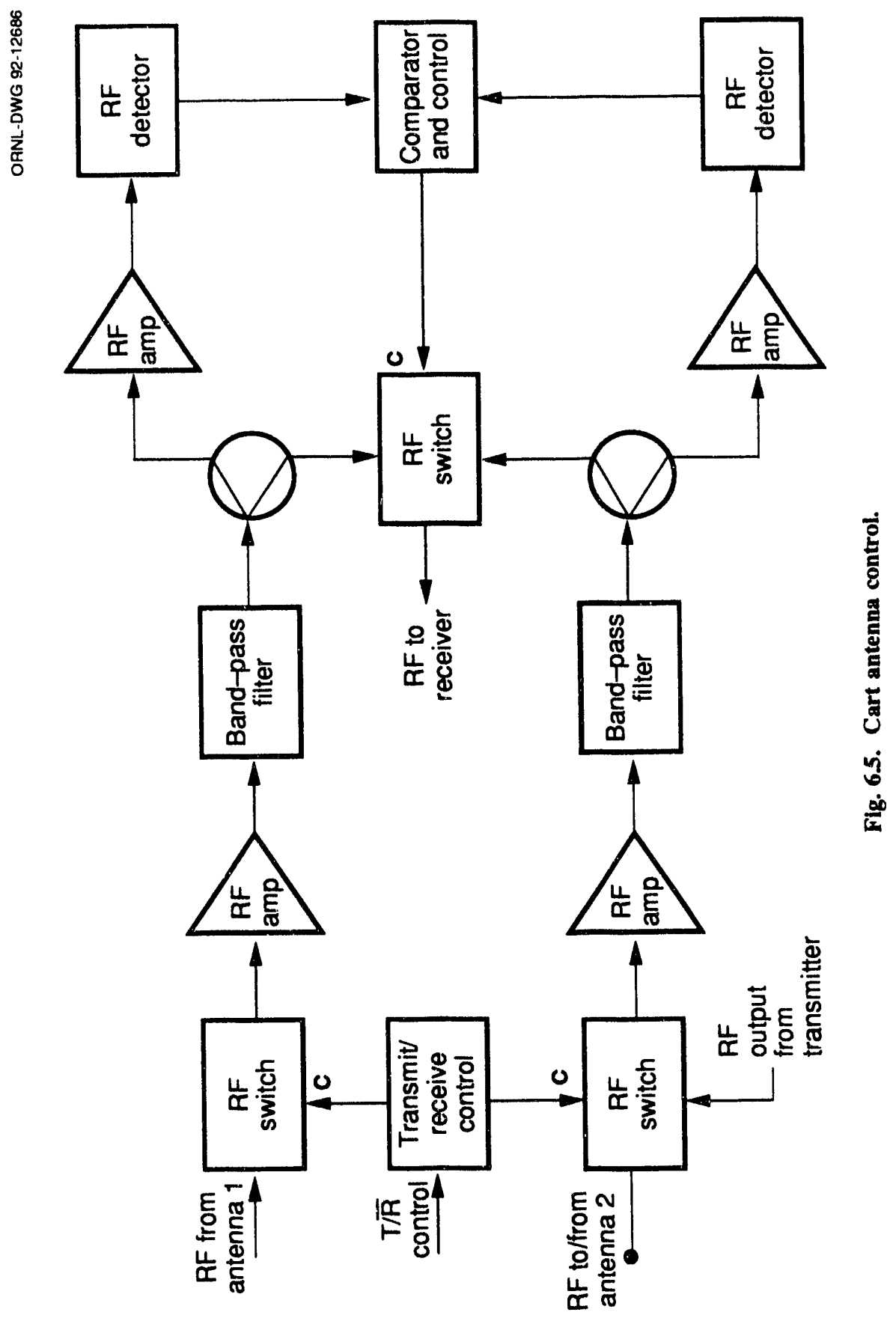


facility transmission has ended, avoiding the switching of cart antennas for the duration of the data packet. If the active antenna drops into a signal null, the system will switch to the stronger antenna at the beginning of the next receive cycle (a maximum of $\sim 1-s$ duration).

A schematic diagram for the cart antenna control circuit is given in Dwg. Q-6340-265. The circuit board is housed in a card cage inside the lead shielded enclosure on the cart.

\subsection{CART TRANSMITTER CIRCUIT}

The cart transmitter circuit converts the digital data from the cart microprocessor to an FM rf carrier that is suitable for transmission to the facility cell antennas. A block diagram of the cart transmitter is shown in Fig. 6.6. The circuit acquires a digital message packet from the communications biphase encoder/decoder board. The biphase signal is processed through a modulation amplifier that allows adjustment of both the rf carrier frequency and the deviation of the FM signal. The modulation amplifier drives a VCO with a carrier frequency of $915 \mathrm{MHz}$ in the ISM band. Deviation of the carrier is adjusted to $\pm 10 \mathrm{MHz}$ around the carrier.

The VCO output is buffered through an attenuator and rf amplifier to isolate the oscillator from load variations that could pull the frequency. The rf signal is then amplified by a power amplifier to generate a level of $+17 \mathrm{dBm}(50 \mathrm{~mW})$ of $\mathrm{rf}$ power. The if output from the transmitter board is routed through the cart antenna control board to the cart antenna. A transmit/receive control signal is supplied by the cart communications microprocessor to activate the VCO and rf amplifiers during the transmit mode and deactivate them during the receive mode.

A schematic diagram for the cart transmitter is given in Dwg. Q-6340-270. The circuit board is housed in a card cage inside the lead shielded enclosure on the cart.

\subsection{BIPHASE ENCODER/DECODER}

The biphase encoder/decoder for the communications link for the transfer cart is designed to convert 8-bit parallel data into a biphase-encoded serial stream for transmission across an rf link and then decode the serial biphase back into 8-bit parallel data at the other end of the link. Two versions of the encoder/decoder board were designed: one for the facility end of the communications link and one for the cart end of the link. The two boards are almost identical; the differences are limited mainly to those circuits and functions that were required to accommodate the different needs of the facility and cart computers. A schematic diagram for the facility-side board is shown in Dwg. Q-6340-185, and the cart-side board is shown in Dwg. Q-6340-290.

The encoder/decoder uses the biphase- $L$ encoding format for the serial data stream. Biphase was selected because of its widespread use and because there are several devices on the market that contain the encoding and decoding circuits on a single chip. Of these circuits, the DP8342/8343 encoding/decoding pair, from National Semiconductor, was selected because it has been used previously in radiation-tolerant designs and has repeatedly demonstrated gamma tolerance in excess of $10^{7} \mathrm{rad}$. Its baud rate can be set for any rate up to $3.5 \mathrm{Mbaud}$, but for this instance a rate of $125 \mathrm{kbaud}$ has been set as a good tradeoff between data rate and bandwidth.

The encoder/decoder transmission format is of the packet typc, in which the data is sent in coherent bursts rather than in a continuous fashion. The packet format lends itself to bidirectional communications and to digital control systems since it facilitates hand-shaking routines. It also is essential for transmitting data at a baud rate that is significantly higher than the $1 / O$ throughput of the sending/receiving device, which for the transfer cart is an STD-based microprocessor. Since the burst-type transmission requires that all of the data be immediately available for transmission, the entire packet must be stored in the input stage of the encoder and likewise in the output stage of the decoder. The size of the input/output storage circuits limits the packet size for this system to 48 bytes. There is also a provision for a special two-byte packet that will cause the decoder to 


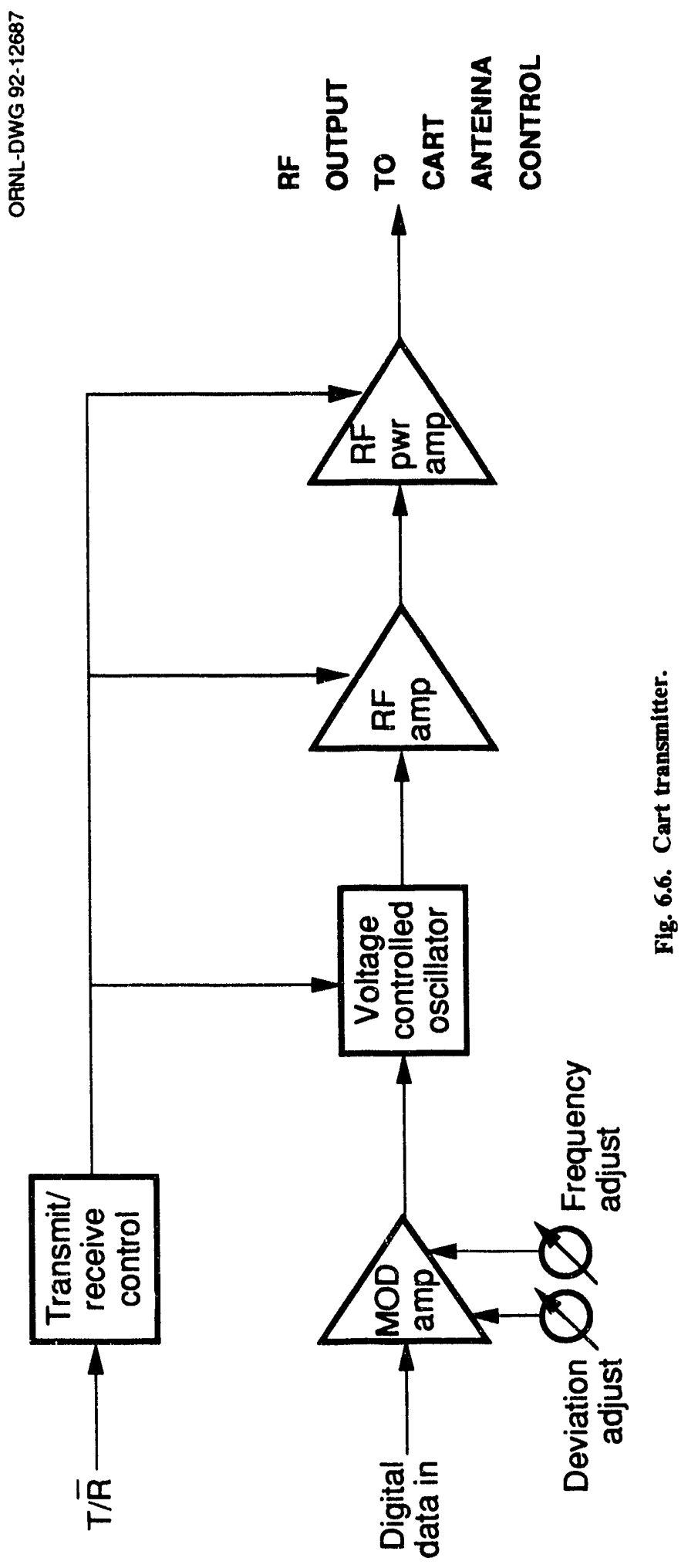


generate a reset pulse that can be used to remotely reset the equipment on the decoder end of the link. The reset will also reinitialize the decoder circuitry. This remote reset function is implemented only on the cart version of the encoder/decoder board; it is not a desirable feature on the facility version of the board.

The encoder/decoder is designed to interface directly to a microprocessor circuit or computer via three 8-bit ports such as in the 8255-type interface chip or an 8255-based 1/O card. It is divided into two major functional blocks: the biphase encoder and the biphase decoder.

\subsubsection{Interface Protocol}

The facility encoder/decoder requires five control signals from the facility communications controller computer (BYTE1, SI, /TX, RST, and /RD) and eight bits of bidirectional data. It generates five status signals (DATRDY, OR1, ERRFLG, FSTBT, and IR) for use by the facility communications controller computer.

The cart encoder/decoder requires five control signals (BYTE1, SI, TX, RST, and RD) and eight bits of bidirectional data. It generates five status signals (ERRFLG, OR1, IR, FSTBT, and DATRDY). The T/R control line and the ANTSTAT status line feed directly to the link hardware and do not affect the encoder/decoder.

For transmission of data, four control steps are required. The first step is to test the status signal IR, which is derived from the input stage of the encoder. A logic high on this line indicates that the input stage is ready to accept data.

The second step is to write the first byte of data into the encoder. This step is treated separately from the rest of the data because a flag bit, called BYTE1, must be written with the first byte. The data is asserted and BYTE1 is driven high at the same time, and both are then clocked into the input stage by a positive-going pulse on the facility board's SI control line or a negative-going pulse on the cart board's SI control line. The data is then deasserted, and BYTE1 is driven low. BYTE1 remains low for the rest of the packet, being used only for marking the very first data byte.

The third step is to write the remaining bytes of data into the encoder, which is done in the same manner as writing the first byte except that BYTE1 stays low. Each time a new byte is to be written, IR should be checked to make sure it is high. A low indicates that the input stage is not ready to accept data, either because of a malfunction in the encoder or because the packet is too large to fit in the input storage section of the encoder.

The fourth step is to assert TX low (high for the cart version) after the last data byte has been entered into the encoder. This will initiate the encoder and transmit the data. TX must be returned to the deasserted state before the next packet is sent.

The encoder encodes the data two bytes ( 16 bits) at a time, adding one byte of the cyclic redundancy check (CRC) code to every two bytes of data. If an odd number of bytes is entered, the encoder will append a null byte to the last byte to make a full pair. Thus if four bytes of data are entered, the encoder will divide the four data bytes into two pairs and add a CRC byte to each pair, transmitting six bytes of information. Three bytes of data will be divided into two pairs, the first being the first two data bytes and the second being the third byte plus a null byte. The total number of bytes transmitted will also be six bytes. The CRC byte will be stripped off in the decoder, but the null byte will be appended to the data.

To generate the remote reset pulse, only two bytes of data need be sent. Both bytes must match the reset code set in the decoder circuit. Since the reset generator searches for an 8-bit data pattern in both bytes in a data byte pair, it is possible for random data to trigger a reset pulse if steps are not taken to prevent both bytes in any given data byte pair from matching the reset code. Two identical bytes that match the reset code and are consecutive but are not paired by the encoder will not trigger a reset. 
A high on DATRDY indicates that data have been received by the decoder and are waiting to be read. OR1 should also be high and indicates that the output buffers [first-in, first-out (FIFOs)] contain data. Driving /RD low (high for the cart version) will assert the data onto the data bus (D0-D7), and its rising (falling) edge will cause the next available byte to be present in the output stage of the decoder. DATRDY will also be reset to the low condition with the first transition on /RD and will remain low until a new packet of data is decoded. The OR1 signal will go low with the /RD signal, so OR1 must be checked for a high level before the byte is read. OR1 will continue to retum to the high state until the last byte has been read.

A high on the ERRFLG signal indicates an error in the current or previous byte. This signal will only be valid for even-numbered bytes, which are the second bytes in the encoded pairs. Thus when reading the even-numbered bytes, this signal must be checked to make sure that the data is valid. If it is high, the byte being read and the byte that came before it should be discarded unless an error-correction routine is being implemented in the host microprocessor.

The RST signal should be asserted high to reset the encoder/decoder. A power-up reset is advisable, as is a reset before writing each packet to the encoder.

\subsubsection{Biphase Encoder}

The following circuit descriptions are for the facility .version of the encoder/decoder. Component numbers for the cart version are in brackets (reference Dwgs. Q-6340-185 and Q-6340-290).

The biphase encoder block appears in the top half of the encoder/decoder schematics. Its major functional sections are (1) the input FIFO, composed of U2, U4, U6A, and U6B [U14, U15, U23A, and U23B]; (2) the parallel-to-serial conversion stage, U5 [U16]; (3) the CRC generator, U16 [U25]; (4) the control section, composed of U9, U10, and U17 [U21, U22, U32, and U34A]; (5) the output FIFO stage, composed of U18, U19, and U20 [U1, U2, and U9]; (6) the biphase encoding stage, composed of U1 and U8 [U3 and U8]; and (7) the timebase section, composed of U14C, U27, and U31 [U28, U29C, and U33].

\subsubsection{Input FIFO stage}

The input FIFO stage of the biphase encoder block serves three functions. First, it provides temporary storage of the 8-bit parallel data to minimize problems with converting an irregular parallel flow into a regular serial flow. Second, it serves as a synchronizing buffer between two digital systems which are, potentially, at very different clock rates. Third, it allows the transmission data rate to vary greatly from the rate at which the parallel data is written to the encoder. The FIFO itself is composed of U2 and U4 [U14 and U15], which are C67402 $64 \times 5$ asynchronous FIFOs. Like the DP8342/8343 encoder/decoder pair, the C67402 has been used extensively in radiation-tolerant designs and has repeatedly operated normally beyond $10^{7}$ rad. U6A [U23A] performs a logical AND on the input ready (IR) status outputs from the two FIFOs, and U6B [U23B] performs a logical AND on the output ready (OR) signals for use by U9 [U22].

\subsubsection{Parallel-to-serial converter}

The parallel-to-serial converter consists of U5 [U16], which is a 74LS166 parallel-load shift register. It is under the control of U9 [U21], which toggles the parallel-load enable input every 8 clock cycles for the two data bytes in the pair while the encoder is transmitting. When the CRC byte is ready to be inserted into the serial stream, the parallel-load signal does not toggle, which then causes the data to be all zeroes. 


\subsubsection{CRC generator}

The CRC generator is U16 [U25], a 9401/8X01 CRC generator/checker chip. Its output, the CRC byte for each data byte pair, is generated from the serial output of U5 [U16] and is clocked out when its CWE input is driven low. The output is fed to U9 [21] in the control section. Detailed timing diagram appears in Fig. 6.7.

\subsubsection{Control section}

The control section consisis of U9, U10, and U17 [U21, U22, U32, and U34A]. U9 [U21] is the heart of the control section and generates most of the signals that cause the encoder to operate. It monitors the input FIFO stage and the output of U17 [U32] and uses the signals from these sections to pair the data bytes, generate and insert the CRC byte, and load the composite stream into the output FIFO stage for transmission. U10 [U22] is used primarily as a modulo-24 counter. Its count output is fed to U9 [U21], which uses it to monitor its location in the encoding process. U9 [U21], in tum, enables and disables the counter in U10 [U22]. U17 [U32] is a latch that guarantees that the /TX signal will last for a minimum of two clock transitions so that U9 [U21] will always be able to see it.

When a data packet is first written to the encoder by the computer, the OR outputs of the input FIFOs will go high, as will the B1 signal, which is the BYTE1 flag marking the first byte in the packet. If the B1 flag does not go high along with the OR signal, U9 [U21] will clock out data bytes from the FIFO until B1 and OR are both high or until the FIFO is emptied. The STRT signal from $\mathrm{U} 17$ [U32] is used as the trigger to initiate the encoding cycle. A negative [positive] pulse on the /TX control line will set U17 [U32], causing STRT to rise for at least two clock edges. The high on all three signals starts U9 [U21], which, in turn, starts U10 [U22]. As U10 [U22] counts from 0 to 24, U9 [U21] decodes the count and from it generates the encoder control signals. The detailed timing can be found in Fig. 6.7. At the count of 24, U9 [U21] checks its status lines from the input FIFO stage and will either continue enc ding (if more data resides in the FIFO) or quit (if the data is gone). U9 [U21] also inserts the CRC code into the data by enabling the CRC generator and switching the CRC output into the serial stream.

\subsubsection{Output FIFO stage}

The serial data/CRC stream from U9 [U21] is fed to U20 [U1], the serial-to-paralle' converter in the output FIFO stage. U20 [U1] is an 8-bit, serial-in, parallel-out shift register whose parallel output goes to the input pins of U18 and U19 [U2 and U9], which make up the FIFO. U9 [U21] then generates a shift-in pulse at 8-bit intervals to clock the output of U20 [U1] into U18 and U19 [U2 and U9].

\subsubsection{Biphase encoder stage}

The output of U18 and U19 [U2 and U9] are then fed to U8 [U3], which is the DP8342 biphase encoder chip. U1 [U8] controls the transfer of data from the FIFO to the encoder. U8 [U3] adds a synchronization bit and a parity bit into the serial stream during encoding for a total of 10 bits per 8-bit byte, so the data will be read out of the FIFOs at a lower rate than it is being written in by $\mathrm{U} 9$ [U21].

Thus for this stage there is no reason to store the entire packet in the FIFO; U1 [U8] starts transferring data from the FIFO into the encoder as soon as it reaches the outputs of the FIFO. U8 [U3] disables the biphase decoder (U24 [U10]) during transmission by raising its transmitteractive output. 


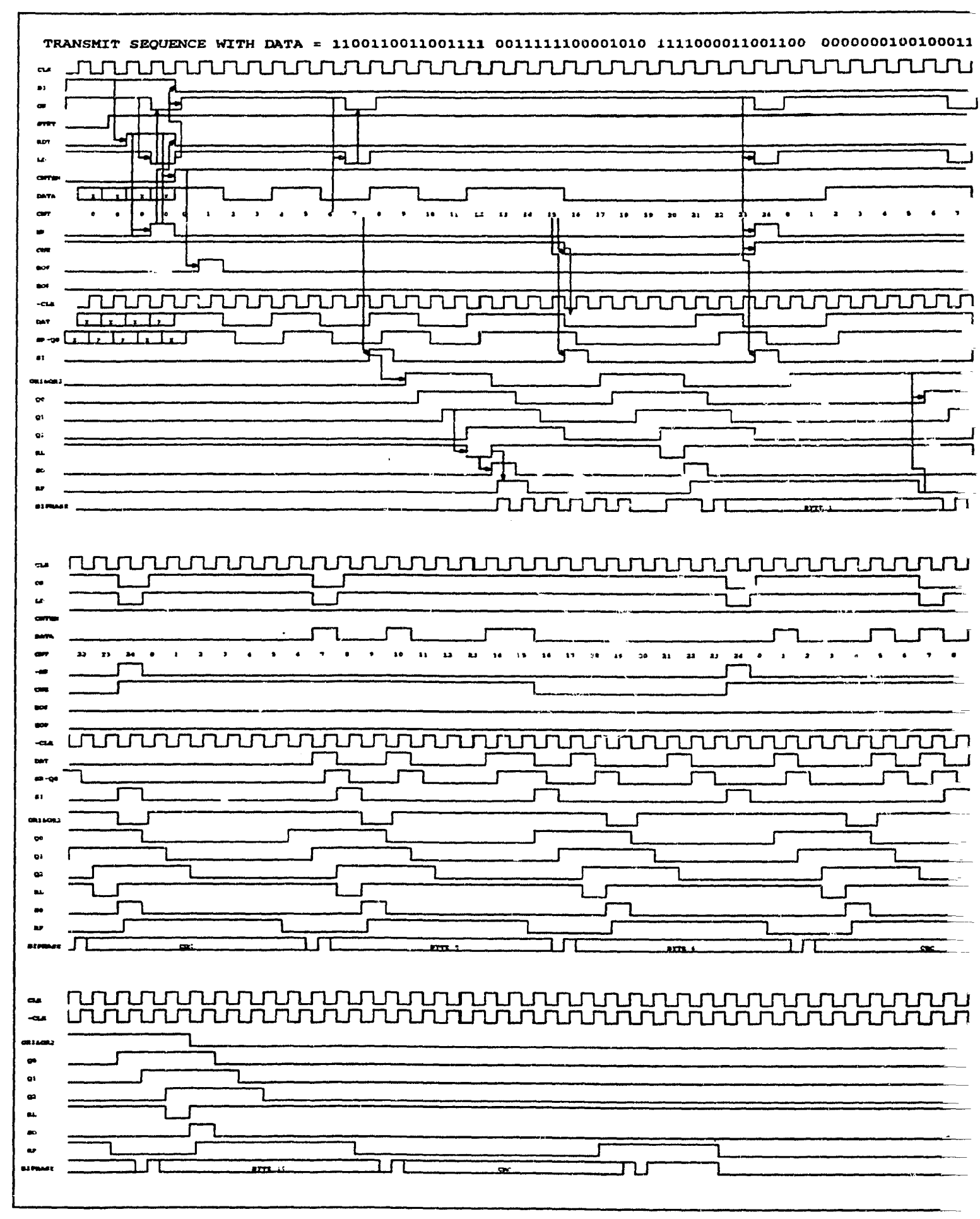

Fig. 6.7. Biphase encoder/CRC gene 
100010101100111

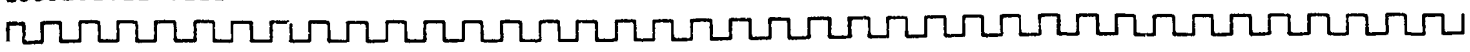

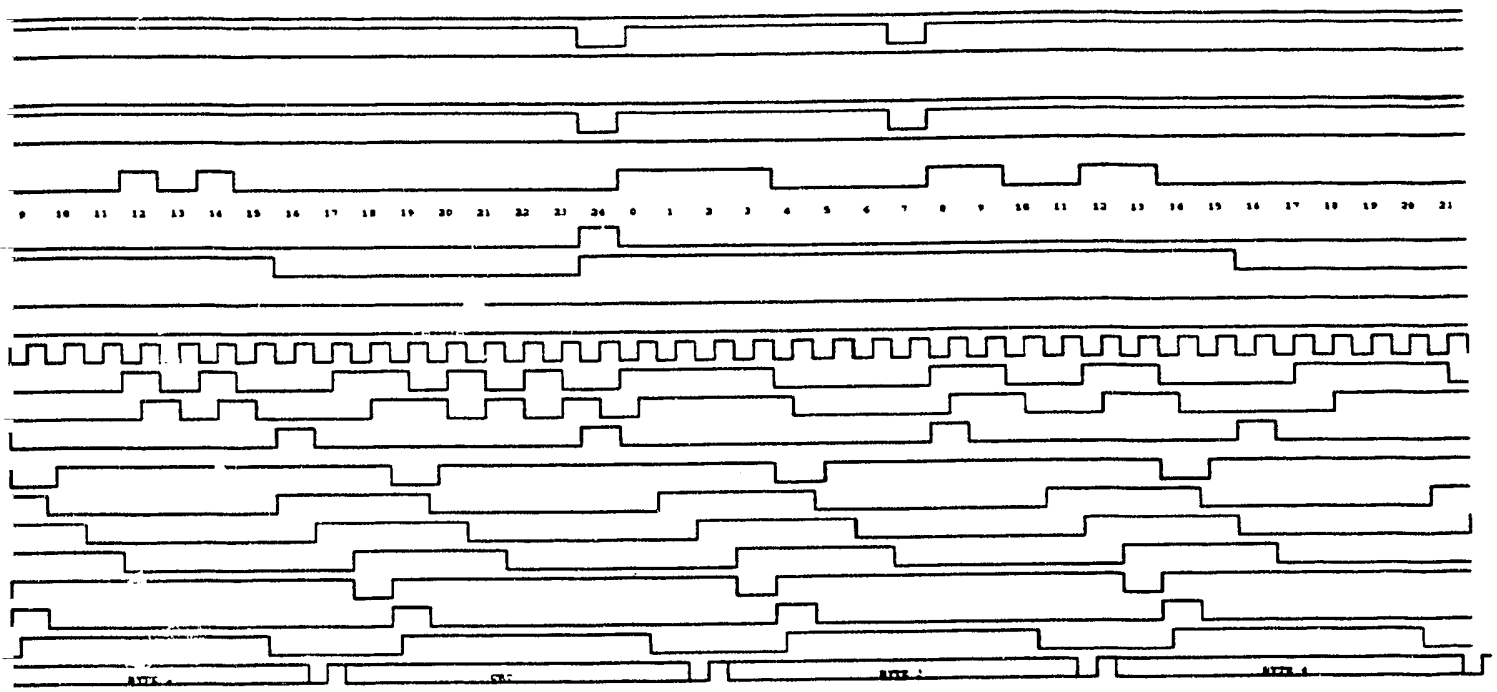

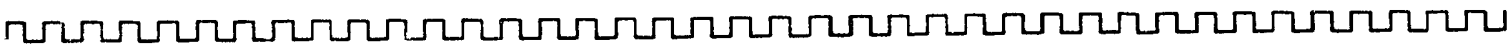
(2) 10"

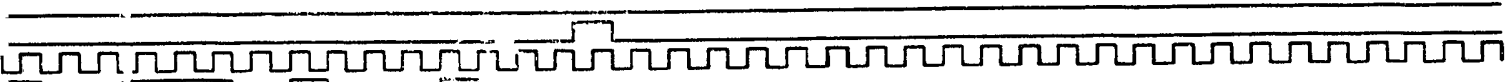

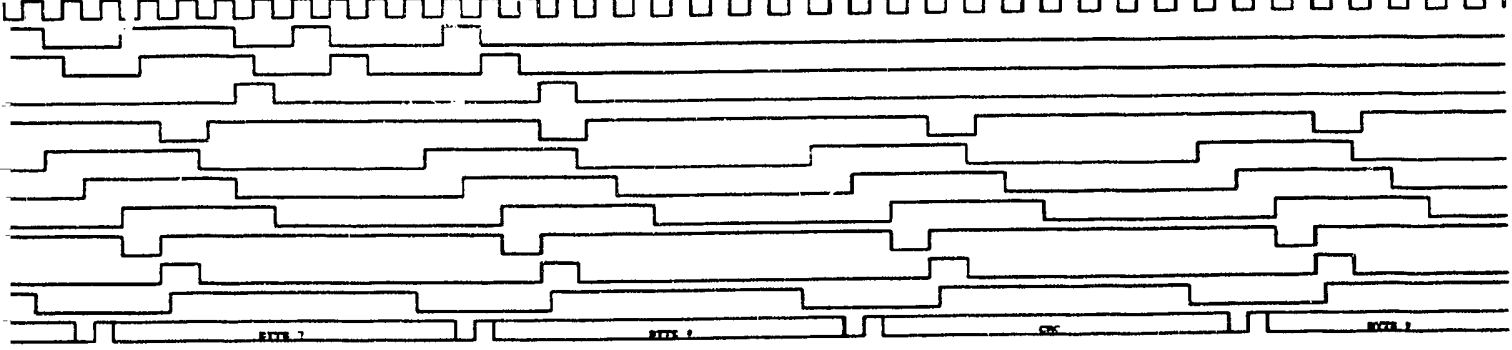

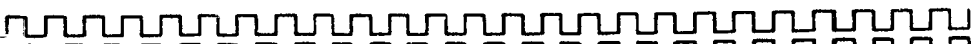

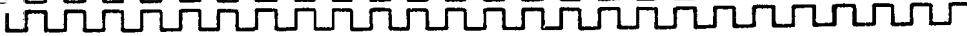

WUNS CART

BIPHASE RACODER / CRC gENERATOR

TIMCONG DIAGRAM

WITH DATA VALUES 


\subsubsection{Timebase section}

The timebase section, U27, U31, and U14C [U28, U33, and U29C], generates the two clock waveforms that are needed to operate the encoder/decoder circuit. U27 [U28] is a dual in-line package oscillator running at a frequency that is 16 times the serial transmission bit rate. In this case U27 [U28] is $2.0 \mathrm{MHz}$. U31 [U33] divides the output of U27 [U28] by 2 and by 16 . The divide-by-2 output is used by U8 [U3] in the encoder and by U24 [U10] in the decoder. The divide-by-16 output is buffered by U14C [U29C] and is used to drive all of the other clocked circuits with the exception of U20 [U1] in the encoder and U13 [U24] in the decoder. These two chips must be on the alternate clock phase because the CRC generator chips U16 and U21 [U25 and U30] are falling-edge triggered and U20 [U1] and U13 [U24] must be in phase with them.

\subsubsection{Biphase Decoder}

The biphase decoder block appears in the lower half of the encoder/decoder schematic, and its detailed timing diagram appears in Fig. 6.8. Its major functional sections are (1) the input comparator, U32 [U6]; (2) the DP8343 biphase decoder chip, U24 [U10]; (3) the input storage FIFO and associated circuits, composed of U15, U23, U25, and U30 [U5, U11, U12, and U20]; (4) the parallel-to-serial converter, U22 [U19]; (5) the control section, composed of U28 and U29 [U26 and U27]; (6) the CRC checker, U21 [U30]; (7) the serial-to-parallel converter, made of U12 and U13 [U17 and U24]; (8) the output storage section, composed of U3, U7, U11, U6C, U26A, U14E, U14F, and U26B] [U4, U7, U13, U23C, U29E, U31A, U31B, U34A, and U34B]; and (9) the remote reset comparator U18 (cart version only).

\subsubsection{Input comparator}

The input comparator stage converts the incoming biphase waveform from the rf receiver into a TTL (transistor-transistor level) signal. The comparator is an LM161 high-speed TTL-output comparator. The biphase signal is terminated with a $4.7-\mathrm{k} \Omega$ resistor and applied to the noninverting input of the comparator; the reference voltage, taken from the $10-\mathrm{k} \Omega$ trimmer $R 12$ [R4], is applied to the inverting input. The reference voltage ranges from about $+2 \mathrm{~V}$ to about $-2 \mathrm{~V}$. Since the differential input limit of the LM161 is about $5 \mathrm{~V}$, care should be taken to ensure that the difference between the biphase signal from the receiver and the reference voltage does not exceed $\pm 5 \mathrm{~V}$. Limiting the input to $\pm 3 \mathrm{~V}$ will ensure that this condition is met. The output of the comparator is a differential TTL signal taken off pins 9 and 11 of the comparator.

\subsubsection{Biphase decoder}

The biphase decoder chip, U24 [U10], converts the differential biphase signal from U32 [U6] into its constituent 8-bit bytes.

\subsubsection{Input storage FIFO}

The input storage FIFO is very similar to the other FIFO stages in the encoder/decoder. It is composed of two C67402 64x5 FIFOs arranged in parallel. U23 and U30 [U11 and U5] transfer the data from the biphase decoder chip to the FIFOs. U30 [U5] is used primarily as a pulse synchronizer/stretcher to ensure that the status flags from the decoder chip are of sufficient length and are timed correctly. In the case of the data available (DA) signal from the 8343, the purpose of U30 [U5] is more correctly described as ensuring that the period between active states of the signal is longer than 1 clock cycle, rather than ensuring that the signal's active states are long 


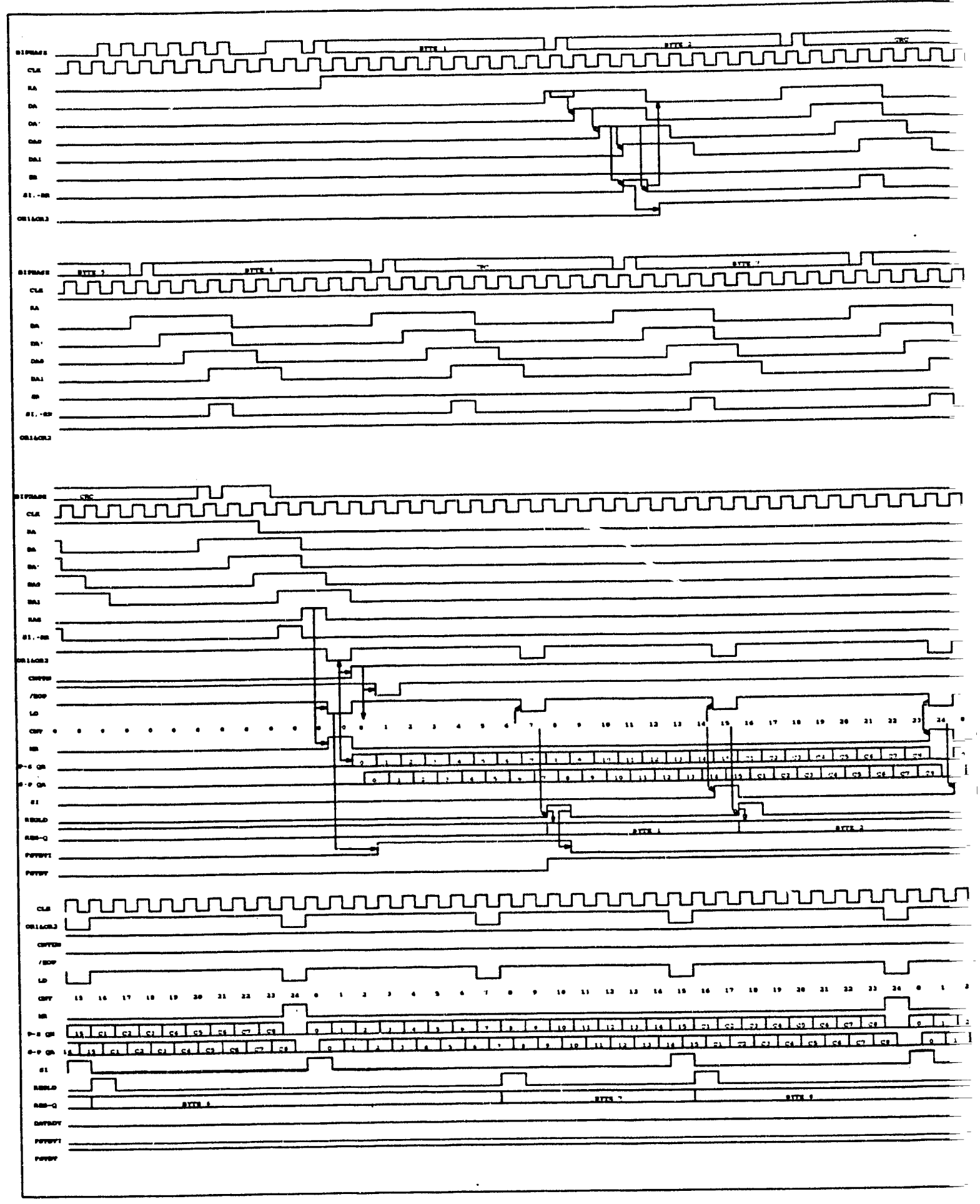

Fig. 6.8. Biphase encoder/CRC checke 


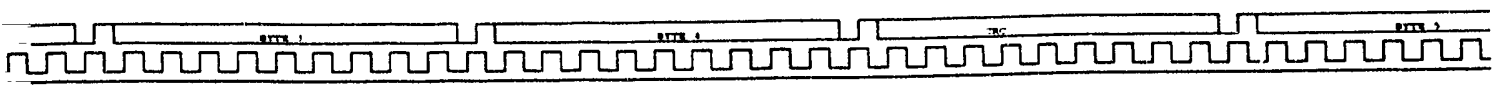

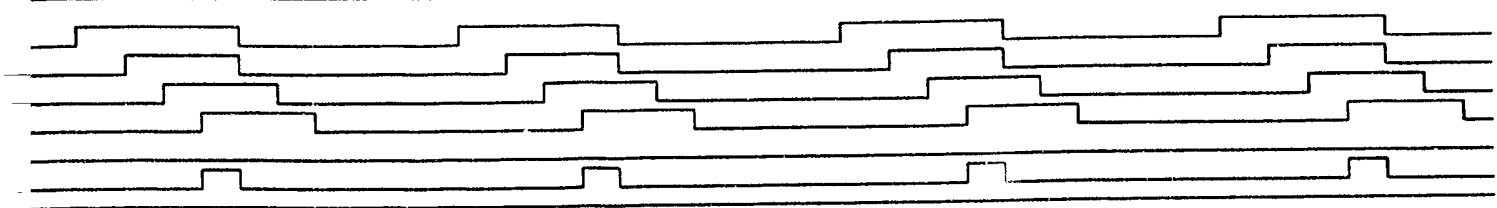

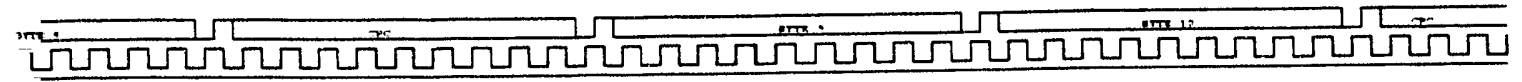
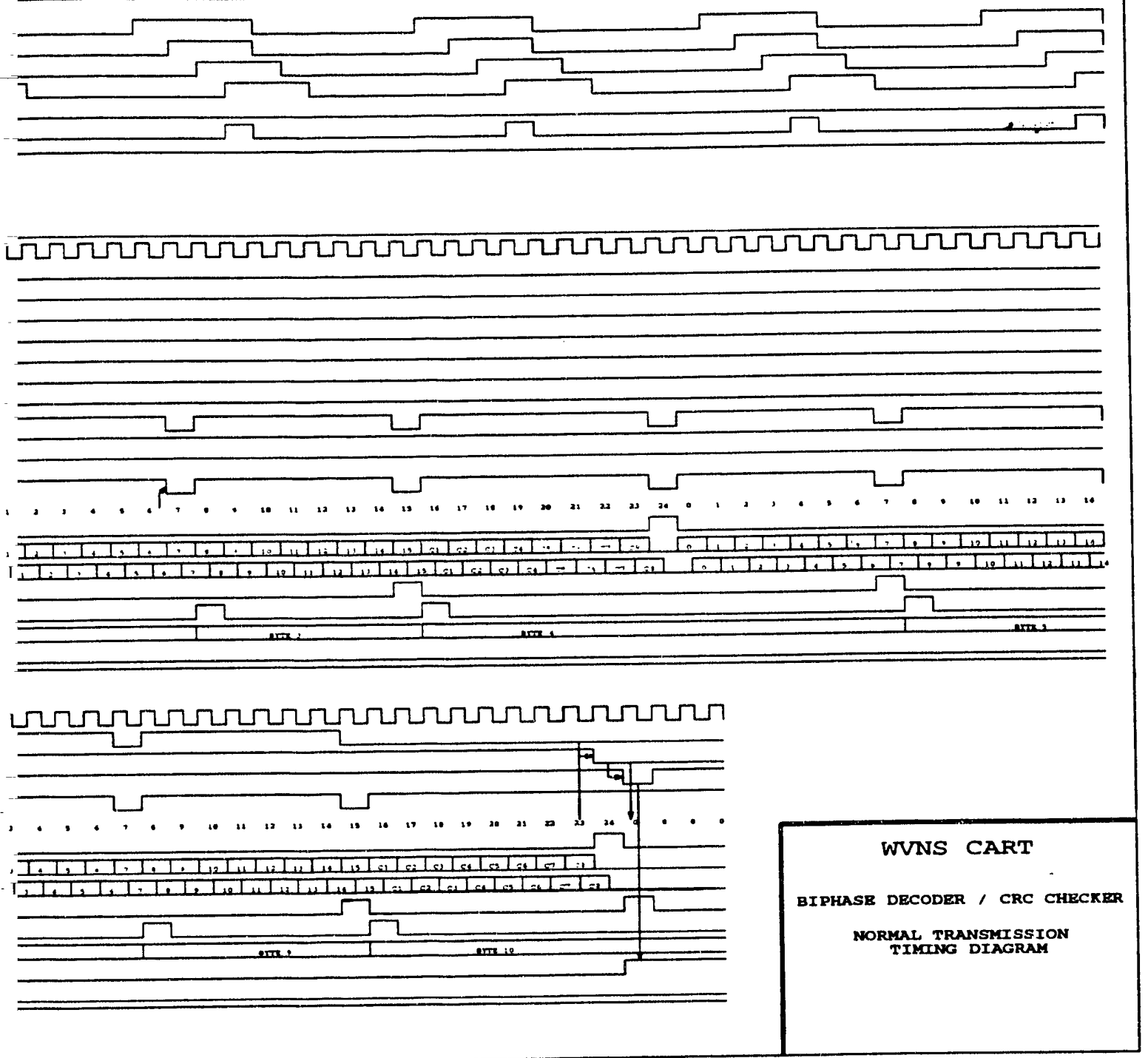

normal transmission timing diagram. 
enough, since there is one situation in which the period between two successive active states can be considerabiy less than 1 clock cycle of the system clock. U23 [U11] derives a register read (RR) pulse and a FIFO shift in (SI) pulse from the DA signal. These two signals conspire to transfer the incoming data bytes from the decoder to the FIFO. The RAE signal, which is used by the control section to initiate its sequence, is derived from the receiver active (RA) signal from the 8343. It rises for 1 clock cycle at the end of the data packet, triggered by the falling edge of the RA signal.

\subsubsection{Parallel-to-serial converter}

The parallel-to-serial converter, U2322 [U19], is a 74LS166 parallel-load shift register. The control section causes it to load every 8 clock cycles while the decoder block is active, using a single-cycle, low pulse on the load input. U14D [U29D] inverts the LD signal for use by the FIFO, causing it to shift out the next available byte on the falling edge.

\subsubsection{Control section}

The control section is composed of two programmable array logics (PALs), U29 [U27], which is the same counter PAL used in the encoder block, and U28 [U26], which is the primary control circuit in the decoder. The operation of the control section is similar to that of the encoder block's control section. The control sequence is initiated by the positive pulse on RAE from U23 [U11]. U28 [U26] then initiates U29 [U27] and decodes its count into a series of control pulses that first cause U22 [U19] to load every 8 clock cycles, then load the data into the temporary holding register U12 [U17] after it has been reconverted to parallel form by U13 [U24], then check the status of the error flag from the CRC checker at the appropriate time, and then write the data into the output FIFO until the end of the packet is reached.

An ancillary circuit to the control section is the first byte flag circuit, composed of U14F and U26B [U34C and U31B]. This circuit uses the beginning-of-packet (/BOP) and the end-of-packet (/EOP) signals from U29 [U27] to flag the first byte from each packet. The flag is written into the output FIFOs along with the first byte, and appears at their output as the FSTBT output status signal to the computer. These signals allow more than one packet to be stored in the output section at one time by separating the packets.

/BOP will pulse low for 1 clock cycle when a transmission is first received by the decoder, and /EOP will pulse low for 1 clock cycle at the end of the transmission. In the cart version /EOP is also made available to the antenna control printed circuit board in the link hardware via J3.

\subsubsection{CRC checker}

The CRC checker, U21 [U30], is a 9401/8X01 CRC generator/checker chip. This is the same IC that is used by the encoder to generate the CRC word, but in the decoder usage it is used to monitor the received data stream rather than insert a CRC word into the data. After each 16 bits of data plus their 8-bit checkword has been entered, U21 [U30] will generate an error indicator flag to signify whether or not an error was detected. U28 [U26] in the control section monitors this flag and takes appropriate action if an error was detected. Timing details are in shown in Figs. 6.8 and 6.9 . 


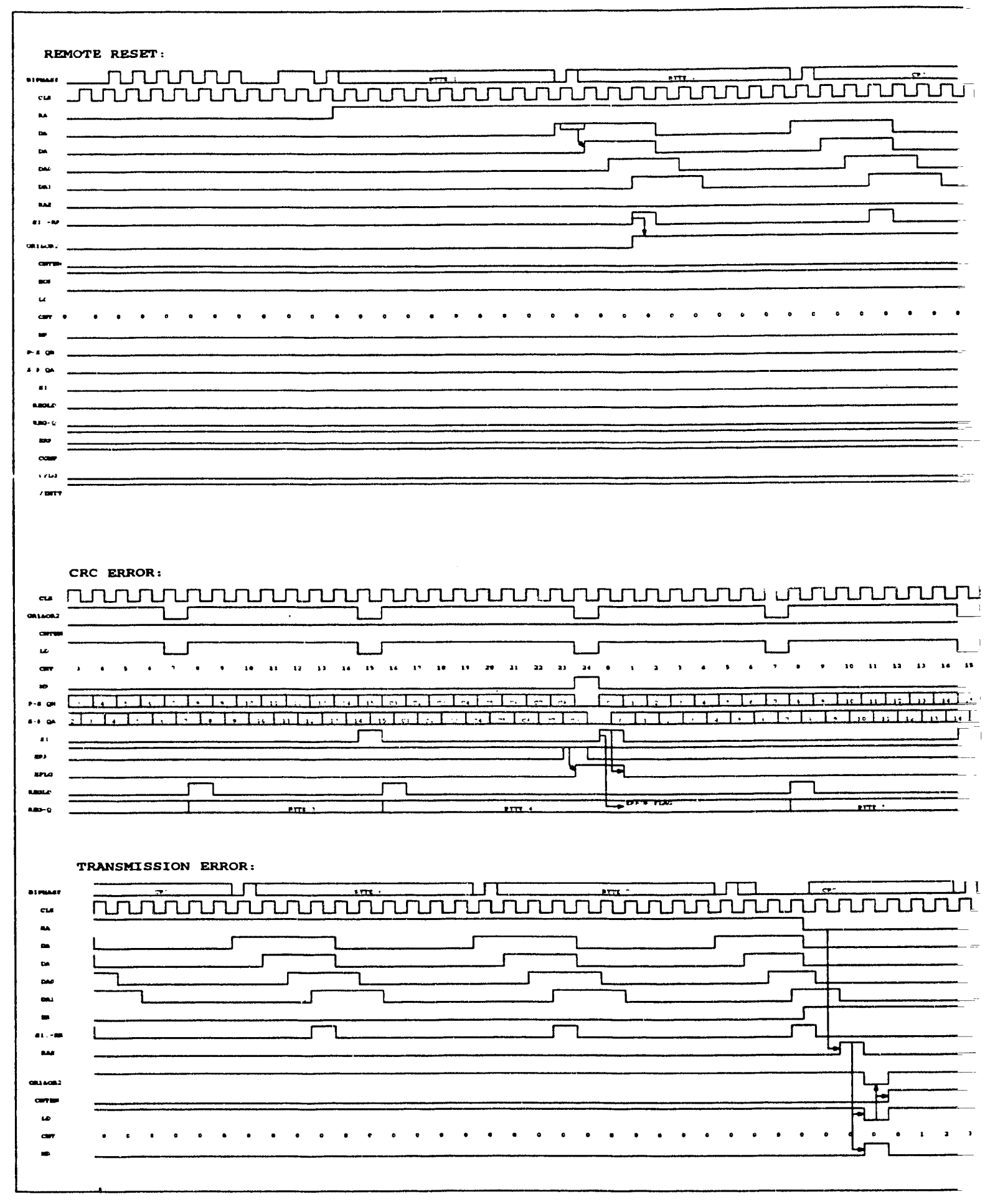

Fig. 6.9. Biphase decoder/CRC che: 

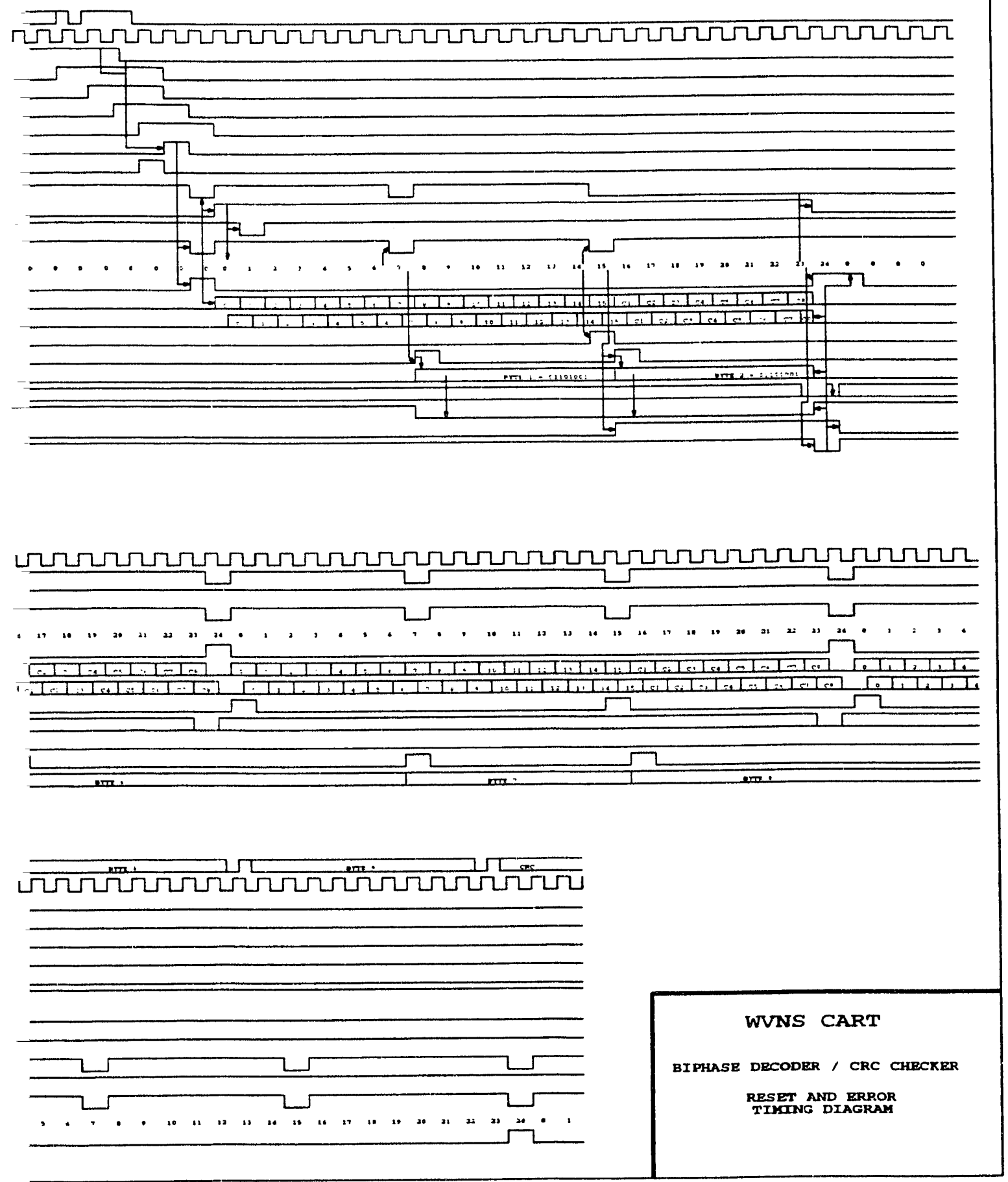

$-r$ reset and error timing diagram. 


\subsubsection{Serial-to-parallel converter}

U12 and U13 [U24 and U17] make up the serial-to-parallel converter section, which converts the serial data stream from the parallel-to-serial converter section back to its parallel form. In terms of the flow of data trrough the decoder circuits, the conversion to serial and back to parallel is not expressly needed; however, the CRC checker does need serial data, and the delay induced by the parallel-to-serial and serial-to-parallel conversions actually simplifies the timing requirements of the decoder. The delay is needed because the CRC check is completed only after the entire 24-bit data/CRC combination has been run through U21 [U30], and in order to align the error flag with the data before it is entered into the output storage section, the parallel data must be delayed by a minimum of 24-bit cells, which would require one extra register and its associated control lines and timing. U13 [U24] is a serial-input, parallel-output, shift register which is clocked by the inverted phase of the system clock. U12 [U17] is clocked by the control section and delays the data from U21 [U30] by 8 bits so that by the time the second of the two data bytes must be written to the output storage section, the error flag will have been received and will be ready to be entered along with the data. The CRC byte is not clocked into U12 [U17], although it is converted to parallel form by U13 [U24].

\subsubsection{Output storage section}

The output storage section stores the data bytes as they are clocked into U12 [U17]. U3 [U7] isolates the output of the FIFOs from the tri-state bus. When data are first clocked into the FIFOs their OR outputs go high, causing the output of U6C [U23C] to rise and signalling that a packet has been received. A negative pulse on /EOP will reset U26A [U31A], signifying that the packet has been decoded and checked for errors. Once /EOP is received, the data can be read out of the FIFO by pulling /RD low [high], which enables the outputs of U3 [U7], asserting the first data byte onto the bus. /RD must remain low [high] during the read operation. After the read operation is completed for each byte, /RD must be retumed high [low] because its rising [falling] edge after being inverted by U14E causes the next data byte in the FIFO to be clocked out. OR1 will fall during the period in which /RD is asserted and will retum high only if another data byte is ready to be read out of the FIFO. If OR1 does not return to the high state, the byte just read was the last one in the packet. The ERRFLG output from the FIFO is valid only while the even-numbered data bytes are at the FIFO output and should not be read with the odd-numbered bytes.

\subsubsection{Remote reset (cart only)}

The remote reset pulse is generated by the control section from the output of the remote reset comparator U18, which is an 8-bit TTL comparator. The reset code is set via the 16-pin header (arranged in an 8-by 2-pin grid) that feeds the Q-inputs of U18. For reliability purposes the pins of the header should be wire-wrapped in lieu of using jumpers. The output of the comparator is used by U26, which looks at the signal at a specific point in the decoding process to determine whether a reset pulse should be generated. If the first byte in a two-byte encoding pair matches the reset code, the RFLG output of U26 latches high. If the second byte in the pair also matches the code and the CRC checker does not detect an error in either byte, U26 will generate a single active-low reset pulse (INIT) that is 1 clock cycle $(8 \mu \mathrm{s})$ in length. /NIT is then driven by U29F to turn on Q1, which pulls down the PBRESET line on the computer's bus. /NIT also feeds back to reset the decoder circuitry via U23D. If the second data byte does not match the reset code or if the CRC checker detects an error, U26 will reset RFLG and write the two data bytes to the output storage section. 


\subsection{FACILITY COMRIUUNICATIONS SYSTEM CONTROLLER}

A single-board computer is designed to (1) handle communications encoding and decoding 'asks, (2) coordinate high-speed data passage, (3) switch on and off the facility transmit/receive units, (4) interface to the engineer's console computer, and (5) provide data buffers for serial data transmissions. The computer will use a STD bus and be mounted in a 19-in. card cage in the engineer's console, as shown in Dwgs. Q-6340-102 and Q-6340-103.

The control processing unit (CPU) is a Prolog Model 100,12-MHz computer with $512 \mathrm{kB}$ of memory ais a breakout board with two RS-232 ports. The CPU uses a disk operating system (DOS) so that software development time is minimized. There is a battery-backed RAM disk card, Prolog 7715A-03, that will hold $256 \mathrm{kB}$. A Prolog 7514 card interfaces the communications controller computer to the Allen-Bradley's remote I/O network. In this manner, PLC I/O points are emulated in the Prolog computer. The facility encoder/decoder card, described in Sect. 6.7, mounts in one slot of the communications controller STD rack. It interfaces to the Prolog computer through a Prolog 7508 digital interface card. The same 7508 card also interfaces to Opto-22 digital $\mathrm{I} / \mathrm{O}$ modules that are used to switch the facility antennas on and off, and to receive antenna usage status from the if system. The Opto-22 modules are mounted on another 19-in. panel in the back of the engineer's console.

\subsection{FACILITY ANTENNAS}

The facility antennas, shown in Fig. 6.10, are dipole antennas which are designed to fit within existing cell penetrations. The antennas fold to fit inside a 1 1/4-in.-ID straight-through penetration. Four of the facility antennas will be used. The facility antenna assembly is shown in Dwg. X3E020097A113. The main components of the antenna assembly are the base, antenna, insulator, spring, shaft, pin, collar and corrugated hose.

The base is machined out of PEEK (polyetheretherketone) thermoplastic, which also has good radiation tolerance. The base needs to be an insulator in order to not affect the transmissive properties of the dipole antenna. The shaft, made of 304 stainless steel, is pressed into the end of the base for mounting the antenna.

Each dipole antenna has two 1/8-in.-diam by 4-in.-long antenna rods of 304 stainless steel. Small copper tabs are silver soldered onto the end of the antenna rods. The coaxial cable leads are soldered to each of the copper tabs. These are then epoxied into the insulator. The insulator is also made of PEEK. The insulator fits over the shaft on the base and pivots to fold and deploy the antenna. A torsion spring is used to provide the force to deploy the antenna. A pin is also pressed into the base to provide a positive stop for the correct position of the deployed antenna.

The corrugated hose is made of 316 stainless steel. It is used to push the antenna through the cell penetration to deploy it in-cell and is also used as a conduit for the coaxial cable. The stainless steel collar is used to attach the corrugated hose to the base. Once inserted into the cell, the dipole antenna unfolds and deploys to the vertical orientation for signal transmission with the cart. 

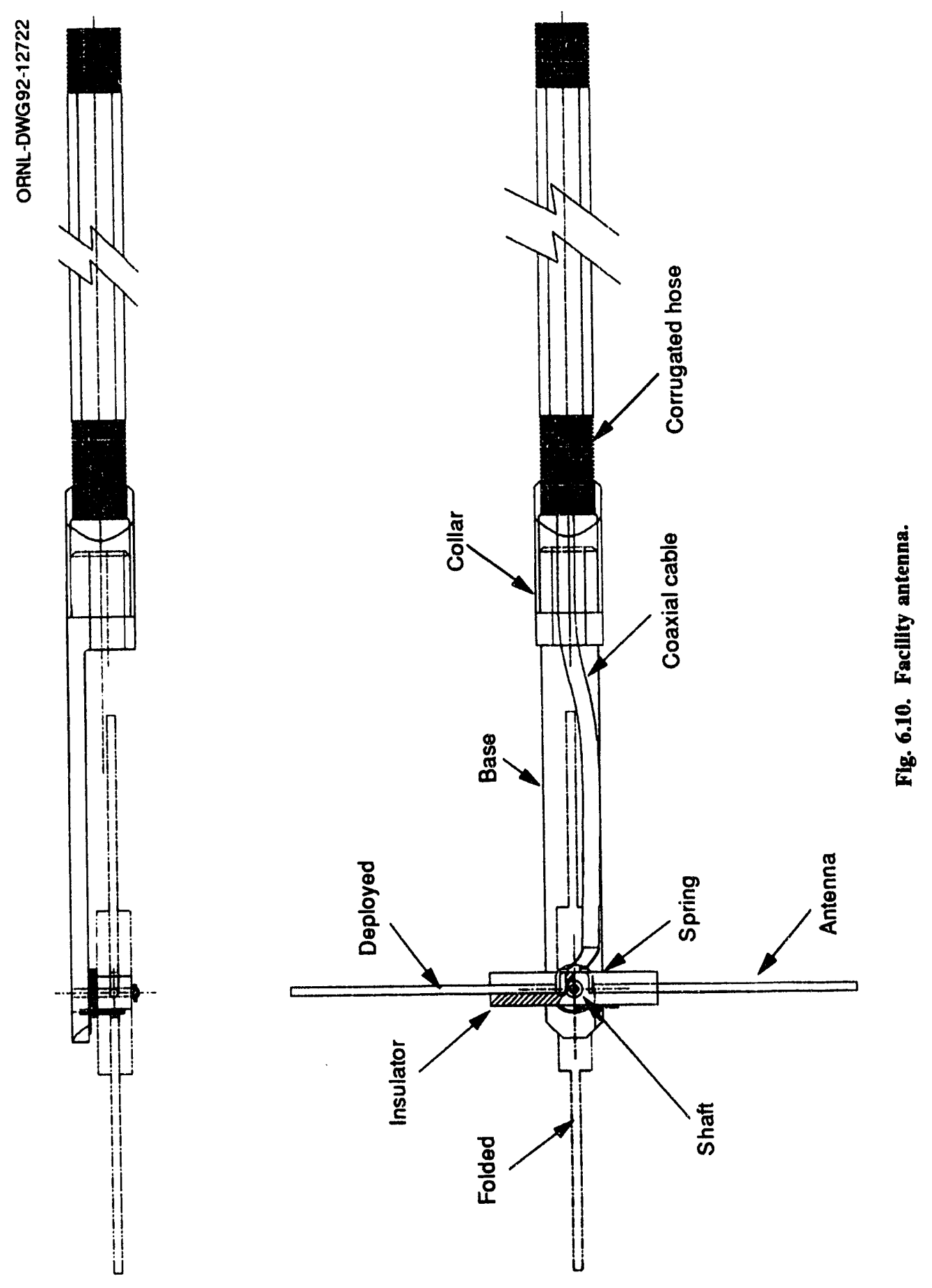


\section{CART ELECTRONICS SYSTEM}

This section describes all cart-mounted electronics systems except for the communications hardware. Figure 7.1 shows the basic layout of printed circuit cards in the cart electronics system. Note that only the WinSystems and interface module boards are discussed in this section. The remaining boards (communications system boards) are covered in Sect. 6. A wiring diagram of the cart electronics is given in Dwg. Q-6340-215.

\subsection{SYSTEM CONTROLLER}

The system controller is based on the STD bus using an embedded controller manufactured by WinSystems Inc. The embedded controller card, a WinSystems single-board computer (Model LPM-SBC40R-8-BAT), uses an Intel 8086 microprocessor with the WinSystems read-only memory (ROM) disk operating system. In order to provide additional digital I/O lines, the singleboard computer is fitted with a piggyback I/O expansion board (WinSystems model number SBXPIO). Section 9.4 provides a detailed description of the cart control software and communication protocols.

The system controller is designed to withstand a $10^{7}$ rad total integrated dose using a lead shielding enclosure. See Appendix B for information on the radiation test of the system controller. A diagram showing the system hardware configuration is given in Dwg. Q-5340-247.

\subsubsection{Interface-to-Cart Communications System}

The system controller decodes, processes, and responds to commands from the cart communications system and passes data from the cart electronics system through the cart communications system to the facility system. The system controller is tied to the communications system via the cart biphase encoder/decoder. This connection is made through the SBX-PIO digital I/O card and provides the system controller with decoded data from the facility system and allows the system controller to send encoded data to the facility system. Connections are also made from the system controller through the SBX-PIO card to the communications system transmit/receive switching circuitry. Timing of receive/transmit cycles are software controlled (see Sect. 9.4 for a discussion of the controller software routines).

\subsubsection{Interface to Motor Drives}

The system controller provides control signals, direction control, and pulse-width modulation signals to the motor drives via the cart controller interface board. The pulse width modulation signal allows software control of cart speed. If the system controller receives a motor overcurrent trip signal from the controller interface, an error message will be sent to the facility system (via the communications system) to notify the cart operator of the problem. The operator has the option of sending a command that will re-enable the tripped motor drive. If the abnormal 


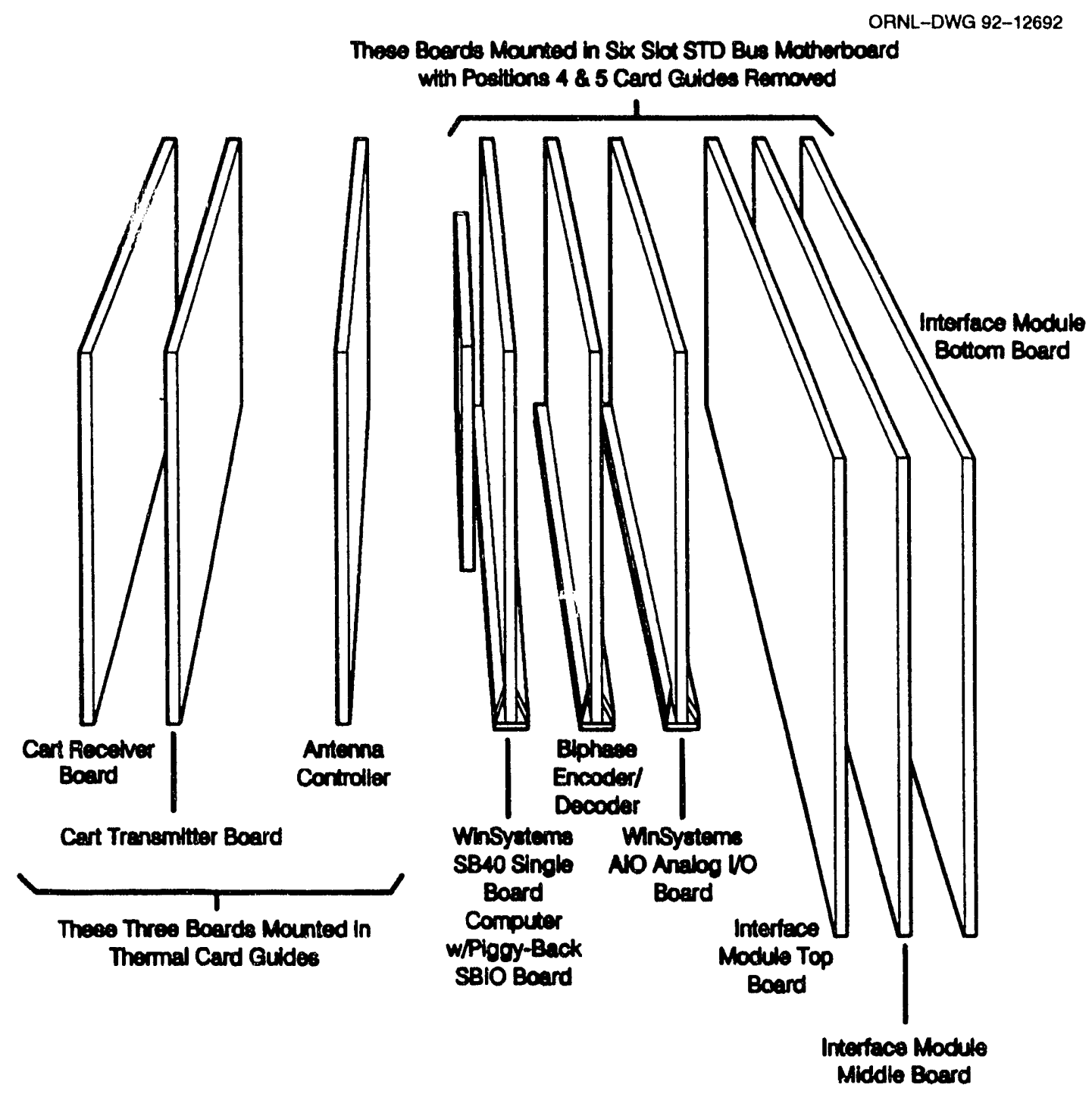

Fig. 7.1. Cart electronics rack card arrangement.

condition persists, the particular motor drive will again be disabled and the operator notified. Note that while motors are controlled by the system controller, the motor overcurrent trip and motor disabling circuits are located on the controller interface board (see Sect. 7.3) and are being directly activated by an overcurrent condition.

\subsubsection{Interface to Analog-to-Digital Converter}

Data from the analog-to-digital (A/D) converter must be controlled, received, and stored for transmission by the system controller. The A/D converter is located in the STD-bus section of the cart electronics, and communication with the A/D is accomplished through the STD interface. See Sect. 9.5 for a description of signals processed by the $A / D$ converter. 


\subsubsection{Interface to Cart Interface Module}

Much of the signal processing and high-current switching for the cart is carried out by the cart interface module, which is described in Sect. 7.3. The system controller is tied to the interface module through 14 lines of digital output and 7 lines of digital input. The system controller also has access to 17 analog signals via the A/D converter board from the controller interface.

Digital signals to the interface are the remote outlet controls, fan control, motor direction control, motor resets, and pulse-width modulation signals for each motor. Digital signals from the interface are the door limit switch detected, outlet trip signals, motor trip signals, and the interface module ID.

Analog signals from the interface are the controller temperature, battery compartment temperature, outlet voltage signals, outlet current signals, motor voltage signals, motor current signals, battery voltage signal, and battery current signal.

\subsection{MOTOR DRIVES}

The motor drive circuit is a standard H-bridge type MOSFET drive. Drive signals for the solid state switches are provided by Maxim driver integrated circuits located on the controller interface board (see Sect. 7.3). Signals for the motor drives originate in the system controller board and are buffered by the controller interface board. A separate motor drive circuit board is provided for each motor.

The motor drive circuit schematic, parts placement and layout, is given in Dwg. Q-6340-235. The parts list for the motor drive is given is Dwg. Q-6340-236.

\subsection{CART INTERFACE MODULE}

The cart interface module controls battery power distribution during charging and provides buffering for all current shunts and voltage signals, buffering for the temperature sensors located in the battery and controller compartments, motor and outlet overcurrent trips and resets, buffering for the threshold detector switch, a controller identification (ID) output, current shunt span calibration signals, a drive signal to the fans, and motor drive signals. A functional block diagram for the controller interface is shown in Fig. 7.2. Section 7.1.4 has a detailed list of signal lines from the controller interface module to the system controller. A schematic drawing of the overall wiring for the interface module is given in Dwg. Q-6340-215.

The cart interface module is composed of three separate circuit boards which are colocated in the cart electronics rack assembly. Since the boards are configured in a stacked arrangement, they are referred to as the top, middle, and bottom interface boards in documentation. The schematic diagram, printed circuit board layout, and parts list for the interface module top board are given in Dwgs. Q-6340-220 through Q-6340-224. The schematic diagram, printed circuit board layout, and parts list for the interface module middle board are given in Dwgs. Q-6340-225 through Q-6340-229. The schematic diagram, printed circuit board layout and parts list for the interface module bottom board are given in Dwgs. Q-6340-230 through Q-6340-233.

\subsubsection{Battery Switching During Charging}

The cart system controller and other cart electronic systems are de-energized by the interface module during battery charging. Charging is initiated when a voltage greater than the battery voltage is sensed on the charging shoes. Note that the voltage must be of the proper polarity for 


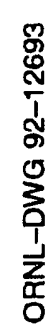

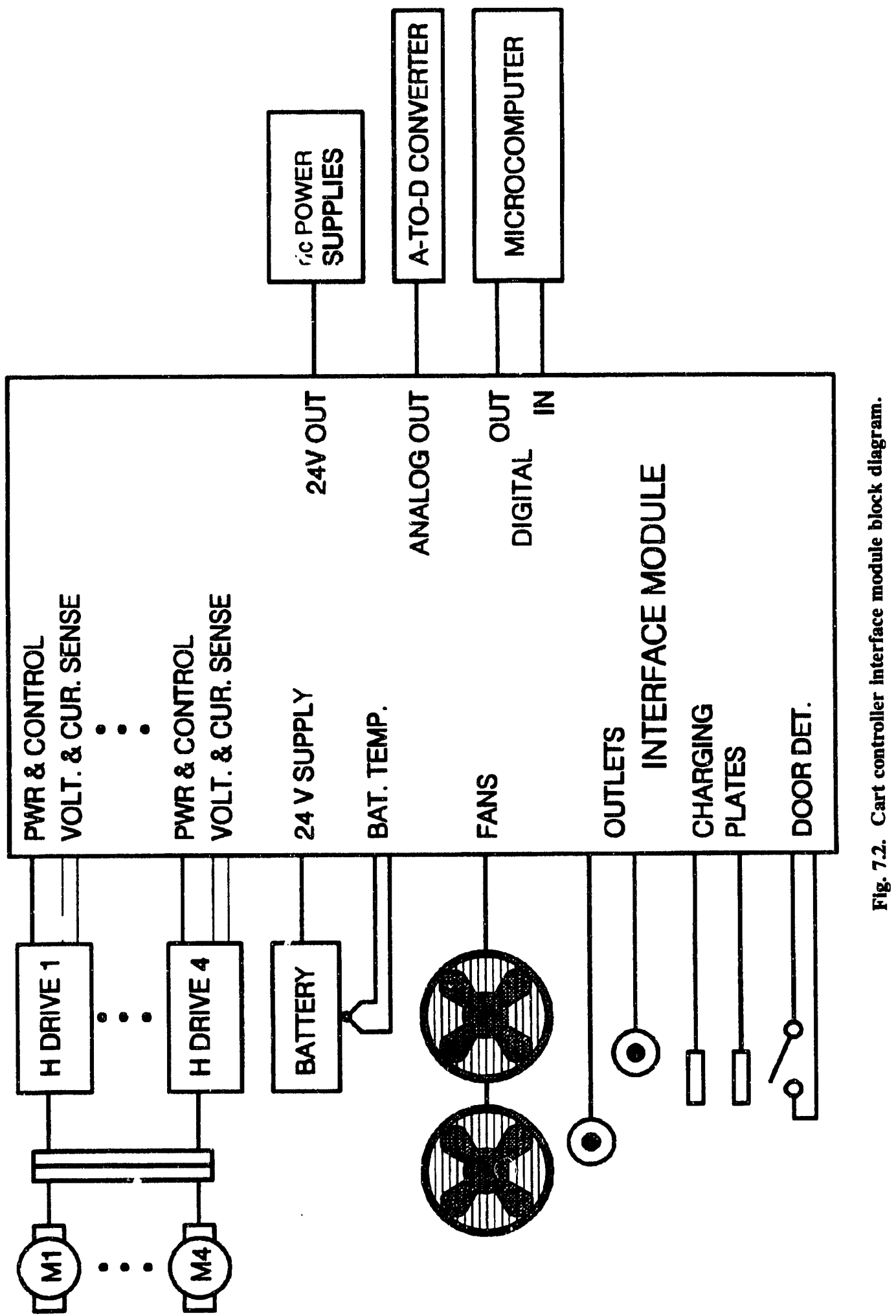


the controller interface to allow charging. Once charging is initiated, it is terminated only when the current from the charger is less than a programmed level $(\sim 80 \mathrm{~mA})$. This is accomplished by a high-gain current monitor located in the battery and charger interface circuit (see Dwg. Q-6340-222) which allows detection of the battery charging current. Switching is accomplished as shown in Fig. 7.3.

\subsubsection{Current Shunt and Voltage Buffers}

All current shunt and voltage signals passed to the A/D converter card are buffered in the interface module. The buffers are built using the Precision Monolithics AMP-03 unity gain differential amplifier. Input protection is provided by a resistor/voltage clamp circuit at the input of each buffer. Buffer schematics are given in Dwg. Q-6340-232.

\subsubsection{Temperature Monitors}

Two temperatures are monitored in the cart electronics enclosure. These are the battery compartment temperature and the cart controller temperature. Figure 7.4 shows a block diagram of the temperature measurement system. The battery compartment temperature is measured with a Type $\mathrm{E}$ thermocouple washer ring which is mounted under the negative battery electrical terminal contacts. The cold junction compensation for the thermocouple and controller temperature are both measured by the same solid state temperature sensor located on the interface module board. As indicated in the figure, the controller temperature is used to control fan operation. A schematic for the temperature mositoring circuit is given in Dwg. Q-6340-227.

\subsubsection{Overcurrent Trips}

The output signal from each motor and outlet current shunt is sensed after buffering. A threshold detection circuit is used to produce a digital signal that causes any motor or outlet to be disabled if the current exceeds a resistor programmed level. The trip condition is latched, causing the motor or outlet to remain disabled until reset by the system controller. Resets from the sy: 'em controller are received via the communication system from the engineer's console. A schematic for the fault detection circuit is given in Dwg. Q-6340-227.

\subsubsection{Threshold Detector Switch}

The threshold detection switch actuation is sensed via a pull-up resistor located on the interface module board. The signal from the pull-up resistor is passively buffered prior to being passed to the system controller from the interface board. The detector switch buffer schematic is given in Dwg. Q-6340-227.

\subsubsection{Controller ID}

A 2-bit (0 to 3) controller ID number is user programmed by a two-section dip switch (S1) located on the top interface module board. This allows each electronics enclosure to be identified and addressed independently by the facility system. 


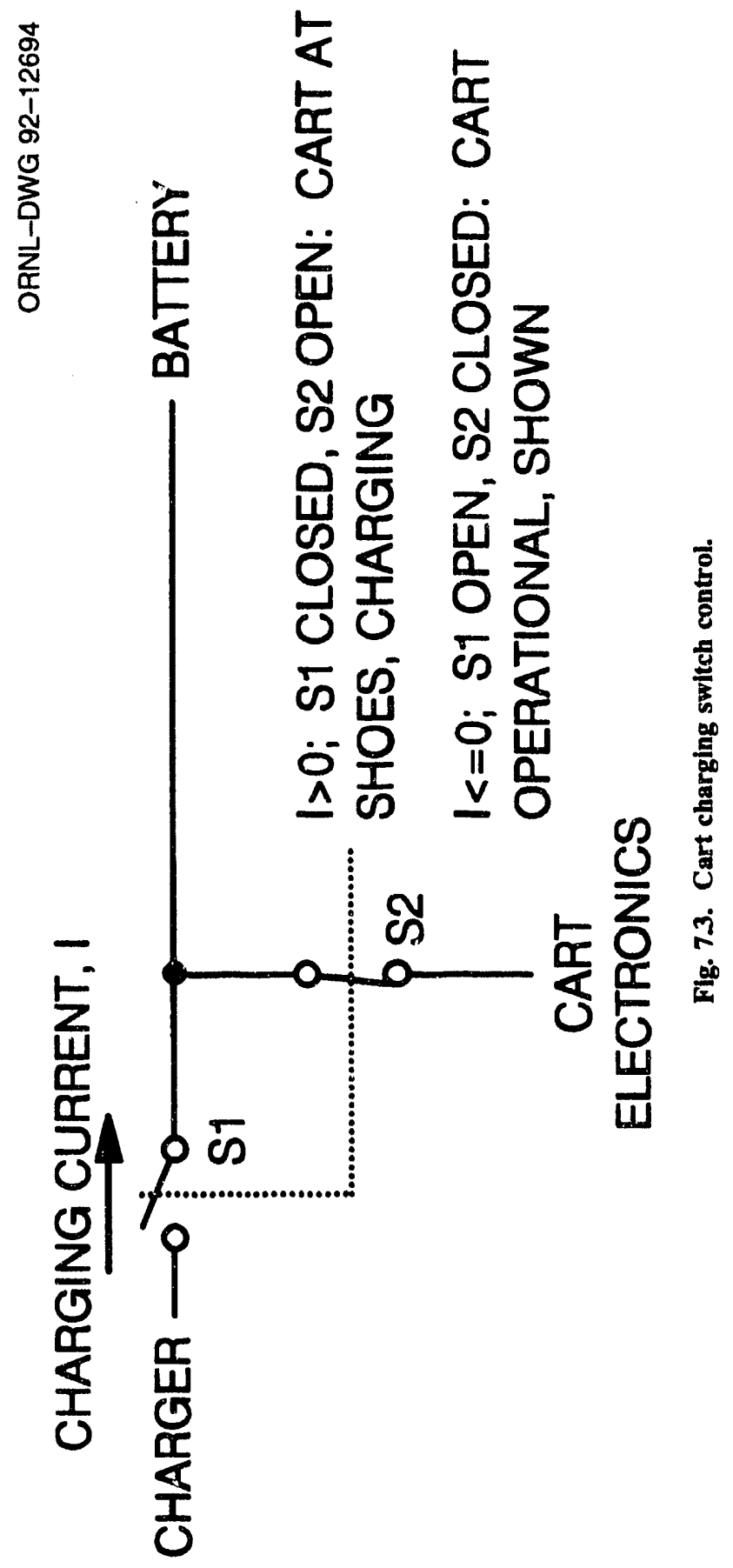




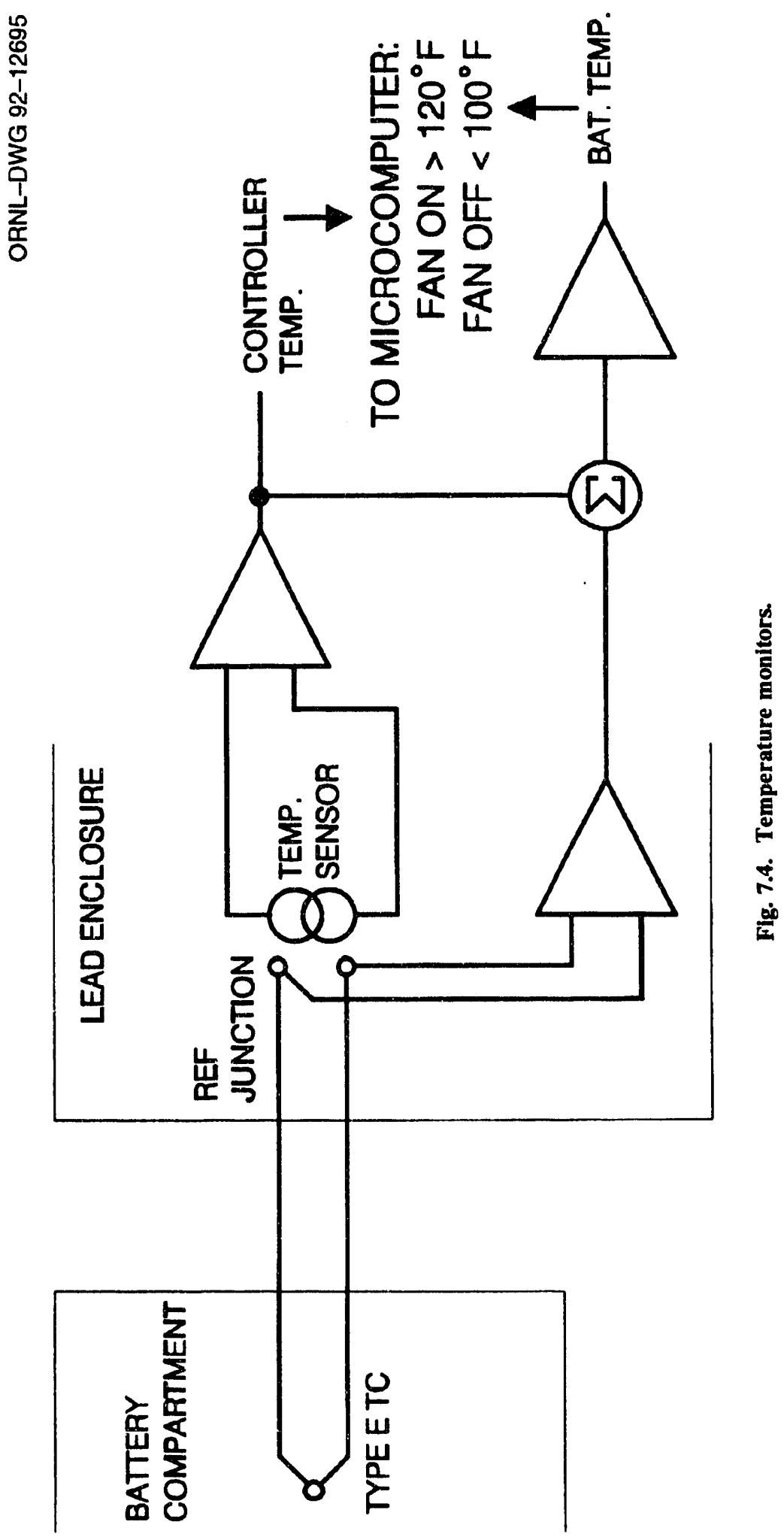




\subsubsection{Current Shunt Span Calibration}

Figure 7.5 shows a block diagram of the current shunt span calibration circuit. The 5-A precision current source is successively applied to each motor and outlet current shunt. The system controller uses the known 5-A signal to compute the span coefficient for each current channel used in all subsequent current logging. An out-of-range reading will cause the system controller to flag a current channel as bad. Calibration occurs at system bootup and after a system reset is issued by the engineer's console via the communications system. A schematic for the shunt span calibrator is given in Dwg. Q-6340-232.

\subsubsection{Fan Control}

The fan is controlled by the system controller through the interface module. A software routine in the system controller monitors the controller enclosure temperature from the interface module turning the fan on at $120^{\circ} \mathrm{F}$. The fan is cycled off at $100^{\circ} \mathrm{F}$. A buffer on the interface module provides drive to the fan. See Dwg. Q-6340-227 for a diagram of the fan interface circuit.

\subsubsection{Motor Drives}

For each motor drive there are independent control and overcurrent trip signals. Independent signals are used for each motor drive to prevent one failed motor drive from affecting the other motor drives. The motor drive is a standard H-bridge-type drive. Pulse-width modulation signals from the system controller are used to drive the H-bridge through the controller interface based on the motor direction command signals. Motor drive schematics are given is Dwg. Q-6340-223.

\subsection{A/D CONVERTER SYSTEM}

The A/D converter is a WinSystems Model LPM-AIO-DC. All data transfers to and from the converter occur over the STD bus. See Sect. 9.5 for a description of signals processed by the A/D converter.

\subsection{DOOR THRESHOLD LIMIT SWITCH}

The door threshold limit switch is used to detect door threshold crossing during cart motion. The threshold limit switch provides a contact closure when activated by a door threshold. The contact closure is buffered by the cart interface module to protect the system controller. Details for the threshold limit mounting are given in Dwg. X3E-020097-A102.

\subsection{BATTERY}

The final cart design will use two Powersonic Model PS-12400, 40 A-h 12-V batteries wired in series. Based on data obtained during testing of these batteries (see Appendix C), they are capable of providing roughly $2.92 \mathrm{MJ}$ for a 1.75 -A cart idle current at $20^{\circ} \mathrm{F}$. From measurements made during prototype testing, we estimate that the cart will consume roughly 2 A during idle. Derating (linearly) for 2-A operation and assuming that only $80 \%$ of battery energy will be available at the end of battery life gives an available battery energy of $2.05 \mathrm{MJ}$. For a 2-A idle current, cart energy consumption is summarized in Table 7.1. Therefore, the Powersonic batteries should provide for more than 2 typical cart cycles ( 4.5 -h duration per cycle) or an operating time margin of $5 \mathrm{~h}$. 


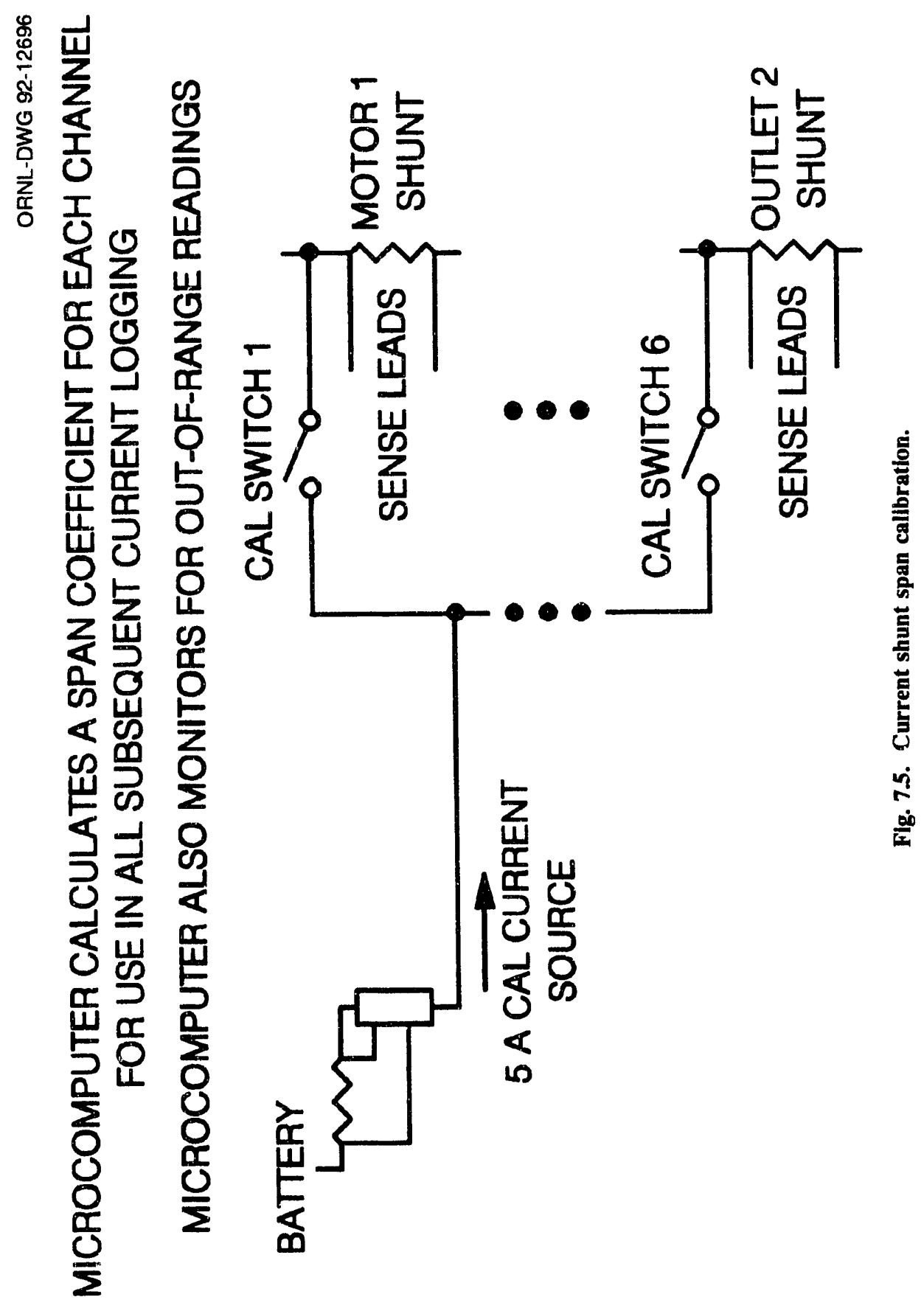


Table 7.1. Summary of cart energy requirements for 1 cycle based on prototype testing

\begin{tabular}{lccccc}
\hline \multicolumn{1}{c}{ Operation } & $\begin{array}{c}\text { Time } \\
(\mathrm{min})\end{array}$ & $\begin{array}{c}\text { Drive } \\
\text { power } \\
(\mathrm{W})\end{array}$ & $\begin{array}{c}\text { Electrical } \\
\text { power } \\
(\mathrm{W})\end{array}$ & $\begin{array}{c}\text { Total } \\
\text { power } \\
(\mathrm{W})\end{array}$ & $\begin{array}{c}\text { Energy } \\
(\mathrm{kJ})\end{array}$ \\
\hline Idle time & 255 & 0 & 50 & 50 & 765 \\
Moving, no load & 6.96 & 92.9 & 50 & 142.9 & 59.7 \\
Moving, full load & 7.47 & 251 & 50 & 301 & 135 \\
$\quad$ Totals & 269.4 & & & & 960 \\
\hline
\end{tabular}




\section{CONTROL MODULE ENCLOSURE ASSEMBLY}

The control module enclosure assembly contains all of the on-cart controls and electrical equipment to perform all of the cart control functions within a single assembly. The control module enclosure assembly is designed as a remotely replaceable module. The assembly is held in place on the cart by two captive bolts which can be operated by a remote impact wrench. The lifting bail is provided for remote handling by the crane for removal and installation. Alignment guides will be provided on the cart design by WVNS for gross positioning of the assembly. These should provide alignment of the assembly for coupling of the remote electrical connector. A dowel pin in the remote electrical connector provides the final alignment of the assembly. All of the electrical connections between the control module enclosure assembly and the transfer cart are contained in this connector. In the event of failure of any of the cart controls, the entire module can be removed and replaced remotely using only the crane and impact wrench.

Two views of the control module enclosure assembly are shown in Fig. 8.1 and in Martin Marietta Energy Systems Engineering Dwg. X3E020097A102, "WVNS Vitrification Facility Transfer Cart Control Module Enclosure Assembly." The subassemblies included in the control module enclosure assembly are listed as follows and are described in the following sections.

1. Enclosure module

2. Battery compartment assembly

3. Front cover and threshold limit switch

4. Bail assembly

5. Antenna assemblies

6. Shielded electronics enclosure

7. Remote electrical connector assembly

8. Battery charging plate and insulator

The drawings referred to in the following descriptions are Martin Marietia Energy Systems Engineering drawings and are contained in Appendix E.

\subsection{ENCLOSURE MODULE}

The enclosure module weldment is shown in Dwg. X3E020097A103. The enclosure is an all-welded structure made up of $1 / 2$ - and 3/8-in.-thick 304 stainless steel plate. The enclosure design provides two separately sealed compartments. The upper space is for the batteries, which are in a sealed compartment attached to the top cover. The lower space is for electronics which require shielding from the radiation. Both of these are described in more detail below.

The stainless steel enclosure is completely sealed to prevent moisture or nitric acid vapor from entering the electronics or battery compartments. Covers over the top and front compartments are sealed with $\mathrm{O}$-rings. $\mathrm{O}$-ring grooves are machined into the sealing surfaces of the covers. The top cover serves a double purpose as (1) an enclosure for the top and (2) the top of the battery 


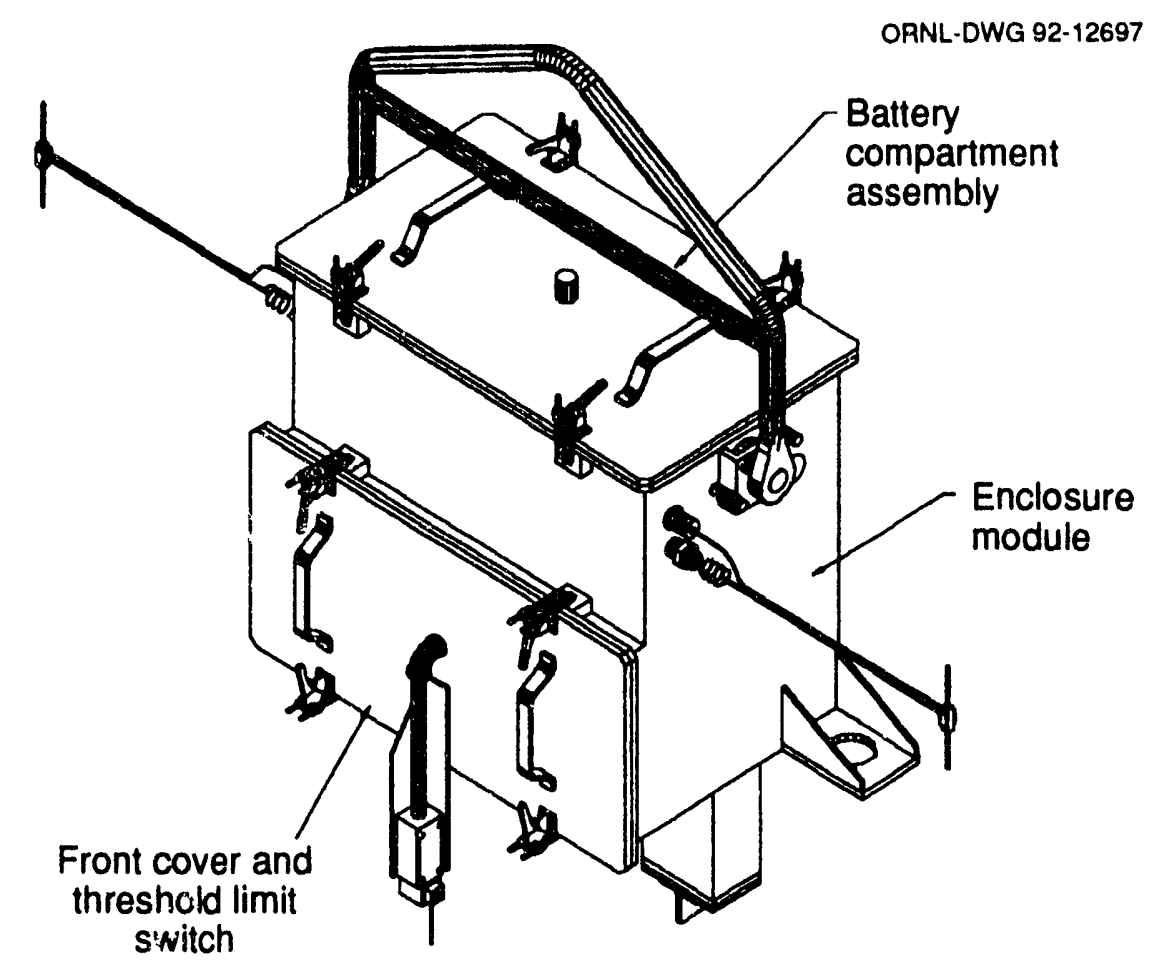

Note: Shielded electronics enclosure (not shown)

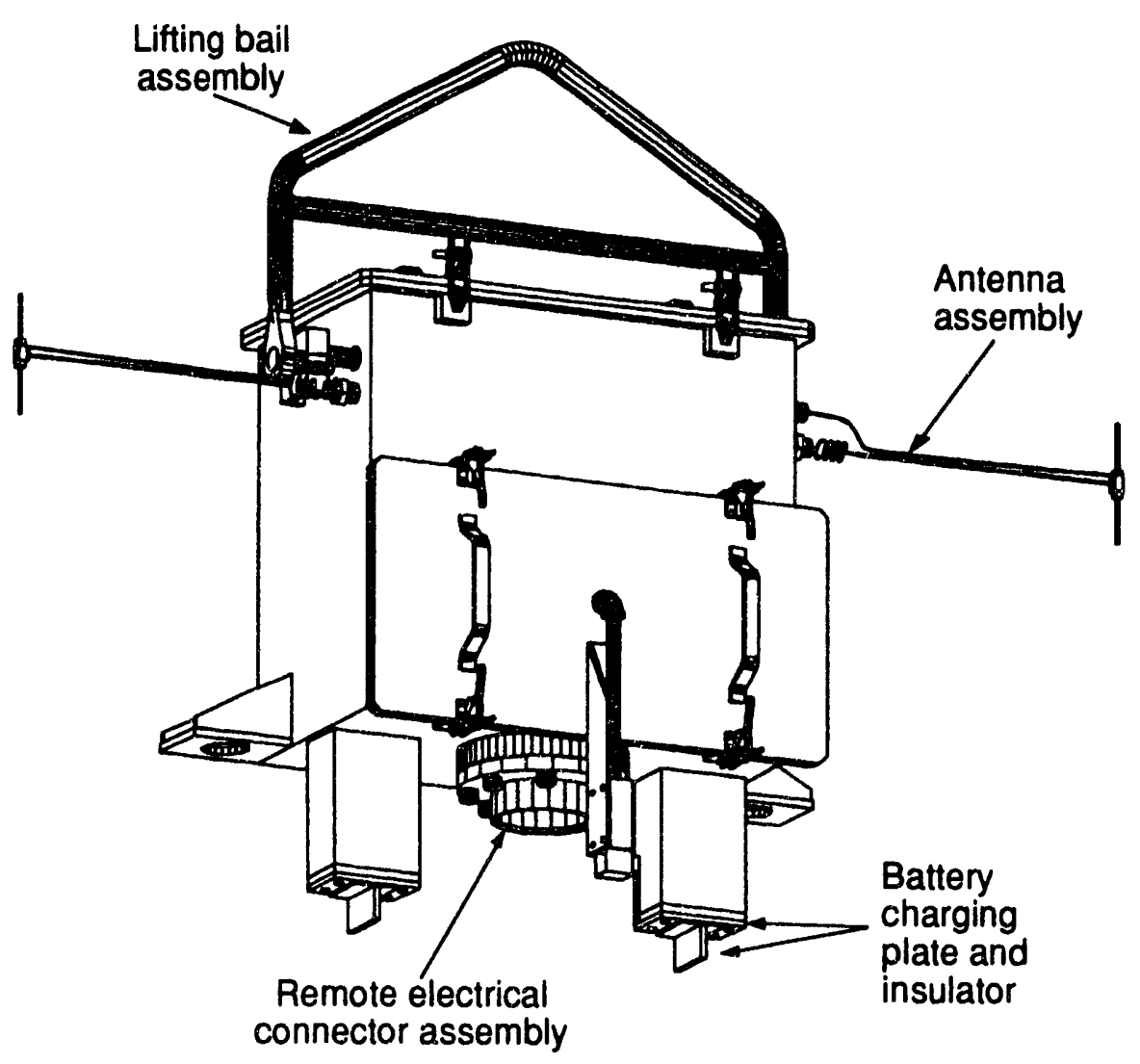

Fig. 8.1. Two views of the control module enclosure assembly. 
compartment. The front cover can be removed to provide access to the wiring hamess and the motor driver circuit boards. Each cover is held in place by locating pins and four toggle action clamps for easier maintenance. All components on this assembly and within the enclosure are designed to be contact maintained.

On the bottom of the enclosure, two legs, fabricated from rectangular stainless steel tube, extend down for mounting the battery charging plates. The mounting location for the remote electrical connector is located between the legs on the bottom of the enclosure. Access holes are provided in the bottom of the enclosure for cabling to each leg and to the electrical connector. The electrical connector and charging plates are bolted to the enclosure and are sealed with O-rings.

A false bottom and partition are provided inside the enclosure to locate and support the shielding enclosure. The false bottom provides space for routing the cable hamess under the shield enclosure to the remote electrical connector and to each of the charging plates.

On each side of the enclosure near the top are two electrical connectors and a stainless steel boss for mounting the antenna. The electrical connectors are Lemo-type bulkhead connectors, hermetically sealed to the enclosure, with all wiring inside the enclosure. The connector nearest the antenna boss is for the antenna cable. The other connector is for the auxiliary electrical outlet. The antenna mounting boss is welded to the side of the enclosure and has internal threads for the antenna to screw into.

On each side near the bottom of the enclosure are the mounting locations for the captured remote hold-down bolts.

\subsection{BATTERY COMPARTMENT ASSEMBLY}

The battery compartment assembly is a sealed stainless steel enclosure composed of the Enclosure Module top cover and a battery box. The battery compartment assembly is shown in Dwg. X3E020097A105. The battery box is fabricated of 7-gage 304 stainless steel sheet. The battery box is fastened to the top cover using $241 / 4$-in.-diam by $3 / 4$-in.-long studs welded to the underside of the top cover.

A small high-efficiency particulate air filter is provided in the top cover of the assembly to vent hydrogen which may be generated during recharging of the batteries. Two handles are also provided on the top cover to facilitate handling for removal or replacement of the battery module. As mentioned previously, the battery compartment assembly is fastened to the enclosure module by four toggle action clamps on the cover. Two locating pins are used to position the top cover on the enclosure.

Inside, the compartment will contain two 12-V, 40-A-h sealed lead-acid rechargeable batteries. An Amphenol/Bendix bulkhead connector is provided in the end of the battery box for connection to the controls wiring hamess while keeping the battery enclosure sealed. Also inside the compartment at one end is a lead shield block encased within 7-gage stainless steel. This block is located directly above the ventilation hole in the shield enclosure to help shield the electronics within the shield enclosure.

\subsection{FRONT COVER AND THRESHOLD LIMIT SWITCH}

The front cover provides access to the wiring harness and the motor driver circuit boards. The front cover also supports the threshold limit switch. The front cover and threshold limit switch are shown in Dwg. X3E020097A106. The front cover is 3/8-in.-thick stainless steel plate. The sealing surface of the plate is machined with a groove for an $\mathrm{O}$-ring seal. The extemal surface of the cover has two handles for removal or replacement. As mentioned previously, the front cover is fastened to the enclosure module by four toggle action clamps. Two locating pins are used to position the cover on the enclosure. 
The threshold limit switch is used to detect door threshold locations to correct errors in the cart position algorithm. The threshold limit switch is mounted on a stainless steel angle bracket welded to the front cover. This bracket positions the limit switch trip lever at the elevation of the door thresholds. A $1 / 2$-in.-diam stainless steel pipe coupling penetrates the front cover for the limit switch cable. The pipe coupling is welded to the front cover, and the cable is sealed in conduit from the coupling to the limit switch. A connector is provided in the limit switch cable inside the front cover to ease removal of the cover.

\subsection{BAIL ASSEMBLY}

The lifting bail assembly is provided at the top of the enclosure to allow the facility crane to lift the entire assembly for remote removal or replacement. The bail assembly is shown in Dwg. X3E020097A106. The bail assembly consists of the bail, bail mounting bracket, and hinge pin.

All of the bail assembly parts are fabricated from 304 stainless steel. The bail is a weldment of 1 1/2-in. pipe, 1-in. pipe, and a rod end. The bail mounting bracket has a hole for the hinge pin and stops to hold the bail somewhat vertically for pickup by a crane hook. The hinge pin is inserted through a hole in the mounting bracket and is welded into the bail. The mounting bracket will be located at the center of gravity of the Control Module Enclosure Assembly and welded.

\subsection{ANTENNA ASSEMBLIES}

Two dipole antenna assemblies are provided, one on either side of the enclosure. The cart antenna assembly is shown in Dwg. X3E020097A111. The antennas extend past the edge of the transfer cart for line-of-sight communications with the facility antennas. The antennas have a vertical orientation. See Sect. 3 for details on the cart communication system. The antenna assembly consists of the spring arm weldment, lock nut, antenna, insulator, antenna bracket and coaxial cable.

The spring arm weldment consists of a 3/16-in.-diam spring arm welded into a threaded fitting. The antenna bracket is 16-gage stainless steel and is welded on the other end of the spring arm. The lock nut assembles onto the threaded fitting, which screws into the boss on the enclosure module. All of these components are 304 stainless steel.

Each dipole antenna has two 1/8-in.-diam by 4-in.-long antenna rods of 304 stainless steel. Small copper tabs are silver soldered onto the end of the antenna rods. The coaxial cable leads are soldered to each of the copper tabs. These are then epoxied into the insulator. The insulator is made of PEEK thermoplastic, which also has good radiation tolerance. The insulator is pinned to the antenna bracket with a stainless steel spring pin.

\subsection{SHIELDED ELECTRONICS ENCLOSURE}

Electrical components not capable of withstanding $10^{7}$ rad are protected in a shielded enclosure with the equivalent shielding of 3 orders of magnitude of reduction. The shielded enclosure is made up of lead encased in a stainless steel liner. The shielded enclosure weldment is shown in Dwg. X3E020097A112. The shielded enclosure weldment consists of three parts: the side shield, the top shield, and the base shield.

All three parts of the shielded enclosure weldment are of similar design. They provide a shield wall thickness of 2.62 in. of lead, lined with 11-gage 304 stainless steel sheet. The base shield, the main shield enclosure, provides a volume of approximately $18 \mathrm{in.}$ long $\times 11$ in. wide $\times$ 10.5-in. deep for installing the cart control electronics. The top of the base shield enclosure is

open to insert the electronics rack. Another opening is provided on the side of the base shield for 
routing of the wiring harness. The side shield fits adjacent to the base shield and provides shielding over the side opening and space for the wiring harness. The top shield fits in the top of the base shield and rests on a ledge inside the side walls of the base shield. It has two handles to remove the lid for access to the electronics rack. The top shield also has a small opening for ventilation only. This opening is located above a cooling fan in the electronics rack to circulate air through the electronics and within the enclosure module. The top of the electronics rack is designed to rest on the ledge of the side walls and is held in place by the weight of the top shield.

\subsection{REMOTE ELECTRICAL CONNECTOR ASSEMBLY}

The control module enclosure assembly interfaces with the transfer cart via the remote electrical connector assembly on the bottom surface. The remote electrical connector is a Hanford Purex-type connector and is shown in Dwg. X3E020097A107. The remote electrical connector assembly consists of the flange, insulator, plug pins, and pin connectors.

The flange is machined from 304 stainless steel. The insulator is a Lexan plate which bolts to the flange. It provides an array of nine plug pins; two pins for each of the four drive motors ori the cart plus one for cart ground. The pin connectors are threaded onto the back side of the plug pins. The wiring is soldered into the pin connectors. The pin connectors are sized to handle approximately $90 \mathrm{~A}$, which exceeds the motor stall current. The pins and the insulator are both sealed within the flange using room temperature vulcanization seal compound. The flange bolts to the enclosure and seals with an O-ring.

\subsection{BATTERY CHARGING PLATE AND INSULATOR}

The battery charging plate and insulator are mounted on the bottom legs of the enclosure. They are positioned to contact the floor mounted charging shoes located between the rails. The battery charging plate and insulator are shown in Dwg. X3E020097A109.

The battery charging plate is a weldment of two 3/8-in.-thick 304 stainless steel plates forming a "T" shape. A $1 / 4$-in.-diam stud is welded to the top plate for the electrical terminal. The insulator plate is $3 / 4$-in.-thick ABS plastic. The charging plate bolts to the insulator plate, with the terminal extending through the insulator. The insulator bolts to the bottom of the enclosure legs and seals with an O-ring seal. The wiring and electrical connections are sealed inside the enclosure legs. 


\section{SOFTWARE DESCRIPTION}

This section describes the system software design for the transfer cart control system. Software will be part of the design of four components of the control system: (1) the engineer's console computer, (2) the PLC, (3) the facility communications controller, and (4) the cart controller. Sections 9.1-9.4 describe software in each of these systems. Drawings that should be referenced in these sections include Dwgs. Q-6340-110,111, 112, 160, and 210. Section 9.5 describes the communications protocol and command definitions used to communicate between the facility and cart systems.

The software described in this section is low-risk type. The control and data acquisition software is used to physically operate the cart and to generate operating and maintenance diagnostic data. Failure of the cart software can result in only one of two cart failure modes: (1) failure of the cart to operate or (2) unexpected cart operation. For the first mode, cart failure would only impact facility operations associated with waste canister transfer and result in delayed operations. For the second mode, failure of any software described here will not harm personnel, the environment, or equipment. The cells in which the cart travels are not usually occupied by personnel, with the exception of the EDR, where maintenance personnel may enter for maintenance of the cart. Slow speed of the cart and sweeps around the lower portion of the cart lower the probability that maintenance personnel would be injured by unexpected motion of the cart. Impacts on the environment are not foreseen in any mode of failure because the canisters which contain vitrified waste are held in place on the cart with guides and would not be disrupted in the case of a collision. Equipment that could possibly be impacted by the cart in the case of a runaway cart are cell doors and stops located at the ends of the tracks. Both the doors and the stops can withstand the full impact of the cart at its maximum design speed.

\subsection{ENGINEER'S CONSOLE COMPUTER INTERFACE SOFTWARE}

Refer to flow chart Dwg. Q-6340-110. Software will be running at the engineer's console, an industrially rugged IBM-compatible computer, to provide a man-machine interface between the engineer and the transfer cart control system. The interface software will be Intouch, a commercially available program that has been configured specifically for this application. The engineer will be able to request data trending, alarm and event summaries, and cart status displays from the computer. The computer will log historic data to disk at specific intervals including motor current and voltage; battery current, voltage, and temperature; auxiliary outlet voltage and current; and percent usage of system antennas. Furthermore, the engineer will be able to enter certain cart system commands into the computer that will be processed by the PLC. These commands consist of changing the ID of the cart electronics system with which to communicate, disabling individual facility antennas, requesting a reboot of the cart computer, and increasing or decreasing cart speed. The engineer's computer is not essential to the operation of the cart. Although it is important to $\log$ data for diagnostic and operational reference reasons, the cart can 
operate through use of the operator's pendants without the engineer's computer. Refer to Appendix $F$ for typical operations that can be performed at the engineer's console.

\subsection{FACILITY PLC SOFTWARE}

The Allen-Bradley PLC is the master of the cart control system. It directs the operation of all other system components either directly through its own hardwired inputs and outputs or through use of the facility communications controller. Software running on the PLC has been defined in a fundamental fashion in Dwgs. Q-6340-111 and Q-6340-112. Algorithms that require more explanation than provided in the drawings are described here. A ladder logic listing should be referenced for more detail.

\subsubsection{Cart Position Calculation}

The cart position calculation is based on which direction the cart is driving and the duration of the drive request from the operator's pendant. The position will be reported in feet from facility charging shoes to the cart's threshold limit switch. Positive position refers to distance north of the charging shoes, while negative position refers to distance south of the charging shoes, as shown in Fig. 9.1. In the figure, $A$ is the distance to door $63 \mathrm{M}-001, B$ is the distance to door $63 \mathrm{M}-008$, $\mathrm{H}$ is home position, and $\mathrm{C}$ is the distance to door $3 \mathrm{M}-3$ (a negative distance). A timer will be used to produce a pulse every $4 \mathrm{~s}$ when a valid drive command is in effect. To provide greater resolution, this may be changed to ten pulses every $4 \mathrm{~s}$. At a nominal cart speed of $15 \mathrm{ft} / \mathrm{min}$, the cart travels $1 \mathrm{ft}$ every $4 \mathrm{~s}$. Therefore, the timer pulse can be used by a counter to count the number of feet the cart has moved. By combining a count-up block with a count-down block, the calculated distance can be made to increase and decrease based on cart direction and cart drive commands.

\subsubsection{Corrections}

The cart position algorithm is based on the duration of the cart drive request and the nominal speed of the cart. It is necessary to have correction capability in the cart distance calculation for three reasons: (1) the cart speed will change based on cart load, (2) an acceleration period will occur before reaching nominal cart speed, and (3) the cart will coast after the drive command ceases.

Two correction modes will be used in the cart position algorithm. The first mode is based on resetting the position when the cart is at the charging shoes. The second mode is based on resetting the cart position to the distance of door thresholds from home position by noting when the cart's limit switch is actuated by the door thresholds.

- Home position. The home position will be confirmed by input from the charging shoes limit switch. When the cart-at-shoes input is received by the PLC, all timers and counters associated with the cart position algorithm will be reset. The position reference point on the cart is the door threshold limit switch. This switch is $-1 \mathrm{ft}$ from the charging plates; therefore, the home position reported will be either $\pm 1 \mathrm{ft}$, depending on which direction the cart is oriented.

- Door thresholds. Whenever the cart passes a door threshold, the threshold switch attached to the cart will actuate. Since the PLC should know the approximate vicinity of the cart, it can use the door threshold signal to correct its cart position variable to the actual distance of the door it is passing. The reported distance will be from the home position to the cart's limit switch. If the cart is reversed while the limit switch is on a threshold, the correction will still occur but it will be off by the length of the threshold. 


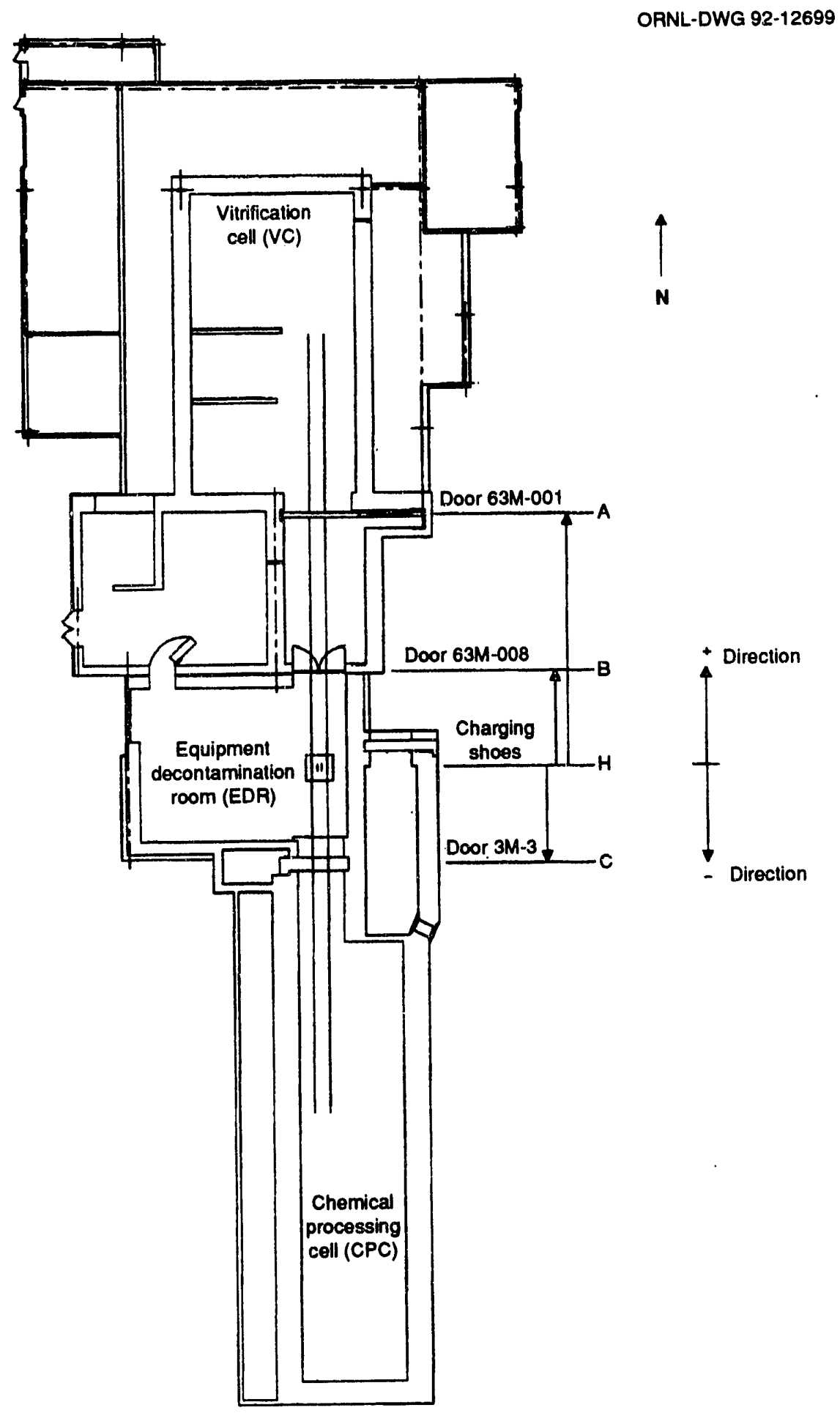

Fig. 9.1. Cart distance definitions for position algorithm. 


\subsubsection{Driving toward unopen door alarm}

The cart control system is required to notify the operator when the cart is approaching an unopen door. The cart position algorithm is used to perform this task, but it reports the distance of the limit switch from the charging shoes. Therefore, it is necessary to compensate the cart posiiion for the distance between the limit switch and either the front or back of the cart, depending on which direction the cart is headed. The compensation must also be based on the orientation of the cart. Except for when the cart is driving southward to doorway $63 \mathrm{M}-0.08$, all alarm distances will be the same ( $-4 \mathrm{ft}$ from the door). However, when driving southward to doorway $63 \mathrm{M}-008$, the alarm distance must be increased to $\sim 10 \mathrm{ft}$ from the door since these doors will swing toward the cart. Therefore, it is necessary for the PLC to determine to which door and in which direction the cart is approaching.

\subsubsection{Battery State of Charge}

Three cart battery parameters are used to determine the battery state of charge: (1) voltage, (2) current, and (3) temperature. Energy removed from the battery in a given time period can be determined by multiplying battery voltage by battery current. Testing of the batteries specified for the cart system has shown that at $20^{\circ} \mathrm{F}$ the batteries can provide $-2.18 \mathrm{MJ}$ of energy before reaching the low-voltage cut-off level. Using this number as the starting value, all subsequent cnergy usage values are subtracted from this initial value. The energy provided by the batteries as a function of temperature follows the Arrhenius equation but in our range of temperatures is very linear. Using the energy produced by the tested batteries at room temperature and the energy produced at $20^{\circ} \mathrm{F}$, a factor of $7 \mathrm{~kJ} /{ }^{\circ} \mathrm{F}$ is found. Therefore, the temperature correction factor is added to the instantaneous battery state of charge to obtain the temperature-compensated state of charge.

When the battery is charging, similar calculations can be performed except that energy is added instead of subtracted. However, since the battery has internal losses, more energy will be used charging the battery than what actually appears as the battery's charge level. Because of this, an uncompensated energy level of $2.18 \mathrm{MJ}$ is the maximum that the state-of-charge indication is allowed to read. This level can be reached in two ways. The first method is that the batteries are on charge full time, and the calculation of energy added gradually sums up to this maximum. The second method is that the battery charge current reaches a trickle flow of $\sim 60 \mathrm{~mA}$, indicating full charge. The second method is useful if a new set of batteries are placed in the cart. Because the history of the new batteries is not known to the control system, charging to the point that the charger goes to trickle charge will indicate that the batteries are fully charged.

\subsection{FACILITY COMMUNICATIONS CONTROLLER SOFTWARE}

Refer to flow chart Dwg. Q-6340-160. Tasks performed by the facility communications controller can be split into two categories: (1) battery charger communications and (2) rf transceiver communications. Since these two modes are mutually exclusive, the controller can dedicate itself to one mode at a time. The PLC will switch the facility communications controller between battery charge mode and transceiver mode. The facility communications controller is a Prolog embedded microprocessor system with an Allen-Bradley interface card, Model 7514, which provides an interface to the PLC's remote input/output. Discrete bits as well as 8-bit bytes containing raw, unscaled analog data will be passed between the Prolog controller and the PLC. 


\subsubsection{Battery Charger Communications}

After every cycle of the cart, the cart will retum to the charging shoes to recharge the cart batteries. The cart will remain on float charge until it is needed again. When in the charge mode, cart systems are disabled. The purpose of the facility communications controller in the battery charging mode will be to interface the battery charger to the PLC. The battery charger, Exide Model ERBC 24/30, is equipped with an RS-232 communications line which enables it to pass charging data to the facility communications controller. The communications link is bidirectional in that commands can also be passed to the charger for starting and stopping the charging sequence. To initiate charging, it is necessary to enter the charger's monitor mode. A START command is issued, but it is not acted upon until exiting from the monitor mode. In the way the charger is designed, the charger would normally start and provide status reports at either 15 -min or 1-h intervals. However, in this application, we would like to know whether charger current is flowing immediately to provide feedback to the PLC that charging is active. Therefore, we must re-enter the monitor mode, where we can request charging data immediately. If the reported charging current is above a minimum threshold, we stay in monitor mode and periodically request charging data from the charger and pass it to the PLC. When a valid stop charge command is received from one of the operator's pendants, the communications controller turns off the battery charger and switches to the transceiver mode.

\subsubsection{Transceiver ( $\mathrm{Tx} / \mathrm{Rx})$ Communications}

In the transceiver mode, the communications controller interfaces to the facility rf transceiver system. At $\sim 0.1-\mathrm{s}$ intervals, the facility if system will transmit to the cart rf system. The commands will originate in the PLC. The protocol and commands are defined in Sect. 9.5. At -1-s intervals, the facility communications controller will pass a command for the cart to return cart data to the facility. The facility rf system will then be switched to receive this data. When the facility communications controller receives the data, it will place it in shared memory with the PLC and retum to the transmit mode.

\subsection{CART CONTROLLER SOFTWARE}

Refer to flow chart Dwg. Q-6340-210. The cart controller software will run on the WinSystems embedded controller. Note that the cart electronics are disconnected when the batteries are charging; therefore, the cart controller will not be powered, and this software will not be running. When the cart is powered up (or after a HARD BOOT command is issued from the facility) the system will be initialized and calibration checks will be performed on the system A/D converters. After initialization, the cart controller will run an endless loop.

The first step in the loop is for the cart controller to read and store system data points. It will then act on the data it receives. For instance, if the cart electronics temperature is high, it will switch on the cart fan.

\subsubsection{Data Packet Status}

The next step is to check for a communications packet from the facility. Three modes are envisioned for cart drive and auxiliary outlet switching based on the status of the data packet. 


\subsubsection{Valid data packet received}

When a valid data packet is received that contains either a cart drive command or an auxiliary outlet enable command, the commands will be acted upon. When a valid data packet is received that does not include a drive command nor outlet enable command, the cart motors and auxiliary outlets will immediately be disabled.

\subsubsection{Bad data packet received}

If a bad data packet is received and the previous data packet did not contain a drive command nor outlet enable command, no drive or outlet will be enabled. However, if a bad packet is received and the previous valid packet included a drive command or outlet enable command, the cart drive or outlet enable will be continued for a short interval. If after this interval bad data packets are still being received, the auxiliary outlets will be disabled. However, if in the cart drive mode, the cart will go into a null location avoidance routine. In this routine, the cart motors will stay enabled for a short period, as discussed later, to continue to drive the cart in an attempt to move out of an rf signal null location. However, the cart will not be allowed to move farther than the maximum stopping distance (6 in.) stated in the project design basis. It is estimated that with the dynamic braking provided by the cart motor controllers, the maximum distance the cart will roll after the motor drive is disabled is 1 in. That distance allows a maximum roll distance of $5 \mathrm{in}$. before issuing a stop command. Then, at the nominal cart speed of $15 \mathrm{ft} / \mathrm{min}$ ( $3 \mathrm{in} . / \mathrm{s}$ ), the maximum time delay before disabling motor drivers is $1.67 \mathrm{~s}$. To ensure that the cart will roll through a signal null location, the cart should be allowed to roll at least the quarter wavelength of the communications system. At the communications carrier frequency of $915 \mathrm{MHz}$, the quarter wavelength is 3.23 in. Therefore, if the cart is allowed to go at least 3.23 in., the minimum delay that should be used in this routine (at the nominal cart speed) is $1.08 \mathrm{~s}$. In summary, the delay provided by the null location avoidance routine should be between 1.08 and $1.67 \mathrm{~s}$. The additional distance moved by using the routine should be enough to drive the cart out of the rf signal null.

\subsubsection{No data packet received}

If no data packet is received and the previous valid data packet did not include a drive command or outlet enable command, no drive command or outlet enable command will be passed to the cart. However, if the previous valid data packet included a cart drive request or outlet enable command, the cart drive or outlet enable will be continued for a short interval. After this interval, if no data packet is received, the cart will stop and the outlets will be disabled. The duration of the interval is expected to be $-0.3 \mathrm{~s}$, which should stop the cart well within the maximum stopping distance requirement.

Finally, the cart controller will check for a report status command from the facility. If this command is received, the cart controller will switch the rf system to transmit, transmit the status packet, and return to the receive mode.

\subsection{COMMUNICATIONS PROTOCOL AND COMMAND DEFINITIONS}

The transmission of data and commands between the transfer cart and the facility control system requires additional processing over that provided by the control system biphase encoder/decoder hardware. This additional processing is performed in software by the transmitting and receiving microprocessor systems and made possible by a communications protocol. A communications protocol is a technique for organizing bytes of binary data into a structured data packet for transmission and reception. Using a protocol provides the software mechanism for 
detecting communication errors in addition to the hardware error detection for tagging data packets as intended for a particular receiver and transmitting raw data (e.g., such as might be read from A/D converters). This results in significantly reducing the risk of the raw data causing decoding errors at the receiving end.

\subsubsection{Reasons for Using a Communications Protocol}

Since there can be two cart communication systems in operation simultaneously (one operating and one on standby) it is necessary to provide a means for each individual cart electronic system to determine if a received command is intended for it. The communications protocol has an ID byte reserved for this purpose. When a cart electronic system receives a command that requires transmission of data back to the facility computer, the system's hardware-jumpered ID number is read and compared to the ID contained in the received data packet. If the two IDs compare, then the command will be performed; otherwise, the command is ignored. Since it is conceivable that the electronic hardware that provides the local cart system ID could fail, only commands that request transmission of status data are subject to this test. This precaution was taken to reduce the number of items that, when failed, would result in a stranded cart. This scenario works because it does not matter if a cart system on standby performs any of the other commands since it has no motors connected. On the other hand, more than one cart system transmitting simultaneously will cause garbled transmission to the facility.

If the various $A / D$ readings taken by the active cart's internal controller are transmitted back to the facility without the using a protocol, there is a high probability that the cart system on standby will interpret the data as a valid ID followed by a valid command. This could cause the standby system to start transmitting data while the on-line cart system is transmitting. The communications protocol places the ID byte in a predetermined position in the transmitted packet and frames the data bytes with predetermined values. This makes it possible for the receiving end to perform tests on packets to greatly reduce the probability of false decoding.

A third justification for using a communications protocol is that the number of bytes in each transmitted packet can be included as information in the packet. The CRC circuit used in the hardware to test for communication errors requires 16-bit data for correct operation. If an odd number of bytes are transmitted, the CRC circuit at the transmit end will append a null data byte to the end of the packet to produce a 16-bit value. The number of bytes sent as information in the transmitted packet enables the receiving end to detect these null bytes and strip them off.

\subsubsection{Description of the Communications Protocol}

The communications protocol for the transfer cart is described below. Under this protocol, all data and command bytes will be transmitted in a packet format using the following structure.

\subsubsection{Transmitted data}

A graphical representation of a transmitted packet is shown below with the first byte starting

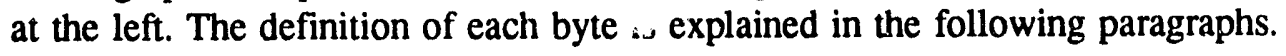

\begin{tabular}{|c|c|c|c|c|c|c|c|}
\hline NCHAR & STX & STX & ID & DATA $_{1}-$ DATA $_{(\text {NCHAR - 3) }}$ & ETX & ETX & CKSUM \\
\hline
\end{tabular}


Number of characters. The first byte transmitted will be the number of bytes in the packet represented as an 8-bit binary number. This byte field will be referred to as the mnemonic NCHAR. The byte count is the actual number of bytes transmitted including NCHAR and CKSUM.

Head of data frame. The second and third bytes will always be the ASCII code for the start of the text and will be referred to as the mnemonic STX.

ID byte. The fourth byte is designated as the identification byte and will reflect the intended receiver of the packet. This byte is referred to as the mnemonic ID. Five ID bytes are defined in the following table.

Table 9.1. Cart ID byte definitions

\begin{tabular}{cccc}
\hline ASCII & Hex & Binary & Description \\
\hline$*$ & $0 \times 2 A$ & 00101010 & Cart 1 ID character \\
U & $0 \times 55$ & 01010101 & Cart 2 ID character \\
$\%$ & $0 \times 25$ & 00101001 & Cart 3 ID character \\
p & $0 \times 70$ & 01110000 & Cart 4 ID character \\
f & $0 \times 66$ & 01100110 & Facility ID character \\
\hline
\end{tabular}

Data fields. The next field of the packet is the data field where the transmitted data or commands are stored. This is a variable length field and can range from 1 to 41 bytes long. The maximum number of bytes contained in a transmitted packet must not exceed 48 because the hardware FIFO buffers used in both the transmit and receive circuits are 48 bytes deep. The packet protocol has an overhead of 7 bytes, leaving a maximum of 41 bytes available for data or commands. In the case of transmitting multiple commands to the cart, the ordering of the commands in the data field determines the priority of execution (i.e., the commands are executed in the order they are received, starting with the first data byte.)

Tail of data frame. Byte position NCHAR - 2 and NCHAR - 1 will always be the ASCII code for end of text referred to as the mnemonic ETX.

Check sum. Byte position NCHAR will always be the check sum of all the previous bytes and will be referred to as the mnemonic CKSUM. The check sum is calculated by simply adding each byte to an initial 8-bit value of 0 and ignoring overflows. The receiving end performs the identical operation on the received packet and compares the answer to the transmitted check sum.

\subsubsection{Received data}

The following steps can be applied to test a received data packet for errors. Steps 1 and 2 are mandatory. Step 3 can be used for an even higher level of confidence that a good packet has been received without data corruption. 
Number of characters. First, test to determine the number of characters sent (NCHAR) is an odd or even number. Because the biphase hardware uses 16-bit technology to perform the CRC on transmitted and received data, the board will add a null byte to the end of the packet if an odd number of bytes is transmitted by the biphase board. This null byte will cause the receiving process to receive one more byte than was in the original packet. If NCHAR is odd and the actual number of characters received is NCHAR +1 , then subtract 1 from the number of characters received and assume the correct number was received. If the number of characters received (NCHAR) is an even number, then NCHAR should equal the number received.

Check sum. Calculate the check sum of the received data packet by doing a modulo 256 sum of all the bytes in the packet except the last byte (CKSUM). This sum must equal the CKSUM value in the last byte if the data packet is to be considered a good packet.

Data frame. To help ensure against the unlikely occurrence of a compounded data corruption which results in a correct CKSUM calculation even though an error has occurred, test that the data frame is correct. The second and third bytes in the packet must be equal to STX, and the bytes at NCHAR - 1 and NCHAR - 2 must be equal to ETX.

\subsubsection{Exception to Communications Protocol}

An exception to the packet protocol occurs when a HARD BOOT command must be sent to the cart from the facility. If the cart's microprocessor control system becomes locked in a nonresponsive state, it will not execute commands sent from the facility. To recover from this condition, the receiver portion of the digital communications circuit is equipped with a hardware reset function. This function will reset the microprocessor system by forcing the reset line low on the computer bus. This feature is implemented in the receiver hardware by comparing for two consecutive occurrences of a predefined 8-bit pattern in the incoming byte stream. This pattem must occur on an odd/even byte boundary, and the comparison is made only on the incoming data to the cart and not on outgoing data nor does the facility receiver have this feature. Because of this, raw data being transmitted from the cart will not trigger false hardware resets. The HARD BOOT command is executed by sending a minimum of six pairs of the reset code, starting with the first byte. The reset will probably occur on the first or second pair, but six pairs are sent to ensure that the reset code is detected. The extra pairs are of no consequence since the receiver hardware is also reset, which results in the additional pairs being cleared out. The reset code has been defined as the hexadecimal value F0. The 8-bit binary pattern for this value is 11110000 . The following diagram clepicts the HARD BOOT data packet. The dip switch on the cart biphase encoder/decoder, which determines this reset code, should be set to this value (See Sect. 6.7.3.9).

\begin{tabular}{|l|l|l|l|l|l|l|l|l|l|l|l|}
\hline F0 & F0 & F0 & F0 & F0 & F0 & F0 & F0 & F0 & F0 & F0 & F0 \\
\hline
\end{tabular}

\subsubsection{Facility Commands to Cart}

Table 9.2 defines the commands the facility can send to the cart. 
Table 9.2. Facility commands to the cart

\begin{tabular}{ccl}
\hline $\begin{array}{c}\text { CMD } \\
\text { char }\end{array}$ & $\begin{array}{c}\text { Hex } \\
\text { value }\end{array}$ & \multicolumn{1}{c}{ Description of command } \\
\hline$>$ & 3E & Drive forward \\
$<$ & $3 \mathrm{C}$ & Drive backward \\
$\mathrm{X}$ & 58 & Report status \\
$\mathrm{A}$ & 41 & Reset Motor 1 \\
$\mathrm{B}$ & 42 & Reset Motor 2 \\
$\mathrm{C}$ & 43 & Reset Motor 3 \\
$\mathrm{D}$ & 44 & Reset Motor 4 \\
0 & 30 & Enable Outlet 1 \\
1 & 31 & Enable Outlet 2 \\
$\mathrm{N}$ & 4E & Null packet \\
+ & 2B & Increase speed \\
- & 2D & Decrease speed \\
$\mathrm{T}$ & 54 & Acknowledge door threshold \\
\hline
\end{tabular}

The Drive forward, Drive backward, Enable Outlet 1, and Enable Outlet 2 commands must be sent repeatedly to remain active. If one of these commands is in effect and three consecutive valid transmissions are received without the command present or if communications to the cart stops for more than $0.3 \mathrm{~s}$, the command will be canceled. The other commands are executed only once when received and must be retransmitted before they will be executed again. The Increase speed and the Decrease speed commands increase or decrease the cart speed by a small amount each time they are executed. This speed change will remain in effect until changed by another speed change command or until the cart is reset or powered off.

The Increase speed and Decrease speed commands are not system requirements for this project but have been added for convenience. We anticipate that the pulse-width-modulated signal used for motor speed control will require modifications to obtain the desired nominal cart speed of $15 \mathrm{ft} / \mathrm{min}$. Without the capability to change this speed from the engineer's console, the programmable read-only memory (PROM) of the cart control system would have to be reprogrammed each time a new speed set point is tried-an exercise that may take several iterations during cold testing. By having this capability, the speed set point can be adjusted to the appropriate level remotely during cold testing and the final value then programmed into the cart PROM only once. At that time, if the ability to increase or decrease speed is no longer needed, it can be removed from the software (no hardware modification is required in either case). 
Below is a graphical representation of a communication packet sent to Cart 1 commanding continued forward motion, reset Motor 2, and requesting a cart status report. The first row is the packet in mnemonic form, and the second row contains the actual hexadecimal values sent in the correct byte order.

\begin{tabular}{|c|c|c|c|c|c|c|c|c|c|}
\hline NCHAR & STX & STX & $*$ & $>$ & B & X & ETX & ETX & CKSUM \\
\hline OA & 02 & 02 & $2 A$ & $3 E$ & 42 & 58 & 03 & 03 & 16 \\
\hline
\end{tabular}

\subsubsection{Cart System Status Report}

Table 9.3 defines the retumed data packet from the cart after the facility has requested a status report. Byte No. 22, status bit flags, contains information based on the state of the individual bits. These bits flags are defined in Table 9.4, where LSBit and MSBit refer to least significant and most significant bits respectively. 
Table 9.3. Cart system status report

\begin{tabular}{|c|c|c|}
\hline $\begin{array}{l}\text { Byte } \\
\text { No. }\end{array}$ & Contents & Description \\
\hline 1 & NCHAR & Number of bytes in packet \\
\hline 2 & STX & ASCII start of text $(0 \times 02)$ \\
\hline 3 & STX & ASCII start of text $(0 \times 02)$ \\
\hline 4 & ID & Facility ID (ASCII character f) \\
\hline 5 & DATA1 & Motor 1 voltage \\
\hline 6 & DATA2 & Motor 2 voltage \\
\hline 7 & DATA3 & Motor 3 voltage \\
\hline 8 & DATA4 & Motor 4 voltage \\
\hline 9 & DATA5 & Motor 1 current \\
\hline 10 & DATA6 & Motor 2 current \\
\hline 11 & DATA7 & Motor 3 current \\
\hline 12 & DATA8 & Motor 4 current \\
\hline 13 & DATA9 & Battery voltage \\
\hline 14 & DATA 10 & Battery current \\
\hline 15 & DATA11 & Battery temperature \\
\hline 15 & DATA12 & Outlet 1 voltage \\
\hline 17 & DATA13 & Outlet 2 voltage \\
\hline 18 & DATA14 & Outlet 1 current \\
\hline 19 & DATA15 & Outlet 2 current \\
\hline 20 & DATA16 & Electronics enclosure temperature \\
\hline 21 & DATA17 & Percent usage of Antenna 1 \\
\hline 22 & DATA 18 & Status bit flags \\
\hline 23 & DATA19 & ID of sender $(*, U, \%$, or $p)$ \\
\hline 24 & ETX & ASCII end of text $(0 \times 03)$ \\
\hline 25 & ETX & ASCII end of text $(0 \times 03)$ \\
\hline 26 & CKSUM & Modulo 256 sum of bytes 1 to 25 \\
\hline
\end{tabular}


Table 9.4. Cart status bit flags

\begin{tabular}{cl}
\hline $\begin{array}{c}\text { Bit } \\
\text { position }\end{array}$ & \multicolumn{1}{c}{ Definition } \\
\hline LSBit & Door threshold detect \\
1 & Motor 1 overcurrent trip \\
2 & Motor 2 overcurrent trip \\
3 & Motor 3 overcurrent trip \\
4 & Motor 4 overcurrent trip \\
5 & Outlet 1 overcurrent trip \\
6 & Outlet 2 overcurrent trip \\
MSBit & Calibration shunt error \\
\hline
\end{tabular}




\section{SUMMARY AND CONSIDERATIONS}

This report documents the design of a control system for the West Valley transfer cart at the time detailed hardware design and prototype testing are complete. Software has been developed to the stage that it was used for integrated hardware testing, but more development will be required to bring it to the level of being ready for the entire control system and to work out some problems that surfaced during integrated hardware testing. The results of the hardware testing indicate that the system should operate as designed. However, minor modifications will almost surely be necessary when the control system is tested and installed at the West Valley site.

All design and operating personnel should be aware of subtleties in the control system design that may or may not present a problem at some phase of operation. Some of these points were discussed in design review meetings and are included herein only for formal documentation. Other points also developed during integrated testing. The considerations are listed somewhat in order of priority.

1. Absence of cart stop switch. The transfer cart was designed with only two cart modes: run and battery charge. If the cart is abandoned for a long period of time, its battery will discharge and it will be unable to drive itself back to the battery charger.

2. Facility antenna design. There are tradeoffs to be made between the rf characteristics, the flexibility, and the rad hardness of the push-through rods for the facility anteinas. Some if measurements have been performed in a laboratory that show that the first choice for materials and cables did not have sufficient if characteristics (there were many reflections from a design with an aluminum base). Other materials and cables have been specified in the final design, but time did not allow for the fabrication and testing of the system. More time may be needed either in the lab or at the facility to verify the optimum antenna system.

3. Engineer's console data retention. If the control system does not receive new incoming data from the cart, the memory will retain old values of variables. This could be confusing in that it may make it appear that the cart is still operating normally when it fails to transmit new data. It may be necessary to zero the values when no new data is received to avoid this confusion. This condition also occurs when the cart ID is changed and no other cart system is active.

4. Cart diversity antenna minimum switching rate. Because switching logic of the cart antennas is triggered on the rising edge of the first facility carrier that occurs after the cart transmitter has shut off, the cart reception signal cannot be switched between cart antennas any faster than the carrier pulse rate of the facility rf system. At present, the facility carrier stays active for almost $1 \mathrm{~s}$, and then switches off to allow reception of the cart status signal. Therefore, although command messages are sent to the cart every $0.1 \mathrm{~s}$, the cart is unable to switch between its receiving antennas any faster than once every $1 \mathrm{~s}$. Given the many design features 
to prevent a signal null from stopping the cart, this does not present a major problem at this time.

5. Emergency stop functionality. Because the emergency stop function eliminates communications with the cart, requests for cart data cannot be sent. Therefore, during an emergency stop condition, cart status is no longer known. Only two operating modes are provided for the cart: battery charging and cart operation. It is not desirable to kill the cart since, then, there would be no way to restart it remotely. When emergency stopped, the cart will be in an idle mode, not completely dead.

6. Door interfacing fail safety. When a door is energized, it prevents all cart operation. Fail-safe circuitry of the door-energized inputs should be addressed to ensure that a failed door energized status will prevent cart operation. Limit switch circuits which indicate an open door should be addressed in a similar manner, as they are used to warn when the cart is approaching an unopen door.

7. Engineer's console heat load. No formal heat loading analysis was performed on the engineer's console. The cabinet contains much heat-producing equipment. A fan was specified for cooling, but its effect at keeping the cabinet below reasonable temperatures for the electronic equipment is not known.

8. Cart reboot command. The cart reboot command will reboot any electronics rack that is online. For example, if communication to a spare electronics rack is desired and a cart reboot command is given, the cart reboot command will also reboot the operating cart electronics rack.

9. Facility antenna disable function. If a facility antenna is disabled, the engineer must remember to reenable it later - there is no alarm or flag to remind him to do so. If it is always necessary to disable an antenna when the cart is at a certain position, the PLC could be programmed to disable and then reenable the antenna.

10. Engineer's console trackball. Because the engineer's console software is Windows based, it would be more operator efficient to have a trackball as a pointing device. However, as configured, no trackball was specified for the system. All of the Windows features should still be accessible through keystrokes, but if after the unit is installed it becomes obvious that a trackball is needed, options should be investigated for interfacing a trackball to the unit. Since two serial ports are already being used on the computer, a bus trackball should be specified.

11. Cart speed control. Although the cart speed can be changed at the engineer's console, the speed is not retained by the cart microprocessor after a cart reboot. After cold testing, the nominal cart speed should be programmed into the cart microprocessor PROM so that it is the default after booting up. 


\section{REFERENCES}

1. T. W. Burgess et al., Summary Report of CFRP Experiences with Signal Transmission Techniques for Remote Applications, ORNL/TM-10599, Martin Marietta Energy Systems, Oak Ridge Natl. Lab., February 1988.

2. R. E. Ziemer and W. H. Tranter, Principles of Communications, 2 ed., Houghton Mifflin Co., Boston, 1985.

3. Instrumentation Symbols and Identification, ISA-S4.1, rev. 3, Instrum. Society of America, Research Triangle Park, N.C., 1984. 


\title{
APPENDIX A \\ ANALYSIS CF THE SHIELDING DESIGN FOR THE WEST \\ VALLEY FACILITY VITRIFICATION CART
}

\author{
C. O. Slater \\ OK: L Engineering Physics and Mathematics Division
}

May 31, 1991

\section{INTRODUCTION}

In an April 1991 meeting, a proposed shielding design for the West Valley Vitrification Facility cart was discussed. The shielding thickness was determined using a handbook estumate of the attenuation of the dominant gamma-ray source. Following a modification to the initial shielding design, detailed calculations were performed to verify the adequacy of the shielding design. Results of the analysis are presented.

\section{SHIELDING DESIGN}

The modified shielding design is shown in Fig. A.1. Important dimerizions are noted on the figure. The main areas of concem are the shield openings where radiation may stream into the cavity housing electronics and batteries. Section B-B shows the top opening and Section C-C shows a lower side opening. Dimensions for each opening are given in Sections C-C and B-B, respectively. The cavity dimensions are shown in Section A-A. The shielding material is steel-encased lead.

\section{RADIATION SOURCE}

The background radiation source is abciut $10^{3} \mathrm{rad} / \mathrm{hr}$ and is due to waste in the canisters. The radionuclide inventory in an average canister in 1990 is shown in Table 1. The source for the shielding calculations was obtained first by inputting the Curie inventories of the major isotopes (those with greater than 200 Curies) into the C.IIGEN/S computer code to calculate the gamma-ray spectrum in the 18-group energy structure used in the shielding calculations. Next, the ORIGEN gamma-ray source was used as input for a 1-D cylindrical ANISN calculation for one of the canisters, which is described in Fig. A.2. The 1-D ANISN cylindrical surface flux was then adjusted using dose conversion factors to give $10^{3} \mathrm{rad} / \mathrm{hr}$ on the surface of the cart. An isotropic angular flux was assumed. Table 2 shows the energy group structure, ORIGEN spectrum, the ANISN surface flux, the dose conversion factors, and the surface flux (with the ANISN spectrum) that gives a $10^{3} \mathrm{rad}-\mathrm{Si} / \mathrm{hr}$ dose rate. 


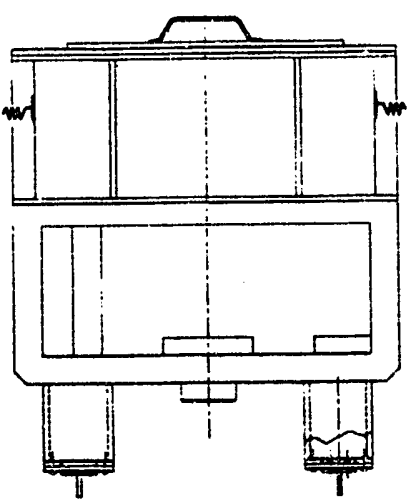

View D-D

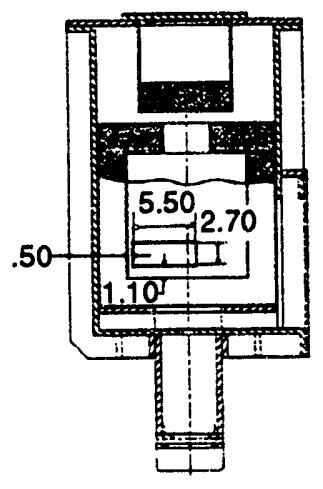

Section B - B

Note: Dimensions in inches
ORNL-DWG 92-12726
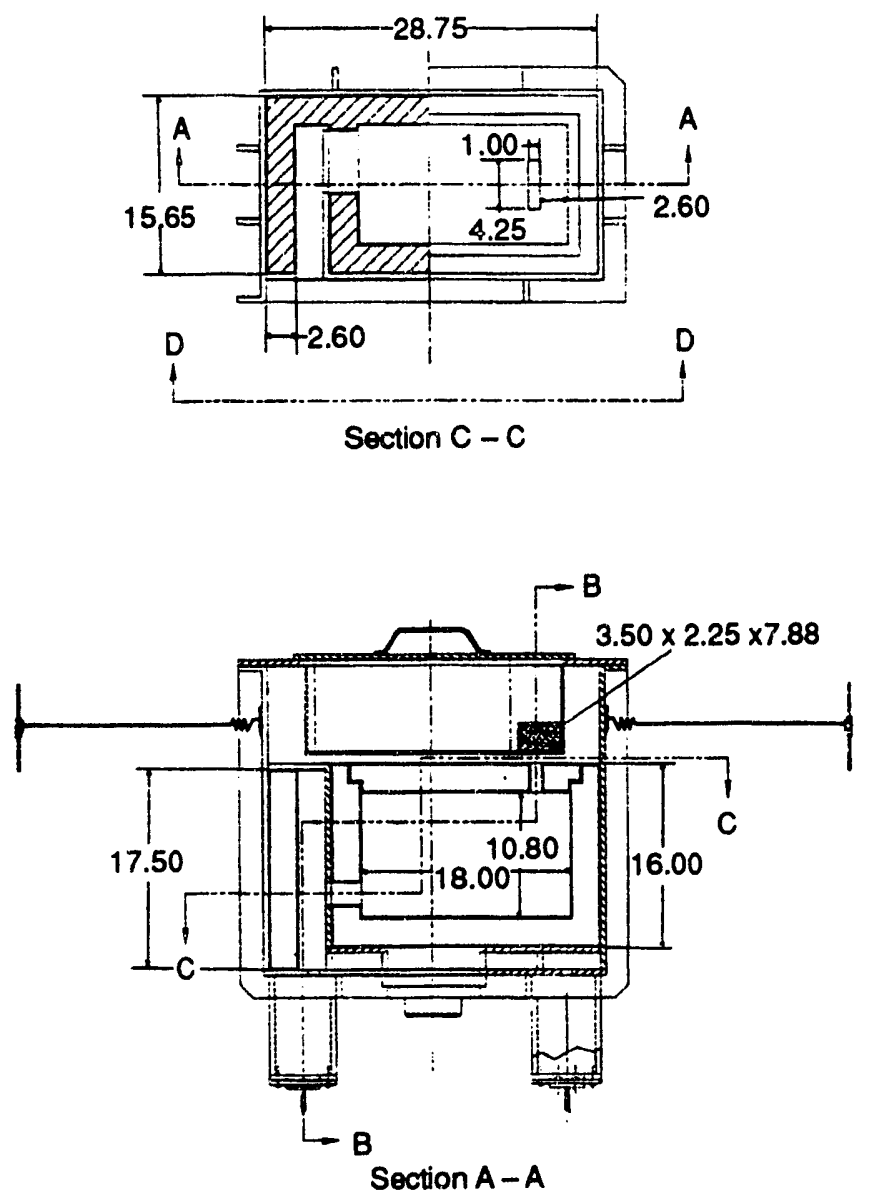

Fig. A.1. Proposed shielding design for the West Valley Vitrification Facility cart. 
Table 1. Radionuclide inventory for an average West Valley Nuclear Services canister for the year 1990

Isotope

3-H

14-C

55- $\mathrm{Fe}$

59- $\mathrm{Ni}$

63-Ni

$60-\mathrm{Co}$

79-Se

90-Sr

$90-Y$

93-Zs

$93 \mathrm{~m}-\mathrm{Nb}$

99-Tc

106-Ru

106-Rh

$107-\mathrm{Pd}$

125-Sb

$125 \mathrm{~m}-\mathrm{Te}$

126-Sn

$126 \mathrm{~m}-\mathrm{Sb}$

126-Sb

129-I

134-Cs

135-Cs

137-Cs

$137 \mathrm{~m}-\mathrm{B}$

144-Ce

144-Pr

147-Pm

151.Sm

152. Eu

154-Eu

155-Eu

232-Th

233-U

234-U

235.U

236-U

237-Np

238-U

238-Py

239-Np

239-Pu

240-Pu

241-Pu

241-Am

242-Pu

242-Am

$242 \mathrm{~m} \cdot \mathrm{Am}$

242-Cm

243-Am

243- $\mathrm{Cm}$

244-Cm

$245-\mathrm{Cm}$

246-Cm
Activity (Curies)

0.00
0.00
2.76
0.42
30.18
3.03
0.01
$26,330.25$
$26,337.24$
1.07
0.72
0.43
0.06
0.06
0.04
28.55
7.00
0.41
0.41
0.06
0.00
20.33
0.63
$28,341.24$
$26,811.19$
0.00
0.00
345.02
331.40
1.43
375.11
93.68
0.01
0.04
0.02
0.00
0.00
0.09
0.00
32.58
1.36
6.39
0.00
4.68
316.95
209.91
0.01
1.16
1.17
0.96
1.36
0.53




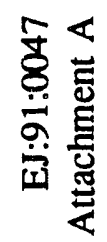
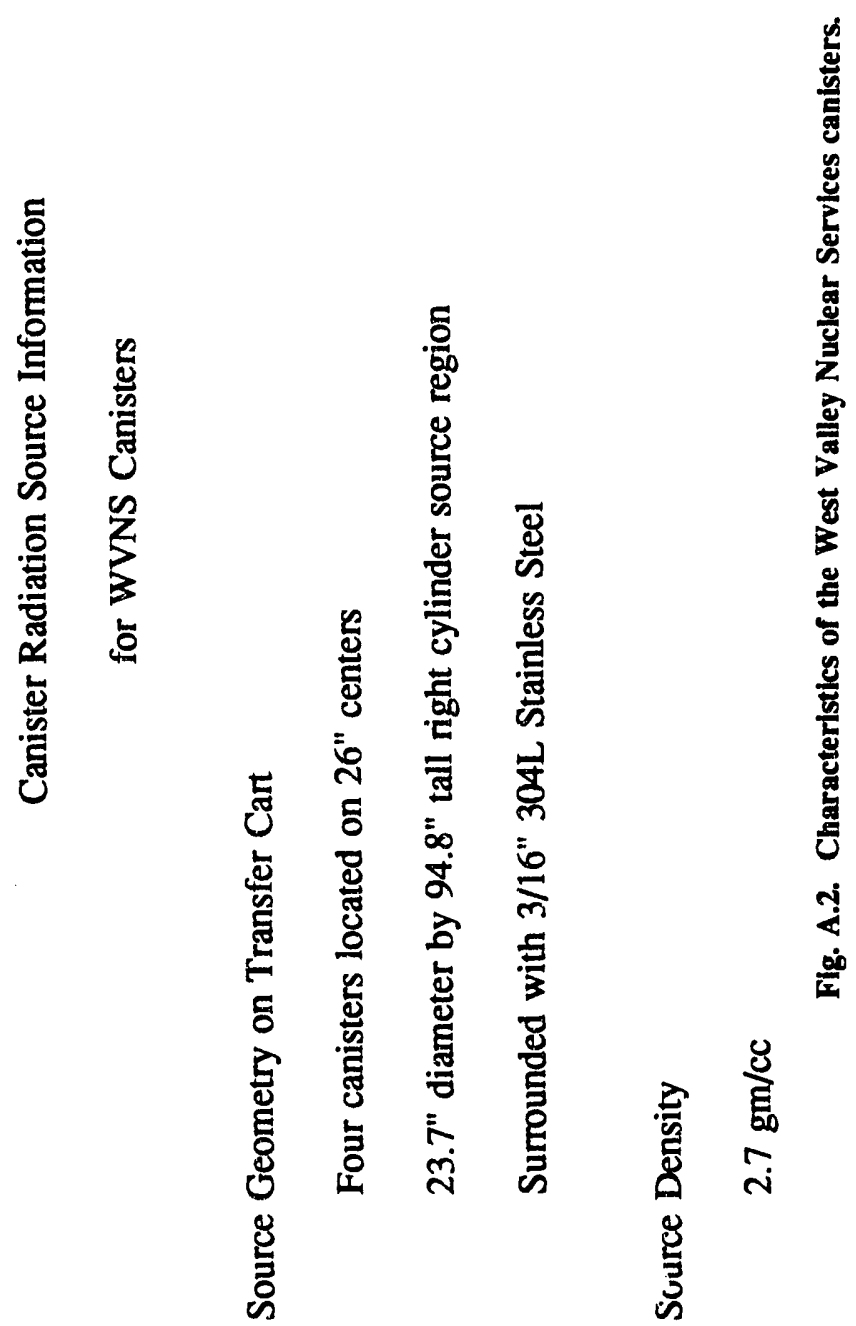


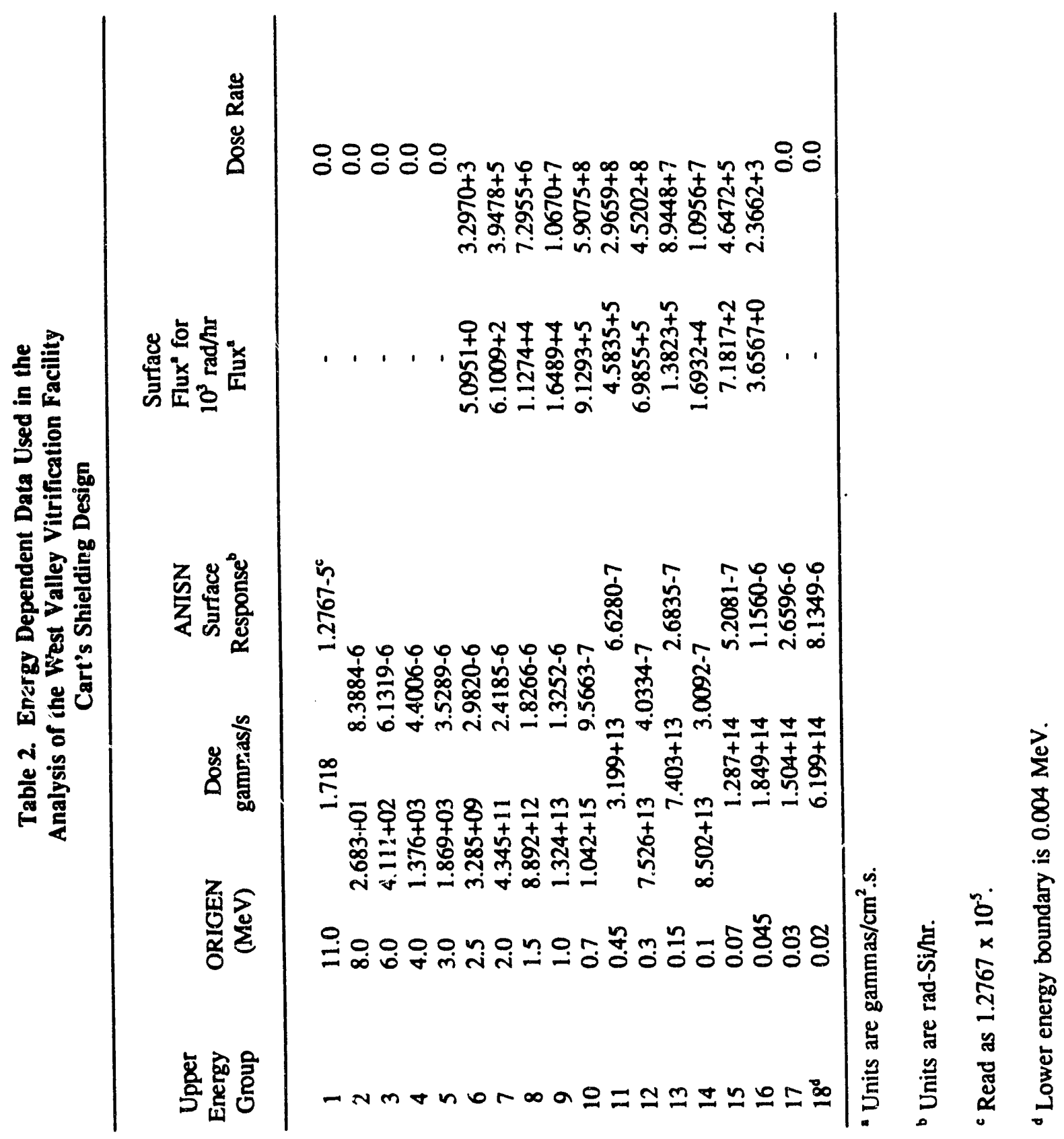




\section{CALCULATIONS AND RESULTS}

Two DORT 2-D X-Y discrete ordinates calculations were performed to confirm the adequacy of the shield design. A calculation using an elevation view of the cart (Section B-B of Fig. A.1) was performed to assess gamma-ray streaming through the top opening of the shield to the cavity. Similarly, a calculation based on a plan view of the cart (Section C-C of Fig. A.1) assessed gamma-ray streaming through the lower side opening of the shield to the cavity. The calculations are conservative in that the openings through the shield are infinite in the third dimension. Such openings should permit more gamma rays to stream into the cavity than would enter if the third dimension of the opening were finite.

Results of the calculations are shown in Figs. A.3 and A.4. The figures show isodose rate contours in the shield and within the cavity. As mentioned earlier, the calculations used an isotropic angular flux as a surface source. The flux had been adjusted to give a $10^{3} \mathrm{rad} / \mathrm{hr}$ dose rate at the surface. However, since only half of the flux was directed into the cart, the figures show about $500 \mathrm{rad} / \mathrm{hr}$ dose rate at the surface of the cart. The important result is the reduction of the dose rate by the shield. For the top opening, Fig. A. 3 shows about a factor of 500 reduction, while Fig. A.4 shows slightly greater than a factor of 1000 reduction for the side opening. Although the calculation for the top opening does not give the desired factor of 1000 reduction within all of the cavity, such a reduction can be achieved by locating sensitive equipment at least $6 \mathrm{~cm}$ from the top of the cavity. In both cases the dose reduction is at least a factor of 1000 relative to the background dose rate of $1000 \mathrm{rad}-\mathrm{Si} / \mathrm{hr}$. Thus, the shielding design appears to be adequate.

\section{SUMMARY}

Conservative DORT 2-D X-Y discrete ordinates calculations were performed for the West Valley Vitrification Facility cart. A goal of a factor of 1000 reduction in the dose rate in the cavity relative to background appears to have been achieved by the proposed shielding design. More detailed confirmatory calculations should not be necessary. 


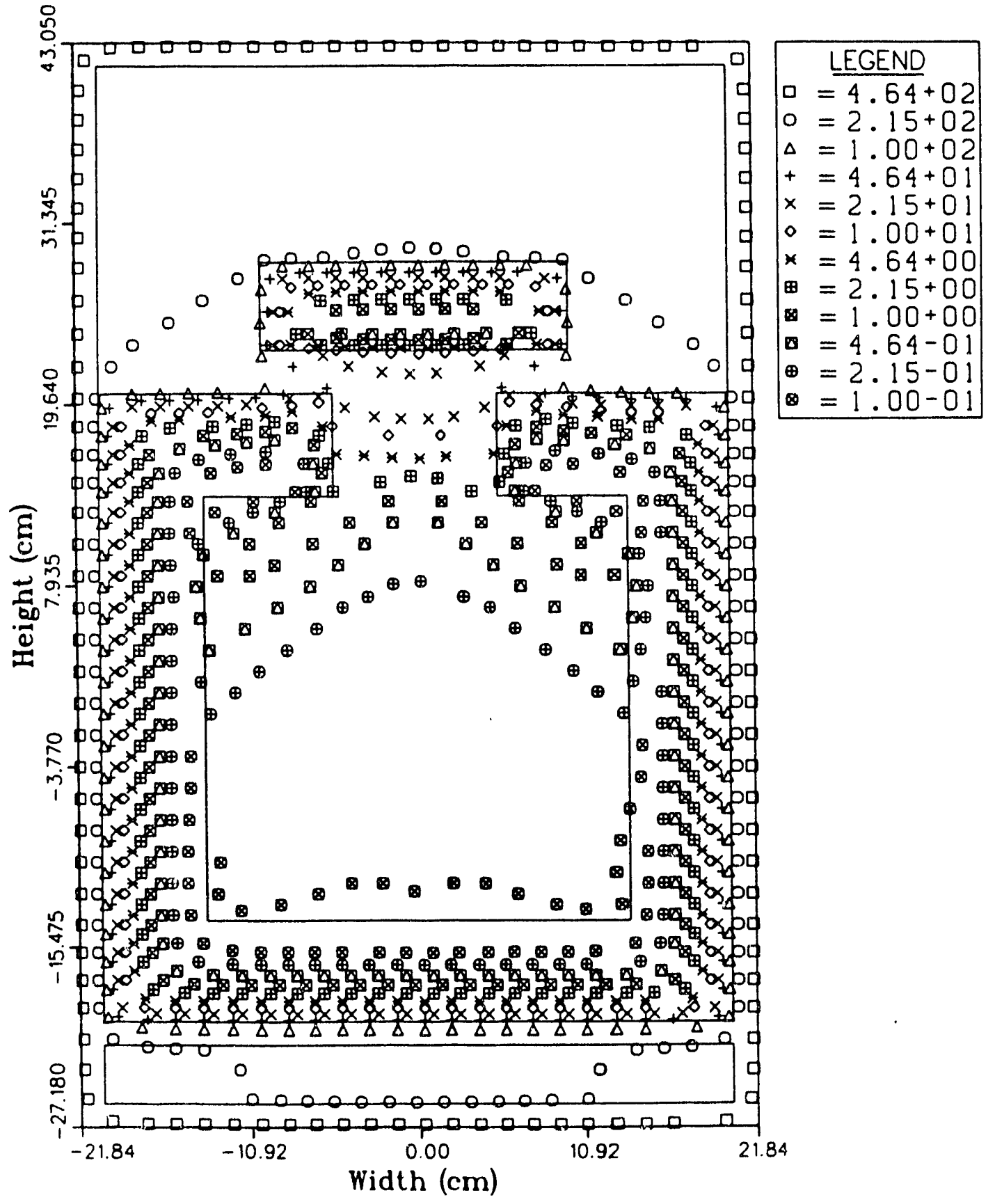

Fig. A.3. Gamma-ray isodose rate contours (rad-SI/hr) for an Vitrification Facility cart (Sect. B-B of Fig. A.1). 


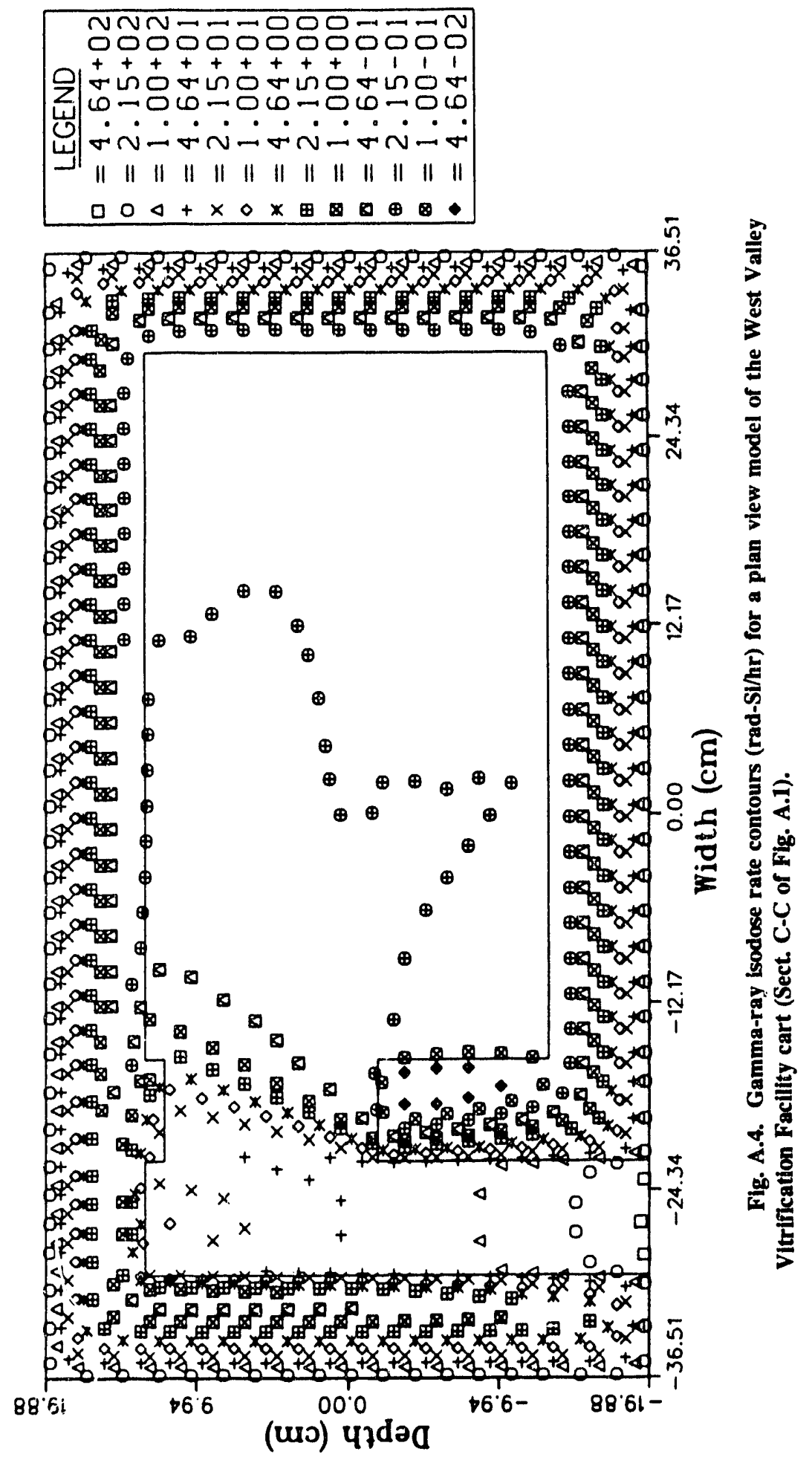




\section{APPENDIX B RADIATION TEST RE_ORT}

\section{INTRODUCTION}

The purpose of the radiation testing that was performed at Argonne National Laboratory (ANL) during the week of September 9, 1991, was to test certain electronic components that will be used in the West Valley transfer cart project. During the five-year design life-time of the cart, the electronics in their shielded enclosure are expected to see a total integrated dose (TID) of about $10 \mathrm{krad}$. Certain components (mainly metal-oxide semiconductor transistor-based electronic devices) that will be used in the cart electronics have radiation withstand capabilities in this same order of magnitude, and thus further evaluation of their radiation tolerance was warranted. Results of the testing indicate a high probability that the cart electronics will survive their five-year design life.

\section{EQUIPMENT IRRADIATED}

Equipment involved in the test can be divided into three categories. The first category was designated test equipment and includes equipment not irradiated, but used to monitor the function of other equipment that was irradiated. The second category is equipment designated as operational irradiated equipment. This equipment was energized and monitored during irradiation. The third category, non-operational irradiated equipment, identifies the equipment to be irradiated in a de-energizes state; i.e., equipment to be neither functioning nor monitored during irradiation.

Operational irradiated equipment was exposed to a steady gamma field of approximately $10 \mathrm{krad}$ per hour. Test plans called for this equipment to be exposed to $100 \mathrm{krad}$ total integrated dose (TID) or until failure of the equipment, whichever occurred first. Proper equipment locations and shielding for the desired exposure rates were detenuined by ANL personnel who made dosimeter measurements prior to the equipment being irradiated.

Table B.1 contains a list of the operational irradiated equipment. Both electronic assemblies from WinSystems failed before the $100 \mathrm{krad}$ TID was obtained. During the exposure period, the functionality of the assembly was automatically monitored and logged by test equipment external to the radiation field. From the time stamp on the logging record, the TID which failure occurred was determined. 
Table B.1 Operational Irradiated Equipment

\begin{tabular}{|c|c|c|}
\hline Description & Manufacturer & Serial No. \\
\hline Single Board Computer & $\begin{array}{l}\text { Winsystems LPM-SBC40R-8 with } \\
\text { ROM-DOS System }\end{array}$ & 1032046 \\
\hline $\begin{array}{l}\text { Analog-to Digital Converter (ADC) } \\
\text { Board }\end{array}$ & Winsystems LPM-AIO & 71572 \\
\hline Power Rack & Winsystems CC8-WM-PS100 & 1012821 \\
\hline SBIO 48 Digital VO & $\begin{array}{l}\text { Winsystems SBX-PIO } \\
\text { SBC Piggy-Back Board }\end{array}$ & 0102864 \\
\hline Single Board Computer & $\begin{array}{l}\text { Winsystems LPM-SBC40R-8 with } \\
\text { C-Thru-ROM System }\end{array}$ & 1032046 \\
\hline
\end{tabular}

The non-operational irradiated equipment was exposed to a steady gamma field of approximately $1 \mathrm{krad}$ per hour, for an intended TID of $10 \mathrm{krad}$. Actual exposure for this equipment was $12 \mathrm{krad}$ TID with about a $\pm 20 \%$ measurement uncertainty. These components consisted of several ORNL-designed electronic cards sized to fit on the STD-bus that will be used for the car electronics and several loose integrated circuits (ICs) and electronic devices. Table B.2 contains a description of the non-operational irradiated equipment components. These components were not tested or powered during the exposure period. They were functionally tested at ORNL prior to and after the exposure.

Table B.2 Non-operational Irradiated Equipment.

\begin{tabular}{||l||l|}
\hline Description & Manufacturer/Part Number \\
\hline IC, Bipolar Comparators & LM393 \\
\hline IC, Bipolar Op-Amp & OP-215 \\
\hline IC, Bipolar Op-Amp & AD708 \\
\hline IC, CMOS Power MOS Driver & Maxim, MAX626 \\
\hline Rectifier HexFETS & IRFD9120 \\
\hline Rectifier HexFETS & IRFD120 \\
\hline Prototype Cart Biphase Encoder/Decoder & ORNL \\
\hline
\end{tabular}




\section{TEST SET-UP}

The irradiation was performed at the ANL gamma facility using their Cobalt-60 gamma source. Test equipment used to monitor the electronics was a signal generator, a personal computer used as a data acquisition system (DAS) and data storage, Metrabyte analog and digital input hardware, and associated cabling. Prior to exposing the electronics to radiation, ANL personnel used cobalt glass dosimetry and shielding as required to determine where to position the two Winsystems STD-bus electronics systems in order to receive the desired dose rate of approximately $10 \mathrm{krad}$ per hour. Likewise, cobalt glass dosimetry and shielding were used to determine a suitable location for the non-operational equipment so that it was exposed to a dose rate of approximately $1 \mathrm{krad}$.

Cables were then routed from the control room to the radiation source cell in order to make the necessary electrical connections to the DAS PC, signal generator and the two rack assemblies. This cabling was necessary in order to monitor and log the operation of these assemblies. A CRT monitor, located in the control room with the DAS PC, was used to display real time data to verify that the DAS and rack assembly were operating properly before and during the irradiation testing. All operational irradiated equipment was verified to be operational prior to being exposed to the gamma source.

Figure B.1 is a block diagram depicting the relative location of the test equipment and irradiated equipment in the test cell and in the control room. This diagram also shows the interconnections between the personal computer data acquisition system and the equipment being irradiated.

\section{FUNCTIONAL TESTING AND SETUP OF OPERATIONAL EQUIPMENT}

The test equipment signal generator was set up to operate in a free-running mode. The frequency of the generator output was adjusted to be $\sim 0.001 \mathrm{~Hz}$. The generator output wave form was selected to be sinusoidal with an approximate $4 \mathrm{~V}$ peak-to-peak amplitude and an approximate $2.5 \mathrm{~V}$ dc offset such that the signal ranged from $\sim 0.5-4.5 \mathrm{~V}$ with respect to ground. This signal was sent to the WinSystems ROM-DOS analog input/output board and also to an analog input on the Metrabyte DAS PC analog input board.

During irradiation testing, the ROM-DOS single board computer was loaded with a $\mathrm{C}$ program to control the analog-to-digital converter (ADC) on the WinSystems analog input/output (AIO) card and record the 12-bit equivalent of the signal generator output voltage. It performed this conversion at a rate much greater than the signal generator frequency, making the change from one reading to the next very small. Following each reading from the ADC, the ROM-DOS microprocessor was programmed to drive 12 of its digital outputs on the piggy-back board with the binary coded value of the signal generators analog voltage read by the ADC. These 12 digital outputs were then monitored by a Metrabyte digital input card in the control room and were read by the DAS PC. In this way the binary code on the digital outputs represented the signal generator output voltage in real time except for a slight delay.

The DAS PC would periodically monitor the signal generator output voltage (via the Metrabyte analog input card) and the binary value of the signal generator output sent from the ROM-DOS single board computer (via the Metrabyte digital input card) and log this information to disk at one-minute intervals. Later analysis of the data would be used to determine within a one-minute interval when something failed 


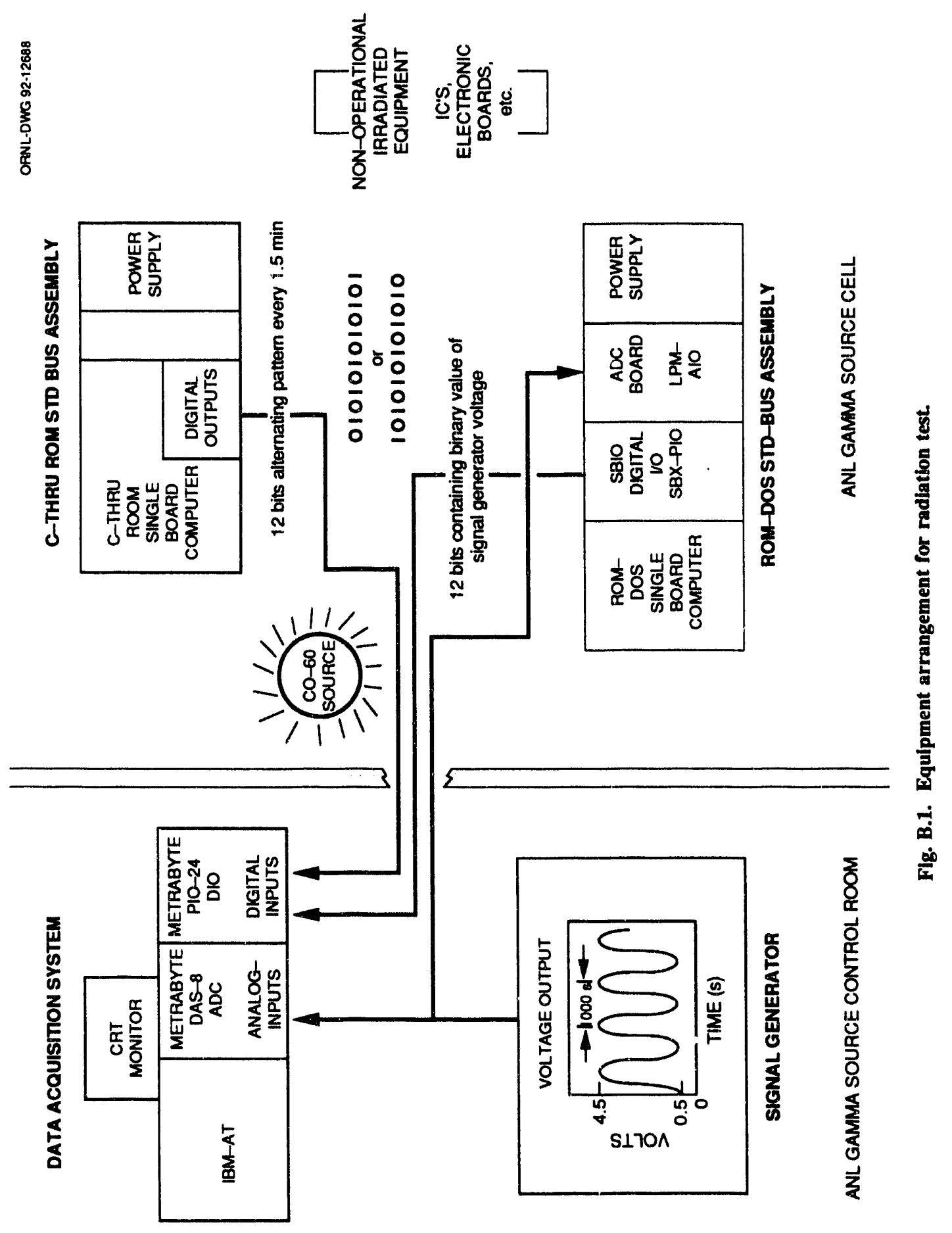


on the ROM-DOS assembly. Failure of the ROM-DOS assembly occurs whenever the logged value of the analog level from the ROM-DOS rack assembly does not match the value read by the DAS within a reasonable tolerance. Failure can be due to rack assembly pov'er supply malfunction, analog board malfunction or microprocessor malfunction.

The second STD rack assembly contained the C-thru ROM single board computer system. This computer system was loaded with a $C$ program to drive 12 of its digital outputs with alternating values of 101010101010 (Decimal: 2730) and 010101010101 (Decimal: 1365). The digital outputs were programmed to change values once every 1.5 minutes. These 12 digital outputs were also sent to the Metrabyte digital input card in the control room via ribbon cable from the source cell to the control room and were monitored by the DAS PC. The DAS PC monitored these digital inputs and logged them to disk at one minute intervals. A discrepancy in the digital outputs or failure to alternate in values would indicate a failure on the C-thru ROM single board computer system.

A CRT monitor was used to display the signal generator output, and the digital outputs from both single board computer systems. This allowed real time monitoring of the single board computer outputs. Upon failure of either single board computer system, irradiation testing was halted to prevent further irradiation of the damaged system. Upon removal of the failed equipment, irradiation testing was then continued.

\section{SETUP AND TESTING OF NON-OPERATIONAL EQUIPMENT}

Prior to irradiation testing, identification, and functional verification testing of individual items to be irradiated as non-operational irradiated equipment was performed and documented at ORNL. For example, regular functional testing results tabulated for each prototype card and chip; curve tracer results for each solid state electronic device; and resistance, capacitance, and various pertinent measurements for each passive device were collected for comparison with these same type of measurements made after the irradiation.

At ANL, the non-operational irradiated equipment cards and electronic devices where positioned and shielded in the exposure area to receive a dose rate of approximately $1 \mathrm{krad}$ per hour. Glass cobalt dosimeters were used to verify the TID.

\section{SUMMARY}

The radiation test on key components of the cart controller system was performed at Argonne National Laboratory. The test went as planned per the Cart Controller Radiation Test Plan, dated September 5, 1991.

The non-operational irradiated equipment which was irradiated in a static mode, i.e. unpowered, survived a total dose of about $12 \mathrm{krad}$, indicated by cobalt glass dosimetry with about a $\pm 20 \%$ measurement uncertainty. Measurements made at ORNL after this testing showed all static equipment survived without measurable affects. 
The two CMOS single board computer assemblies were irradiated while their operation was monitored by the external DAS. These systems were irradiated to failure, presumably caused by radiation degradation. The C-THRU ROM single board computer system, running directly from a compiled C program, failed about 50 minutes into the radiation test, or after a dose of about $9.2 \mathrm{krad}$ with about a $\pm 20 \%$ measurement uncertainty. The other single board computer system (ROM-DOS), similar in hardware design, but with an imbedded IBM DOS firmware operating system, failed after a dose of about $46 \mathrm{krad}(+20 \%$ measurement uncertainty) accumulated in about 4 hours and 50 minutes. In either case, however, the very high dose rate of the test compared to that of the cart service conditions makes the test extremely conservative. Survivability to $9.2 \mathrm{krad}$ in 50 minutes indicates a very high probability of survivability to $10 \mathrm{krad}$ in 5 years.

Both single board computer systems were sent back to WinSystems to allow them to diagnose, identify, and repair the failed systems. Their diagnostics indicated a CMOS RAM chip (Hyundai HY62C256LP10) failed on the C-THRU ROM single board computer. This board failed at $9.2 \mathrm{krad}$ total integrated dose (TID). The Hyundai IC is used only on the C-THRU ROM single board computer.

An Altera EP32OPI PAL IC failed on the ROM-DOS single board computer, and all Maxim DG508A CMOS analog multiplexer IC's failed on the WinSystems AIO board. The ROM-DOS single board computer and the analog I/O board failed at $46 \mathrm{krad}$ TID-it is not clear which failed first.

It has been decided to use the ROM-DOS processor board in the cart electronic design. Since this processor survived $46 \mathrm{krad}$ TID during testing at Argonne, it should easily meet the $10 \mathrm{krad}$ TID over 5-year project design specification. This processor is also easier to program, and changes can be made to it easier than the C-THRU ROM processor. 


\section{APPENDIX C \\ BATTERY EVALUATION AND TEST RESULTS}

\section{INTRODUCTION}

Reliability of the WVNS transfer cart is of major importance. A cart failure during operation would prevent the transfer of waste canisters from the VC to the CPC storage area. Since storage area in the VC is limited to four canisters, operation of the vitrification process would be halted by the cart failure.

A major factor in cart reliability will be the battery system. The battery system must provide sufficient power to the cart to allow it to communicate with the facility computer and to power the cart drive motors for the duration of cart usage. Loss of power to the motors or to the cart control system would completely disable the cart. In order to insure that the battery will not be responsible for a cart failure due to lack of capacity, a battery assembly has been tested under loading conditions approximating those of the cart. Testing of the battery has provided data regarding the battery discharge profile and capacity.

\section{TEST OVERVIEW}

\subsection{BATTERY REQUIREMENTS}

Due to the voltage requirements of the cart telemetry system and drive motors, a $24 \mathrm{~V}$ battery will be used. The $24 \mathrm{~V}$ battery will consist of two $12 \mathrm{~V}, 25-40 \mathrm{~A}-\mathrm{h}$ batteries wired in series. Batteries wired in series operate at equal current. If the load is drawing $10 \mathrm{~A}$ from the $24 \mathrm{~V}$ battery assembly, each of the $12 \mathrm{~V}$ batteries "sees" a $10 \mathrm{~A}$ load current. This load current sharing allows for the testing of a single $12 \mathrm{~V}$ battery. Testing a single battery has the advantage of requiring a much smaller dynamic load and charging system. One consequence of using a single $12 \mathrm{~V}$ battery is that all test data reflects half the energy that would be delivered by a $24 \mathrm{~V}$ battery.

Four separate batteries have been used in testing. Two Gates Energy Products, 12 V, 25 A-h batteries (referred to as Gates A and B) and two Powersonic, 12 V, 40 A-h batteries (referred to as Powersonic $A$ and $B$ ). The Gates cells were tested since these batteries were used in cart preliminary design ${ }^{[1]}$. Recent ORNL test data ${ }^{[2]}$ has shown that Powersonic batteries perform better than the Gates cells in a radiation environment. For this reason, the Powersonic batteries have been tested as our primary candidate for use in the final cart design.

\subsection{DISCHARGE PROFILE}

The batteries were tested using a simulated cart discharge profile. This consisted of a controlled current discharge profile being applied to the battery over a period of several hours. The discharge profile was designed to approximate the battery loading during a cart operation cycle. Since the exact current 
requirements of the cart are not known at this time, tests at more than one idle current level were conducted. The initial tests used a cart idle current of $1.75 \mathrm{~A}$. A second abbreviated set of tests were conducted with an idle current of $3.0 \mathrm{~A}$. The discharge current profile was produced using a HewlettPackard model 6060A electronic load. The load was controlled by an IBM-PC through a general purpose interface bus (GPIB) interface. Battery current and voltage were be monitored during the discharge. Energy delivered by the battery has been computed from this data. Following the discharge, the battery was fully charged using a standard cyclic charge (battery charged at 2.45 volts per cell until charge current approaches zero amps). Battery charging voltage and current were monitored to establish the charging characteristics of the battery, total energy returned to the cell, and the time required to recharge the battery.

Figure C.1 shows the battery discharge current profile used in testing for a $1.75 \mathrm{~A}$ idle current. Figure C. 2 shows the battery discharge current profile used in testing for a $3.0 \mathrm{~A}$ idle current.

\subsection{TEST HARDWARE}

Figure C. 3 shows the hardware set up to be used in discharge testing. Table C. 1 contains a listing of the test hardware and pertinent data for each item in Figure C.3. Figure C.4 shows the hardware set up used for battery charge monitoring. Table C. 2 contains a listing of the test hardware and pertinent data for each item in Figure C.4. Calibration of all test equipment is documented in Table C.3.

Table C.1 Instrument Data for Fig. C.3.

\begin{tabular}{||l|l|l|}
\hline Item & Description & Purpose/Other Information \\
\hline IBM PC & IBM PC-AT computer & $\begin{array}{l}\text { Performs data logging, } \\
\text { display, and storage. }\end{array}$ \\
\hline GPIB interface & $\begin{array}{l}\text { National Instruments AT- } \\
\text { GPIB.2 }\end{array}$ & $\begin{array}{l}\text { Instrument control and data } \\
\text { acquisition through IEEE-488 } \\
\text { bus. Serial No. 18694. }\end{array}$ \\
\hline HP-6060A & $\begin{array}{l}\text { Hewlett-Packard Electronic } \\
\text { Load }\end{array}$ & $\begin{array}{l}\text { Provides computer controlled } \\
\text { current discharge profile. } \\
\text { Also digitizes voltage and } \\
\text { current for use by computer. } \\
\text { Serial number 3032A-01043. } \\
\text { Instrument is new and under } \\
\text { factory calibration. }\end{array}$ \\
\hline Battery under test & Sealed lead-acid battery & $\begin{array}{l}\text { Gates Energy Products 25 Ah } \\
\text { or Powersonic 40 Ah battery }\end{array}$ \\
\hline
\end{tabular}




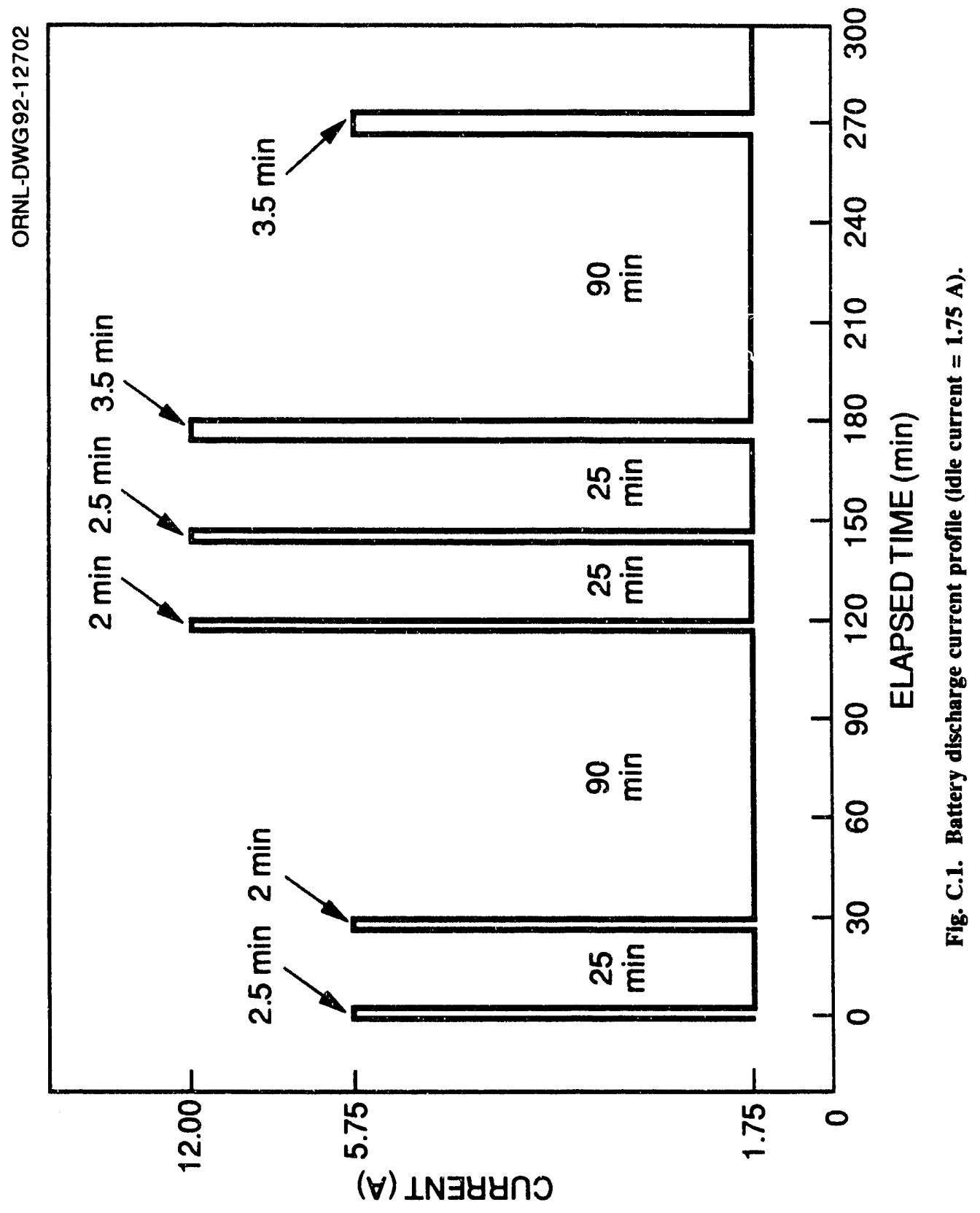




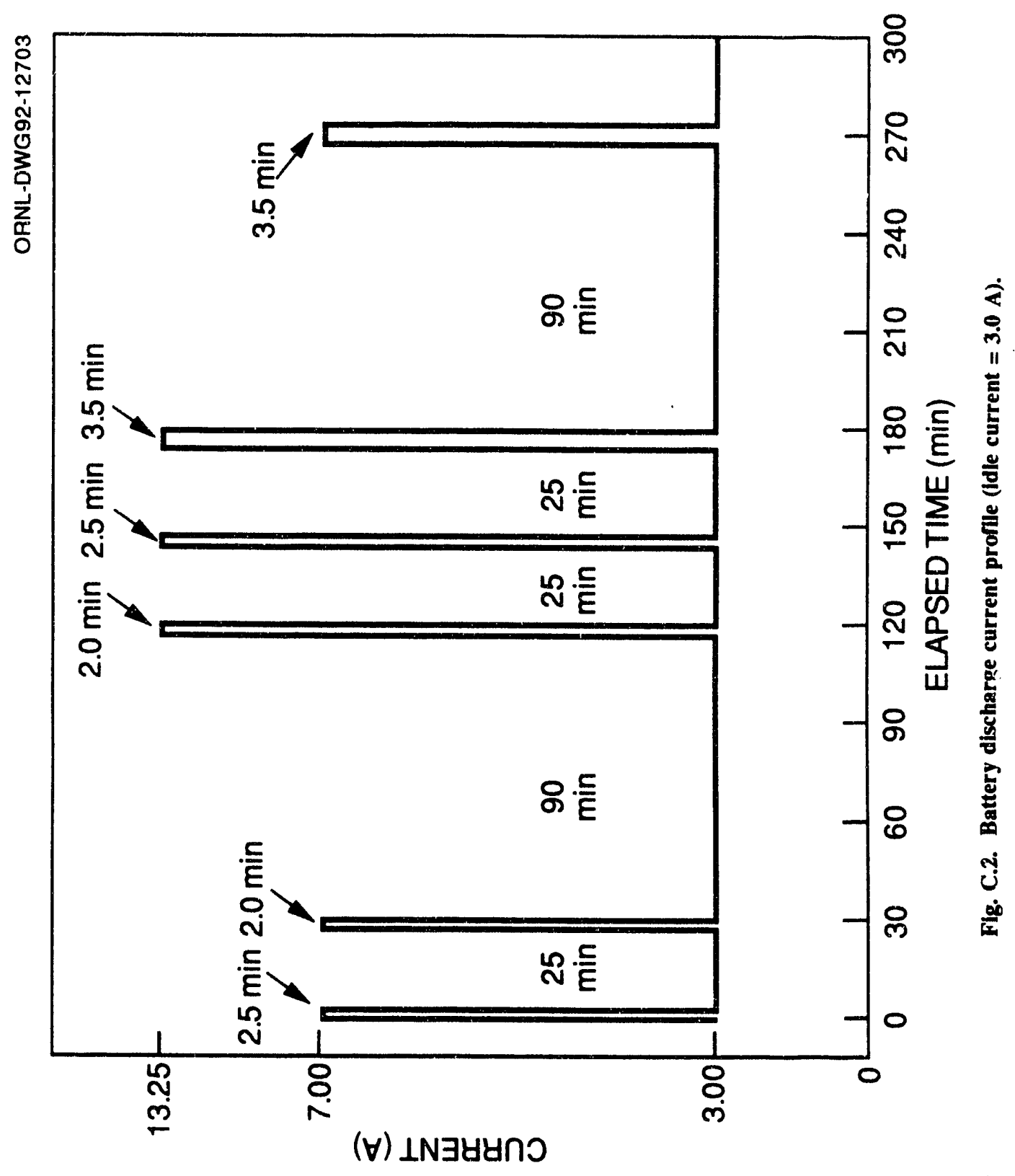




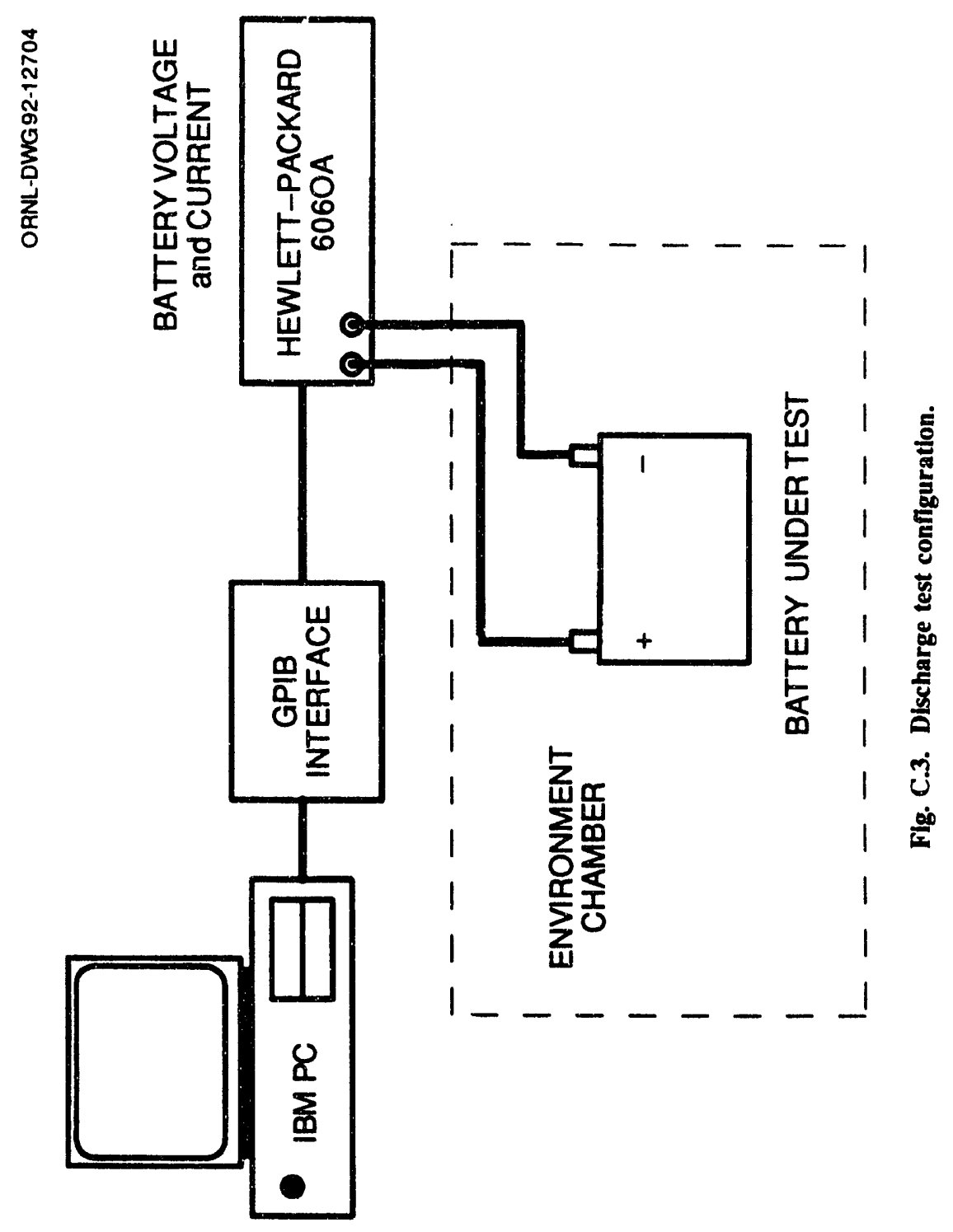


Table C.2 Instrument Data for Fig. C. 4.

\begin{tabular}{|l|l|l|}
\hline Item & Description & Purpose/Other Information \\
\hline IBM PC & IBM PC-AT computer & $\begin{array}{l}\text { Performs data logging, display, and } \\
\text { storage. }\end{array}$ \\
\hline GPIB interface & $\begin{array}{l}\text { National Instruments AT- } \\
\text { GPIB.2 }\end{array}$ & $\begin{array}{l}\text { Instrument control and data } \\
\text { acquisition through IEEE-488 bus. } \\
\text { Serial number 18694. }\end{array}$ \\
\hline Fluke 45, A & Fluke model 45 multimeter & $\begin{array}{l}\text { Provides digitized output of voltage } \\
\text { from current shunt. Serial number } \\
5115012 . \text { Unit is new and under } \\
\text { factory calibration. }\end{array}$ \\
\hline Fluke 45, B & Fluke model 45 multimeter & $\begin{array}{l}\text { Provides digitized output of voltage } \\
\text { from current shunt. Serial number } \\
5115032 . \text { Unit is new and under } \\
\text { factory calibration. }\end{array}$ \\
\hline Current shunt & Sensitive Research & $\begin{array}{l}\text { Current shunt. 50 mV at 30 A. } \\
\text { Serial number 934108. Resistance } \\
\text { to be verified by ORNL calibration } \\
\text { lab. }\end{array}$ \\
\hline 14.7 V, 10 A supply & Kepco model BOP 20-10M & $\begin{array}{l}\text { Current shunt. 50 mV at 30 A. } \\
\text { Serial number 934108. Resistance } \\
\text { to be verified by ORNL calibration } \\
\text { lab. }\end{array}$ \\
\hline Sealed lead-acid battery & $\begin{array}{l}\text { Gates Energy Products 25 A-h or } \\
\text { Powersonic 40 A-h battery. }\end{array}$ \\
\hline
\end{tabular}




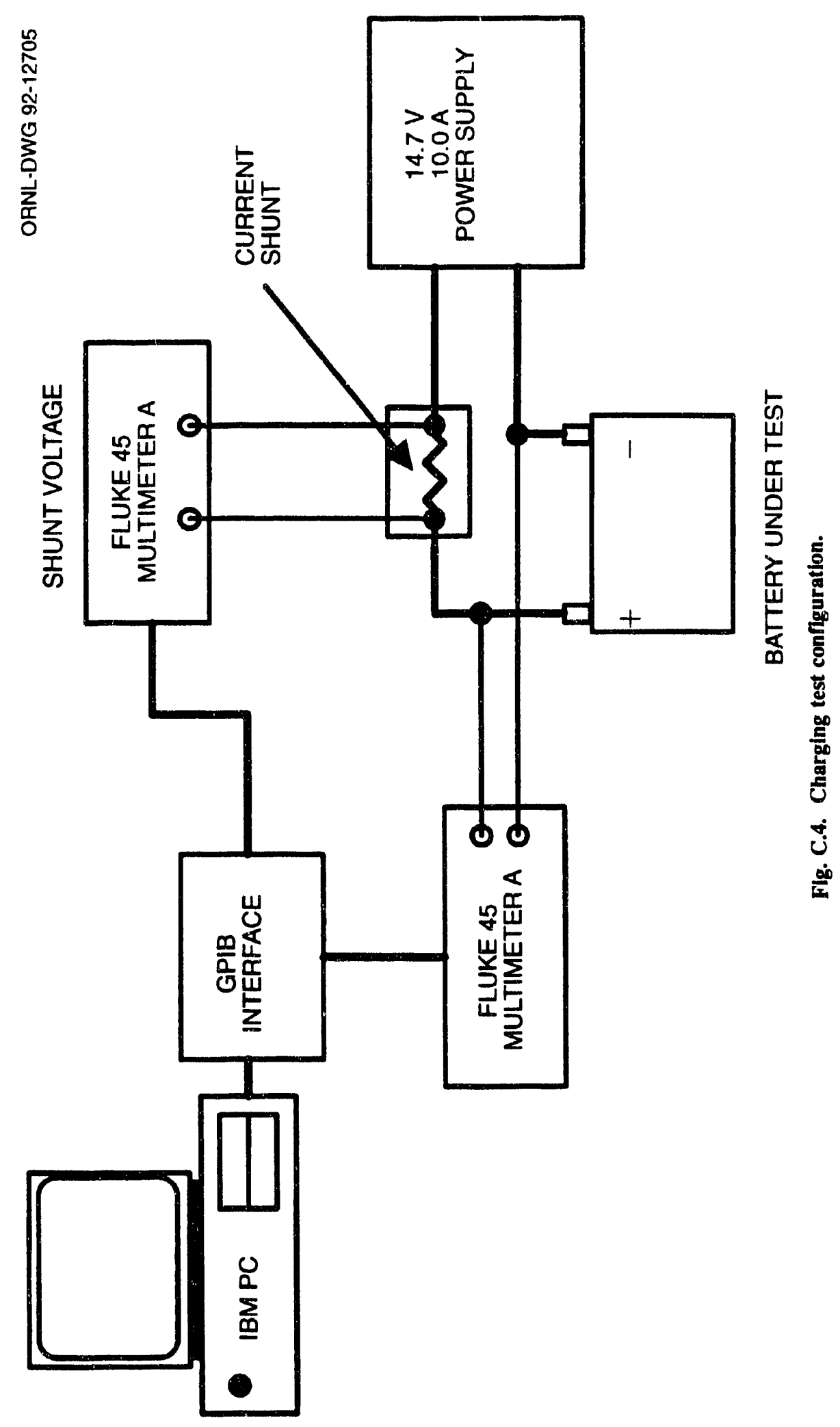


Table C.3 Instrument Calibration Data

\begin{tabular}{|l|l|}
\hline Instrument & Calibration \\
\hline $\begin{array}{l}\text { FLUKE model } 45 \text { multimeter. Serial number } \\
5115012 .\end{array}$ & Unit is new and under factory calibration. \\
\hline $\begin{array}{l}\text { FLUKE model } 45 \text { multimeter, Serial number } \\
5115032 .\end{array}$ & Unit is new and under factory calibration. \\
\hline $\begin{array}{l}\text { CURRENT SHUNT Sensitive Research } \\
\text { Current shunt. Serial number 934108. }\end{array}$ & $\begin{array}{l}\text { Calibrated by ORNL metrology group. } \\
\text { Certification Report ID IC2084. Resistance } \\
=0.00170 \text { 8. }\end{array}$ \\
\hline $\begin{array}{l}\text { Kepco model BOP 20-10M Power supply. } \\
\text { Serial number E122801. }\end{array}$ & $\begin{array}{l}\text { No calibration required (voltage and current } \\
\text { are externally monitored). }\end{array}$ \\
\hline $\begin{array}{l}\text { Hewlett-Packard Model 6060A. Serial number } \\
\text { 3032A-01043. }\end{array}$ & $\begin{array}{l}\text { Instrument is new and under factory } \\
\text { calibration. }\end{array}$ \\
\hline
\end{tabular}

\subsection{TEST ENVIRONMENT}

Tests were conducted at room temperature and at $20^{\circ} \mathrm{F}$. An environment chamber was used to chill the batteries to $20^{\circ} \mathrm{F}$ prior to, and during, the low temperature tests. The low temperature tests represent worst case conditions for battery performance during a discharge (high temperatures will impact battery life, but do not limit, and may enhance, the single cycle discharge performance of the battery). A limited number of test cycles were carried out at room temperature (nominally $70^{\circ} \mathrm{F}$ ) as a reference for "normal" battery performance.

\subsection{DATA LOGGING AND DOCUMENTATION}

A.ll test data and pertinent notes about tests were logged on an IBM-PC, with data files being stored on the PC's hard drive. Data was transferred to floppy disks on a daily basis. Initial data plots were be generated using Lotus 123 software in order to monitor progress of tests. Control of data logging was be done with a general purpose data logging program developed by $1 \& \mathrm{C}$ personnel. The code is written in Microsoft Quickbasic. Summary plots shown in section 3.5 of this document were generated with Microsoft Excel 3.0.

\section{TEST RESULTS}

\subsection{REVISED CART ENERGY REQUIREMENTS}

A revised energy requirement for the cart has been computed. Using data computed in the cart initial design ${ }^{[1]}$ combined with an updated cart current requirements data allows he new cart energy requirement to be computed. Tables C.4, C.5 and C.6 show a summary of these calculations. From these tables it can be seen that the cart battery must be sapable of providing $0.830 \mathrm{MJ}$ of energy under all anticipated 
cart operation scenarios for an idle current of $1.75 \mathrm{~A}$. This number will be used as a figure of merit in analyzing the $1.75 \mathrm{~A}$ battery test results. For the $3.0 \mathrm{~A}$ idle current, $1.31 \mathrm{MJ}$ of energy is required. This number will be used as a figure of merit in analyzing the $3.0 \mathrm{~A}$ battery test results. Note that these energy values are for a $24 \mathrm{~V}$ battery. All test data must be multiplied by a factor of 2 to correct for use of a single $12 \mathrm{~V}$ battery when comparing data with figures given in Tables C.4, C.5, and C.6.

Table C.4 Cart Idle Current Requirements

\begin{tabular}{|l|l|l|l|}
\hline System Element & Current (mA) & Voltage (V) & Power (W) \\
\hline System Controller & 100 & 5 & 0.5 \\
\hline Communications system & 2090 & 15 & 31.3 \\
\hline $\begin{array}{l}\text { Analog to Digital } \\
\text { Converter }\end{array}$ & 100 & 15 & 1.5 \\
\hline Multiplexer & 100 & 15 & 1.5 \\
\hline Motor Drive 1 & 50 & 24 & 1.2 \\
\hline Motor Drive 2 & 50 & 24 & 1.2 \\
\hline Motor Drive 3 & 50 & 24 & 1.2 \\
\hline Motor Drive 4 & 50 & 24 & 1.2 \\
\hline $\begin{array}{l}\text { Battery/Charger } \\
\text { Interface }\end{array}$ & 100 & 24 & 2.4 \\
& & & Total = 42.0 \\
\hline
\end{tabular}

Table C.5 Summary of Cart Energy Requirements (for $1.75 \mathrm{~A}$ idle current).

\begin{tabular}{||l|l|l|l|l|l|}
\hline Operation & $\begin{array}{l}\text { Time } \\
(\mathrm{min})\end{array}$ & $\begin{array}{l}\text { Drive } \\
\text { power } \\
(\mathrm{W})\end{array}$ & $\begin{array}{l}\text { Electrical power } \\
(\mathrm{W})\end{array}$ & $\begin{array}{l}\text { Total } \\
\text { power } \\
(\mathrm{W})\end{array}$ & $\begin{array}{l}\text { Energy } \\
(\mathrm{kJ})\end{array}$ \\
\hline \hline Idle time & 255 & 0 & 42.0 & 42.0 & 643 \\
\hline Moving, no load & 6.96 & 92.9 & 42.0 & 134.9 & 56.3 \\
\hline $\begin{array}{l}\text { Moving, full } \\
\text { load }\end{array}$ & 7.47 & 251 & 42.0 & 293 & 131 \\
\hline & & & & & 830 \\
\hline \multicolumn{1}{c}{ Totals } & 269.4 & & & & \\
\hline
\end{tabular}


Table C.6 Summary of Cart Energy Requirements (for 3.0 A idle current).

\begin{tabular}{||l|l|l|l|l|l|}
\hline Operation & $\begin{array}{l}\text { Time } \\
(\mathrm{min})\end{array}$ & $\begin{array}{l}\text { Drive } \\
\text { power } \\
(\mathrm{W})\end{array}$ & $\begin{array}{l}\text { Electrical power } \\
(\mathrm{W})\end{array}$ & $\begin{array}{l}\text { Total } \\
\text { power } \\
(\mathrm{W})\end{array}$ & $\begin{array}{l}\text { Energy } \\
(\mathrm{KJ})\end{array}$ \\
\hline \hline Idle time & 255 & 0 & 72.0 & 72.0 & 1100 \\
\hline Moving, no load & 6.96 & 92.9 & 72.0 & 165 & 68.9 \\
\hline $\begin{array}{l}\text { Moving, full } \\
\text { load }\end{array}$ & 7.47 & 251 & 72.0 & 323 & 145 \\
\hline & & & & & \\
\hline
\end{tabular}

\subsection{ROOM TEMPERATURE TESTS}

Typical room temperature data for the Gates Energy Products batteries are shown in Figures C.5 and C.6. Typical room temperature data for the Powersonic batteries are shown in Figures C.7 and C.8. For room temperature tests at low idle current $(1.75 \mathrm{~A})$, the Gates batteries provided an average energy of $0.776 \mathrm{MJ}$ (equivalent to $1.55 \mathrm{MJ}$ at $24 \mathrm{~V}$ ) during a discharge. The Powersonic batteries provided an average energy of $1.84 \mathrm{MJ}$ (crivilent to $3.68 \mathrm{MJ}$ at $24 \mathrm{~V}$ ) during a discharge for low idle current. For the Gates cell, that gives an energy delivered to energy required ratio of 1.87 for low idle current. For the Powersonic batteries the raiio is 4.43 for low idle current. For room temperature operation of the cart, the Gates and Powersonic batteries are acceptable for 1.75 idle current.

\subsection{LOW TEMPERATURE $\left(20^{\circ}\right.$ F) TESTS}

Typical $20^{\circ} \mathrm{F}$ temperature data for the Gates Energy Products batteries are shown in Figures C.9, C.10, C.11, and C. 12. Typical $20^{\circ} \mathrm{F}$ temperature data for the Powersonic batteries are shown in Figures C. 13 and C.14. For low temperature tests the Gates batteries provided an average energy of $0.798 \mathrm{MJ}$ (equivalent to $1.60 \mathrm{MJ}$ at $24 \mathrm{~V}$ ) during a discharge at low idle current $(1.75 \mathrm{~A}$ ) and $0.708 \mathrm{MJ}$ (equivalent to $1.42 \mathrm{MJ}$ at $24 \mathrm{~V}$ ) for high idle current $(3.0 \mathrm{~A})$. The Powersonic batteries provided an average energy of $1.46 \mathrm{MJ}$ (equivalent to $2.92 \mathrm{MJ}$ at $24 \mathrm{~V}$ ) during a discharge for low idle current and $1.09 \mathrm{MJ}$ (equivalent to $2.18 \mathrm{MJ}$ at $24 \mathrm{~V}$ ) for high idle current $(3.0 \mathrm{~A})$. For the Gates cell, that gives an energy delivered to energy required ratio of 1.93 for low idle current and 1.08 for hig'a idle current. For the Powersonic batteries the ratio is 3.52 for low idle current and 1.66 for high. idle current. For low temperature operation of the cart, Gates batteries are marginally acceptable for 1.75 and $3.0 \mathrm{~A}$ idle currents and the Powersonic batteries are acceptable at both 1.75 and $3.0 \mathrm{~A}$ idle currents.

\subsection{BATTERY CHARGING}

Typical battery recharging data for the Powersonic batteries are shown in Figures C.15 and C.16. The amount of energy returned to the cell is $2.75 \mathrm{MJ}$, which is consistent with the battery test discharges (additional energy is required to overcome battery losses during recharge). It takes approximately $7 \mathrm{~h}$ 


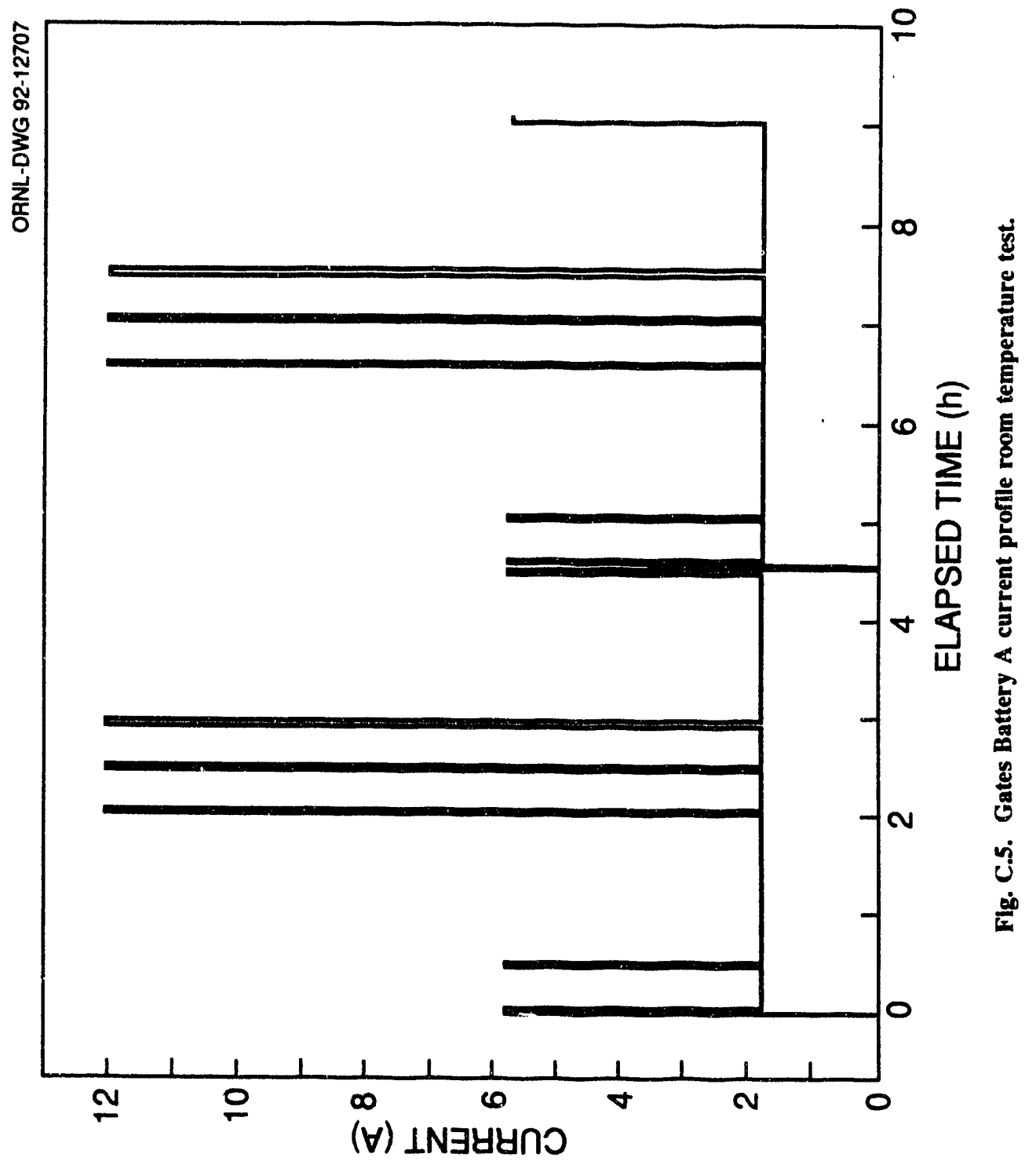




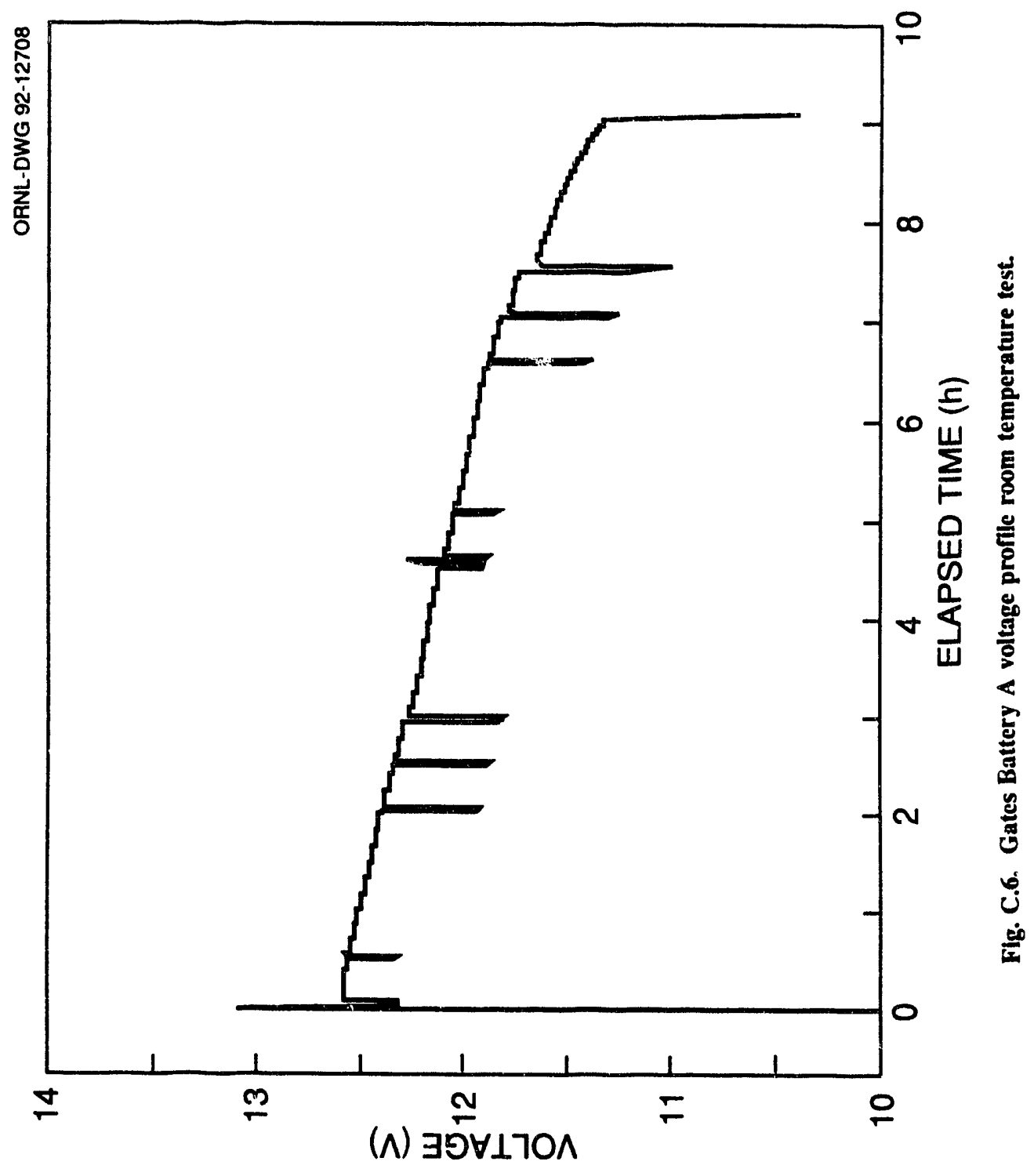




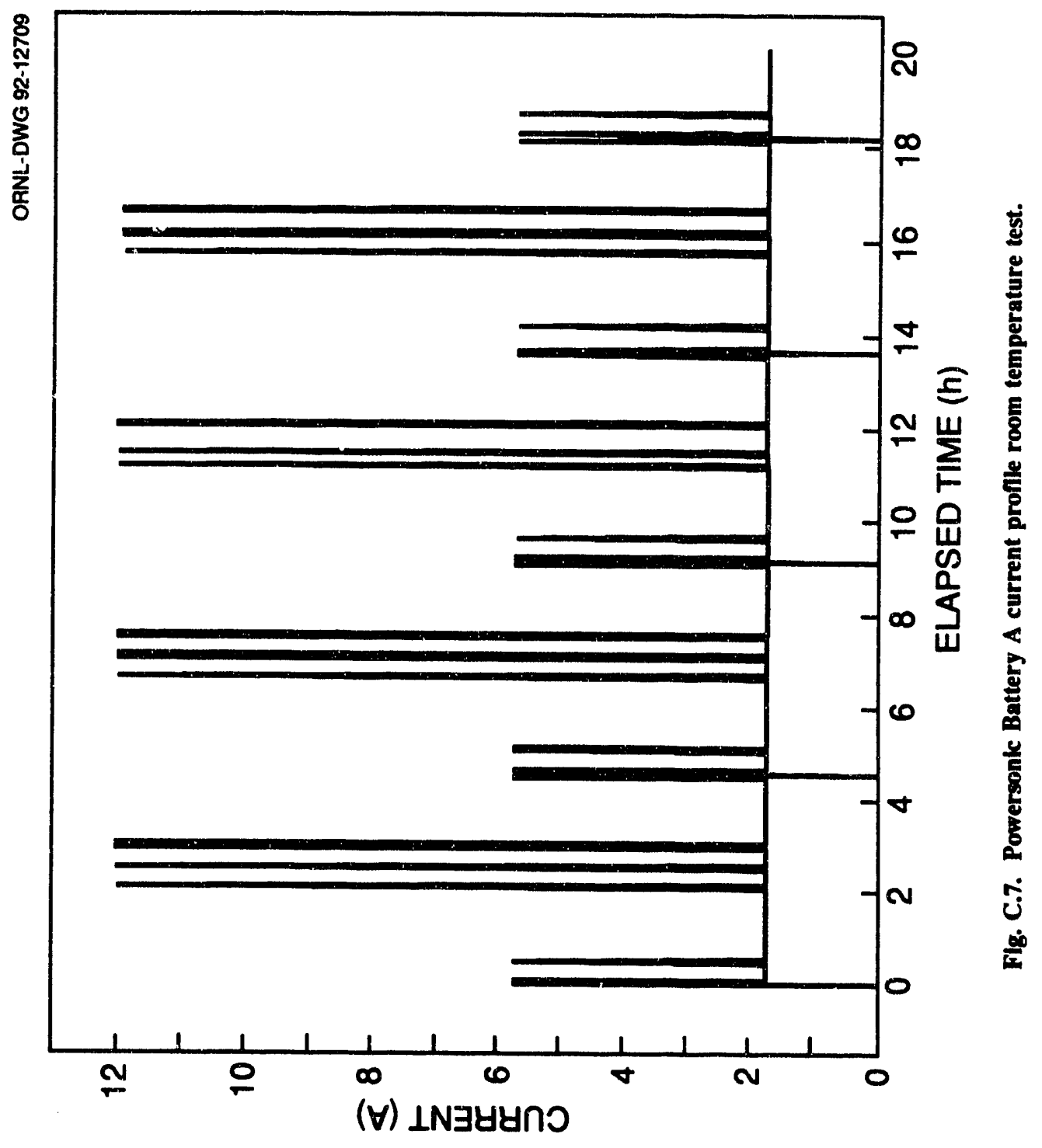




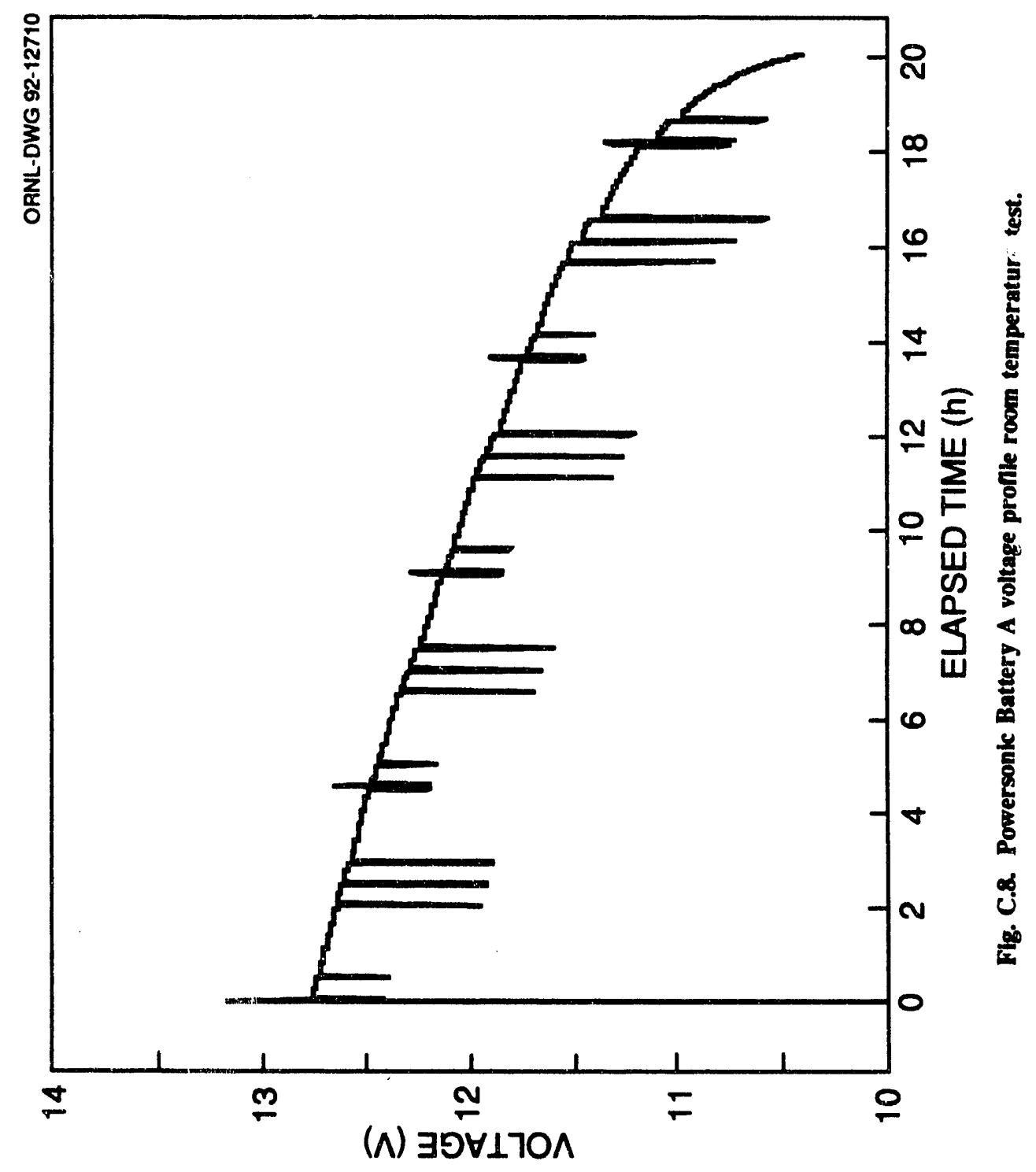




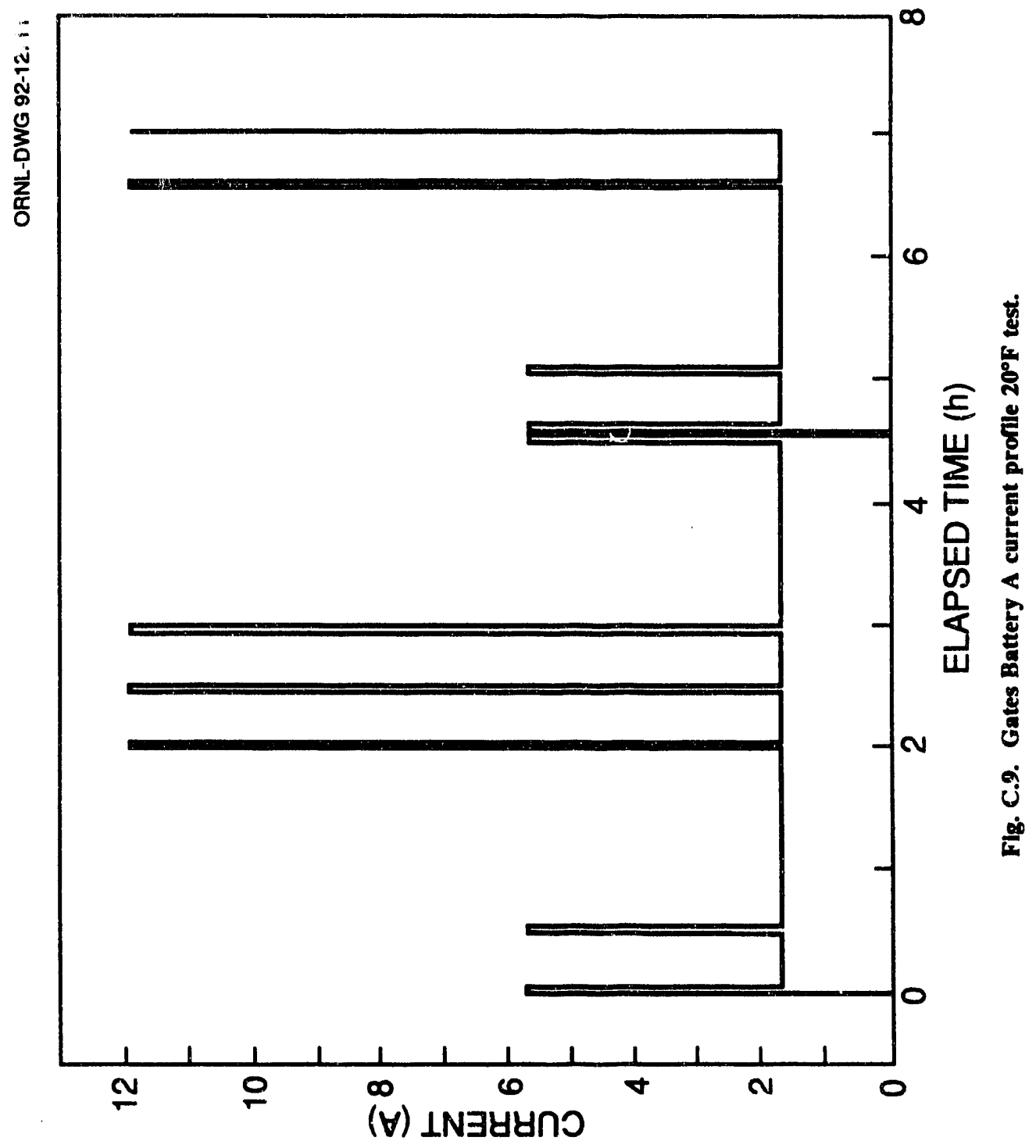




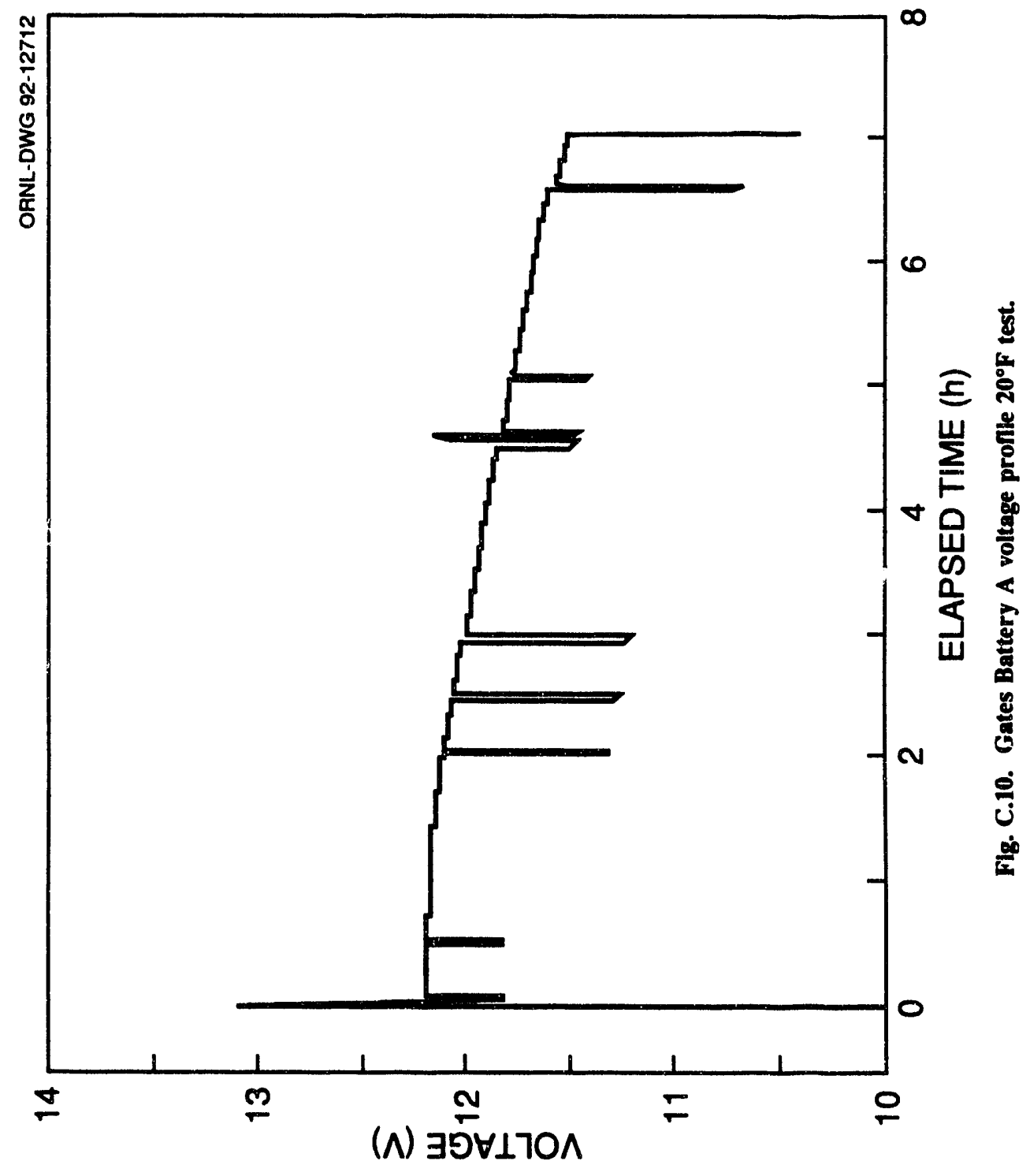




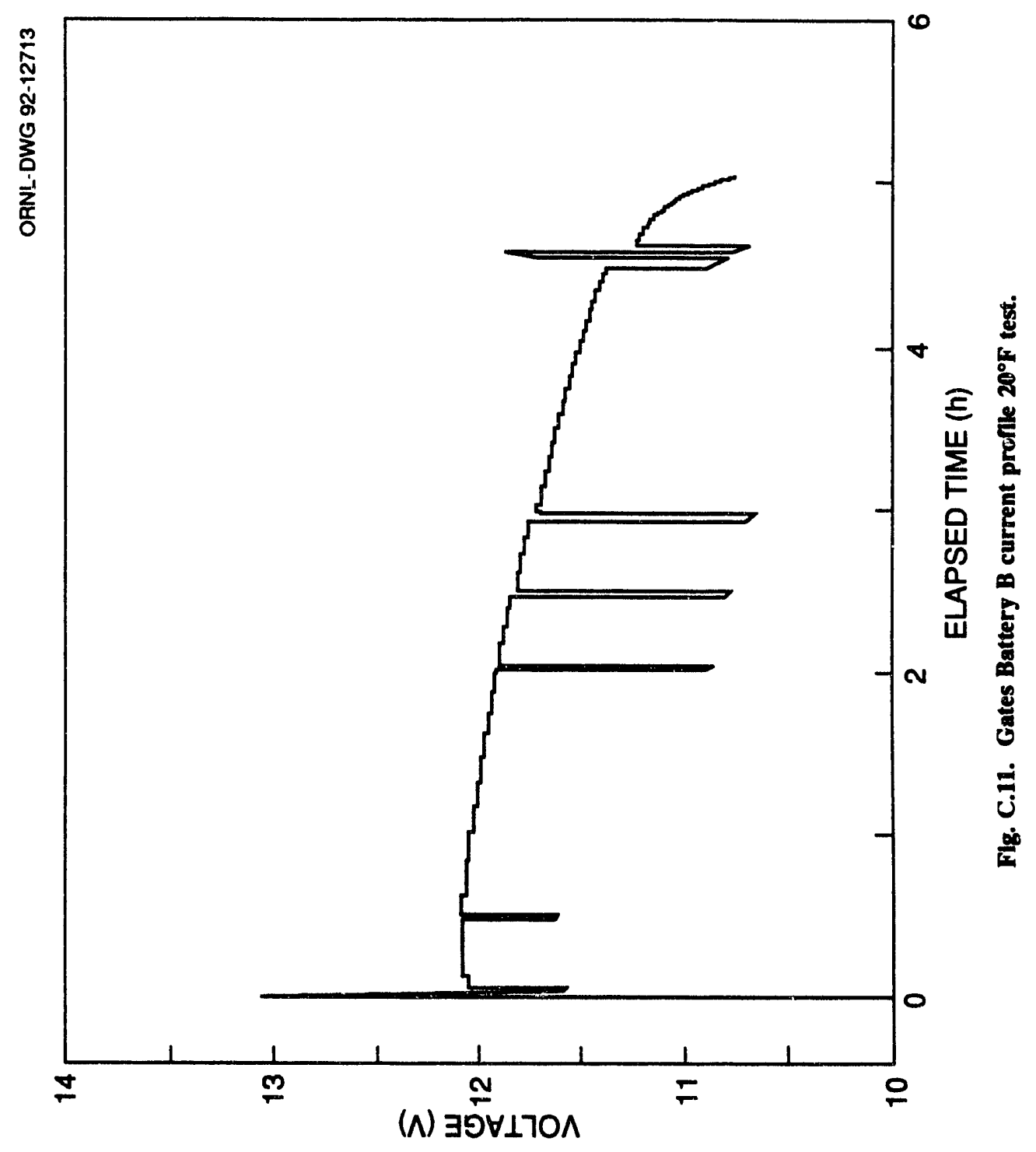




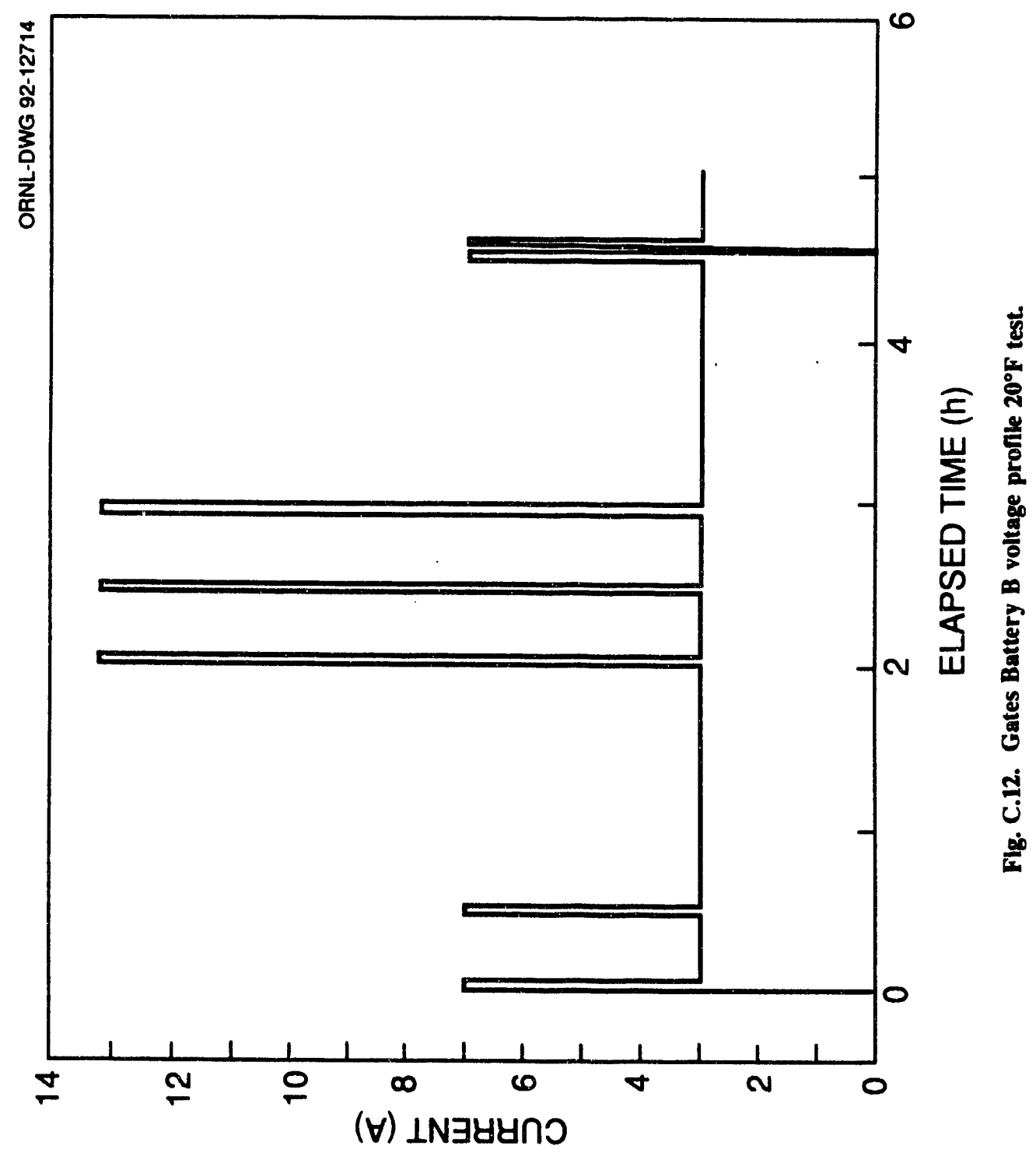




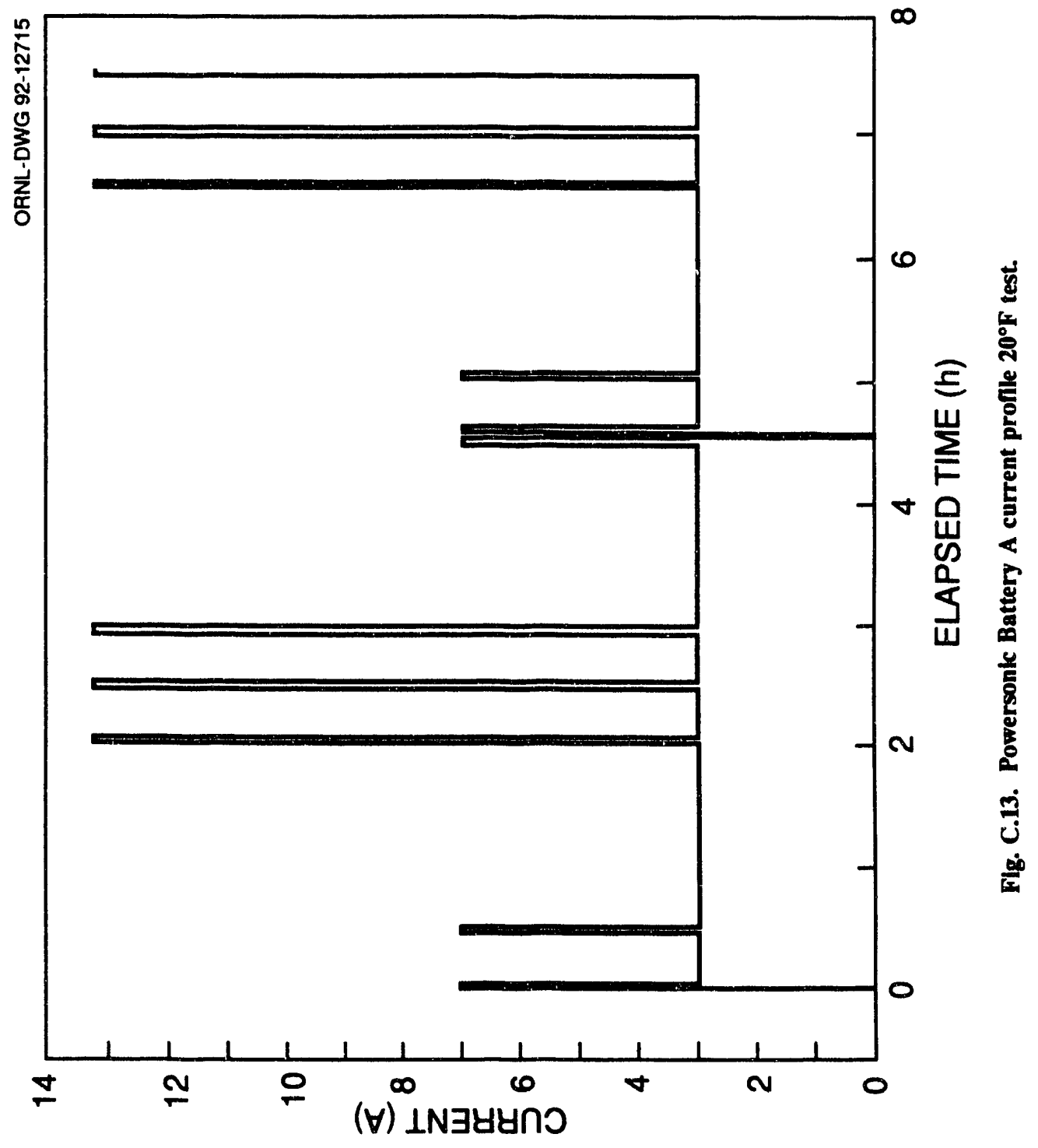




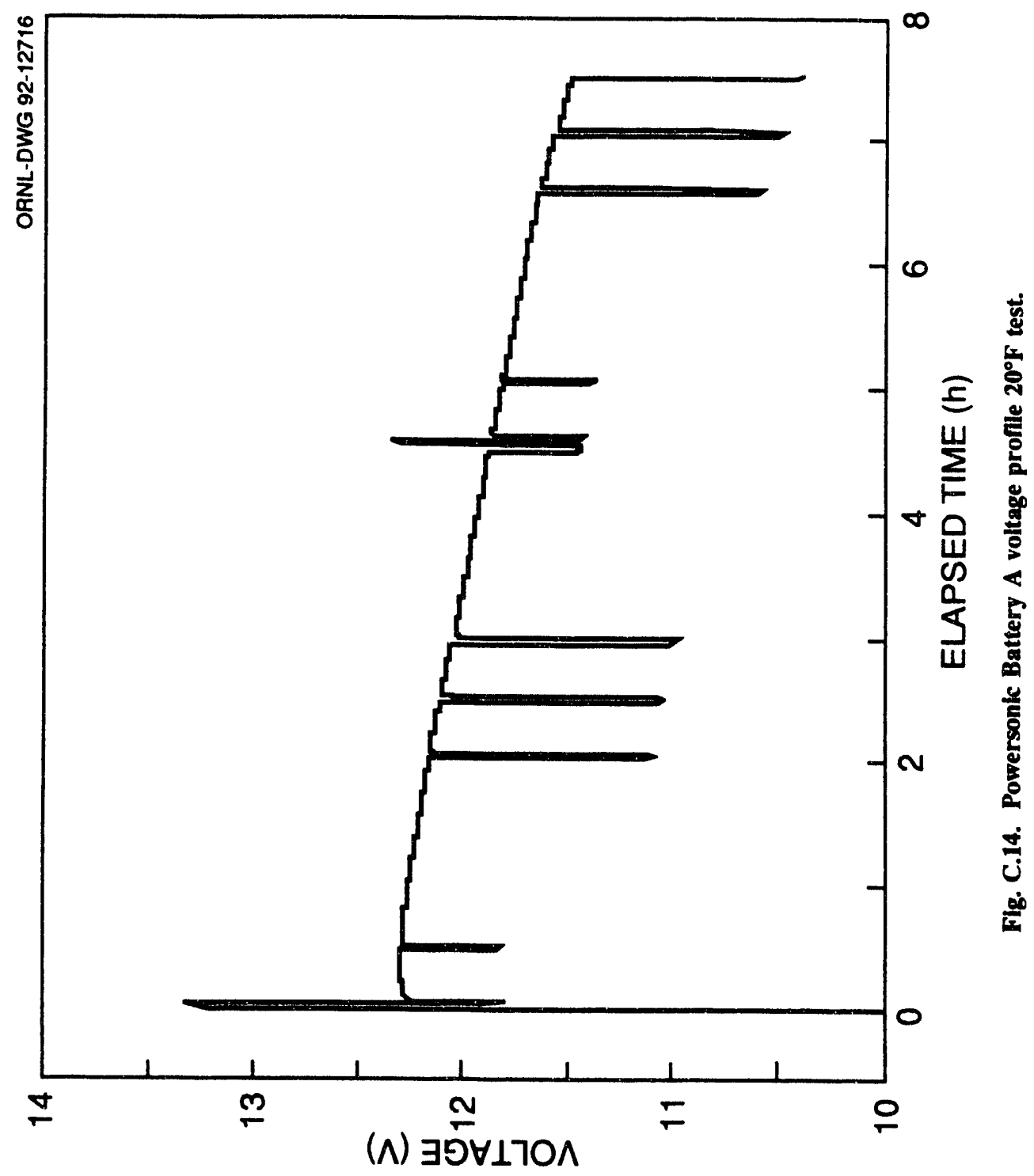




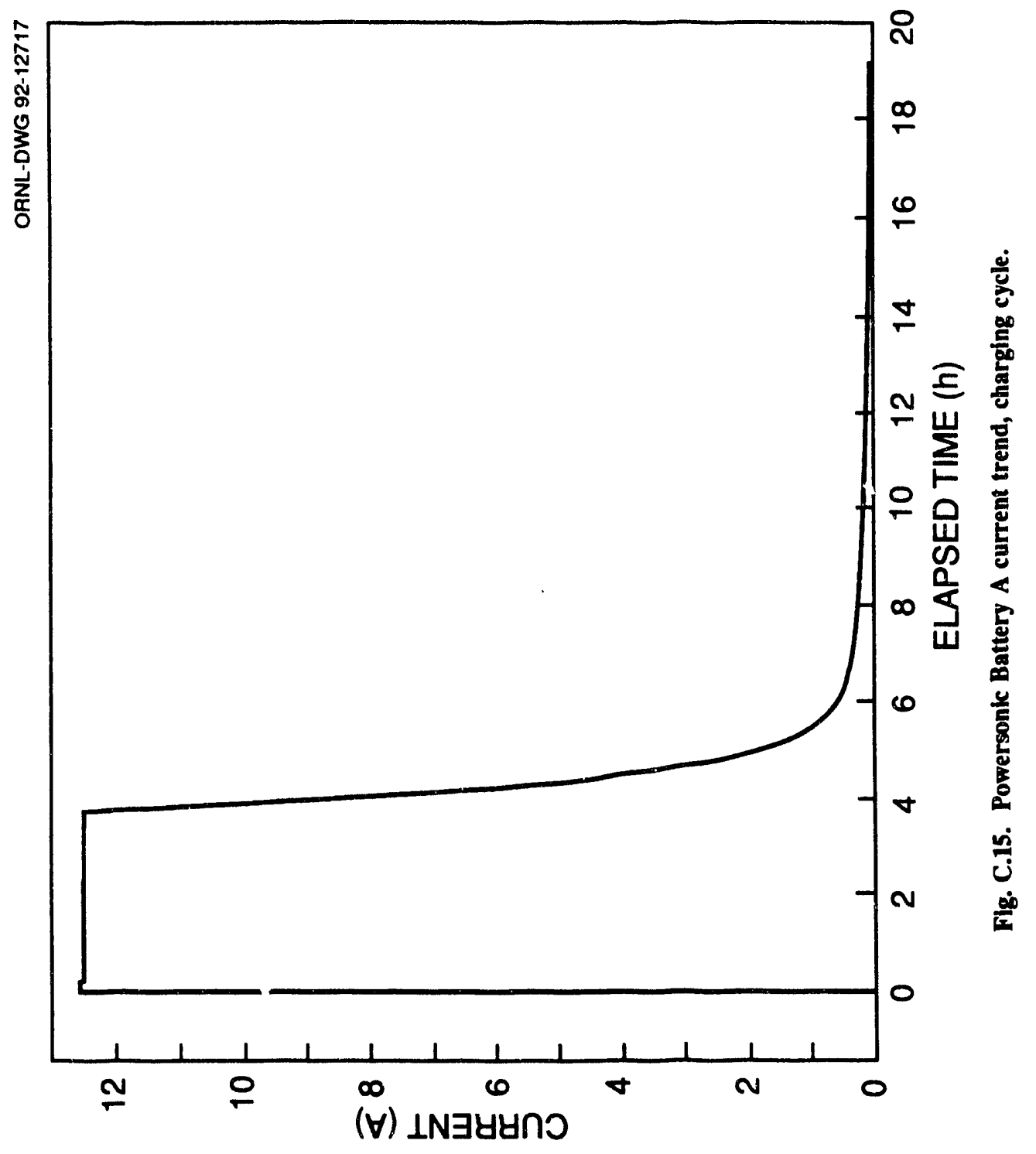




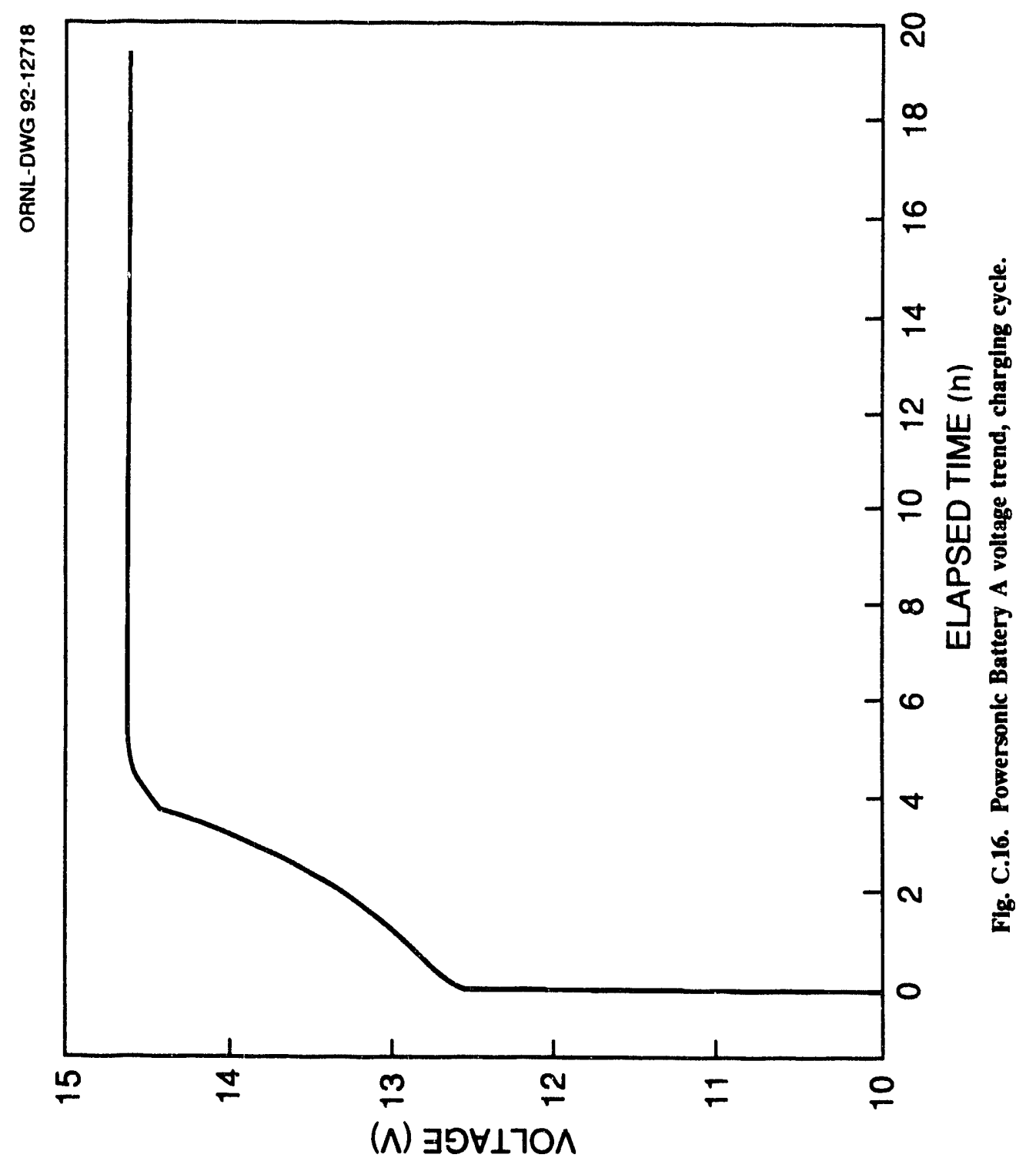


to provide a full charge after the Powersonic battery has delivered roughly $1.6 \mathrm{MJ}$ to a test load (equivalent to $3.2 \mathrm{MJ}$ at $24 \mathrm{~V}$ ). The trickle current which flows at the end of charge is approximately $60 \mathrm{~mA}$.

\subsection{SUMMARY}

The tests conducted for this report have shown that the Gates batteries tested are marginally acceptable for use in the cart design for a cart idle current of less than 3.0 A. The Powersonic batteries tested to be acceptable for use in the cart design at up to $3.0 \mathrm{~A}$ idle current. The Gatei batteries would probably provide for 1.5 cart cycles at end of life and $20^{\circ} \mathrm{F}$ for a $1.75 \mathrm{~A}$ idle current (assuming that the battery has $20 \%$ less capacity at end of life) and slightly less than 1 cycle at $3.0 \mathrm{~A}$ idle current. In comparing data sets there appears to be some inconsistency with the data for the Gates batteries. The computed total energies for the various discharges varied much more than the values found for the Powersonic batteries. Reviewing the voltage traces during discharge revealed a problem with our method of terminating a discharge. The high current voltage drop for the Gates battery was much larger near the end of battery life which caused the discharge to terminate (voltage less than $10.5 \mathrm{~V}$ ) earli, $r$ than necessary. For the Gates batteries a more accurate comparison of battery performance can be had by comparing data over the same time interval or by allowing termination of the discharge only during the idle current periods.

The Powersonic batteries should have sufficient capacity, even at $20^{\circ} \mathrm{F}$ and end of life to operate the cart for 2.5 cycles for a $1.75 \mathrm{~A}$ idle current (again, assuming that the battery has $20 \%$ less capacity at end of life) and slightly more than 1 cycle at $3.0 \mathrm{~A}$ idle current and end of life.

Figure C.17 shows a typical discharge profile energy and current versus elapsed time for a Powersonic battery at $1.75 \mathrm{~A}$ idle current. Periods of high current operation only account for roughly $13 \%$ of the total time the cart can operate. Battery life for the cart remaining idle during the full operation time would be about $18 \mathrm{~h}$ (this value is computed by extending the idle current line slope from the origin of the plot in Figure C. 17 out to the energy level where battery operation terminated in the test) for the Powersonic battery at a $1.75 \mathrm{~A}$ idle current.

Figure C.18 shows the energy returned to the battery and current during charging for a Powersonic battery. From this graph it can be seen that $94 \%$ of the energy accepted by the battery is transferred in the first $5 \mathrm{~h}$ of charging (for a charger limited to roughly $10 \mathrm{~A}$ ). Only $6 \%$ of the $2.75 \mathrm{MJ}$ accepted by the battery is transferred between 5 and $13 \mathrm{~h}$ of elapsed time. For a cart operated over a single cycle, it would be possible to recharge the battery in much less than $5 \mathrm{~h}$ since the amount of energy removed from the battery would be a factor of two or more smaller than for the data shown. 


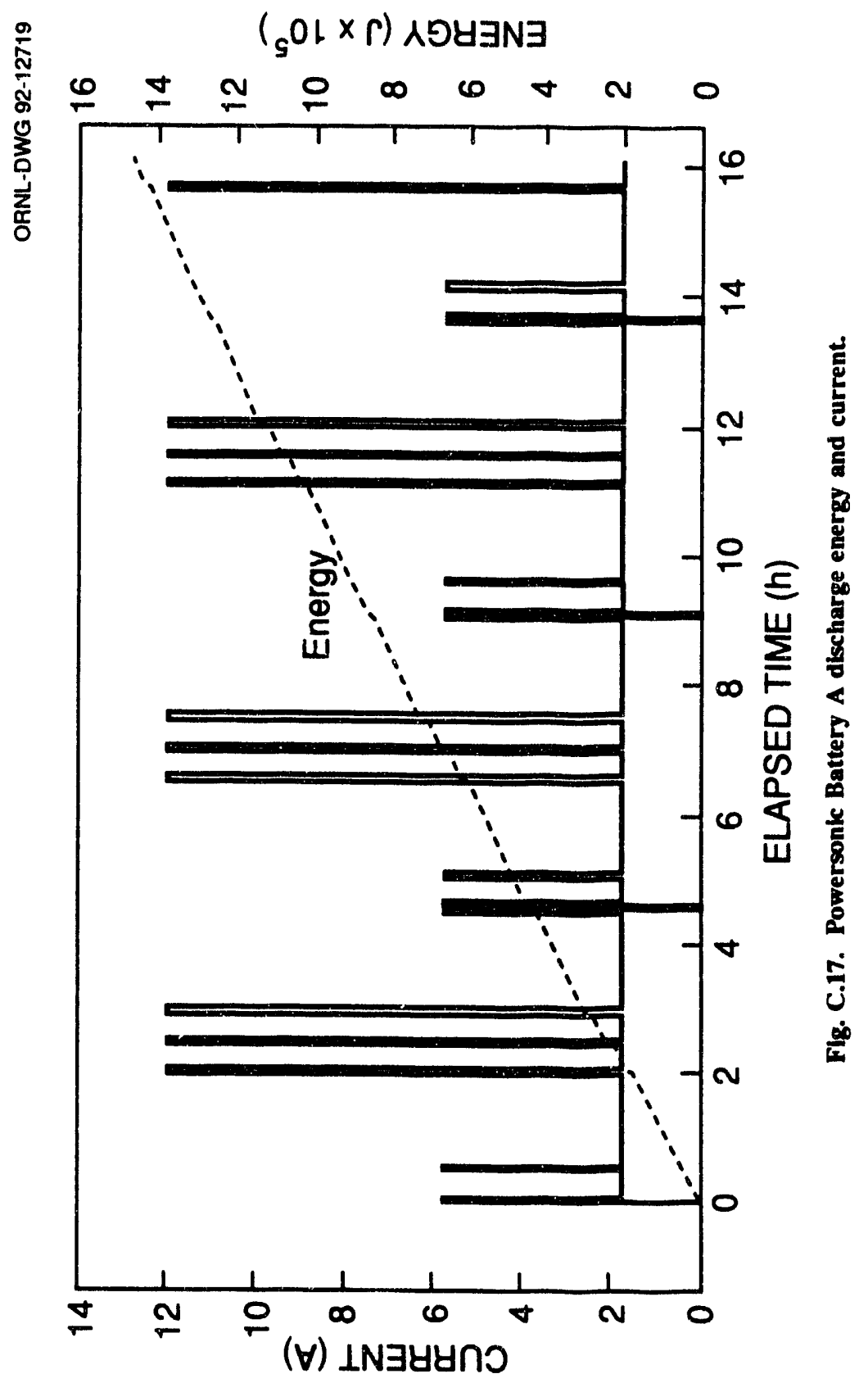




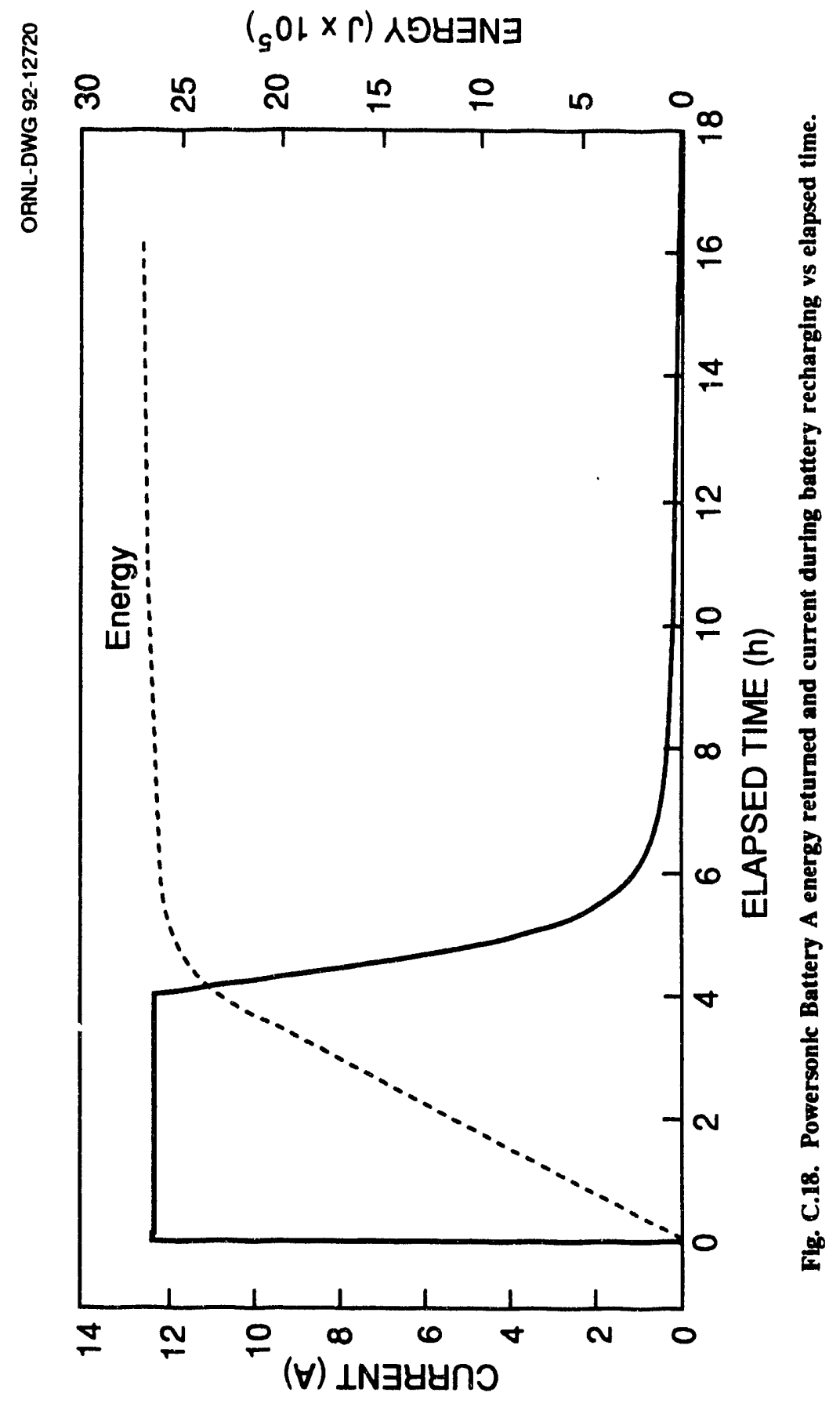




\section{REFERENCES}

1. "Preliminary Control System Design Description," West Valley Demonstration Project, Vitrification Facility Transfer Cart, December 21, 1990.

2. Weil, Brad. ORNL. Verbal communication, May, 1991. 


\section{APPENDIX D \\ PROTOTYPE HARDWARE TESTING}

\section{INTRODUCTION}

The purpose of this appendix is to document testing of the prototype system hardware and software for the subject control system which occurred in early 1992 . The intent of the testing was to perform a system-wide test of the prototype hardware. Testing of each component had already been done before the system test began. The overall functionality of the system was under test. For example, in the motor test, a button was pushed on the control pendant and motor operation was witnessed. Many parts of the system had to be operating for these functions to occur.

\section{COMPONENTS}

Prototype hardware that was tested is listed in the following table. It was arranged as shown Figure D.1. The whole system including if system was tested, but at first the communications protocol was tested by a hard-wired link between the facility side and cart side equipment as shown by the dotted line in the figure. Software, which is part of the design and which was also tested in this procedure, was running in the programmable logic controller, the engineer's console computer, the facility-side STDbased controller, and the cart-side STD-based controller.

Table D.1. Prototype Hardware for Functional Test

\begin{tabular}{|l|l|}
\hline Component & $\begin{array}{l}\text { Make and Model or } \\
\text { Reference Drawing }\end{array}$ \\
\hline Operator's Control Pendant & Q-6340-120 \\
\hline Programmable Logic Controller & \\
\hline Processor & Allen-Bradley PLC 5/15 \\
Digital Inputs Board & Allen-Bradley 1771-IBD \\
Digital Output Boards (2) & Allen-Bradley 1771-OBD \\
Simulation Modules (2) & Allen-Bradley 1771-SIM \\
\hline Engineer's Console Computer & Gateway 486/33C \\
Interface Card & Sutherland-Shultz 5136 SD \\
\hline Eacility-side STD-based Controller & \\
Processor & Prolog 7870-01 \\
Memory & Prolog 7715A-03 \\
Digital Interface & Prolog 7508 \\
Allen-Bradley Interface & Prolog 7514 \\
Encoder/Decoder & Q-6340-187 \\
\hline
\end{tabular}




\begin{tabular}{|c|c|}
\hline Component & $\begin{array}{l}\text { Make and Model or } \\
\text { Reference Drawing }\end{array}$ \\
\hline Maintenance/Programming Computer & IBM AT \\
\hline $\begin{array}{l}\text { Facility-side If cage } \\
\text { Facility Antenna Switch } \\
\text { Facility Antenna Switch } \\
\text { Facility Transmitter } \\
\text { Facility/Cart Receiver }\end{array}$ & $\begin{array}{l}Q-6340-181 A \\
Q-6340-181 B \\
Q-6340-171 \\
Q-6340-261\end{array}$ \\
\hline $\begin{array}{l}\text { Digital Interface Board } \\
\text { Digital Output Module } \\
\text { Digital Input Module }\end{array}$ & $\begin{array}{l}\text { Opto-22 PB16H } \\
\text { Opto-22 ODC5 } \\
\text { Opto-22 IDC5B }\end{array}$ \\
\hline Facility Transmit/Receive Circuit & Q-6340-175 \\
\hline Battery Charger & Exide ERBC 24/30 \\
\hline Battery Charger Polarity Manager & $Q-6340-135$ \\
\hline Monopole Antennas (3) & $\begin{array}{l}\text { N/A (steel rods cut to length with } \\
\text { groundplanes) }\end{array}$ \\
\hline $\begin{array}{l}\text { Cart-side if Cage } \\
\text { Facility/Cart Receiver } \\
\text { Cart Transmitter } \\
\text { Cart Antenna Controller }\end{array}$ & $\begin{array}{l}Q-6340-261 \\
Q-6340-271 \\
Q-6340-266\end{array}$ \\
\hline $\begin{array}{l}\text { Cart-side STD-based controller } \\
\text { Processor } \\
\text { Digital } 1 / 0 \\
\text { Analog I/O } \\
\text { Interface } \\
\text { Cart Encoder/Decoder }\end{array}$ & $\begin{array}{l}\text { Winsystems SBC40A } \\
\text { Winsystems SBX-PIO } \\
\text { Winsystems LPM-AIO-DC } \\
\text { Q-6340-220,225,230 } \\
\text { Q-6340-291 }\end{array}$ \\
\hline Maintenance/Programming Terminal & C. Itoh CIT 101 \\
\hline Batteries (2) & Powersonic PS-12400 \\
\hline Motor & Pacific Scientific BA3618-7009-9-48C \\
\hline Motor Controller & Q-6340-235 \\
\hline Limit Switch & Microswitch 1LS1 \\
\hline Thermocouple & Type E thermocouple wire \\
\hline
\end{tabular}




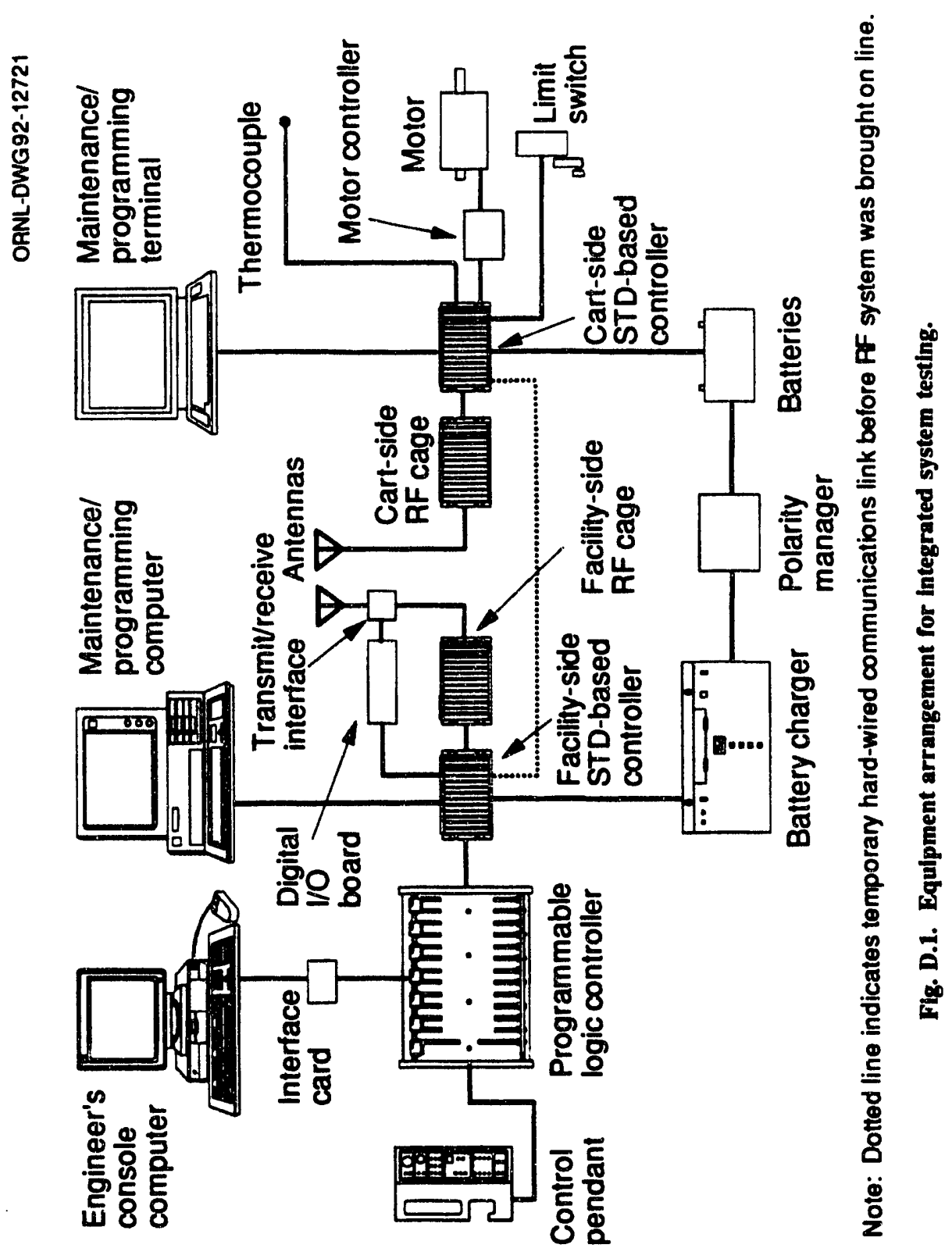




\section{FUNCTIONAL TESTS}

Functional test results reported here bave been arranged into three categories: functions initiated from the control pendant, functions initiated from the engineer's console, and functions initiated from the cart system. The following information is given for each functional test: functional test title, test description, expected result, witnessed result, considerations, test performer, and date of test. Considerations are given to point out subtleties of each function that may not be apparent on the surface or to point out efforts that should be undertaken later.

Functional Test:

Emergency Stop

Test Description:

An emergency stop switch is provided on the control pendant. Unlike all other functions on the pendant (except for the LED test), the emergency stop function does not require the station to be enabled before it is active.

Expected Result:

The emergency stop should prevent cart drive, motor resets, auxiliary outlet operation, and stop all communications with the cart system. It should also stop battery charging if it is in effect.

Witnessed Result:

While the battery charger was active (and cart electronics inactive), the emergency stop button was pushed. Battery charging ceased and the cart electronics reactivated. The emergency stop LED of the control pendant lit, and further requests for other operations resulted in the LED flashing.

While the cart motor drive was active, the emergency stop button was pushed. The cart motor stopped rotating and further communications with the cart ceased (telemetry data was no longer active).

Consideration:

Because the emergency stop function eliminates communications with the cart, requests for cart data cannot be sent. Therefore, during an emergency stop condition, cart status is no longer known.

Because there are only two operating modes of the cart-battery charging and cart operation-there is no way to "kill" the cart. When emergency stopped, the cart will be in an idle mode, not completely dead.

Tested by: F. R. Ruppel

Date: $\quad 2-3-92$ 
Functional Test:

Control Station Select

Test Description:

Only one control station will be enabled at one time. The selection is on a first-come, firstserved basis by the use of a keyswitch on the control pendant.

\section{Expected Result:}

With no other station enabled, the operator should be able to enable his station by inserting a key and rotating it to the on position. The LED for this station should light. If another user is already enabled, it should not be possible to enable another station.

Witnessed Result:

With no other station was selected, the station select keyswitch was turned to the on position. The LED for this station lit and functions were enabled from the operator's pendant. The station select keyswitch was turned off, and the input which indicates when the other control pendant is selected was enabled. Attempts to select this control station were ineffective. The LED for the other station flashed, indicating that it was selected.

\section{Consideration:}

Since the station select is key operated, distribution of keys should be administered only to operators qualified to operate the cart and battery charger. It should not be allowed for an operator to walk away from the control pendant without taking the key with him.

Tested by: F. R. Ruppel

Date: $\quad 1-9-92$ 
Functional Test:

LED Test

Test Description:

Each control pendant will have a feature of testing its own LEDs and audible alarms to confirm their operation.

Expected Result:

Each LED of the control pendant should begin flashing, regardless of previous state, when the keyswitch is rotated to the LED test position. The audible alarm should sound also.

Witnessed Result:

The keyswitch was turned to the LED test position. All LEDs flashed and the audible alarm sounded. When the key was returned to the off position, the LEDs ceased flashing, returned to their previous state, and the audible alarm was silent.

Consideration:

To perform the LED test, the keyswitch must be rotated to the LED test position. If the station is currently enabled, it will be disabled when going to the LED test mode. If a second operator at the other station is waiting to enable his station, he may enable it while the first operator goes to LED test mode, thus locking out the first operator.

Tested by: F. R. Ruppel

Date: $\quad 1-9-92$ 
Functional Test:

Door LEDs

Test Description:

LEDs are provided for door open, door closed, and door energized for all three doors in the cart path.

\section{Expected Result:}

The LED should reflect the input for all door states.

Witnessed Result:

Inputs were simulated for door open, door closed, and door energized for doors 63M-001, 63M008 , and 3M-3. Each LED reflected its appropriate status.

\section{Consideration:}

When a door is energized, it prevents any cart operation. Fail safe circuitry of the door energized inputs should be addressed to assure that a failed door energized status will also prevent cart operation. Likewise, when the cart is driving within a certain distance of an unopen door, the operator will be notified. Fail safe circuitry of the door open limit switches should be addressed also.

Tested by: F. R. Ruppel

Date: $\quad 1-10-92$ 
Functional Test:

Motor Drive

Test Description:

Using the control pendant, it should be possible to drive the motor clockwise and counterclockwise by using the drive north/drive south button. The appropriate motor voltage and current should appear on engineer's console display.

Expected Result:

Motor should rotate when north drive button pushed on control pendant and rotate in opposite direction when south drive button pushed. Engineer's console should read motor voltage and current.

Witness Result:

All motors indicated $0.0 \mathrm{~A}$ and $-1.0 \mathrm{~V}$ at the engineer's console display when the motor was not running. (The $-1.0 \mathrm{~V}$ is an offset error that was later corrected.)

Motor $\# 1$ indicated $+13.1 \mathrm{~V}$ and $0.4 \mathrm{~A}$ when the south drive button was pushed and $-15.0 \mathrm{~V}$ and $0.4 \mathrm{~A}$ when the north drive button was pushed.

Motor $\# 2$ indicated $+14.0 \mathrm{~V}$ and $0.5 \mathrm{~A}$ when the south drive button was pushed and $-16.1 \mathrm{~V}$ and $0.5 \mathrm{~A}$ when the north drive button was pushed.

Motor $\# 3$ indicated $+14.0 \mathrm{~V}$ and $0.6 \mathrm{~A}$ when the south drive button was pushed and $-15.9 \mathrm{~V}$ and $0.6 \mathrm{~A}$ when the north drive button was pushed.

Motor \#4 indicated $+13.6 \mathrm{~V}$ and $0.6 \mathrm{~A}$ when the south drive button was pushed and $-15.3 \mathrm{~V}$ and $0.6 \mathrm{~A}$ when the north drive button was pushed.

Consideration:

As set up in hardware and software, motor voltages are reported either positive or negative depending on rotation direction. For historical logging, this will indicate which direction the motor was rotating at any given time. However, if historical values of average absolute voltage are desired, or any other absolute measurement, the data will have to be modified.

Tested by: $\quad$ F. R. Ruppel and A. V. Blalock

Date: $\quad 1-7-92$ 
Functional Test:

Battery charging

Test Description:

Battery charging is one of the more complex operations performed by the operator.

Expected Result:

From the control pendant, the operator should be able to start and stop charging after the cart-atshoes input is enabled and if the cart is not driving. The emergency stop switch should stop charging. Charging variables should be displayed on the engineer's console. Cart electronics should disable when charging commences. Polarity to the cart batteries should be switchable by the cart orientation switch.

Witnessed Result:

Charging was initiated by pressing the charge start button on the control pendant. The battery charger started and data was read at the engineer's console as follows: external voltage - 24.4 $\mathrm{V}$, internal voltage $-24.7 \mathrm{~V}$, current $-1.9 \mathrm{~A}$, external temperature $-87.1^{\circ} \mathrm{F}$, internal temperature $-86.7^{\circ} \mathrm{F}$. The battery charging sequence was stopped by pressing the control pendant emergency stop button. The charger stopped and the cart system started. Charging was reinitiated and the charger was stopped again, this time by pressing the stop charging button on the control pendant. The next day, a full charge was given to the batteries. When charge current dropped below $0.08 \mathrm{~A}$, the charging complete light on the control pendant lit up

When the cart orientation switch was changed, polarity to the cart batteries reversed.

Consideration:

The cart orientation switch should be under strict administrative control since it also controls charging polarity. Attempting to charge the batteries with polarity reversed could harm equipment.

Tested by: $\quad$ F. R. Ruppel

Date: $\quad 2-3$ and $2-4-92$ 
Functional Test:

Control Pendant Undercurrent Alarms

\section{Test Description:}

This procedure tests the motor undercurrent alarm at the control pendant. This alarm is designed to indicate to the operator when a wheel is free-wheeling. Use the control pendant to drive the motor; ensure that the motor current is monitored by the cart electronics and transmitted to the facility-side control system.

Expected Result:

During a low motor current condition, motor LEDs should flash after a $2 \mathrm{~s}$ time delay.

Witnessed Result:

Motor $\# 1$ was started with no load (current $=0.4 \mathrm{~A}$ ). After $2 \mathrm{~s}$, motor \#1 LED flashed at the control pendant. When the motor was loaded, (current $=1.2 \mathrm{~A}$ ) the LED stopped flashing.

Motor $\# 2$ was started with no load (current $=0.5 \mathrm{~A}$ ). After $2 \mathrm{~s}$, motor \#1 LED flashed at the control pendant. When the motor was loaded, (current $=1.3 \mathrm{~A}$ ) the LED stopped flashing.

Motor \#3 was started with no load (current $=0.7 \mathrm{~A}$ ). After $2 \mathrm{~s}$, motor \#1 LED flashed at the control pendant. When the motor was loaded, (current $=1.3 \mathrm{~A}$ ) the LED stopped flashing.

Motor $\# 4$ was started with no load (current $=0.6 \mathrm{~A}$ ). After $2 \mathrm{~s}$, motor $\# 1$ LED flashed at the control pendant. When the motor was loaded, (current $=1.2 \mathrm{~A}$ ) the LED stopped flashing.

Consideration:

The low current threshold is set at $1 \mathrm{~A}$. During actual cart testing, this value may need to be adjusted in the PLC ladder logic to correctly reflect a free-wheeling current threshold.

Tested by: F. R. Ruppel and A. V. Blalock

Date: $\quad 1-7-92$ 
Functional Test:

Motor trips

Test Description:

Each motor circuit has a hardware trip to protect cart electronics and the cart battery from excessive currents. A Hewlett-Packard 6060A programmable load was connected to simulate high currents.

\section{Expected Result:}

Each motor circuit should trip when current exceeds 25 A. The overcurrent LED on the control pendant should light for that motor. The hardware trip should stay in effect until the motor restart button for that motor is depressed on the control pendant.

\section{Witnessed Result:}

By using the programmable load, $25 \mathrm{~A}$ was applied to the motor $\# 1$ circuit. The circuit tripped and the motor \#1 LED on the control pendant lit. The $25 \mathrm{~A}$ load was deenergized and the restart switch on the control pendant was depressed. After about a $1 \mathrm{~s}$ pause, the motor \#1 LED went off. When the cart drive button was depressed, the motor circuit energized.

Note: This functional test was not completed for motors $\$ 2-4$ because the trip function did not operate correctly. During later redesign efforts of the cart interface board, design problems were uncovered and corrected that prevented these motors from tripping.

\section{Consideration:}

When a motor restart is attempted from the control pendant, there is a short pause (1-2 s) before the motor-tripped LED goes off because of the round-trip time associated with proc'sssing the request on the facility side, transmitting the command to the cart, resetting the motor circuit on the cart, returning the new cart status to the facility, and reading the new cart status signal on the facility side.

Tested by: $\quad$ A. V. Blalock and F. R. Ruppel

Date: $\quad 1-31-92$ 
Functional Test:

Auxiliary outlet operation/telemetry

Test Description:

Operation of the cart auxiliary outlets is controlled by the control pendant. Voltage and current readings of the auxiliary outlets are available at the engineer's console display.

Expected Result:

Each auxiliary outlet should be controlled individually from the control pendant. Appropriate voltage and current readings should be read at the engineer's console display.

Witnessed Result:

Auxiliary outlet $\# 1$ was enabled from the operator's pendant. The engineer's console indicated $22.6 \mathrm{~V}$ and $0 \mathrm{~A}$ (there was no load on the outlet, hence no current)

Auxiliary outlet $\# 2$ was enabled from the operator's pendant. The engineer's console indicated $0 \mathrm{~V}$ and $30 \mathrm{~A}$. A software error was found that interchanged the voltage and current readings. This was corrected later.

Consideration:

The auxiliary outlet button must remain depressed the entire time it is desired to enable the auxiliary outlet.

Tested by: A. V. Blalock and F. R. Ruppel

Date: $\quad 1-30-92$ 
Functional Test:

Cart ID selection

Test Description:

The cart control system has the capability of communicating to one of up to four different cart electronics racks. A two-position DIP switch on the interface board of the cart electronics rack assigns the cart ID.

Expected Result:

The cart should respond to all commands received at all times regardless of cart ID with the exception of the return status command. (See Section 9 of the main report for an explanation.) The cart status should be transmitted back to the facility only when the cart ID of the electronics rack matches the cart ID byte contained in the received packet.

Witnessed Result:

From the engineer's console, the cart ID of the cart electronics being tested was enabled. Communication with the cart were maintained and telemetry of cart variables was continuously updated. When the cart ID was changed at the engineer's console, the cart variables no longer updated. However, when requested by the control pendant, the cart motor would operate in this mode.

Consideration:

If the control system does not receive new incoming data, the memory will retain old values of variables. This could be confusing; it may be necessary to zero the values when no new data is received.

Tested by: F. R. Ruppel

Date: $\quad 1-3-92$ 
Functional Test:

Motor Speed Control

Test Description:

At the engineer's console, a means exists of increasing or decreasing the speed of the cart motors.

\section{Expected Result:}

The speed of the motor should change when the increase or decrease button is depressed at the engineer's console.

\section{Witnessed Result:}

Using the engineer's console display, the cart speed window was selected. The decrease speed button was depressed several times in succession. The speed of the cart motor was noted to decrease by noting the decrease in pitch and visually noting a decrease in shaft velocity. The cart speed increase button was depressed and the motor speed was noted to increase by noting an increase in motor pitch and visually noting an increase in shaft speed.

\section{Consideration:}

The motor controller duty cycle will default to the default value after the cart computer is rebooted. Therefore, any adjustments made will be lost. The speed adjustment feature was installed mainly for testing purposes and may need to be taken out when the cart goes into operation. (The default value can be reset in software if necessary for the desired nominal cart speed.)

\section{Tested by: $\quad$ F. R. Ruppel}

Date: $\quad 1-23-92$ 
Functional Test:

Cart reboot

Test Descriptin?:

At the engineer's console there is means to reboot the cart controller in the event that proper communications with the cart fails or cart operation is deemed erratic.

Expected Result:

When the reboot button of the engineer's display is actuated the cart controller should reboot.

Witnessed Result:

The cart reboot button on the engineer's console was selected and activated. A maintenance terminal was attached to the cart computer. When the reboot button was activated, a reboot was verified by the maintenance terminal display showing the boot-up message and reactivating the count. (The cart program has a counter that counts the seconds since last booted.)

\section{Consideration:}

The cart reboot command will reboot any electronics rack that is on-line. For exarnple, if it is desired to communicate to a spare electronics rack (on the battery charger), remember thai if a cart reboot command is given, it will also reboot the operating cart electronits rack.

Tested by: M. R. Moore, R. I. Vandermolen, and F. R. Ruppel

Date: $\quad 1-20-92$ 
Functional Test:

Facility Antenna Control

Test Description:

Through the engineer's console, the engineer should have the capability to disable each facility antenna individually. This functionality is provided in the event that transmitting from more than one facility antenna causes if signal nulls. In that case one main antenna would be chosen for cart communications and the interfering antenna would be disabled.

\section{Expected Result:}

By using the engineer's console computer, all four facility antennas should be able to be disabled and enabled.

Witnessed Result:

The if syster was hardwired from the facility side to the cart side with coax, simulating the CPC antenna. When the CPC antenna was enabled at the engineer's console, the cart motor operated. When the CPC antenna was disabled, the cart motor did not operate. The procedure was repeated for the VC, tunnel, and EDR antennas.

\section{Consideration:}

If an antenna is disabled, the engineer will have to remember to reenable it later-there is no alarm of flag to remind him to do so. If it is found that it is always necessary to disable an antenna when the cart is at a certain position, the programmable logic controller could be programmed to disable and then reenable the antenna.

Tested by: M. R. Moore and F. R. Ruppel

Datc: $\quad 1-13-92$ 
Functional Test:

Calibration current

Test Description:

When the cart controller first boots up, it goes through a calibration cycle. First it checks zero values of variables that should be at zero (motor and auxiliary outlet voltages and currents). Next a 5 A calibration current is applied through the motor and auxiliary outlet current measuring circuits to allow a span calibration.

\section{Expected Result:}

Since the 5 A calibration current will be based on hand selected components, it is not likely that exactly $5 \mathrm{~A}$ will be produced from the prototype circuit with random parts selection. The most important item to be checked is whether the calibration current flows in each circuit after the cart controller is booted up.

Witnessed Result:

Calibration shunt currents for the motors were $4.68 \mathrm{~A}$ each. Calibration shunt currents for the auxiliary outlets were $4.71 \mathrm{~A}$ each. The motor currents were measured directly and the auxiliary outlet currents were measured by measuring the voltage across a $0.01 \Omega$ shunt.

\section{Consideration:}

The shunt currents were not exactly $5 \mathrm{~A}$ because hand selection of components will be necessary to achieve exactly $5 \mathrm{~A}$.

Tested by: A. V. Blalock and F. R. Ruppel

Date: $\quad 2-1-92$ 
Functional Test:

Cart fan operation

Test Description:

A fan will be used to cool cart electronics when temperatures exceed a given threshold. Since the temperature of cart electronics is monitored by the cart controller, the same temperature input can be used to actuate the fan on and off.

Expected Result:

The cart fan should be enabled at $120^{\circ} \mathrm{F}$ rising and disabled at $100^{\circ} \mathrm{F}$ falling.

Witnessed Result:

The electronics enclosure temperature sensor was heated with a heat gun. At $141^{\circ} \mathrm{F}$ the fan was enabled. When the sensor was allowed to cool, the fan was disabled when the temperature reached $119.6^{\circ} \mathrm{F}$. (Note: a software offset error was later corrected to allow the fan to come on at $120^{\circ} \mathrm{F}$ and turn off at $100^{\circ} \mathrm{F}$.)

\section{Consideration:}

There is no status signal that positively confirms the status of the fan. An indirect method to determine this is to note the electronics temperature. If it is within the fan-operation window, it is probably on.

To conserve battery charge, the fan should run as little as possible. During cart testing, it may be found that the current fan operation window can be changed some to help conserve energy, but still keep the electronics rack cool enough.

Tested by: A. V. Blalock and F. R. Ruppel

Date: $\quad 2-1-92$ 
Functional Test:

Cart position correction.

Test Description:

Software in the facility programmable logic controller calculates cart position relative to the battery charging station. (See Section 9 of the main report for a description of this algorithm.) The position indication is based on cart direction, duration of drive request, and nominal cart speed. Problems are foreseen with using this method, therefore correction points were built into the algorithm to indicate when the cart is passing a facility door threshold. When the cart limit switch actuates, it will be at a door.

Expected Result:

When the position indicates a distance within a certain tolerance of a facility door and the limit switch actuates, the position indication should correct to the distance to that door once (further actuations of the limit switch are ignored).

Witnessed Result:

North to $3 \mathrm{M}-3$ The position indication was allowed to reach -24; when the limit switch was actuated, the indication corrected to -20 .

North to 63M-008 The position indication was allowed to reach 17; when the limit switch was actuated, the indication corrected to 20.

North to 63M-001 The position indication was allowed to reach 55; when the limit switch was actuated, the indication corrected to 53 .

South to $63 \mathrm{M}-001$ The position indication was allowed to reach 62 ; when the limit switch actuated, the indication corrected to 54 .

South to 63M-008 The position indication was allowed to reach 36; when the limit switch actuated, the indication corrected to 20.

South to 3M-3 The position indication was allowed to reach -22 ; when the limit switch was actuated, the position corrected to -20 .

Note: When the limit switch was actuated away from the doors, no correction occurred.

Consideration:

Values used in this algorithm should be modified as necessary during cart testing to match the physical dimensions of the facility as close as possible.

Tested by: F. R. Ruppel

Date: $\quad 1-20-92$ 


\section{Functional Test:}

Cart antenna diversity

\section{Test Description:}

One of the feanures of the cart communications system to help prevent rf signal nulling from interfering with communications is the cart antenna diversity selection scheme. In this scheme two cart antennas are monitored for signal strength. The antenna with the greater signal strength is chosen to be used for signal reception.

Expected Result:

By changing the field around one antenna, it should be possible to cause the antenna controller to switch that antenna on and off. The cart antenna usage reading on the engineer's console should reflect the switching.

\section{Witnessed Result:}

The antenna usage was read out at the engineer's console. By walking in front of the cart antennas, antenna usage values ranged from 0 to $100 \%$.

\section{Consideration:}

The cart can switch receiving antennas only once per second. If the cart drives into a null, it will take a full second before antennas switch.

Tested by: R. I. Crutcher and F. R. Ruppel

Date: $\quad 1-23-92$ 
Functional Test:

Cart temperature telemetry

Test Description:

The cart electronics monitor the temperature of the cart battery to aid in determining the state of charge of the battery. The cart electronics also monitor the temperature of the electronics rack to provide a cold junction compensation for the battery thermocouple and to actuate the rack fan on and off with temperature.

Expected Result:

Monitor the temperature as reported on the engineer's console of the two points. Since the final version of hardware will be based on special hand-selected components, the prototype version ir not expected to be very accurate.

Witnessed Result:

The cart battery temperature, as read on the engineer's console, was $73.3^{\circ} \mathrm{F}$. The el enclosure temperature read $76.5^{\circ} \mathrm{F}$. The temperature from an independent, calibra' thermocouple readout instrument read $72^{\circ} \mathrm{F}$.

Consideration:

High accuracy temperature readings were not obtained because in the final hwill be necessary to hand select components for a good tolerance. Furtherr not designed for high accuracy, but for a rough indication of temperat battery state-of-charge indication.

Tested by: A. V. Blalock and F. R. Ruppel

Date: $\quad 1-30-92$ 
Functional Test:

Auxiliary outlet trips

Test Description:

A Hewlett-Packard $6060 \mathrm{~A}$ programmable load was placed in the auxiliary outlet circuit to allow large amounts of current to flow.

\section{Expected Result:}

When current through the auxiliary outlet circuit exceeds $25 \mathrm{~A}$, a hardware trip should become effective. The LED associated with the auxiliary outlet should flash. To reset the outlei, the operator would have to let up on the control pendant button and then depress again. If the high current condition is no longer in effect, the trip will clear.

Witnessed Result:

Each auxiliary outlet was turned on from the control pendant. Current to the circuit was raised. When a current of $25 \mathrm{~A}$ was applied to each circuit, each circuit tripped and the LED flashed on the pendant. After the trip, the reset button was pushed and the outlets reset.

\section{Consideration:}

The auxiliary outlets, when tripped, will automatically try to reset when the operator depresses the outlet button again. This logic is different than the motor reset logic, where a motor remains tripped until is requested to restart.

Tested by: $\quad$ A. V. Blalock and F. R. Ruppel

Date: $\quad 1-31-92$ 


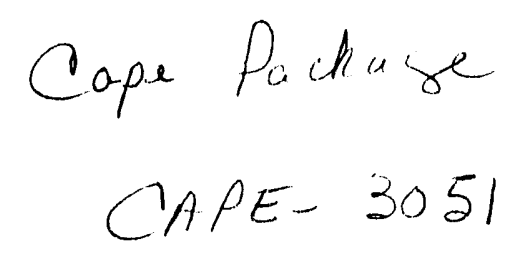

\section{APPENDIX E DRAWINGS West Valley Nuclear Services Vitrification Facility
Transfer Cart Control System Drawing List}

Rev. 4, 5/7/92

Number Sheet Rev. Title

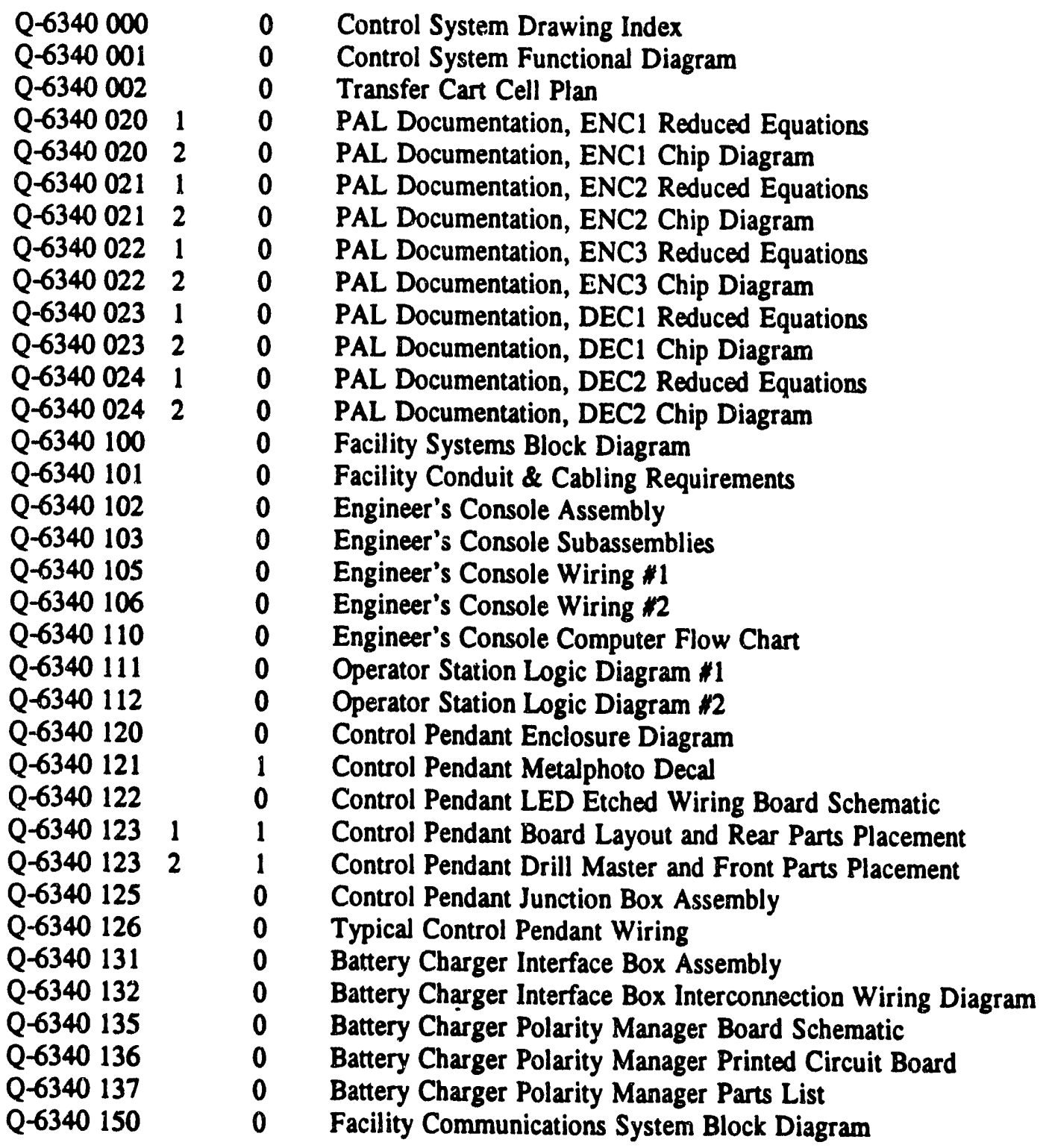


Q-6340 151

Q-6340 152

Q-6340 160

Q-6340 170

2-6340 171

Q-6340 172

Q-6340 175

Q-6340 176

Q-6340 177

Q-6340180

Q-6340 $181 \mathrm{~A}$

Q-6340 181 B

Q-6340 182

Q-6340 185

Q-6340 186

Q-6340 187

Q-6340 200

Q-6340 205

Q-6340 210

Q-6340 215

Q-6340 220

Q-6340 221

Q-6340 222

Q-6340 223

Q-6340 224

Q-6340 225

Q-6340 226

Q-6340 227

Q-6340 229

Q-6340 230

Q-6340 231

Q-6340 2312

Q-6340 232

Q-6340 233

Q-6340 235

Q-6340 236

Q-6340 240

Q-6340 244

Q-6340 245

Q-6340 246

Q-6340 247

Q-6340 250

Q-6340 260

Q-6340 261

Q-6340 262

Q-6340 265

Q-6340 266

Q-6340 267
Typical Antenna Box Assembly

Antenna Box Wiring Diagram

Facility Communications Controller Flow Chart

Facility Transmitter Schematic

Facility Transmitter Printed Circuit Board

Facility Transmitter Parts List

Facility Transmit/Receive Cell Interface Schematic

Facility Transmit/Receive Cell Interface Printed Circuit Board

Facility Transmit/Receive Board Parts List

Facility Antenna Switch Schematic

Facility Antenna Switch Printed Circuit Board \#1

Facility Antenna Switch Printed Circuit Board $\$ 2$

Facility Antenna Switch Parts List

Facility Biphase Encoder/Decoder Schematic

Facility Biphase Encoder/Decoder Printed Circuit Board

Facility Biphase Encoder/Decoder Parts List

Block Diagram

Enclosure Assembly

Cart Controller Flow Chart

Electronics Enclosure Wiring

Interface Module, Top Board, Parts Placement

Interface Module, Top Board, Layout

Interface Module, Top Board, Functional \& Battery Interface

Schematic

0 Interface Module, Top Board, Motor Control Schematic

0 Interface Module, Top Board Parts List

0 Interface Module, Middle Board, Parts Placement

0 Interface Module, Middle Board, Layout

0 Interface Module, Middle Board Schematic

0 Interface Module, Middle Board Parts List

0 Interface Module, Bottom Board Parts Placement

0 Interface Module, Bottom Board Layout, Component Side

0 Interface Module, Bottom Board Layout, Solder Side

0 Interface Module, Bottom Board Schematic

0 Interface Module, Bottom Board Parts List

0 Motor Driver Parts Placement, Layout and Schematic

0 Motor Driver Parts List

0 Cart Wiring Schedule

0 Hexfet Mounting Printed Circuit Board

0 Cart Electronics Rack Mechanical Details

0 Cart Electronics Rack Mechanical Details

0 Cart Electronics Rack Assembly

0 Cart Communications System Block Diagram

0 Facility/Cart Receiver Schematic

0 Facility/Cart Receiver Prirted Circuit Board

0 Facility/Cart Receiver Parts List

0 Cart Antenna Control Schematic

0 Cart Antenna Control Printed Circuit Board

0 Cart Antenna Control Board Parts List 
Number Sheet Rev. Title

Q-6340 270

Q-6340 271

Q-6340 272

Q-6340 290

Q-6340 291

Q-6340 292
0 Cart Transmitter Schematic

0 Cart Transmitter Printed Circuit Board

0 Cart Transmitter Parts List

0 Cart Biphase Encoder/Decoder Schematic

0 Cart Biphase Encoder/Decoder Printed Circuit Board

0 Cart Biphase Encoder/Decoder Parts List

Number Sheet Rev.Title

X3E020097A101

X3E020097A101

X3E020097A102

X3E020097A102

X3E020097A 103

X3E020097A 103

X3E020097A104

X3E020097A105

X3E020097A106

X3E020097A 106

X3E020097A107

X3E020097A108

X3E020097A108

X3E020097A109

X3E020097A110

X3E020097A111

X3E020097A111

X3E020097A112

X3E020097A113

X3E020097A114

X3E020097A115

0 Transfer Cart Control Module Interface Control Drawing

20 Transfer Cart Control Module Interface Control Drawing

10 Transfer Cart Control Module Enclosure Assembly

20 Transfer Cart Control Module Enclosure Assembly

10 Transfer Cart Control Module Enclosure Weldment

20 Transfer Cart Control Module Enclosure Weldment

0 Transfer Cart Control Module Enclosure Details

0 Transfer Cart Control Module Battery Compartment Ass'y

10 Transfer Cart Control Module Detail Sheet 1

20 Transfer Cart Control Module Detail Sheet 2

0 Transfer Cart Control Module Remote Connector Assembly

100 Transfer Cart Control Module Remote Connector Detail

20 Transfer Cart Control Module Remote Connector Detail

0 Transfer Cart Control Module Detail Sheet 2

$1 \quad 0$ Transfer Cart Control Module Detail Sheet 3

10 Transfer Cart Control Module Antenna Assembly

20 Transfer Cart Control Module Antenna Assembly

0 Transfer Cart Control Module Shielding Enclosure Weldment

0 Facility Antenna Assembly

0 Facility Antenna Details

0 Facility Antenna Details 


\section{APPENDIX F \\ OPERATIONS-MANUAL INPUT}

\section{INTRODUCTION}

The purpose of this appendix is to provide preliminary input to the operations manual for the West Valley transfer cart control system. This information is preliminary and should be updated at the completion of the fabrication and testing of the equipment. The final operation manual will be prepared by West Valley Nuclear Services, Inc., manager of the West Valley Demonstration Project.

\section{CONTROL PENDANTS}

The control pendant is depicted in drawing Q-6340-120. It is a hand-held unit through which all basic operations of the cart will be controlled. Two control pendants are used, one at the north operator's station and one at the south operator's station. The functionality of both are the same.

\subsection{OPERATION}

The cart control system operates in two basic modes: (1) battery charging and (2) cart operation. During battery charging mode, the cart is disabled completely; sending commands to the cart will have no effect, and the cart will not report status of any of its normally monitored variables. During cart operation mode, the battery cannot be charged unless it is located at the charging shoes.

The control pendant consists of switches, light-emitting diodes (LEDs), and an audible alarm. The switches and LEDs are divided among functional blocks on the front face of the pendant. The audible alarm is mounted on the top side of the pendant. The following sections describe operation of the control pendants, organized by functional blocks.

\subsubsection{Emergency Stop}

The emergency stop switch is a maintained-contact type. Depressing the switch will cause the cart to stop, disable the battery charger, and prevent any further operation of both. To undo the emergency stop condition, the red knob must be rotated clockwise. Two LEDs indicate status of emergency stops. If an emergency stop exists at this station, the top LED is lit. If an emergency stop exists at the other station, the bottom LED is lit. It is not necessary for the control pendant station to be enabled before activating an emergency stop. If the operator attempts to request an action that is inhibited by an emergency stop, the LED(s) corresponding to the emergency stop in effect will flash. 


\subsubsection{Station Select}

The control pendant station is selected by a keyswitch. The key can be inserted and removed in the $120^{\circ}$ clock position only. Inserting and rotating the key clockwise attempts to select the station for use. If the other station is not enabled, the station will be selected. Otherwise, it will not be enabled. The LEDs in this bisis indicate the status of station selection. The top LED is lit if this station is enabled and the bottom LED is lit if the other station is enabled. If the operator attempts a control pendant function other than emergency stop without first enabling the station, the station select LED will flash. If the operator attempts to select this station and the other station is already selected, the other station LED will flash. If the key is inserted and turned counterclockwise, an LED test occurs. This test consists of flashing each LED on and off regardless of its previous state. The audible alarm will be sounded continuously during this test.

\subsubsection{Doors}

Door status is given by a matrix of nine LEDs. For each of the three cell doors, status of door energized, door closed, and door open is given. If the operator attempts to drive the cart when a door is energized, the door-energized LED for that door will flash to indicate that cart drive is interlocked while a door is energized. If the operator attempts to drive the cart in close vicinity to an unopen door, the door-open LED for that door will flash, indicating that the door is not fully open.

\subsubsection{Cart Drive}

Cart drive is controlled by a three-way, return-to-center, momentary contact, rocker switch. Pressing the top of the switch requests the cart to drive north. Pressing the bottom of the switch requests the cart to drive south. If the operator is driving the cart in close vicinity to an unopen door, the LED in this block will flash, the corresponding door-open LED will flash, and the audible alarm will sound.

\subsubsection{Battery Charger}

Two momentary contact, pushbutton switches and three LEDs are provided for control and status of the battery charger. The start switch will attempt to start the battery charger. Before this operation is permitted, the cart must be located at the charging shoes. This permissive is indicated by the cart-atshoes LED. If the operator attempts to charge the batteries and the cart is not at the charging shoes, this LED will flash. When an appropriate start command has been issued the charging LED will begin to flash at a slow rate. This indicates that a valid charge command has been accepted, but that charging has not actually begun. It will take a few seconds to issue the start command to the battery charger and check to see if current is flowing. When current has begun to flow in the charging circuit, the charging LED will light steadily. If an error occurs with the charger, the charging LED will flash at a more rapid frequency than when charging is first initiated. Also, if another cart operation is attempte -but not allowed-during charging, the charging LED will flash rapidly. When the battery has been charged fully, the charging-complete LED will light. It is anticipated that the battery will remain on a float charge for long periods of time. Therefore, it is not necessary to stop charging when the charging-complete LED light first lights. When it is desired to operate the cart or disable charging for some other reason, the stop pushbutton is depressed. 
A typical charge sequence will proceed as follows:

- Operator drives cart to charge location. Cart charging plates actuate cart-at-shoes limit switch. Corresponding LED lights on control pendant.

- Operator requests battery charging by pressing the charge button on the control pendant.

- Programmable logic controller sets output to communications controller that a charge is requested and begins flashing charge light on control pendant at slow rate to indicate that a valid charge request has been made, but that charging has not commenced yet. Communications controller initiates conversation with battery charger, issues start command, and polls charger for charging data. Charger begins charging and monitors battery for current, voltage, and temperature status. Cart electronics deactivate when voltage at charging plates exceeds battery voltage. Communications controller reads status variables from battery charger and passes them to the programmable logic controller. When programmable logic controller verifies that charging current is flowing, it lights the charging light on the control pendant steadily.

- The programmable logic controller monitors charging current, voltage, and temperature. When it has determined that a full charge has been given to the batteries, it lights the charging-complete LED on the control pendant.

- When the cart is required for another transfer, the operator presses the stop charging button on the control pendant. The programmable logic controller sets an output to the communications controller to disable charging and flashes the charging light on the control pendant at a slow rate to indicate that a valid operator command has been issued, but that charging has not stopped yet. Communications controller issues command to battery charger to stop charging. Battery charger stops charging. Cart electronics are reactivated when no charging curreni exists. Programmable logic controller confirms no charge current is flowing and turns off charging LED and charging complete LED on control pendant.

- Operator presses drive button on control pendant; cart drives away from charging shoes. Cart-atshoes LED on control pendant goes off.

\subsubsection{Motors}

For each cart motor, an LED and a pushbutton are provided for status and restart control. During a motor undercurrent event, such as when a cart wheel is free wheeling, the LED will flash. For a motor overcurrent trip, the LED will light steadily. To attempt to restart the motor after an overcurrent trip, depress the restart pushbutton.

\subsubsection{Auxiliary Outlets}

For each auxiliary outlet, an LED and pushbutton are provided for status and control. To enable the auxiliary outlet, the momentary contact pushbutton is depressed and held for the duration that it is desired for the outlet to be active. When the outlet is active, the corresponding LED will light. If an overcurrent trip occurs, the LED will flash. To attempt to reset the outlet, the operator would let off of the pushbutton and then depress it again. If the operator attempts to charge the battery while an auxiliary outlet is enabled, the LED in this block will flash indicating that the battery cannot be charged until the auxiliary outlet is disabled. 


\subsection{LED LIGHTING SUMMARY}

Because only limited information is available to the operator, the control pendant has been designed to feedback as much information as possible through the LEDs. The feedback includes information to tell the operator why a denied operation is being denied by the control system, such as if the operator attempts to begin battery charging but the cart is not at the charging shoes, the can-at-shoes LED will flash. Table F.1 summarizes the meaning of the pendant LED operation.

Table F.1 Summâry of Pendant LED Operation

\begin{tabular}{|c|c|c|}
\hline LED & LIGHT STEADILY & FLASH \\
\hline $\begin{array}{l}\text { Emergency Stop } \\
\text { This Station }\end{array}$ & during emergency stop at this station & $\begin{array}{l}\text { when operation attempted during } \\
\text { emergency stop at this station }\end{array}$ \\
\hline $\begin{array}{l}\text { Emergency Stop } \\
\text { Other Station }\end{array}$ & $\begin{array}{l}\text { during emergency stop at other } \\
\text { station }\end{array}$ & $\begin{array}{l}\text { when operation attempted during } \\
\text { emergency stop at other station }\end{array}$ \\
\hline $\begin{array}{l}\text { Station Select } \\
\text { This Station }\end{array}$ & when this station enabled & $\begin{array}{l}\text { when operation attempted but this } \\
\text { station not enabled }\end{array}$ \\
\hline $\begin{array}{l}\text { Station Select } \\
\text { Other Station }\end{array}$ & when other station enabled & $\begin{array}{l}\text { when operation attempted but other } \\
\text { station enabled }\end{array}$ \\
\hline Door Energized & when door energized & $\begin{array}{l}\text { when cart drive attempted but door } \\
\text { energized }\end{array}$ \\
\hline Door Open & when door fully open & when approaching unopen door \\
\hline Door Closed & when door fully closed & \\
\hline $\begin{array}{l}\text { Driving Towards } \\
\text { Unopen Door }\end{array}$ & & when approaching unopen door \\
\hline $\begin{array}{l}\text { Battery Charger } \\
\text { Can at Shoes }\end{array}$ & when cart is at charging shoes & $\begin{array}{l}\text { when battery charge attempted but cart } \\
\text { not at shoes }\end{array}$ \\
\hline $\begin{array}{l}\text { Battery Charger } \\
\text { Charging }\end{array}$ & when batteries are charging & $\begin{array}{l}\text { slow: when valid start or stop } \\
\text { comand given but action not taken yet } \\
\text { fast: when cart operation requested but } \\
\text { batteries are charging }\end{array}$ \\
\hline $\begin{array}{l}\text { Battery Charger } \\
\text { Complete }\end{array}$ & when batteries fully charged & \\
\hline $\begin{array}{l}\text { Motor } \\
\text { Overcurrent } \\
\text { Undercument }\end{array}$ & during motor overcurrent trip & when motor free-wheeling \\
\hline Auxiliary Outlet & when outlet enabled & when outlet tripped \\
\hline
\end{tabular}




\section{ENGINEER'S CONSOLE}

The engineer's console is depicted in drawing Q-6340-102. It houses the workstation through which the engineer communicates to the cart control system. It also houses major facility-side control system components such as the Allen-Bradley programmable logic controller (PLC), the Prolog communications controller, and ancillary equipment.

\subsection{OPERATION}

Intouch, a man-machine interface will be running on the engineer's console computer. Through Intouch, the engineer can monitor and record cart variables and pendant activity and can modify limited system options. The workstation is the primary engineer's interface. However, a keyswitch on the front of the console is also provided for cart orientation. Following are descriptions of the operation of the cart orientation keyswitch and the Intouch man-machine interface.

\subsubsection{Cart Orientation Keyswitch}

A keyswitch is mounted on the front of the engineer's console to serve the purpose of changing the cart orientation status to the PLC. The same switch indicates that battery charging polarity must be reversed. This switch should be changed when the cart has been reversed on the tracks. It is very important that the engineer use this switch, as it is the control system's only way of determining which direction to drive the cart and what polarity to apply to the battery charging shoes.

\subsubsection{Alarm and Event Summary}

A window on the engineer's interiace is dedicated to a summary of active alarms and events. This window can be used to indicate key items to the engineer such as cart ID selected, whether any facility antennas are disabled, what control pendant is selected, whether the battery charger or cart is active, which way the cart is oriented, etc. Alarms will also show such as motor over- and undercurrents, low battery voltage, approaching unopen door, etc. The alarms and events shown in this window are temporary-when the condition is no longer in effect, the line will disappear. However, a log of the alarm or event is saved to disk so that there is always a record of it.

\subsubsection{Cart and Door Position}

A pictorial representation of cart and door position is given in this window. The cart is shown on tracks, where north is left. The correct cart orientation should appear, and switching the cart orientation keyswitch should switch orientation of the cart on the screen. The position of the cart, as determined by the cart position algorithm in the PLC, is also given. Position of each door is shown. For doors 63M001 and $3 \mathrm{M}-3$, the door is shown by a solid rectangle covering the tracks when the door-closed limit switch is active. When the door-open limit switch is active, the rectangle slides off the tracks. When neither limit switch is active, the door is shown in a middle position. The same type logic applies to door 63M-008 except that two swinging doors are shown instead. When the PLC's door alarm is in effect, the door that the cart is approaching will flash in red. 


\subsubsection{Cart Status}

Status of cart variables in numeric form is presented in this window. Values of motor and battery voltages and currents are listed as well as battery and electronics enclosure temperature.

\subsubsection{Facility Antenna Control}

To enable or disable facility antennas, the engineer calls up the facility antenna control window. This window has four buttons, one for each facility antenna. The text on each button shows the option available for each antenna, either DISABLE or ENABLE. To command an antenna on or off, the engineer would select the button with the tab key and depress the return key. The text on the button will toggle, always showing what the new option is. FOY example, if working with the VC antenna initially enabled, if the button is hit, the text will toggle from DISABLE VC to ENABLE VC.

\subsubsection{Cart ID Selection}

A total of four cart IDs are available to tag the main cart system, spare cart system, and spare electronics racks. By calling up the Cart ID Selection window, the engineer can select to which cart ID the facility communications system should direct its messages. Four buttons are presented with text corresponding to available options. If a given ID is currently active, selecting that button will have no effact. But selecting a new cart ID will change the message of the new cart ID button as well as the old cart ID button. For example, if cart ID \#1 is currently active, the first button will read $\# I A C T T V E$, the second through fourth buttons will read ACTTVATE \#2, ACTTVATE \#3, and ACTTVATE \#4. If the second button is selected with the tab key, and the return key is depressed, the text on buttons one through four will change to ACTIVATE \#1, \#2 ACTIVE, ACTIVATE \#3, and ACTIVATE \#4.

\subsubsection{Real-Time Trends}

Real-time trends of all cart variables are available. In many cases the trends are grouped into like variables, but the maximum number of plots per trend is four. For example all motor currents are shown on the same trend and all motor voltages are shown on another trend.

\subsubsection{Historical Trends}

It is possible to call up historical data that has been saved to disk by using the historical trend function.

\subsection{MAINTENANCE}

It is possible to enter maintenance modes on other system components by using the engineer's console workstation. The maintenance modes are described in the following sections.

\subsubsection{Battery Charger}

By using a terminal emulation program, the engineer can communicate directly with the battery charger, but first a switch box located in the rear of the engineer's console must be switched to connect the battery charger to the workstation computer rather than the Prolog communications controller. The battery charger can be reprogrammed, charger status can be displayed, and a past history of charging variables 
can be requested. If necessary the charger can be started and stopped from here also, although this task is normally done by the Allen-Bradley PLC through the Prolog communications controller by request from the control pendant.

\subsubsection{Prolog Communications Controller}

The terminal emulation program can also be used to communicate to the serial port of the Prolog communications controller. The controller has a maintenance mode that can be activated by doing this. Status of the controller is provided. It is also possible to download new programs to the controller in this mode.

\subsubsection{Allen-Bradley PLC}

The engineer's workstation can also be used to tie directly into the Allen-Bradley PLC. In this mod- the ladder logic of the PLC can be displayed online and modified if necessary. Inputs and outpur. be forced on and off. In addition to online modifications, a new program can be downloaded to "'

\section{CART CONTROLLER}

The cart controller is a Winsystems single board computer. During normal communicate with the facility and its operation will be directed by ei॰ pendants or the engineer at the engineer's console. However, the $r$ mode that enables interrogating operation of the cart controller in

\subsection{MAINTENANCE MODE}

To use the maintenance mode of the cart controller, a terminal 1. serial port. When connected in this manner, the cart controller $e_{\text {. }}$ mode local cart functions can be checked such as motor control, fan c Status information of all cart variables can be shown as well as sh. verification of communications packets can be done also. 
ORNL/TM-12263

\section{INTERNAL DISTRIBUTION}

1. G. A. Armstrong

2. S. M. Babcock

3. J. F. Birdwell

4-6. E. C. Bradley

7. T. W. Burgess

8. B. L. Burks

9. P. L. Butler

10. D. L. Canter

11. J. B. Chesser

12. H. M. Costello

13. R. I. Crutcher

14. F. W. DePiero

15. J. V. Draper

16. J. E. Dunn, Jr.

17. B. G. Eads

18. M. H. Ehinger

19. R. L. Glassell

20. Nk. J. Haire

21. D. C. Haley

22. J. W. Halliwell

23. W. R. Hamel

24. J. H. Hannah

25. J. E. Hardy

26. J. N. Herndon
27. R. A. Hess

28. M. S. Hileman

29. J. F. Jansen

30. R. T. Jubin

31. S. M. Killough

32. R. L. Kress

33. C. T. Kring

34. D. W. Kwon

35. B. E. Lewis

36. P. D. Lloyd

37. E. Madden

38. B. L. Malone

39. S. March-Leuba

40. J. A. McEvers

41. D. W. McDonald

42-44. S. A. Meacham

45. C. F. Metz

46. M. R. Moore

47. M. W. Noakes

48. R. N. Nodine

49. T. E. Noell

50. R. E. Norman

51. C. H. Nowlin

52. K. E. Plummer
53. T. L. Ray

54. B. S. Richardson

55. J. C. Rowe

56-58. F. R. Ruppel

59. J. H. Saling

60. S. L. Schrock

61. B. H. Singletary

62. C. O. Slater

63. G. E. Smith

64. B. B. Spencer

65. J. W. Stapleton

66. D. H. Thompson

67. K. U. Vandergriff

68. R. I. Vandermolen

69. K. L. Walker

70. B. S. Weil

71. K. Wilson

72. H. R. Yook

73-74. Laboratory Records

75. Laboratory Records-RC

76. RPSD Publications Office

77. ORNL Patent Section

78. Central Research Library

79. Document Reference Section

\section{EXTERNAL DISTRIBUTION}

80. Clinton Bastin, Manager, LMR Reprocessing Projects, Division of Fuels and Reprocessing, Office of Facilities, Fuel Cycle, and Test Programs, NE-471, Department of Energy, Washington, DC 20545.

81. S. R. Martin, Jr., Acting Program Manager, Fusion and Nuclear Technology Branch, Energy Programs Division, Department of Energy, X-10 Site, P.O. Box 2008, Oak Ridge, Tennessee 37831-6269.

82. R. A. Meigs, West Valley Nuclear Services Inc., P.O. Box 191, MS-59, Rock Springs Road, West Valley, New York 14171-0191.

83. WVNS Technical Library, West Valley Nuclear Services Inc., P.O. Box 191, MS-45, Rock Springs Road, West Valley, New York 14171-0191.

84. Office of Assistant Manager for Energy Research and Development, Oak Ridge Operations Office, Department of Energy, P.O. Box 2008, Oak Ridge, Tennessee 37831-6269.

85-86. Office of Scientific and Technical Information, P.O. Box 62, Oak Ridge, Tennessee 37831. 

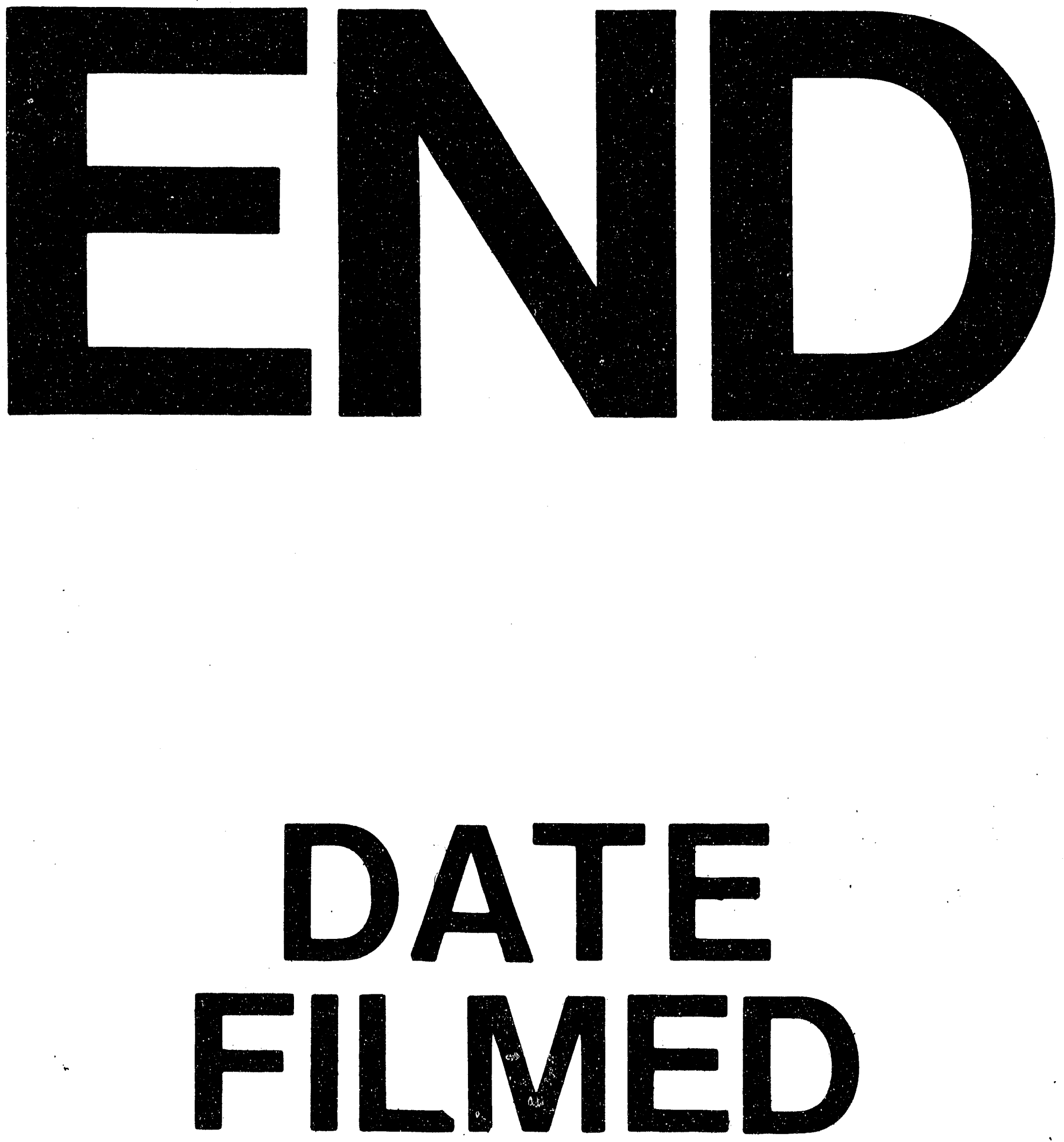

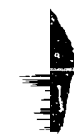

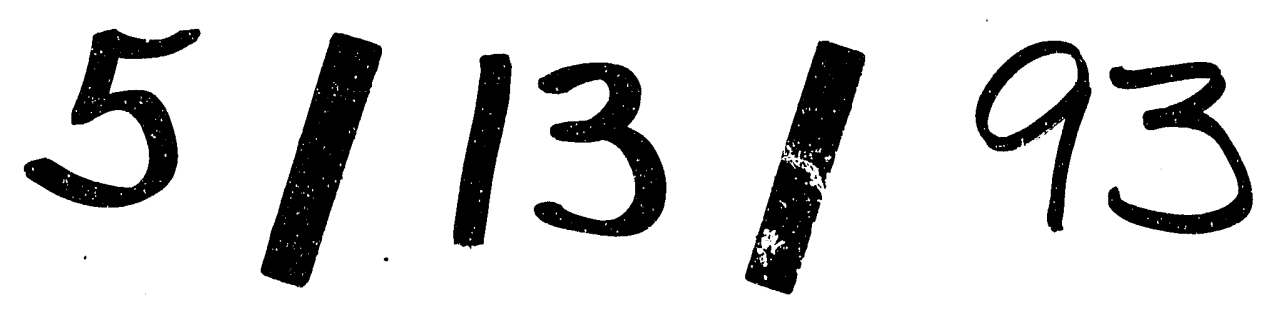


